Merci d'utiliser le titre suivant lorsque vous citez ce document :

Moreddu, C. (2011-08-10), « Distribution du soutien et des revenus dans l'agriculture », Éditions OCDE, Paris.

http://dx.doi.org/10.1787/5kg6v2hwlpxw-fr

\title{
Distribution du soutien et des revenus dans l'agriculture
}

\author{
Catherine Moreddu
}

La version originale de ce document a été publiée comme suit :

Moreddu, C. (2011-05-01), "Distribution of Support and Income in Agriculture", OECD Food, Agriculture and Fisheries Papers, No. 46, OECD Publishing, Paris.

http://dx.doi.org/10.1787/5kgch21wkmbx-en 


\title{
DOCUMENTS DE TRAVAIL DE L'OCDE SUR L'ALIMENTATION, L'AGRICULTURE ET LES PÊCHERIES
}

La série des documents de travail de l'OCDE permet de mettre à la disposition d'un public plus large une sélection d'études effectuées soit par le personnel de l'OCDE, soit par des consultants externes. Ces documents sont disponibles, en général, uniquement dans leur langue d'origine (anglais ou français).

Ce document a été déclassifié par le Groupe de travail sur les politiques et marchés de l'agriculture du Comité OCDE pour l'agriculture.

Tout commentaire sur cette série sera apprécié et devra être adressé à tad.contact@oecd.org.

\author{
LES DOCUMENTS DE TRAVAIL DE L'OCDE SUR \\ L'ALIMENTATION, L'AGRICULTURE ET LES PÊCHERIES \\ sont publiés sur www.oecd.org/agriculture
}

(C) OECD 2011

Toute demande pour usage public ou commercial de ce rapport doit être addressee à OECD Publishing, rights@ oecd.org or by fax 33145249930. 


\title{
Résumé
}

\section{DISTRIBUTION DU SOUTIEN ET DES REVENUS DANS L'AGRICULTURE}

\author{
Catherine Moreddu \\ Économiste principale, OCDE
}

Au Canada, aux États-Unis et dans les États membres de l'Union européenne, la production et le soutien agricoles sont extrêmement concentrés dans les plus grandes exploitations, dont les niveaux de revenu sont plus élevés que la moyenne de l'ensemble des exploitations. Cependant, les plus petites exploitations dépendent davantage du soutien, en particulier des paiements, qui contribuent dans une proportion plus élevée à leur chiffre d'affaire. Comme les paiements versés aux agriculteurs sont répartis de manière plus égale que la production, le soutien public améliore légèrement la distribution du revenu selon la taille et type de l'exploitation. Cette étude, réalisée dans le cadre du réseau de l'OCDE pour l'analyse au niveau de l'exploitation, conclut que pour améliorer l'efficience et l'équité des politiques agricoles, il conviendra de mieux cibler le soutien du revenu et donc de disposer d'une meilleure information sur la situation de la population agricole en matière de revenu et de richesse.

Mots clés : politique agricole, soutien agricole, distribution du soutien, distribution du revenu, richesse agricole, équité, ciblage.

Classification JEL : D31, Q12, Q18.

\section{Remerciements}

Ce rapport a été établi dans le cadre d'un projet entrepris par le Réseau de l'OCDE sur l'analyse au niveau de l'exploitation. Le réseau a examiné la définition du projet et établi les termes du mandat. Les membres du réseau qui ont choisi de participer au projet ont fourni des tableaux normalisés à partir d'un modèle préparé par un sous-groupe de participants. Catherine Moreddu, du Secrétariat de l'OCDE, a rédigé un rapport analysant les informations reçues. Florence Bossard a apporté son soutien sur les questions statistiques. Les commentaires des membres du réseau ont permis d'améliorer le rapport. 


\section{Table des matières}

Résumé

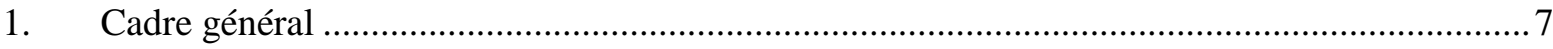

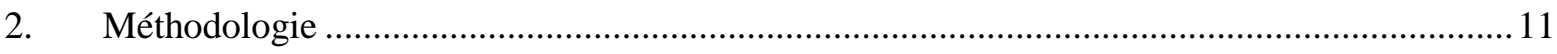

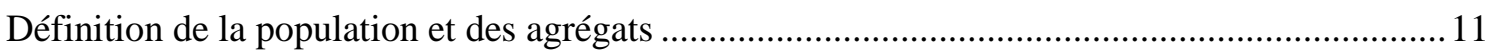

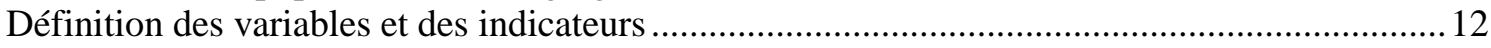

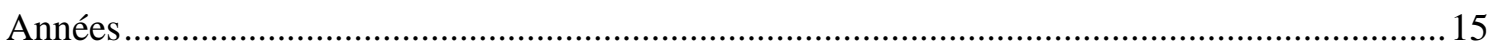

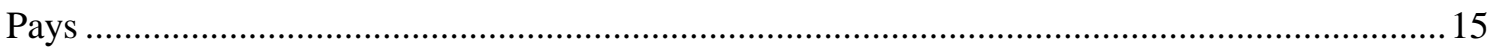

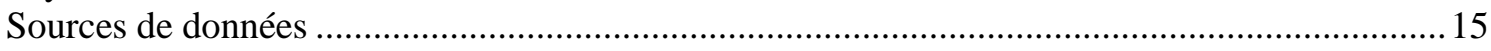

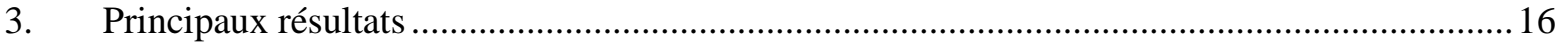

Distribution du soutien, des revenus et des actifs par taille d'exploitation ...................................16

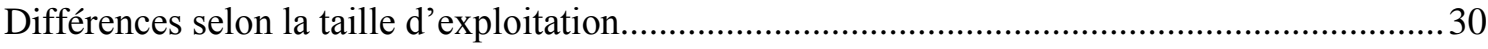

Distribution du soutien et des revenus par type d'exploitations...................................................... 32

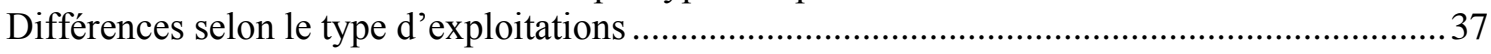

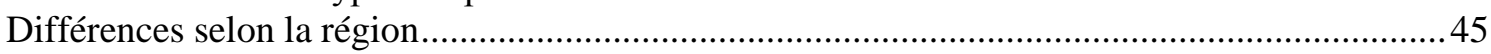

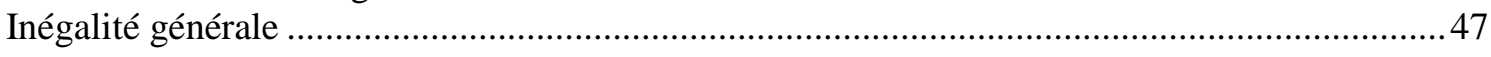

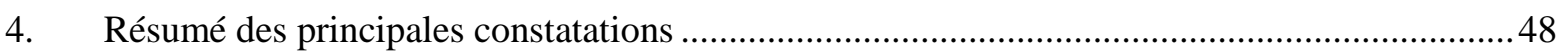

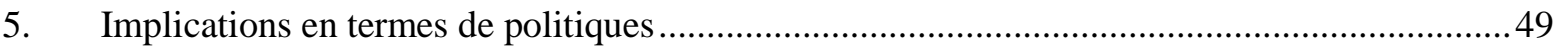

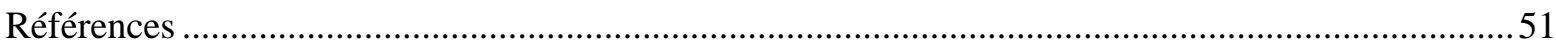

Annexe A. Liste des tableaux fournis par les participants............................................................52

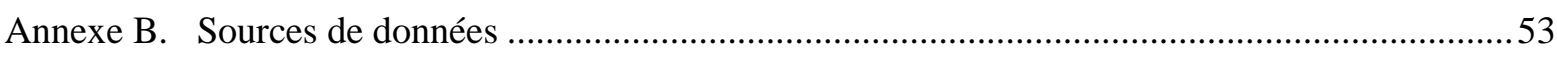

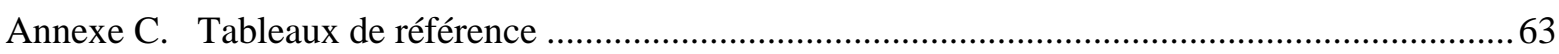

\section{Tableaux}

Tableau 1.1. Participants au projet relatif à la distribution du soutien et des revenus ........................ 10

Tableau annexe C1. Part des $25 \%$ d'exploitations les plus grandes dans certains pays de l'OCDE, par composante du revenu, 2004, 2006 et 2007.

Tableau annexe C2. Part des $25 \%$ d'exploitations les plus petites dans certains pays de l'OCDE, par composante du revenu, 2004, 2006 et 2007

Tableau annexe C3. Ratio de la moyenne des exploitations regroupées par taille d'exploitation, sur la moyenne de l'ensemble des exploitations, certains pays de l'OCDE, 2004, 2006, 2007

Tableau annexe $\mathrm{C} 4$. Part du soutien dans les recettes des exploitations et dans le revenu agricole, par taille d'exploitation, certains pays de l'OCDE, 2004, 2006 et 2007. .76

Tableau annexe C5. Ratio de la moyenne des exploitations regroupées par type d'exploitations, sur la moyenne de l'ensemble des exploitations, 2004, 2006, 2007. 
Tableau annexe C6. Part du soutien dans les recettes des exploitations et dans le revenu agricole, 2004, 2006, 2007

Tableau annexe C7. Ratio de la moyenne des exploitations regroupées par type de régions, sur la moyenne de l'ensemble des exploitations, certains pays de l'OCDE, 2004, 2006, 2007 .....98

Tableau annexe C8. Part du soutien dans les recettes des exploitations et dans le revenu agricole, par type de régions, certains pays de l'OCDE, 2004, 2006 et 2007

Tableau annexe C9. Indices de Gini dans certains pays de l'OCDE, par composante du revenu, 2004, 2006 et 2007

\section{Graphiques}

Graphique 3.1. Part des $25 \%$ d'exploitations les plus grandes dans certains pays de l'OCDE, par composante du revenu, 2007

Graphique 3.2. Part des $25 \%$ d'exploitations les plus petites dans certains pays de l'OCDE, par composante du revenu, 2007.

Graphique 3.3. Courbes comparant la distribution des composantes du soutien et du revenu, 2007.

Graphique 3.4. Courbes comparant la distribution des composantes du soutien, 2007 ....................24

Graphique 3.5. Courbes comparant la distribution des revenus et des actifs, 2007 ........................27

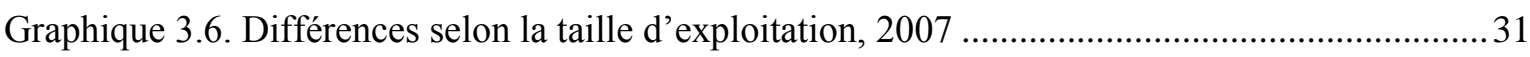

Graphique 3.7. Part du soutien dans les recettes agricoles brutes, par taille d'exploitation, 2007 ...32

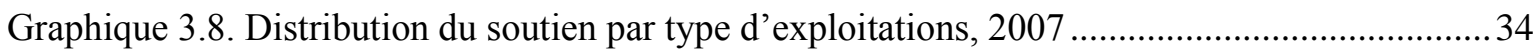

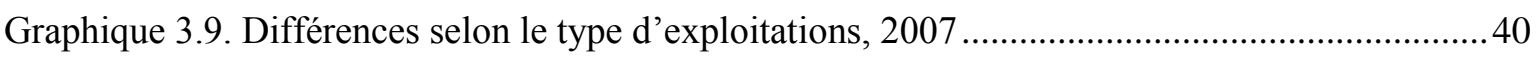

Graphique 3.10. Part du soutien dans les recettes agricoles brutes, par type d'exploitations, 2007

Graphique 3.11. Différences selon le type de régions, 2007 ..........................................................46

Graphique 3.12. Part du soutien dans les recettes agricoles brutes, par type de régions, 2007 ........46

Graphique 3.13. Comparaison des indices de Gini par pays et par composante des recettes, 2007.

Graphique 3.14. Comparaison des indices de Gini par pays de l'Union européenne et par type de paiements, 2007

\section{Encadrés}

Encadré 1.1. Réseau de l'OCDE sur l'analyse au niveau de l'exploitation: déclaration

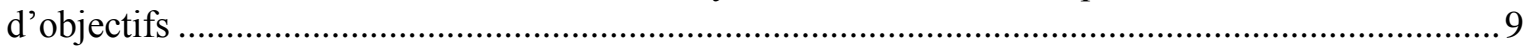

Encadré 2.1. Liste et définition des variables des tableaux standard .............................................. 13

Encadré 2.2. Indicateurs et terminologie utilisée pour décrire la distribution des variables.............15 


\section{Résumé}

Les évaluations de politiques doivent déterminer avec précision qui bénéficie du soutien, si ces bénéficiaires sont ceux visés par les politiques évaluées et si celles-ci sont efficaces et à quel prix. L'évaluation doit tenir compte de l'ensemble des coûts et avantages, sans oublier les effets secondaires et les coûts de mise en œuvre. Ce rapport s'efforce d'apporter sa contribution à l'évaluation des performances des politiques poursuivant des objectifs de revenu, et s'inscrit dans les efforts consentis de longue date par l'OCDE pour améliorer les données disponibles dans ce domaine. Il fournit des informations de base sur la distribution du soutien et des revenus par taille d'exploitation, par type d'exploitations et par région dans un certain nombre de pays membres de l'Organisation. Considérant plusieurs types de paiements et de soutien des prix du marché, il compare, pour différentes catégories d'exploitations, les niveaux moyens de soutien et leur part dans les recettes agricoles. Une certaine harmonisation des exploitations couvertes et des catégories a été réalisée à l'aide de données d'enquêtes, autorisant dans une certaine mesure les comparaisons entre pays. Treize pays de l'OCDE et la Commission européenne ont fourni les données exploitées dans ce rapport. Malgré la diversité de ces pays et régions, il est possible de tirer quelques conclusions générales sur les caractéristiques des principaux bénéficiaires du soutien et sur leur situation de revenu.

Dans la plupart des pays examinés, la production et le soutien agricoles sont fortement concentrés sur les plus grandes exploitations (taille mesurée par la valeur de la production agricole brute): les $25 \%$ d'exploitations les plus grandes enregistrent 45 à $85 \%$ de la production agricole brute et reçoivent 35 à $75 \%$ du soutien total. Elles représentent également 50 à $75 \%$ du revenu agricole total, et leur revenu individuel moyen dépasse largement celui de l'ensemble des exploitations. En dépit de sa répartition inégale et du fait qu'il profite principalement aux plus grandes exploitations, lesquelles disposent d'un revenu agricole moyen plus élevé, le soutien est légèrement moins inégalement réparti que la production agricole brute. Ainsi, toutes choses égales par ailleurs, il améliore légèrement l'égalité de la distribution des recettes brutes et des revenus par taille d'exploitation. Dans la mesure où le soutien des prix du marché est étroitement lié à la production des produits de base, sa distribution est encore plus inégale que celle des paiements. Les plus petites exploitations étant généralement plus dépendantes de sources de revenu non agricoles que les plus grandes, la prise en compte de ces sources réduit les inégalités de revenu par taille d'exploitation. Les capitaux propres sont également concentrés sur les exploitations les plus importantes, quoique dans une moindre mesure par rapport à la production, au soutien ou aux revenus, tandis que la distribution des dettes agricoles est proche de celle des revenus ou légèrement plus inégale, indiquant que les petites exploitations sont proportionnellement moins endettées que les plus grandes. Si les entreprises de moindre taille reçoivent une plus faible proportion du soutien total, celui-ci, et en particulier les paiements, représente une plus grande part de leurs recettes brutes.

Dans de nombreux pays, le soutien se concentre sur quelques types d'exploitations (production végétale, élevage laitier et élevage bovin, principalement) et il existe de 
grandes différences de concentration entre le soutien des prix du marché et le total des paiements. Cet état de fait traduit des variations du taux et de la composition du soutien selon les produits de base, mais aussi la part de certains types d'exploitations dans la population agricole totale. De fait, les différences observées dans le niveau moyen de soutien et de revenu sont plus importantes par type que par taille d'exploitation. Dans de nombreux cas, toutefois, il semble que le soutien réduise les écarts de revenu entre types d'exploitations. Ce constat est particulièrement clair dans les États membres de l'Union européenne où les exploitations avicoles, fruitières et maraîchères à haut revenu reçoivent peu de paiements, mais où les élevages à faible revenu de bovins et d'ovins à l'herbe sont fortement aidés, en particulier dans les zones les plus défavorisées. La corrélation entre certaines formes d'aide et les conditions du marché des produits de base (soutien des prix du marché et paiements contracycliques au Canada et aux États-Unis) accentue les variations d'une année sur l'autre du soutien entre types d'exploitations par rapport à celles observées selon la taille d'exploitation et la région. L'analyse par type de régions fait apparaître des écarts de revenu généralement plus faibles, que le soutien semble réduire. Les indices de Gini, qui mesurent les inégalités entre exploitations individuelles en dehors de toute classification, confirment que le soutien n'a qu'un faible effet redistributif sur le revenu.

La distribution du soutien dépend des caractéristiques des politiques, en particulier du lien avec la production de produits de base et du degré de ciblage, qui affectent également l'efficacité de transfert du soutien en termes de revenu, c'est-à-dire la partie du soutien transmise aux producteurs agricoles sous forme de revenu supplémentaire. Cette partie est différente pour chaque mesure, selon la part du soutien capturée par les fournisseurs d'intrants et la filière alimentaire en aval. La question de l'efficacité de transfert du soutien en termes de revenu n'est toutefois pas abordée dans ce rapport. La distribution du soutien dépend aussi fortement des caractéristiques structurelles de la population agricole, et ce d'autant plus lorsque le soutien n'est pas ciblé avec précision. L'effet redistributif variera donc par région ou par pays pour une politique donnée. Ces considérations doivent être prises en compte dans l'élaboration de politiques que l'on souhaite efficaces et efficientes, et dans l'examen de leur équité. L'amélioration de l'efficience et de l'équité des politiques exigerait un meilleur ciblage du soutien des revenus, et donc une information plus fouillée sur les revenus et la situation patrimoniale de la population agricole. 


\section{Cadre général}

Dans bon nombre de pays de l'OCDE, les politiques agricoles donnent lieu à des transferts importants des contribuables et des consommateurs aux agriculteurs. En 2009, ces transferts se sont élevés à 250 milliards USD, soit $22 \%$ des recettes agricoles de la zone OCDE. Certaines politiques agricoles comportent des objectifs redistributifs explicites ou implicites, et visent notamment à améliorer le revenu des populations agricoles ou à réduire les coûts de production des exploitations. D'autres, en revanche, rémunèrent les services environnementaux, ce qui signifie que les transferts sont liés à la valeur des services fournis ou au coût de la prestation.

Quel que soit l'objectif recherché, la question de la distribution du soutien entre agriculteurs suscite un intérêt croissant dans un grand nombre de pays de l'OCDE. À mesure que les paiements budgétaires remplacent le soutien des prix du marché, les mécanismes d'aide deviennent de plus en plus visibles et attirent donc l'attention du public. En conséquence, un nombre croissant de pays publient aujourd'hui des statistiques sur la distribution des paiements budgétaires destinés aux agriculteurs. On dispose de peu d'informations sur la distribution du soutien des prix du marché $(\mathrm{SPM})^{1}$, parce que la partie de ce soutien qui correspond à des dépenses budgétaires ne donne pas lieu à des versements aux producteurs individuels et que l'autre partie est payée par les consommateurs.

Quand le soutien est associé au volume de production, la distribution est nécessairement très inégale, et ce sont les grandes exploitations qui en bénéficient le plus (OCDE 1999, 2003). Comme le changement structurel conduit à une plus grande spécialisation de la production et à une concentration accrue dans les exploitations agricoles, il accroit les inégalités dans la distribution du soutien lié à la production. Cela étant, la réforme des politiques a modifié le mode d'octroi du soutien, et le lien avec la production effective de produits précis est souvent moins marqué dans les mesures prises aujourd'hui. Doit-on en conclure que les paiements «découplés» sont répartis de manière plus équitable ? Il ne faut pas s'attendre à des changements spectaculaires car les niveaux de paiement découlent souvent de la structure de production antérieure. Cependant, le fait de rompre le lien entre le soutien et les modèles précédents d'aide à la production de produits de base permet de cibler des objectifs spécifiques.

L'intérêt que suscite la distribution du soutien peut être nourri uniquement par la recherche de l'équité ${ }^{2}$ ou, plus précisément, par l'effet de ce soutien sur la distribution des revenus des ménages agricoles. Le soutien ou la stabilisation du revenu est un objectif majeur des politiques agricoles dans de nombreux pays de l'OCDE, et il est utile d'évaluer les politiques au regard de cet objectif, mais la distribution du soutien peut aussi avoir des répercussions sur l'efficience économique.

L'OCDE a travaillé sur ces questions au début des années 2000. Pour déterminer la performance des politiques en référence à des objectifs de revenu, une étude réalisée en 2003 (OCDE, 2003) s'est intéressée à la distribution du soutien selon la taille des exploitations agricoles dans certains pays de l'OCDE, en utilisant des quartiles établis à

1. Le soutien des prix du marché comprend les transferts venant des contribuables et des consommateurs, qui créent un écart entre les prix du marché intérieur et les prix à la frontière. Il est calculé par l'OCDE pour certains produits et entre dans le calcul de l'Estimation du soutien aux producteurs (ESP) de l'Organisation (encadré 2).

2. L'équité a été reconnue par les ministres de l'OCDE comme un critère opérationnel d'évaluation des politiques agricoles (OCDE, 1998). 
partir du chiffre d'affaires brut ${ }^{3}$. L'étude comparait la distribution du soutien (y compris le soutien des prix du marché) avec celle des revenus, afin d'évaluer les effets redistributifs sur le revenu agricole du soutien des prix du marché et des paiements budgétaires. L'examen des besoins et déficits d'information auquel cette étude a donné lieu a fait apparaitre que les différences relevées dans les définitions de termes « exploitation agricole », «ménage agricole » ou « revenu » rendaient très difficiles les comparaisons entre pays.

Cette étude et d'autres projets ont permis de se rendre compte qu'il importait de disposer de données microéconomiques pour améliorer l'analyse des politiques. Différentes approches ont été suivies lors de travaux précédents. Elles ont principalement consisté soit à adresser des questionnaires à des responsables de bases de données d'enquêtes agricoles travaillant pour des instituts publics afin de recueillir des renseignements sur des groupes personnalisés (quartiles fondés sur les recettes agricoles), soit à faire appel à des consultants ayant accès à des données microéconomiques afin de mener une analyse précise.

Effectuer une analyse microéconomique en interne à l'OCDE est difficile et coûteux pour les raisons suivantes :

- L'accès aux données microéconomiques n'est pas facile, tant sur le plan institutionnel que technique ${ }^{4}$, en particulier pour une organisation internationale qui cherche en principe à analyser plusieurs pays simultanément, de préférence sur une base comparable. La résonance politique (pour les données sur les revenus, par exemple) et le respect des règles et réglementations en matière de confidentialité et de respect de la vie privée limitent l'accès aux données individuelles et la possibilité de relier des sources d'information différentes.

- Procéder à des analyses régulières au niveau microéconomique exigerait d'importantes ressources en temps et en argent ainsi que des connaissances spécialisées, qui ne sont pas toujours disponibles.

Pour des raisons juridiques, mais aussi parce qu'ils possèdent le savoir-faire technique nécessaire et une connaissance approfondie de leurs pays respectifs, les instituts nationaux de recherche sont mieux placés pour accéder aux données microéconomiques et les exploiter. Ainsi, le programme de travail et budget (PTB) 2007-08 du Comité de l'agriculture comportait un projet de création d'un réseau d'instituts officiels de statistiques et de recherche, afin d'aider l'OCDE dans ses efforts d'analyse des politiques au moyen de données microéconomiques et d'informations infranationales (encadré 1.1). La contribution de ce réseau au travail du Comité de l'agriculture a également été inscrite au PTB 2009-10 (Domaine de résultats 3.2.1: Résultats intermédiaires 1.5: Suivi et évaluation des politiques agricoles des pays de l'OCDE : Collaboration extérieure).

3. Australie, Canada, Corée, Danemark, États-Unis, Finlande, Japon, Pays-Bas, Suisse et Union européenne.

4. La faisabilité de l'accès à des données microéconomiques a été évaluée par la Direction des statistiques de l'OCDE. Une conférence s'est tenue au Luxembourg les 26 et 27 octobre 2006. Les documents et les exposés présentés lors de cette conférence peuvent être consultés à l'adresse suivante : https://www.oecd.org/document/27/0,2340,fr_2649_201185_37502683_1_1_1_1,00.html. 


\section{Encadré 1.1. Réseau de l'OCDE sur l'analyse au niveau de l'exploitation : déclaration d'objectifs}

Le réseau a été créé en 2008 sous les auspices de l'OCDE. II compte dans ses rangs des experts travaillant pour des institutions à caractère public et autres instituts de recherche en économie agricole, qui participent à la collecte ou à l'analyse de données microéconomiques. L'adhésion est volontaire et l'objectif est d'obtenir une couverture représentative des pays de l'OCDE. Le Secrétariat de l'OCDE est chargé de convoquer les membres du réseau et les délégués aux réunions de l'OCDE, et d'assurer la liaison entre toutes les personnes concernées.

Les membres du réseau et l'OCDE ont comme objectif commun d'améliorer la qualité et l'utilité de l'analyse des politiques appliquée au secteur agricole par l'emploi de données microéconomiques, et reconnaissent de ce fait le besoin croissant de disposer de données fiables de cette nature et des outils d'analyse associés pour faciliter l'élaboration des politiques.

Le principal objectif du réseau est donc d'appuyer l'analyse de politiques à laquelle l'OCDE s'emploie, au moyen de données microéconomiques et d'informations infranationales. Ce dispositif devrait contribuer aux projets de l'Organisation en fournissant une analyse microéconomique portant sur plusieurs pays et réalisée sur des bases cohérentes. Dans les projets inscrits au programme de travail du Comité de l'agriculture de l'OCDE, il est prévu que le réseau recense les questions susceptibles de bénéficier d'une approche microéconomique, détermine les sources de données et propose des méthodes innovantes et adaptées.

L'un des autres objectifs du réseau est d'échanger des expériences et de démontrer de quelle manière l'étude microéconomique peut être utilisée aux fins d'analyse des politiques. Pour y parvenir, il sera nécessaire de communiquer des analyses pertinentes et de s'interroger sur les données utilisées et la façon dont elles sont étudiées. Dans le cadre de cet objectif, il appartient également au réseau d'attirer l'attention des délégués sur les nouvelles questions de fond auxquelles les approches microéconomiques pourraient bénéficier, de façon à alimenter la réflexion sur le programme de travail à plus long terme.

Le réseau est opérationnel depuis 2008. À sa première réunion, les participants ont décidé de s'intéresser à la distribution du soutien. Le consensus s'est fait sur l'idée de commencer par une étude relativement simple afin de tester le fonctionnement du réseau. La présentation de cette étude a été débattue lors de la deuxième réunion du réseau, les 29 et 30 septembre 2008, et il a été décidé que le projet devrait répondre à deux questions :

- Comment le soutien est-il distribué entre exploitants selon les caractéristiques retenues (exemples : type d'exploitations/spécialisation, région, taille économique ou revenu) ?

- Comment le soutien affecte-t-il le niveau et la distribution des recettes brutes et des revenus?

- Comment les composantes du revenu et du patrimoine sont-elles distribuées ?

Un mandat clair a été arrêté à l'occasion de la troisième réunion qui s'est tenue le 26 février 2009. Des tableaux types ont ensuite été élaborés par un groupe de participants et transmis à toutes les personnes concernées. Ces tableaux ont été révisés depuis afin d'y ajouter des variables. Le présent rapport repose sur les données communiquées par les pays ou régions mentionnés au tableau 1.1.

Comparé aux précédents travaux de l'OCDE, ce projet actualise l'analyse et améliore la cohérence entre les pays, car les définitions ont été harmonisées dans la mesure du possible (voir la section Méthodologie). Il fournit également des informations supplémentaires harmonisées, par type d'exploitations et par région. D'un point de vue méthodologique, l'accès direct aux données des différentes exploitations permet d'évaluer l'effet du soutien des prix du marché sur chaque exploitation et de calculer les coefficients de Gini directement à partir de données individuelles. 
Tableau 1.1. Participants au projet relatif à la distribution du soutien et des revenus

\begin{tabular}{|c|c|c|c|}
\hline Pays & Source/contact & $\begin{array}{l}\text { Date de réception } \\
\text { des données }\end{array}$ & Observations $^{1}$ \\
\hline Belgique, Flandre & $\begin{array}{l}\text { Joeri Deuninck, département de } \\
\text { l'Agriculture et de la Pêche }\end{array}$ & $\begin{array}{l}\text { Données reçues en } \\
\text { mars } 2010 \text { et actualisées } \\
\text { en juillet } 2010\end{array}$ & $\begin{array}{l}\text { Données sur la } \\
\text { Flandre }\end{array}$ \\
\hline Canada & $\begin{array}{l}\text { Martin Beaulieu, Statistique Canada } \\
\text { Nathan Niu, Agriculture et Agroalimentaire } \\
\text { Canada }\end{array}$ & $\begin{array}{l}\text { Données reçues en } \\
\text { juin } 2009 \text { et actualisées } \\
\text { en septembre } 2009 \text { et } \\
\text { mai } 2010\end{array}$ & $\begin{array}{l}\text { Données } \\
\text { régionales ; revenu } \\
\text { des ménages } \\
\text { agricoles }\end{array}$ \\
\hline Danemark & $\begin{array}{l}\text { Kim Martin Lind, Institute of Food and } \\
\text { Resource Economics }\end{array}$ & $\begin{array}{l}\text { Données reçues en } \\
\text { octobre } 2010 \text { et } \\
\text { actualisées en novembre } \\
2010\end{array}$ & $\begin{array}{l}\text { Revenu des } \\
\text { ménages agricoles }\end{array}$ \\
\hline Estonie & $\begin{array}{l}\text { Kristel Maidre, ministère de l'Agriculture } \\
\text { Marju Aamisepp, Rural Economy Research } \\
\text { Centre }\end{array}$ & $\begin{array}{l}\text { Données reçues en } \\
\text { novembre } 2009 \text { et } \\
\text { actualisées en février et } \\
\text { juin } 2010\end{array}$ & \\
\hline Finlande & $\begin{array}{l}\text { Olli Voutilainen et Arto Latukka, MTT } \\
\text { Economic Research }\end{array}$ & $\begin{array}{l}\text { Données reçues en } \\
\text { juin } 2010\end{array}$ & $\begin{array}{l}\text { Aucun SPM ; } \\
\text { aucun indice de } \\
\text { Gini }\end{array}$ \\
\hline France & $\begin{array}{l}\text { Frédéric Courleux, Service de la statistique } \\
\text { et de la prospective (SSP), ministère de } \\
\text { l'Agriculture et de la Pêche }\end{array}$ & $\begin{array}{l}\text { Données reçues en } \\
\text { juillet } 2010\end{array}$ & Aucun SPM \\
\hline Allemagne & $\begin{array}{l}\text { Werner Kleinhanss, Heinrich von Thünen- } \\
\text { Institute (vTI) }\end{array}$ & $\begin{array}{l}\text { Données reçues en } \\
\text { mai } 2009 \text { et actualisées } \\
\text { en juillet } 2010\end{array}$ & $\begin{array}{l}\text { Données } \\
\text { régionales }\end{array}$ \\
\hline Irlande & Anne Kinsella et Paul Smyth, Teagasc & $\begin{array}{l}\text { Données reçues en } \\
\text { janvier } 2010 \text { et } \\
\text { actualisées en } \\
\text { octobre } 2010\end{array}$ & $\begin{array}{l}\text { Aucun indice de } \\
\text { Gini }\end{array}$ \\
\hline Italie & Concetta Cardillo, INEA & $\begin{array}{l}\text { Données reçues en } \\
\text { juillet } 2010 \text { et actualisées } \\
\text { en septembre } 2010\end{array}$ & \\
\hline Pays-Bas & $\begin{array}{l}\text { Koen Boone, Agricultural Economics } \\
\text { Research Institute (LEI) }\end{array}$ & $\begin{array}{l}\text { Données reçues en } \\
\text { juillet } 2009 \text { et actualisées } \\
\text { en juin } 2010\end{array}$ & $\begin{array}{l}\text { Revenu des } \\
\text { ménages agricoles }\end{array}$ \\
\hline Espagne & $\begin{array}{l}\text { Secrétariat Général pour les Statistiques } \\
\text { du Ministère de l'Environnement, du Milieu } \\
\text { Rural et Marin }\end{array}$ & $\begin{array}{l}\text { Données reçues en } \\
\text { octobre } 2010\end{array}$ & $\begin{array}{l}\text { Aucun indice de } \\
\text { Gini }\end{array}$ \\
\hline Royaume-Uni & Andrew Woodend, DEFRA & $\begin{array}{l}\text { Données reçues en } \\
\text { février } 2010 \text { et } \\
\text { actualisées en } \\
\text { octobre } 2010\end{array}$ & $\begin{array}{l}\text { Données au niveau } \\
\text { de l'Angleterre ; } \\
\text { données régionales }\end{array}$ \\
\hline États-Unis & $\begin{array}{l}\text { Ron Durst et James MacDonald, Economic } \\
\text { Research Service, USDA }\end{array}$ & $\begin{array}{l}\text { Données reçues en } \\
\text { septembre } 2009 \text { et } \\
\text { actualisées en décembre } \\
2010\end{array}$ & $\begin{array}{l}\text { Données } \\
\text { régionales ; revenu } \\
\text { des ménages } \\
\text { agricoles }\end{array}$ \\
\hline Union européenne & $\begin{array}{l}\text { Bernd Kuepker } \\
\text { DG Agriculture et Développement rural - } \\
\text { Commission européenne }\end{array}$ & $\begin{array}{l}\text { Données reçues en } \\
\text { octobre } 2010\end{array}$ & $\begin{array}{l}\text { Agrégats UE25/27, } \\
\text { UE15, UE10 et } \\
\text { UE2 }\end{array}$ \\
\hline
\end{tabular}

1. Sauf mention contraire, les pays n'ont pas communiqué de données régionales ni d'informations sur le revenu des ménages agricoles.

La section suivante décrit brièvement la méthodologie et les sources de données employées dans cette analyse. Nous présenterons ensuite les principales constatations, avant d'aborder ce que cela implique en termes d'action publique. 


\section{Méthodologie}

Cette étude analyse la distribution du soutien en comparant les différences de soutien moyen observées selon la taille d'exploitation, le type d'exploitations ou la région. Elle indique également la part du soutien total reçue par des catégories données d'exploitations (exploitations laitières ou les $25 \%$ d'exploitations de plus grande taille, par exemple).

Pour mettre en lumière l'effet redistributif du soutien, la distribution de celui-ci est comparée à celles de la production agricole brute et des revenus (variables définies dans l'encadré 2.1 et le paragraphe qui le précède). En outre, les différences de niveau/taux du soutien moyen octroyé à des groupes spécifiques d'exploitations sont comparées aux différences dans le niveau de revenu. Comme l'explique l'encadré 2.2, la relation entre soutien et revenu est purement statique et comptable. Cela ne signifie pas pour autant qu'en termes économiques, toutes les formes de soutien débouchent sur une augmentation du revenu net des agriculteurs. La plupart ont une incidence sur l'utilisation d'intrants ou sur les prix, de sorte qu'une partie du soutien est captée par les fournisseurs d'intrants et la filière alimentaire en aval. Lorsqu'il est associé à la production effective, le soutien aux agriculteurs est absorbé, pour une part importante, dans le « coût des ressources », créant des distorsions dans l'allocation des ressources. De plus, la structure des filières alimentaires peut conduire à une transmission imparfaite des prix, qui fait qu'une partie du soutien bénéficie à la filière en aval. En conséquence, l'efficacité de transfert du revenu de la plupart des types de soutien est inférieure à 1 (OCDE, 2003). Par ailleurs, cette analyse statique ne tient pas compte des ajustements auxquels procèderaient les marchés et les ménages agricoles si le soutien était supprimé. Nous ne pouvons pas déduire de la relation entre soutien et revenu qu'en cas de suppression du premier, le deuxième diminuerait d'un montant équivalent.

Pour la présente analyse, un certain nombre de variables et d'indicateurs ont été calculés à partir de données recueillies à l'échelle des exploitations. Dans la mesure du possible, les définitions des types et groupes d'exploitations ont été harmonisées entre les pays, de même que les définitions des variables (revenu agricole, par exemple) et des indicateurs (tels que les indices de Gini) utilisés dans l'analyse. Ces efforts permettent d'améliorer la comparabilité entre pays mais pas d'atteindre une cohérence parfaite car on continue à recourir à certaines définitions nationales.

\section{Définition de la population et des agrégats}

Le réseau a permis de renforcer la cohérence des définitions entre les pays. Un problème majeur se pose lorsque l'on compare les statistiques des exploitations de l'Union européenne avec celles d'Amérique du Nord ou d'Asie, car l'Union européenne définit les exploitations de manière restrictive, de sorte qu'un grand nombre des plus petites d'entre elles sont exclues de l'analyse, tandis que d'autres pays adoptent des limites très basses de taille d'exploitation, ce qui a pour effet d'inclure les activités agricoles à temps partiel ou de loisir. La définition retenue pour qualifier une «exploitation» influant fortement sur la distribution, il était essentiel de réduire les différences pour pouvoir établir des comparaisons. Il a donc été décidé d'exclure les plus petites fermes à condition que la population totale d'exploitations de l'échantillon représente $90 \%$ des ventes totales de produits agricoles. À l'opposé, aucune limite maximale n'a été imposée, et les exploitations constituées en société ont été incluses (excepté pour certaines variables telles que le revenu non agricole). 
Les exploitations de la population sont réparties en quartiles définis en fonction de la production agricole brute. On dispose ainsi d'informations sur la part du soutien reçue par les $25 \%$ d'exploitations les plus grandes, par exemple.

S'agissant des types d'exploitations, il a été décidé de conserver les définitions nationales et d'effectuer les regroupements suivants :

- Grandes cultures (de plein champ)

- Pépinières/cultures en serre

- Cultures fruitières et maraîchères

- Élevage laitier

- Élevage bovin (à l'herbe et en parc d'engraissement)

- Élevage porcin

- Élevage avicole (volailles/œufs)

- Élevage ovin/caprin, et

- $\quad$ Autres

De nombreuses exploitations ont une production diversifiée. Les exploitations sont généralement classées dans la catégorie d'activité dont le produit représente la plus grande part du chiffre d'affaire (Canada et États-Unis) ou de la marge brute (marge brute standard dans le RICA de l'Union européenne). Dans certains pays membres de l'Union européenne, une partie non négligeable des exploitations est classée dans la catégorie "autres" qui comprend généralement des exploitations mixtes sans spécialisation nette.

Quant aux régions, les participants ont décidé du niveau régional approprié pour leur pays, mais il leur a été suggéré d'utiliser la définition des régions rurales, intermédiaires et urbaines ${ }^{5}$ établie par l'OCDE.

\section{Définition des variables et des indicateurs}

De manière générale, les variables et les indicateurs ont été calculés à l'aide de données individuelles. Les participants ont fourni une série de tableaux standard (annexe 1) contenant les variables relatives aux comptes des exploitations qui sont énumérées et définies dans l'encadré 2.2. Il a été décidé en particulier :

- de présenter deux indicateurs du revenu agricole (recettes brutes moins charges variables et fixes), avant et après amortissements ;

- d'inclure le revenu non agricole lorsque celui-ci était disponible ;

- d'utiliser les estimations nationales de l'amortissement ;

- d'exclure les taxes sur la valeur ajoutée, le cas échéant ;

- d'inclure le soutien des prix du marché (SPM), en utilisant les taux fournis par l'OCDE pour une liste de produits de base et en formulant les hypothèses appropriées pour les produits pour lesquels le soutien des prix du marché n'était pas calculé ;

- de distinguer, d'une part, les paiements tels que ceux effectués au titre du second pilier de la Politique agricole commune dans l'Union européenne, et,

5. Pour plus d'informations sur cette typologie, voir le rapport OCDE (2009a), qui s'intéresse à la part de l'agriculture dans l'économie des régions rurales. 
d'autre part, les paiements octroyés au titre des programmes de produits ou du premier pilier ;

- de présenter les résultats dans la monnaie nationale, le cas échéant ; et

- d'inclure des informations sur les actifs agricoles.

\section{Encadré 2.1. Liste et définition des variables des tableaux standard}

Nombre d'exploitations couvertes (représentant $90 \%$ des ventes totales de produits agricoles)

Nombre d'unités de travail (équivalent temps plein)

Nombre d'hectares de superficie agricole utilisée (SAU)

La production agricole brute $(\mathrm{PAB}$ ) inclut les recettes (chiffre d'affaire) tirées des ventes de produits et de services agricoles, tels que le travail à façon ou la mise en location de terres, de quotas, de bâtiments ou de machines, entre autres. Dans le Réseau d'information comptable agricole (RICA) de l'Union européenne, la production agricole brute comprend les ventes et l'autoconsommation de produits (végétaux et animaux) et d'animaux, la variation des stocks de produits (végétaux et animaux), la variation de la valeur d'inventaire des animaux (moins les achats d'animaux) et les produits divers non exceptionnels.

Le soutien total (souvent désigné par le terme "soutien » dans le texte) inclut l'ensemble des paiements et le soutien des prix du marché.

La ligne tous paiements comprend les transferts budgétaires aux agriculteurs résultant de la politique agricole et des paiements des systèmes d'assurance, des programmes de conservation des terres aux États-Unis et des premier et second piliers de la Politique agricole commune de l'Union européenne. On distingue trois catégories : les paiements au titre du premier pilier, y compris les paiements directs au revenu; les paiements au titre du second pilier, ce qui comprend les paiements reçus dans le cadre de la politique de développement rural de la Politique agricole commune ; et les autres paiements, provenant principalement de dépenses nationales.

Le soutien des prix du marché (SPM) comprend les transferts des consommateurs et contribuables aux producteurs agricoles découlant des mesures qui créent un écart entre le prix du marché intérieur et le prix à la frontière d'un produit agricole donné, écart mesuré au départ de l'exploitation. Le SPM est calculé dans la base de données des estimations du soutien aux producteurs (ESP) pour les principaux produits de base ; gérée par I'OCDE, cette base couvre en principe au moins $70 \%$ de la valeur totale de la production agricole. Pour chaque produit, le SPM est égal à la différence, mesurée au départ de l'exploitation, entre le prix intérieur à la production $(\mathrm{PP})$ et un prix à la frontière (PF), multipliée par la quantité produite (QP).

$$
S P M_{i}=\left(P P_{i}-P F_{i}\right){ }^{*} Q P_{i} \text {. pour le produit } \mathrm{i}
$$

Le SPM par produit peut être exprimé comme une part de la valeur de la production de ce produit (VP).

Ratio SPM pour le produit $\mathrm{i}=\mathrm{SPM}_{\mathrm{i}} / \mathrm{VP}_{\mathrm{i}}$

Le SPM est calculé au niveau de l'exploitation en appliquant le ratio SPM de chaque produit aux recettes correspondantes générées dans l'exploitation. Pour les recettes relatives aux autres produits, on utilise le ratio SPM moyen de l'ensemble des produits de la base ESP. Le SPM par exploitation est la somme des SPM des différents produits de cette exploitation, calculés comme indiqué ci-dessus.

Les recettes brutes comprennent la production agricole brute et l'ensemble des paiements.

Les dépenses d'exploitation s'entendent hors dotations aux amortissements. Elles incluent l'ensemble des dépenses relatives aux cultures, à l'élevage et aux machines ainsi que les dépenses courantes, telles que les charges d'intérêts nettes, les salaires, les locations, les primes d'assurance, le téléphone, l'électricité, le carburant, les travaux à façon et la location de machines, les impôts fonciers nets, les réparations de bâtiments et de clôtures, les frais de transport (notamment par camion), les frais de vente, les redevances payées aux offices de commercialisation, les frais de comptabilité, etc.

Le revenu net d'exploitation est la différence entre les recettes brutes et les dépenses d'exploitation, et correspond également au revenu agricole avant amortissements.

Les amortissements comprennent l'estimation nationale de l'amortissement économique ou la déduction fiscale pour amortissement, c'est-à-dire le montant déduit du revenu imposable pour tenir compte du coût annuel de l'amortissement à un taux spécifique au matériel amorti.

Le revenu agricole correspond au revenu net d'exploitation diminué des dotations aux amortissements. 
Le revenu non agricole comprend le revenu tiré des activités exercées hors de l'exploitation par les exploitants et les autres membres des ménages. En sont exclus les revenus non agricoles des ménages dirigeant une exploitation constituée en société.

Le revenu des ménages agricoles comprend les revenus agricole et non agricole.

Les relations entre les composantes du revenu apparaissent également dans le diagramme ci-après.

\section{Composantes du revenu des ménages agricoles}
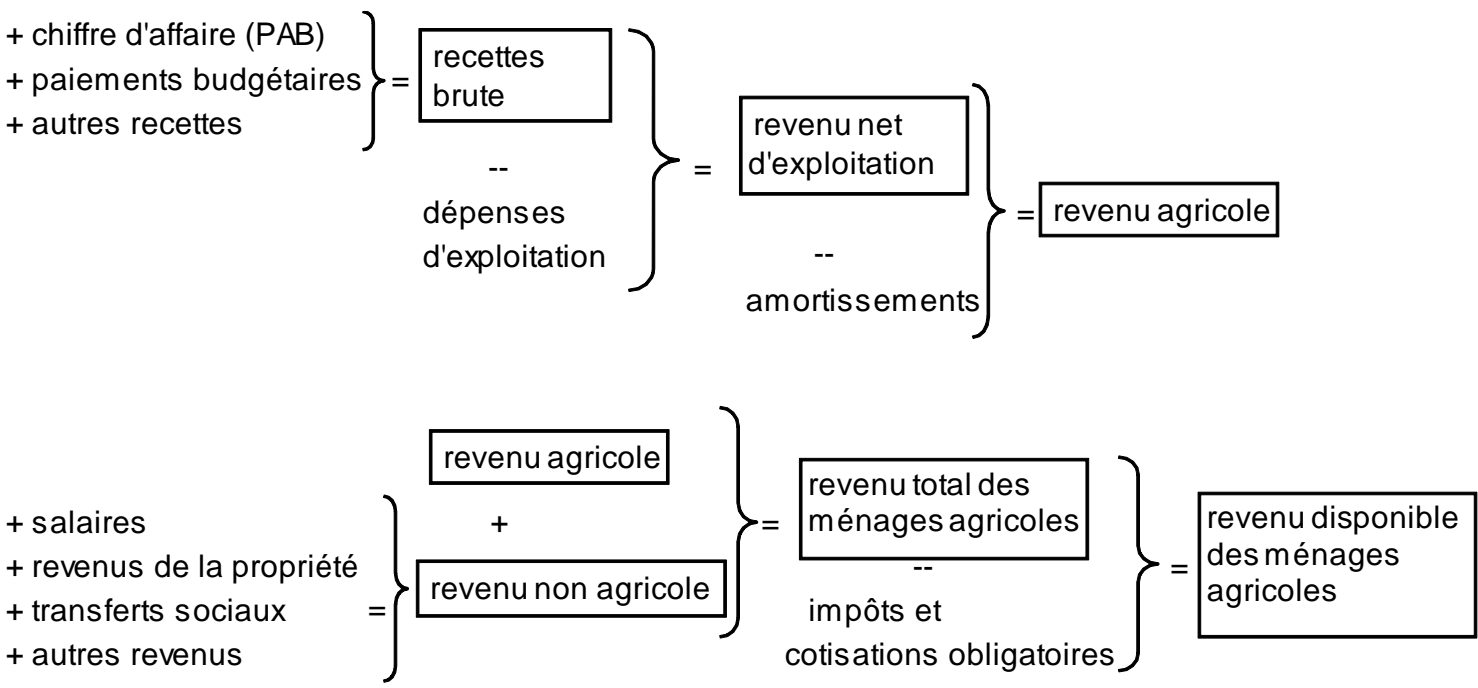

Suite à la quatrième réunion du réseau, les tableaux standard ont été revus afin d'y inclure les variables supplémentaires ci-après.

Le total des actifs agricoles comprend la valeur marchande à la fin de l'année civile des animaux pour la boucherie et l'engraissement et des animaux de reproduction et de remplacement, les comptes clients, les stocks d'intrants, les dépenses d'intrants payées d'avance, les cultures destinées à la vente, les investissements agricoles, les terres et les bâtiments agricoles détenus en pleine propriété (y compris les locaux d'habitation), les machines et le matériel détenus en pleine propriété, le quota de production.

Le total des dettes agricoles comprend l'endettement à court et à long terme envers les banques, les sociétés de gestion de portefeuilles, les coopératives de crédit et les organismes publics; les sommes perçues à titre d'acomptes ou d'avances ; les sommes dues aux fournisseurs de matériel et de consommables, à des particuliers et aux actionnaires ; et les comptes créditeurs à la fin de l'année civile.

Les capitaux propres agricoles correspondent à la différence entre le total des actifs agricoles et le total des dettes agricoles.

Le taux de soutien est le ratio du soutien total sur la production agricole brute.

Les données fournies par les participants au réseau peuvent être utilisées pour comparer le niveau moyen de soutien ou de revenu d'un groupe d'exploitations avec la moyenne pour l'ensemble des exploitations. Les indices de Gini, qui mesurent l'égalité de la distribution d'une variable, ont été calculés directement par les participants à l'aide d'informations recueillies au niveau des exploitations. Des courbes de concentration peuvent être créées à partir des quartiles. L'encadré 2.2 fournit la définition et la formule de ces deux mesures. 
Une courbe de concentration représente la proportion cumulée d'une variable en fonction de la part de la population contribuant à (prise en compte dans) cette variable. Les axes varient entre 0 et 1 et la première diagonale est la ligne d'égalité. La distance entre la courbe de la variable et la ligne d'égalité indique le degré d'inégalité de la distribution de la variable. Plus la courbe de la variable s'éloigne de la ligne d'égalité, plus la concentration de la variable est forte et plus sa distribution est inégale.

La distance peut être mesurée par un indice de Gini. Ce coefficient correspond au ratio de l'aire comprise entre la ligne d'égalité (première diagonale) et la courbe de concentration sur l'aire totale sous la diagonale. S'il est calculé à l'aide de données individuelles, il varie entre 0 et 1 . Un faible indice de Gini indique une distribution plus égalitaire, 0 correspondant à l'égalité parfaite, tandis qu'un indice élevé traduit une distribution plus inégalitaire, 1 correspondant à l'inégalité absolue. Les progiciels statistiques incluent souvent une fonction permettant de calculer des indices de Gini.

\section{Années}

Les participants ont fourni les informations relatives à l'année 2004, ce qui dans l'Union européenne correspond à la situation d'avant la mise en œuvre de la réforme de la Politique agricole commune de 2003, et aux années 2006 et 2007. Malgré les variations de revenus d'une année sur l'autre, nous n'avons pas jugé approprié de faire porter l'analyse sur une période de trois ans, car cette période aurait inclus, pour l'Union européenne, la mise en œuvre d'une réforme importante.

\section{Pays}

Les participants de treize pays et régions (Allemagne, Angleterre, Canada, Danemark, Espagne, Estonie, Finlande, Flandres belges, France, Irlande, Italie, Pays-Bas et États-Unis) et la Commission européenne ont fourni des données (tableau 1.1). Les données de la Commission européenne portent sur quatre agrégats de l'Union européenne : UE27 correspond à l'ensemble des États membres de l'Union européenne, c'est-à-dire UE25 pour 2004 et 2006 et UE27 pour 2007; UE15 regroupe les États membres en 2003 ; UE10, les nouveaux États membres ayant rejoint l'Union en 2004 ; et UE2, la Bulgarie et la Roumanie, devenues membres en 2007.

\section{Sources de données}

Pour chaque pays, deux types de sources de données sont nécessaires pour calculer la distribution du soutien et la comparer à celle d'autres variables relatives aux comptes des exploitations, telles que la production ou les revenus :

- Données au niveau des exploitations : ces données ont été recueillies lors d'enquêtes menées sur les comptes des exploitations et comprenant toutes les variables nécessaires pour calculer le revenu agricole (les recettes de l'exploitation, y compris le chiffre d'affaires et les subventions, les dépenses d'exploitation, les dotations aux amortissements, etc.) et d'autres informations structurelles. Pour certains pays, des informations sur le revenu non agricole ont également été fournies. Ces données proviennent du Réseau d'information comptable agricole (RICA) ${ }^{6}$ pour les États membres de l'Union européenne, de l'enquête ARMS (Agricultural Resource Management

6. Voir la documentation de l'Union européenne relative au réseau RICA à l'adresse ec.europa.eu/agriculture/rica/methodology2_fr.cfm. 
Survey, enquête de gestion des ressources agricoles) aux États-Unis ${ }^{7}$, et de fichiers de données fiscales et de données issues de l'Enquête financière sur les fermes au Canada. Consultez l'annexe B pour obtenir des informations détaillées par pays.

- Base de données des ESP : les informations sur le soutien des prix du marché en pourcentage de la valeur de la production proviennent de la base de données des estimations du soutien aux producteurs $(\mathrm{ESP})^{8}$ gérée par l'OCDE. Le SPM calculé pour un certain nombre de produits de base correspond à l'écart entre le prix du marché intérieur et le prix à la frontière, multiplié par le niveau de production intérieure. Le SPM d'un produit donné est exprimé sous la forme d'un pourcentage de la valeur de la production de ce produit. Ce pourcentage est ensuite appliqué au chiffre d'affaires réalisé dans chaque exploitation de l'enquête pour le produit considéré (encadré 2.1).

\section{Principaux résultats}

L'analyse vise à éclairer la façon dont le soutien se répartit entre les exploitations agricoles et dont cette distribution se compare à celle des composantes du revenu. $\mathrm{Ce}$ rapport fournit des informations sur la concentration de ce soutien et décrit les inégalités observées dans sa distribution et les compare à celle de la production agricole brute, des recettes brutes, des revenus et des actifs. Il expose également les différences entre le niveau moyen de soutien et de revenu de divers sous-ensembles d'exploitations agricoles (regroupées par taille d'exploitation, type d'exploitations et type de régions) et la moyenne de l'ensemble des exploitations, et examine les variations de la part du soutien dans les recettes agricoles selon le type d'exploitations. Enfin, il étudie les inégalités générales de distribution du soutien et d'autres composantes du revenu au moyen d'indices de Gini. La définition des variables employées dans ce rapport est fournie à l'encadré 2.1. Utilisés seuls, le terme «soutien» désigne le soutien total et le terme «paiements », le total des paiements.

\section{Distribution du soutien, des revenus et des actifs par taille d'exploitation}

Dans la plupart des pays considérés, les $25 \%$ d'exploitations les plus grandes représentent entre $60 \%$ et $80 \%$ de la production agricole brute (cette proportion est plus forte en Estonie et moindre au Danemark et en Italie) et entre $50 \%$ et $75 \%$ du soutien total de l'ensemble des exploitations, y compris le soutien des prix du marché (graphique 3.1, tableau $\mathrm{C} 1$ en annexe) ${ }^{9}$. À l'inverse, les $25 \%$ d'exploitations les plus petites ne représentent que $2 \%$ de la production agricole brute en Estonie, en Irlande et dans la zone UE27; leur part peut monter jusqu'à $13 \%$ au Danemark et en Italie. Elles

\footnotetext{
7. Voir la documentation relative à l'ARMS à l'adresse www.ers.usda.gov/Data/ARMS/GlobalDocumentation.htm.
}

8. Les données des ESP sont librement accessibles sur le site Web de l'OCDE à l'adresse www.oecd.org/agriculture/pse. Ce site fournit également des informations sur la méthodologie utilisée et l'interprétation des indicateurs de soutien de l'OCDE.

9. Les $25 \%$ d'exploitations les plus grandes sont celles figurant dans le quartile supérieur, établi à partir de la production agricole brute. 
reçoivent entre $4 \%$ et $13 \%$ environ du soutien total dans la plupart des pays (graphique 3.2, tableau $\mathrm{C} 2$ en annexe). En raison de 1'homogénéité de ses structures agricoles et des caractéristiques de la spécialisation de sa production, l'Italie fait figure d'exception, car les $25 \%$ d'exploitations les plus petites y reçoivent $22.5 \%$ du soutien total et $25 \%$ du total des paiements, tandis que les $25 \%$ d'exploitations les plus grandes y bénéficient de $35 \%$ du soutien total et de $33 \%$ du total des paiements. Ces chiffres indiquent que le soutien est moins concentré que la production agricole brute dans la plupart des pays. Les graphiques 3.1 et 3.2 font également apparaître que la concentration de la production agricole brute varie considérablement selon les pays, les niveaux de concentration les plus élevés se trouvant en Estonie et dans la zone UE2 $7^{10}$.

Le soutien des prix du marché (SPM) profite généralement davantage aux grandes exploitations que les paiements car il est directement lié à la production (graphique 3.1). Cette tendance peut également être représentée de manière graphique en utilisant des courbes de concentration réalisées sur la base des quartiles établis à partir de la production agricole brute (encadré 2.2). Le graphique 3.3 montre que le total des paiements est plus proche que le SPM de la première diagonale (qui figure l'égalité parfaite).

On constate des différences importantes entre la distribution des paiements et celle du SPM parmi les États membres de l'Union européenne, différences qui correspondent à celles observées dans la structure de la production et du soutien. En Allemagne, l'écart est faible car les paiements du premier pilier ${ }^{11}$, qui représentent plus des trois quarts des paiements, suivent de près la distribution du SPM. La mise en œuvre du régime de paiement unique (RPU) en 2005 a profondément transformé la concentration des paiements effectués dans le cadre du premier pilier. Bien que la plupart des paiements ne soient plus corrélés à la superficie réelle exploitée ni à la taille effective du cheptel, ils restent liés aux aides accordées par le passé (mise en œuvre historique) ou à la surface gérée par l'exploitant (mise en œuvre régionale). En Italie, la distribution des paiements est également très similaire à celle du SPM. En Estonie, les paiements du premier pilier, versés selon un régime de paiement unique à la surface (RPUS) avec le même taux par hectare pour une même année, sont distribués de manière plus inégale que le SPM. Étant donné que le taux des paiements augmente chaque année pendant la période de transition de dix ans suivant l'adhésion des pays qui ont rejoint l'Union européenne en 2004, il faut s'attendre à ce que les écarts dans la distribution du soutien total se creusent. Le constat est le même pour la zone UE2, qui regroupe la Bulgarie et la Roumanie.

Dans les agrégats de l'Union européenne et dans les États membres de l'Union inclus dans l'analyse, les paiements du second pilier sont distribués de manière plus égale entre les exploitations agricoles de taille différente, en particulier en Allemagne où les $25 \%$ d'exploitations les plus grandes reçoivent $34 \%$ de ces paiements (contre près de $60 \%$ des paiements du premier pilier). En 2007, les paiements du second pilier ont représenté $40 \%$ des paiements en Estonie, $20 \%$ en Angleterre, et environ $15 \%$ en Allemagne et $10 \%$ aux

10. En fait, la diversité au sein de l'Union européenne s'est accentuée avec l'élargissement, si bien que la concentration de la production agricole brute est supérieure dans la zone UE27 que dans les sous-groupes de l'Union européenne (UE15, UE10 et UE2).

11. Pour obtenir un aperçu général de la Politique agricole commune, voir OCDE (2011). 
Pays-Bas. La majorité des paiements du second pilier sont ciblés sur des résultats environnementaux précis, des régions présentant certains handicaps ou des projets de développement rural particuliers (OCDE, 2009b). Ces paiements ont donc moins de chances d'être proportionnels à la taille des exploitations. Dans la plupart des États membres de l'Union européenne, les paiements du second pilier sont moins concentrés que ceux du premier, et les uns comme les autres sont moins concentrés que le SPM, sauf en Flandre belge et en Italie (graphique 3.4). En France, les paiements du second pilier concernent principalement les petites exploitations agricoles : les $25 \%$ d'exploitations les plus petites reçoivent environ $35 \%$ des paiements octroyés au titre du second pilier.

De façon générale, le soutien se répartit inégalement entre les exploitations de différentes tailles, mais dans la plupart des cas, cette inégalité est moindre que dans le cas de la production agricole brute ou des recettes agricoles. Ceci indique que, si l'on considère la taille des exploitations, le soutien a un effet redistributif. En outre, celui-ci est supérieur sous forme de paiements que sous forme de SPM. Comme on peut le voir sur le graphique 3.3, le revenu agricole est généralement distribué de manière plus égale que la production agricole brute, mais cette différence n'est pas uniquement liée au soutien.

La plupart des pays ne communiquent pas d'informations sur le revenu non agricole des ménages agricoles, soit parce qu'ils ne collectent pas ces données, soit parce que les résultats ne sont pas représentatifs faute de réponses. Dans les quatre pays qui font état du revenu total des ménages agricoles, il est clair que la rémunération des activités exercées hors de l'exploitation réduit les inégalités de revenu par taille d'exploitation, car les plus petites fermes tendent à se reposer davantage sur ce type de rentrées que les plus grandes (graphique 3.3).

Dans les différents pays de l'Union européenne qui ont fourni ces renseignements et au Canada, la distribution des dettes agricoles est assez similaire à celle du revenu agricole, tandis que dans les agrégats de l'Union européenne, elle est plus inégale. Par ailleurs, la distribution des capitaux propres est plus égale que celle des dettes dans tous les pays (graphique 3.5). Cela tendrait à montrer que les exploitations plus petites sont proportionnellement moins endettées que les grandes. 


\section{Graphique 3.1. Part des $25 \%$ d'exploitations les plus grandes dans certains pays de l'OCDE,} par composante du revenu, 2007
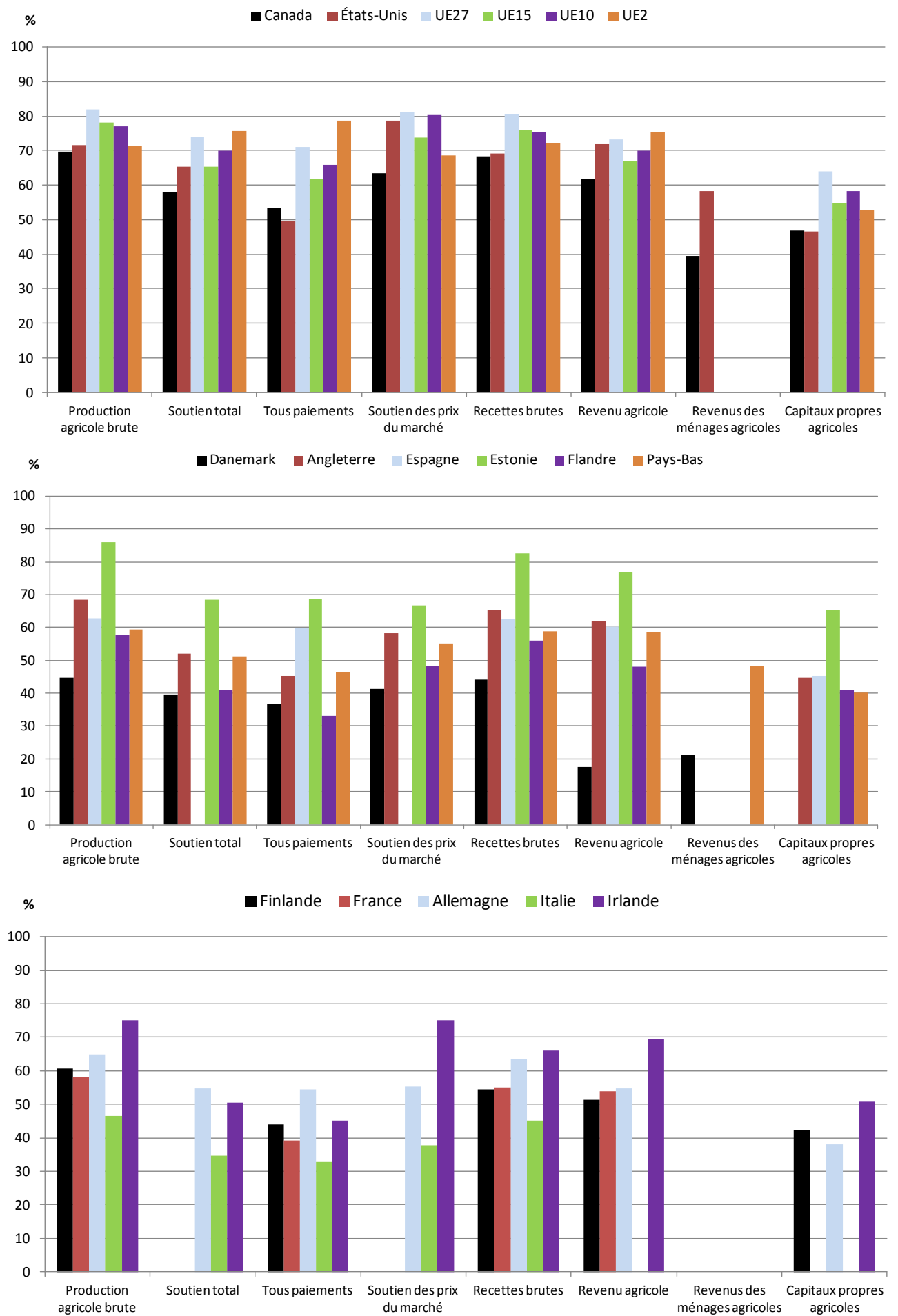

Les limites des quartiles sont établies à partir de la production agricole brute. En Italie, le revenu agricole n'est pas inclus en raison de la présence de nombres négatifs. La distribution du SPM n'est pas disponible pour la Finlande et la France.

Source et notes : tableau $\mathrm{C} 1$ en annexe. 
Graphique 3.2. Part des $25 \%$ d'exploitations les plus petites dans certains pays de l'OCDE, par composante du revenu, 2007

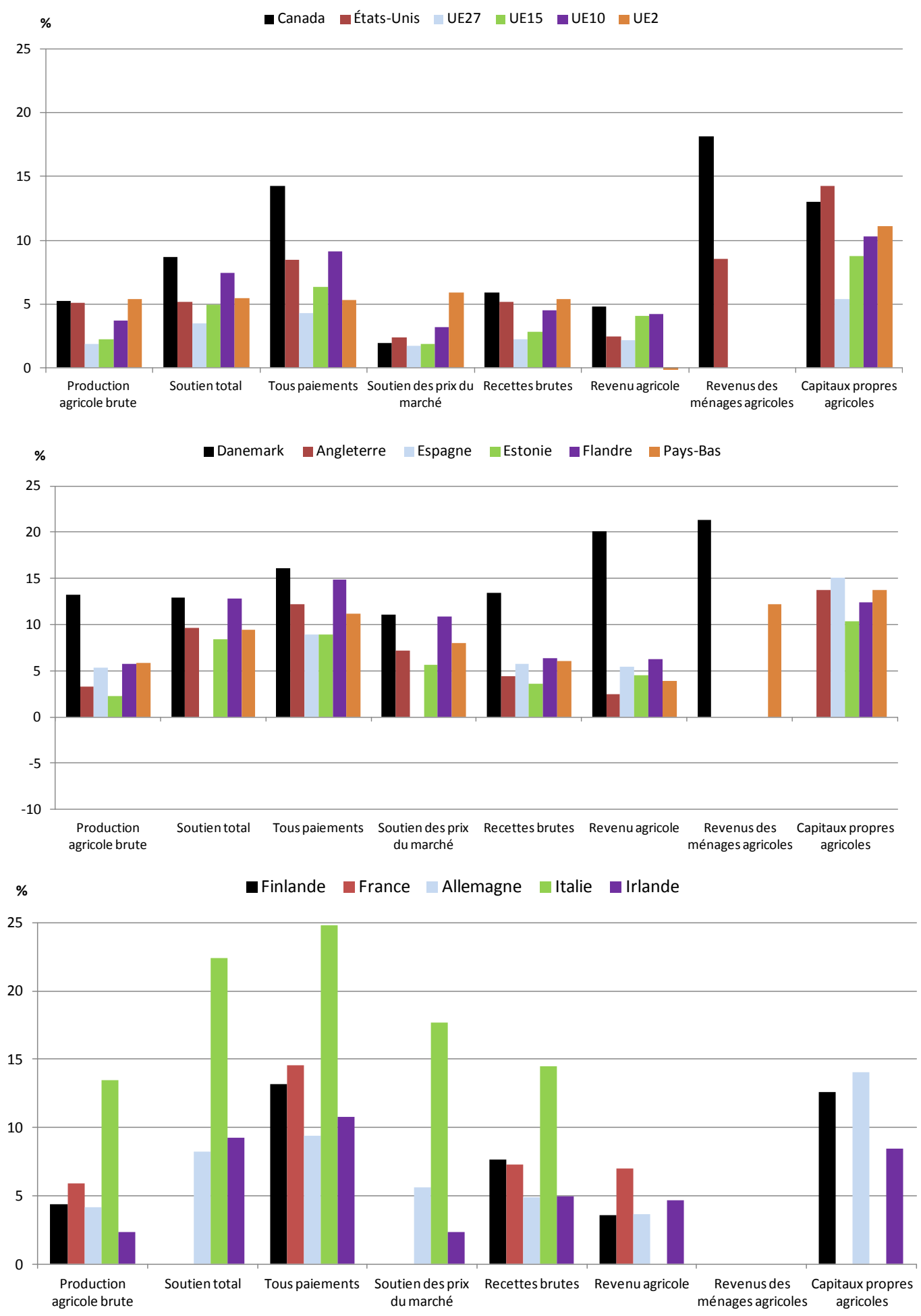

Les limites des quartiles sont établies à partir de la production agricole brute. En Italie, le revenu agricole n'est pas inclus en raison de la présence de nombres négatifs. La distribution du SPM n'est pas disponible pour l'Espagne, la Finlande et la France.

Source et notes : tableau $\mathrm{C} 2$ en annexe. 
Graphique 3.3. Courbes comparant la distribution des composantes du soutien et du revenu, 2007
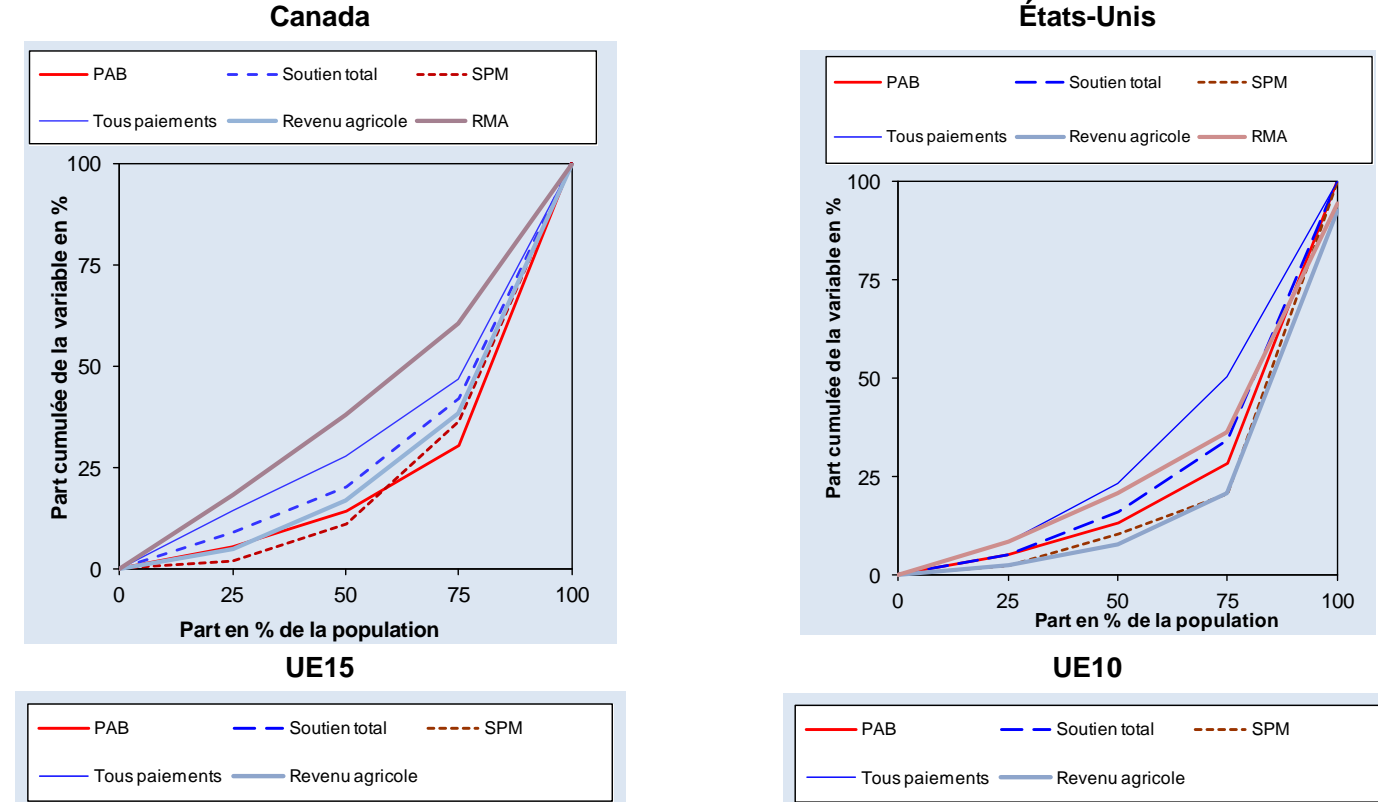

UE10
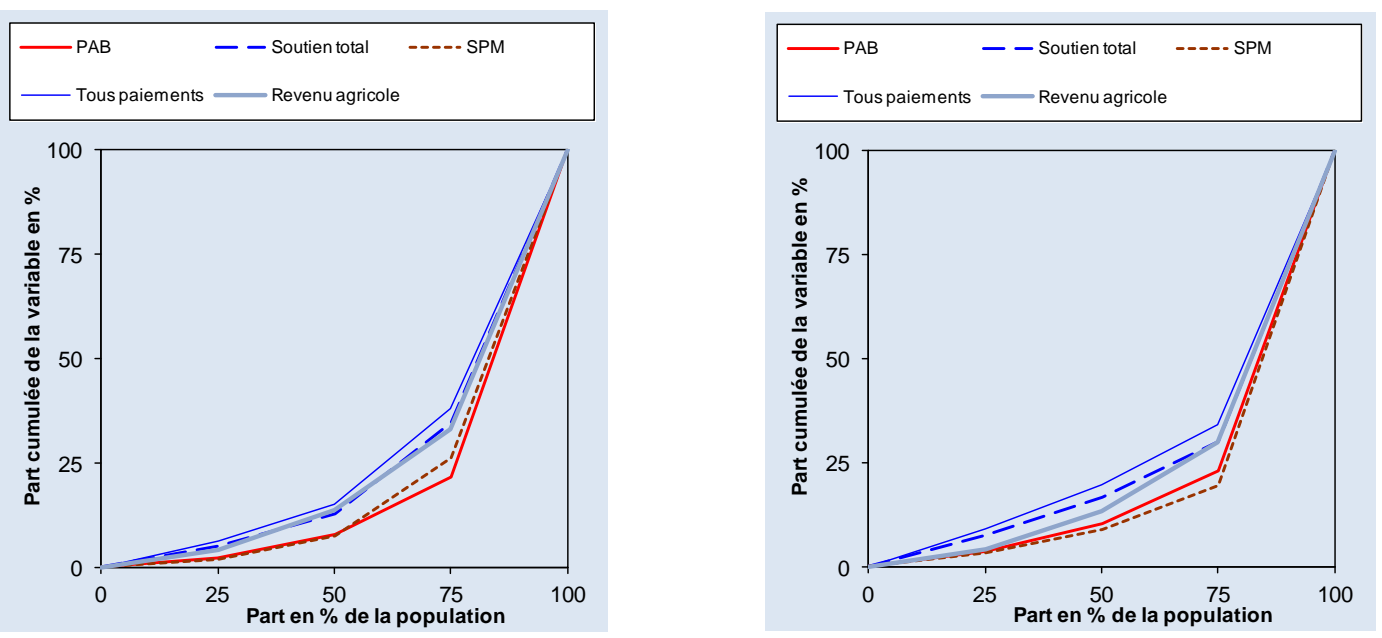

UE27

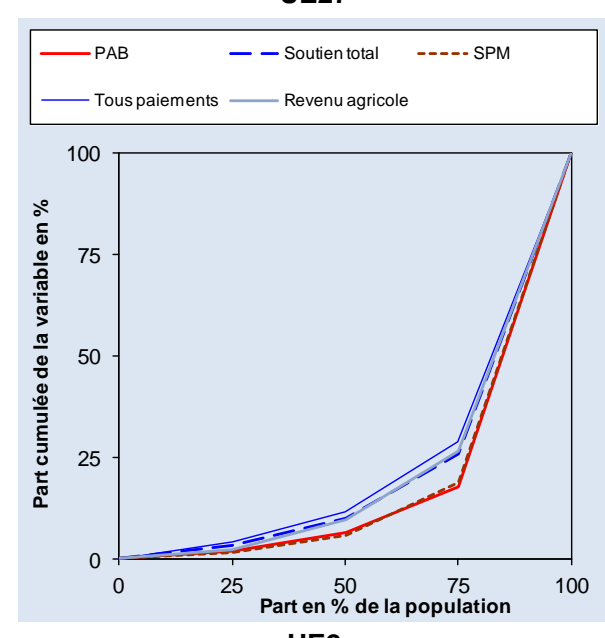

UE2
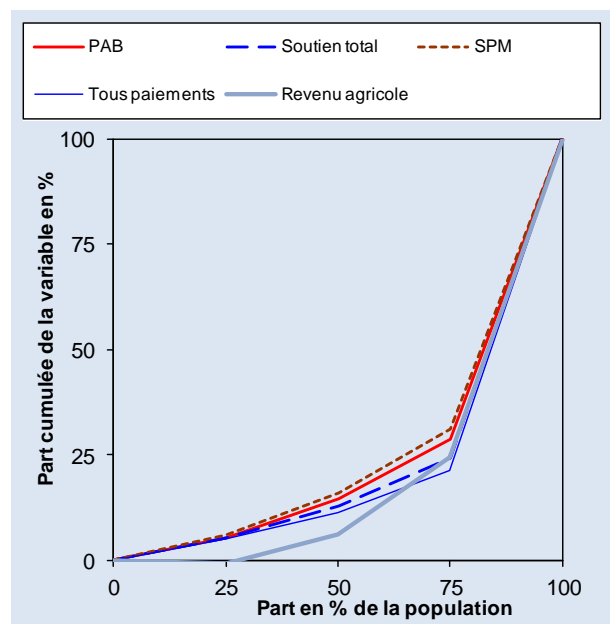
Graphique 3.3. Courbes comparant la distribution des composantes du soutien et du revenu, 2007 (suite)

Danemark
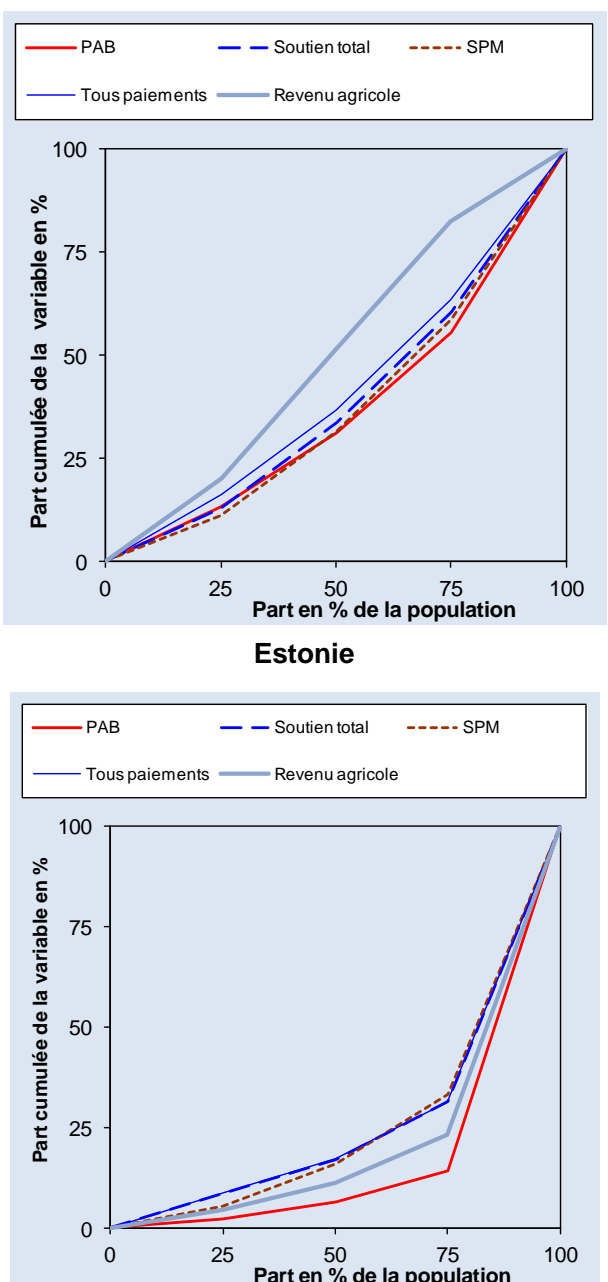

Angleterre
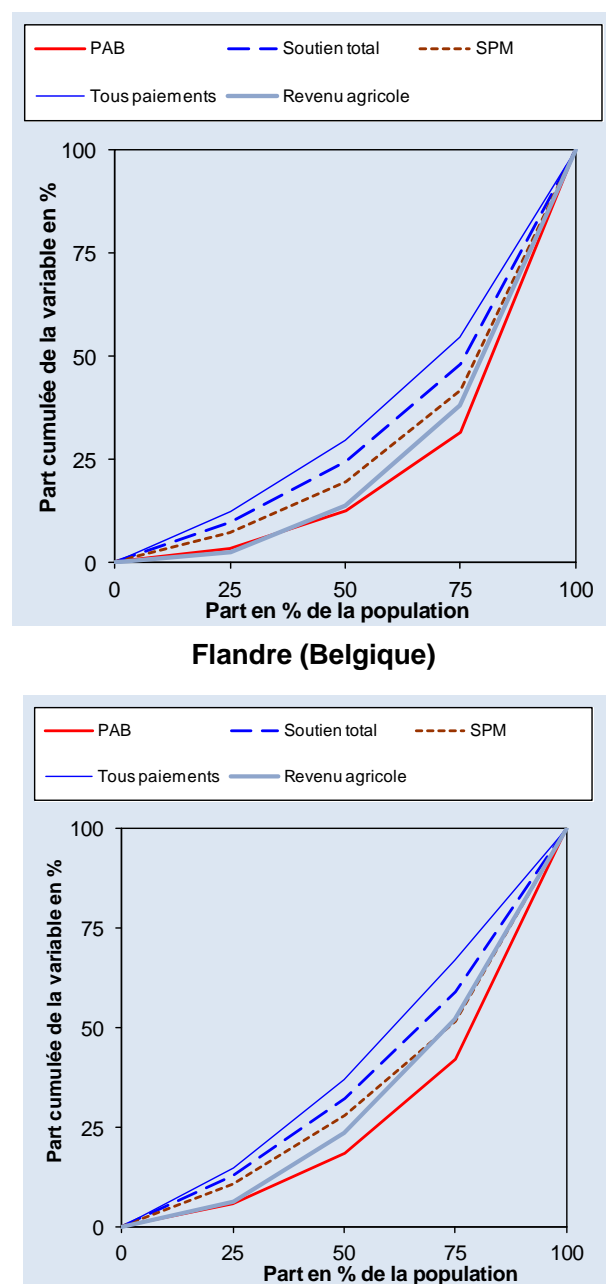

Espagne

— $\mathrm{PAB}$ - Tous paiements - Revenu agricole
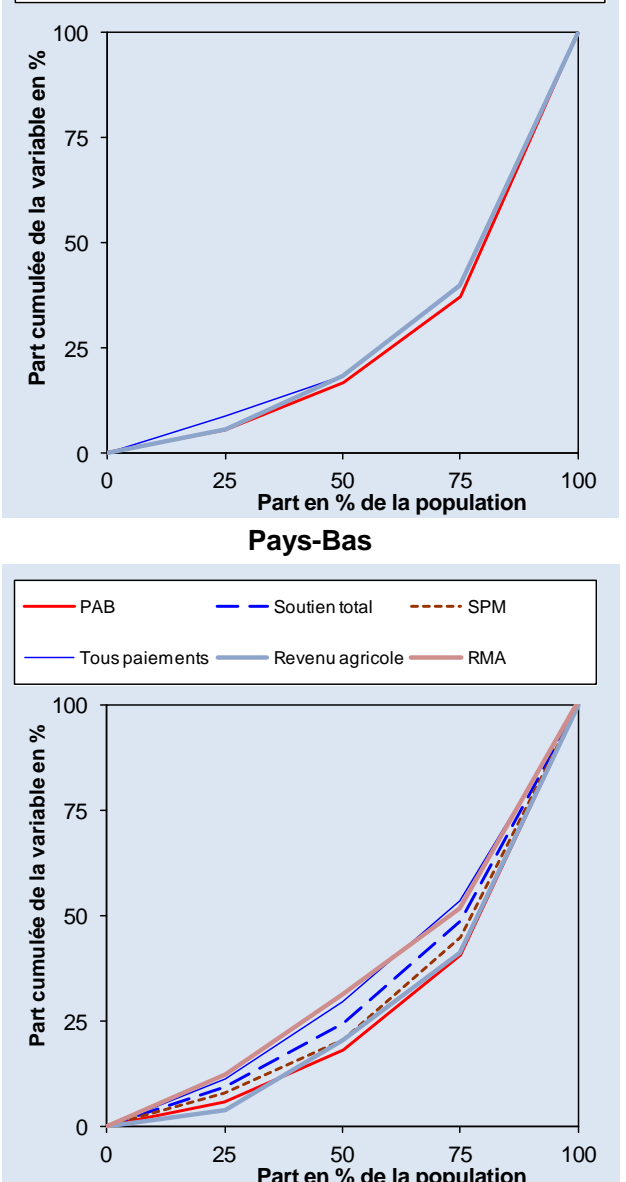
Graphique 3.3. Courbes comparant la distribution des composantes du soutien et du revenu, 2007 (suite)

\section{Finlande}

— PAB — Touspaiements - Revenuagricole

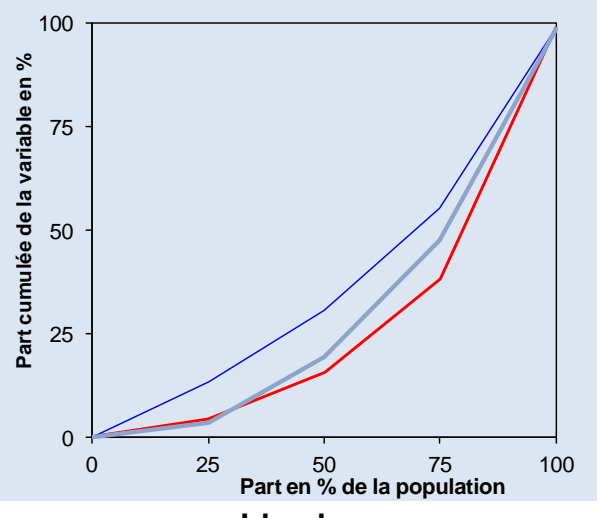

Irlande
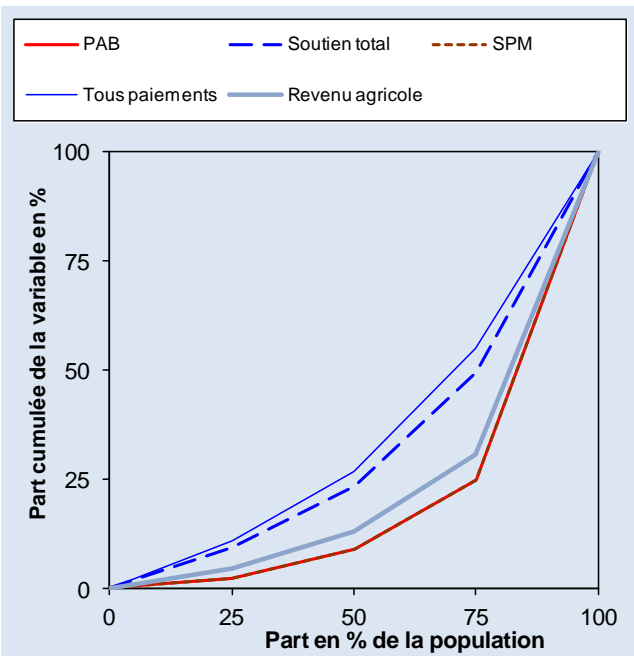

France

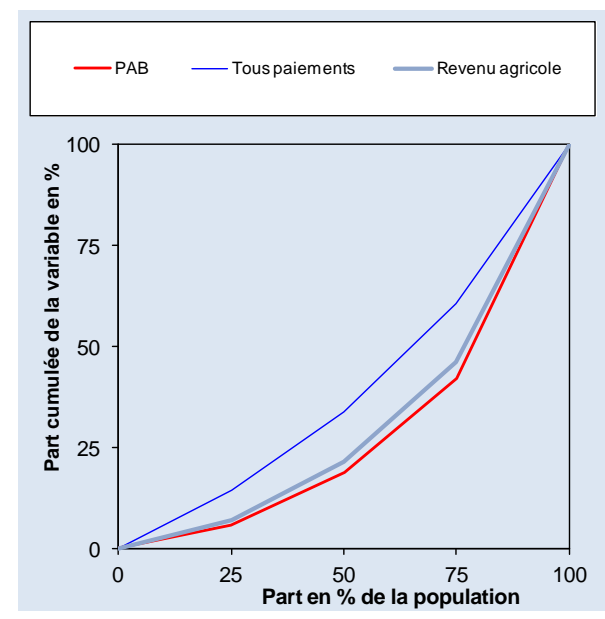

Italie
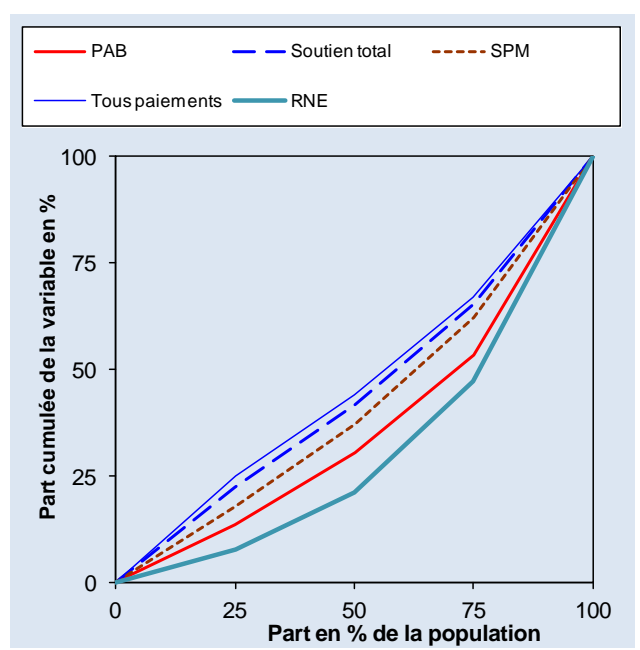

Allemagne
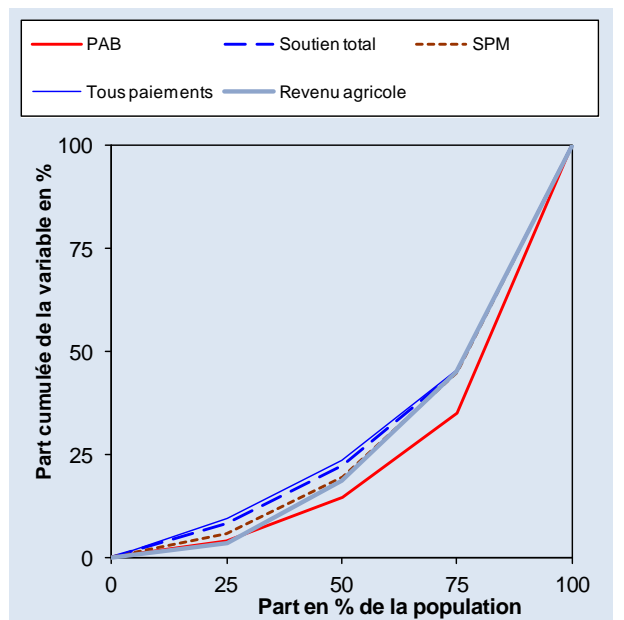

Les courbes sont réalisées sur la base des quartiles établis à partir de la production agricole brute (PAB); SPM : soutien des prix du marché; population: exploitations agricoles représentées par l'échantillon. En Italie, le revenu agricole n'est pas inclus en raison de la présence de nombres négatifs. Pour l'Espagne, la Finlande et la France, le SPM n'est pas disponible et le soutien total n'est pas indiqué. Les variables sont définies dans les notes du tableau $\mathrm{C} 1$ en annexe.

Source : tableaux transmis par les participants. 
$\mathbf{2 4}$ - DisTRIBUTION DU SOUTIEN ET DES REVENUS DANS L'AGRICULTURE

Graphique 3.4. Courbes comparant la distribution des composantes du soutien, 2007
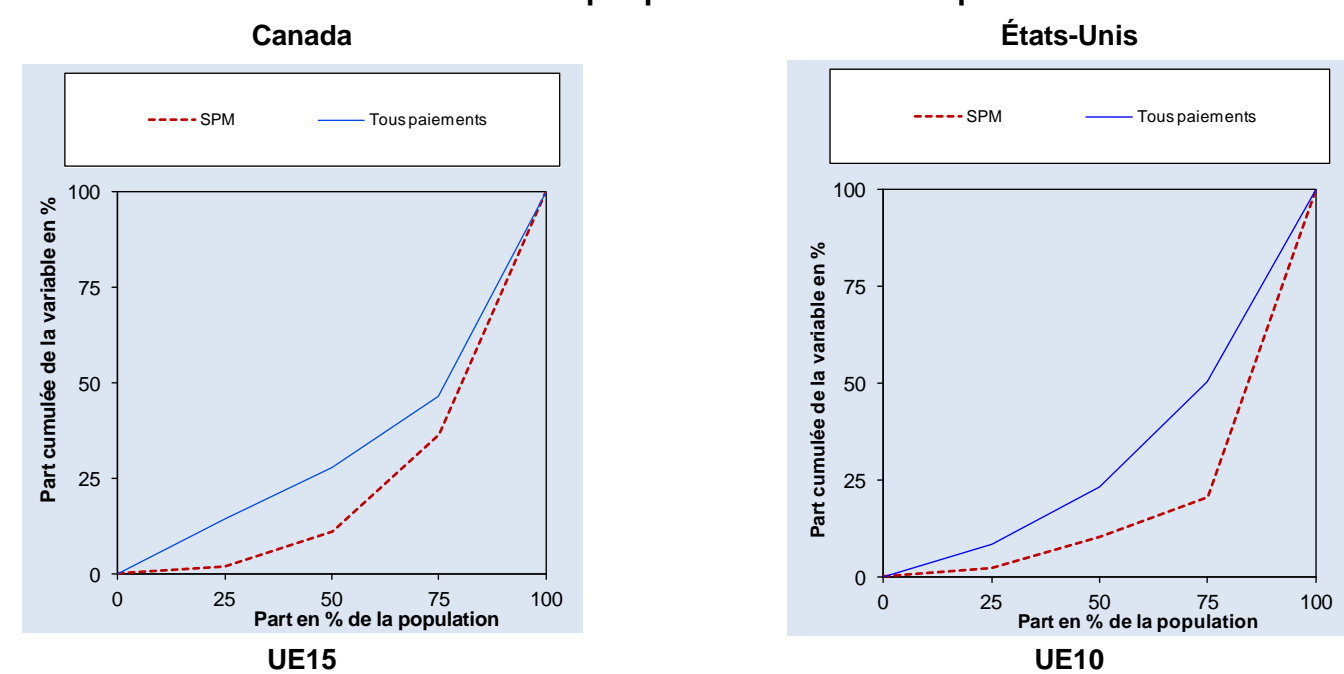

UE10
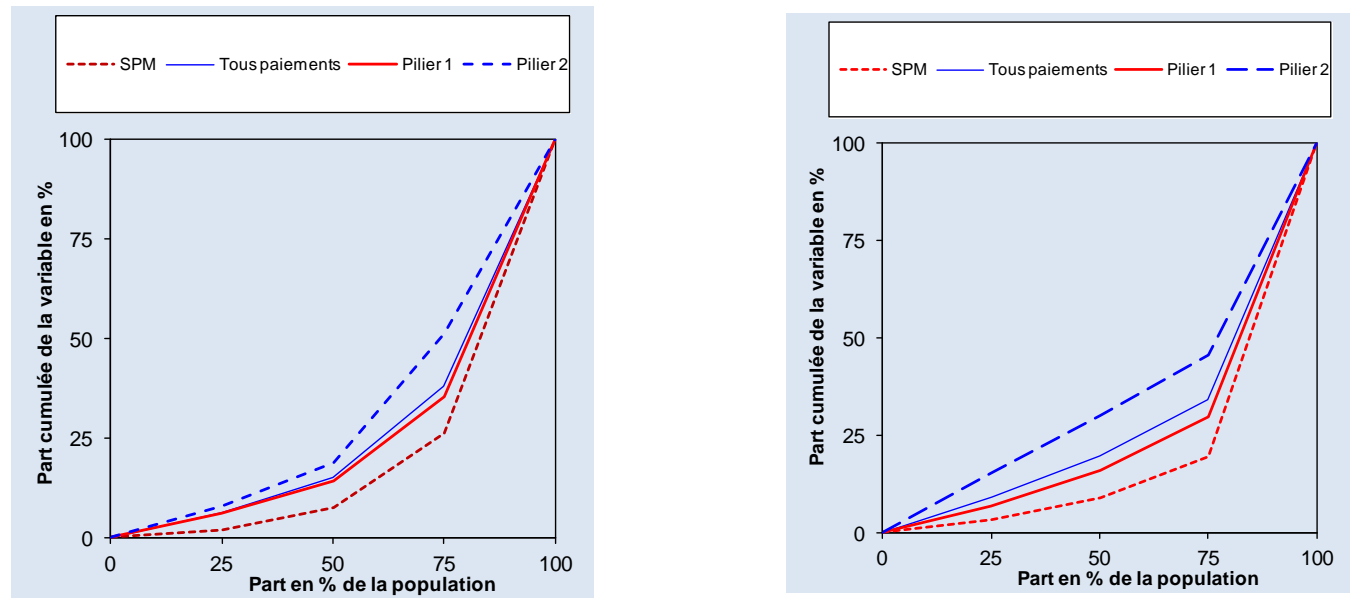

UE27

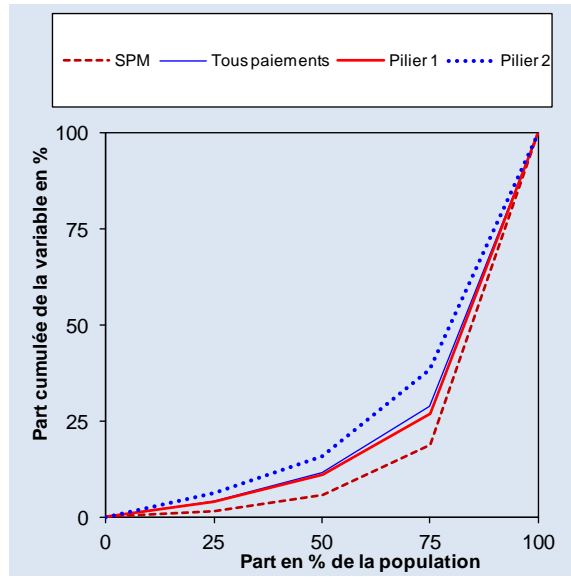

UE2

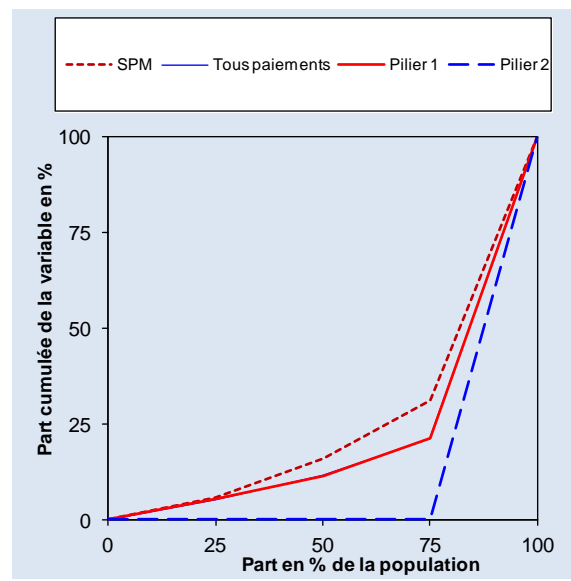



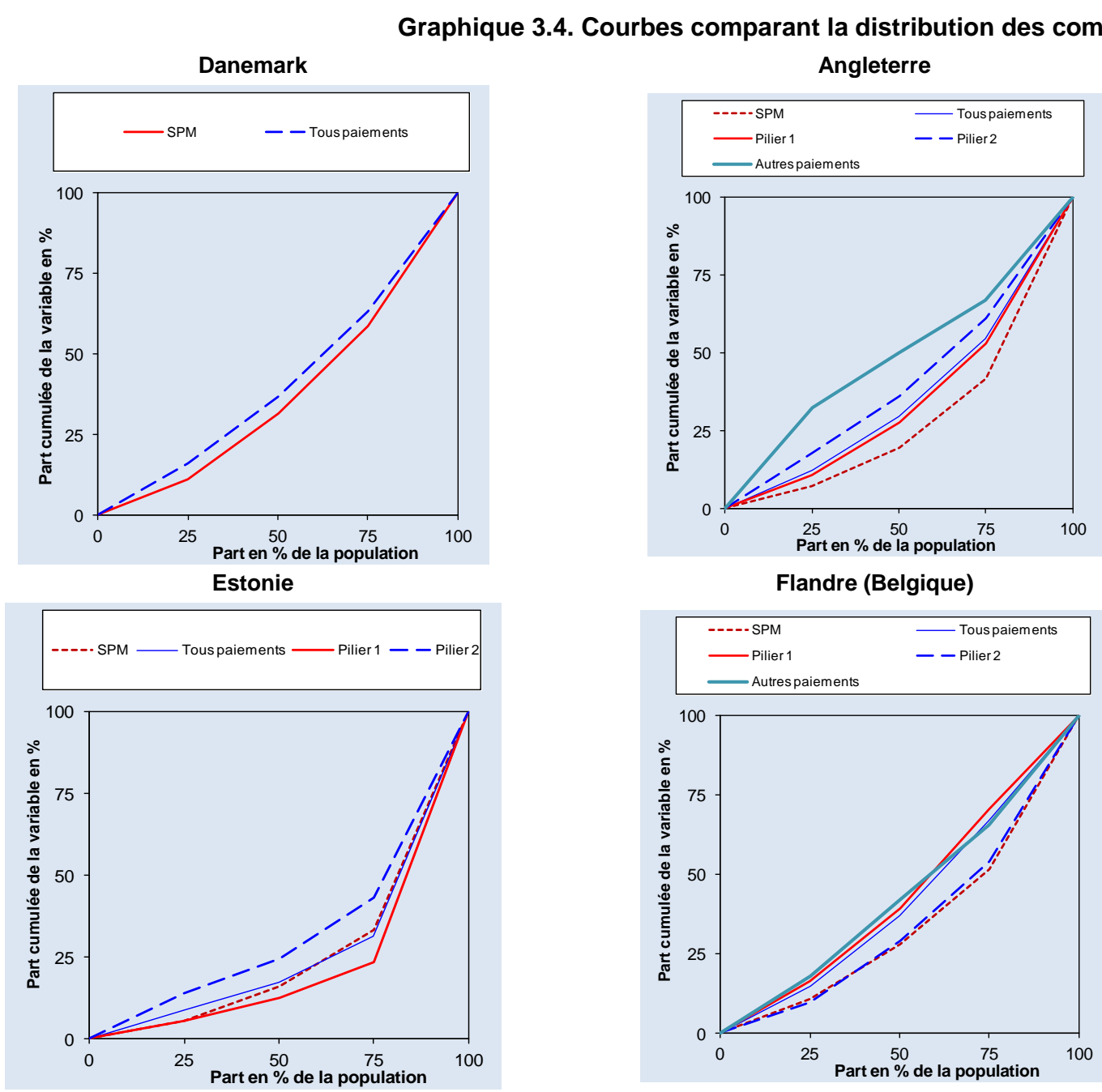

\section{mposantes du soutien, 2007 (suite)}
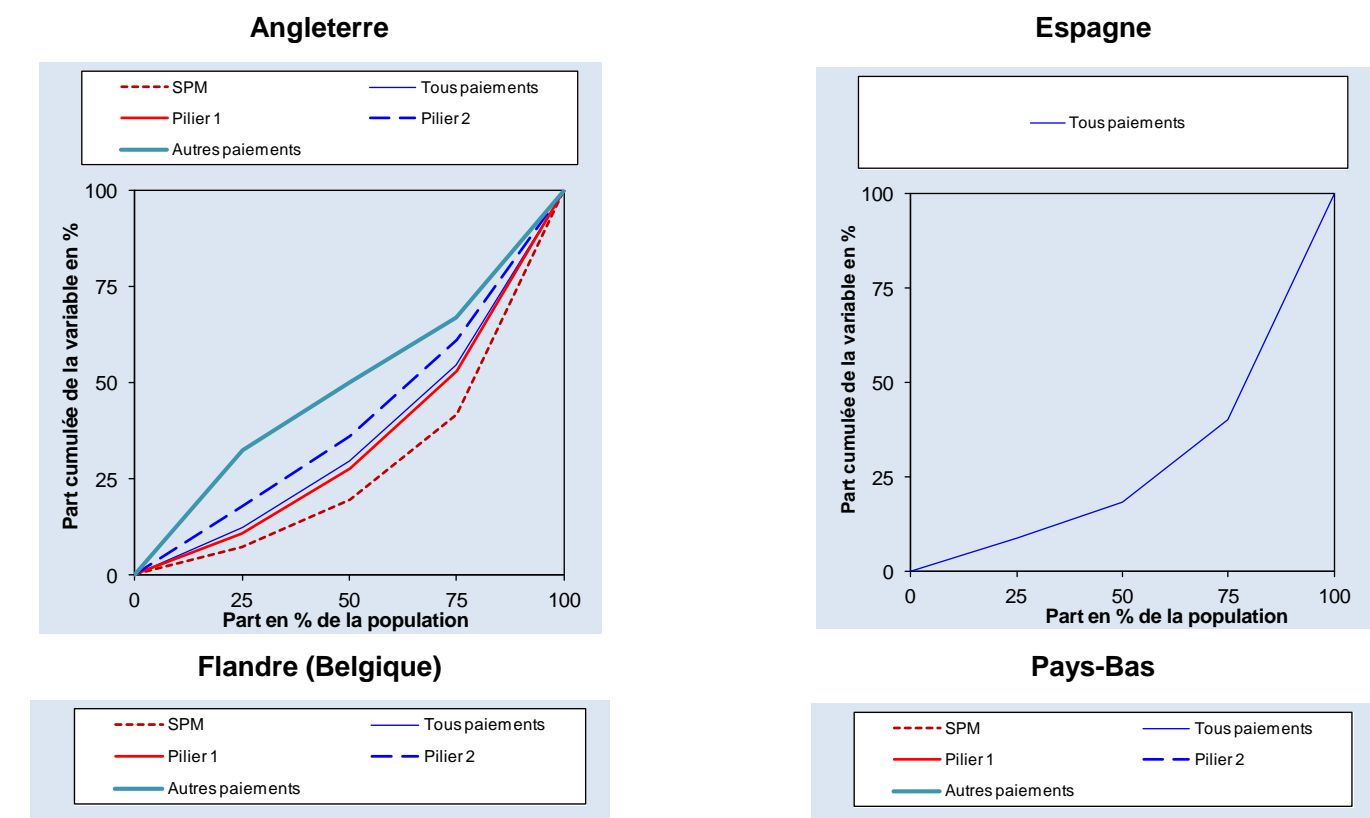

Pays-Bas
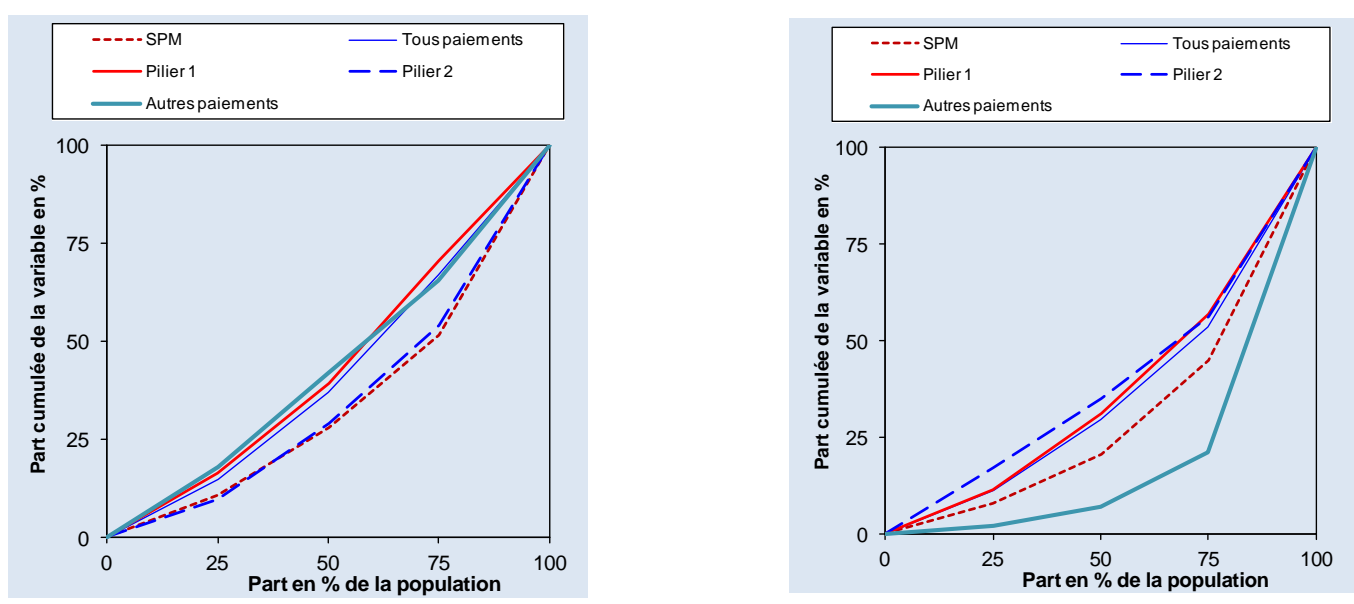
26 - DisTRIBUTION DU SOUTIEN ET DES REVENUS DANS L'AGRICULTURE

Graphique 3.4. Courbes comparant la distribution des composantes du soutien, 2007 (suite)

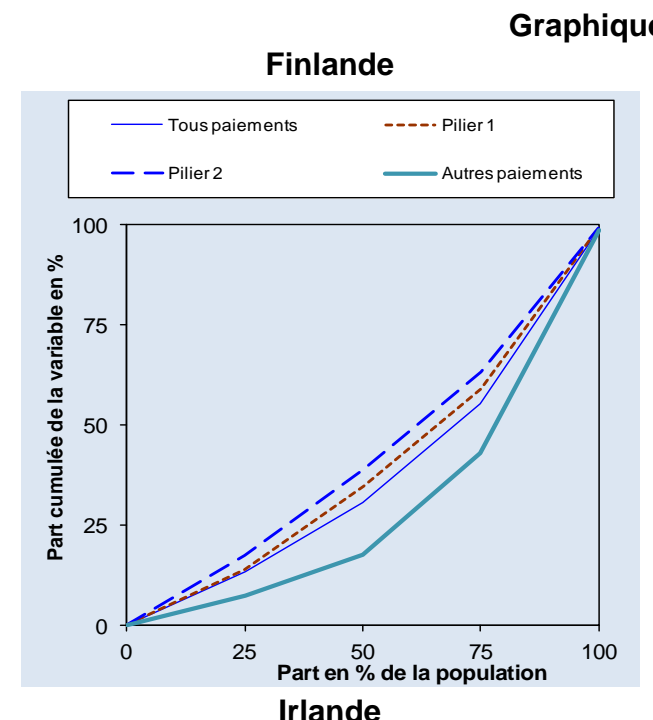

\section{France}

\begin{tabular}{|ll|}
\hline - Tous paiements & ---- - Pilier1 \\
- - Pilier 2 & Autres paiements \\
\hline
\end{tabular}

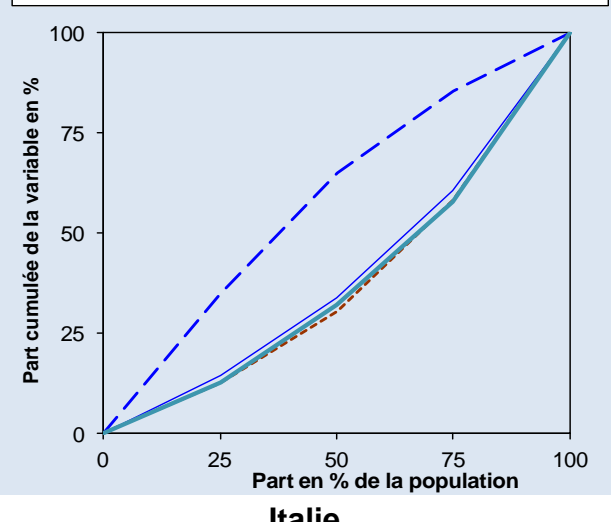

Italie
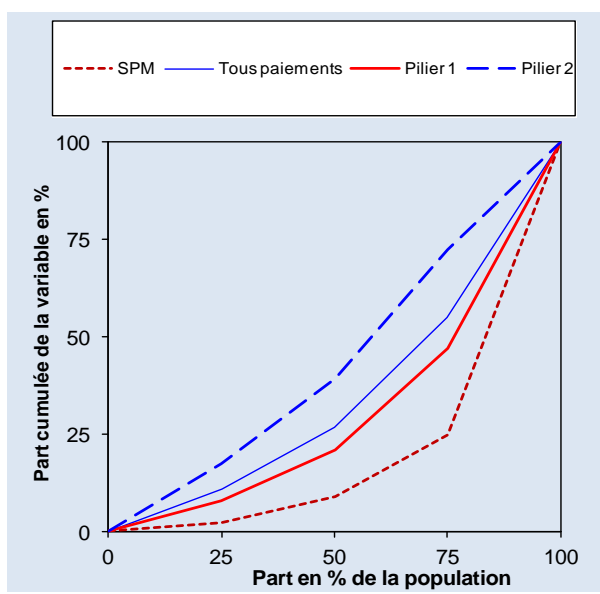
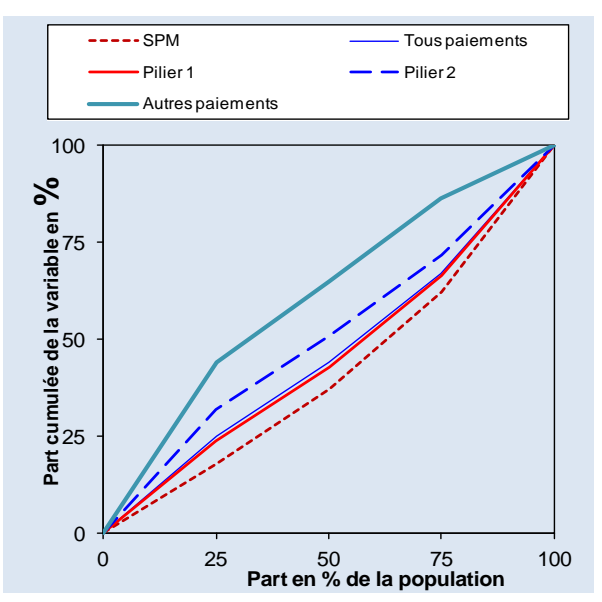

Allemagne

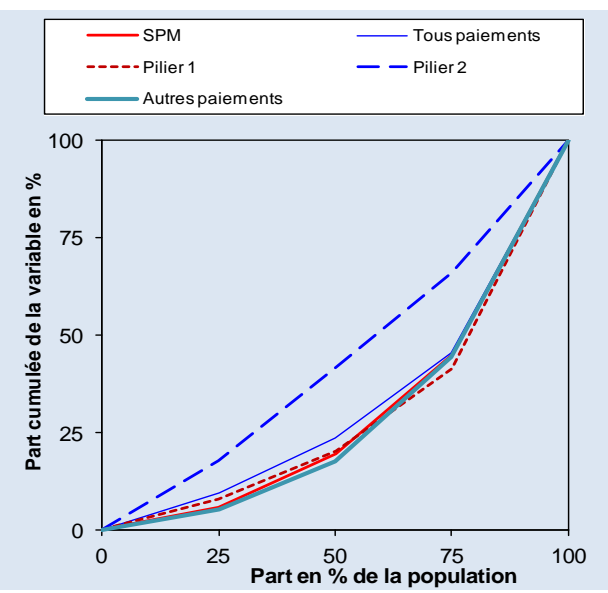

Les courbes sont réalisées sur la base des quartiles établis à partir de la production agricole brute (PAB), et non des données relatives aux différentes exploitations. Cela explique pourquoi certaines courbes sont convexes quand un quartile inférieur cumule plus de $25 \%$ d'une variable (paiements effectués en dehors du premier pilier dans le cas présent) ;

SPM : soutien des prix du marché ; population : exploitations agricoles représentées par l'échantillon. Les variables sont définies dans les notes du tableau C1 en annexe.

Source : tableaux transmis par les participants. 


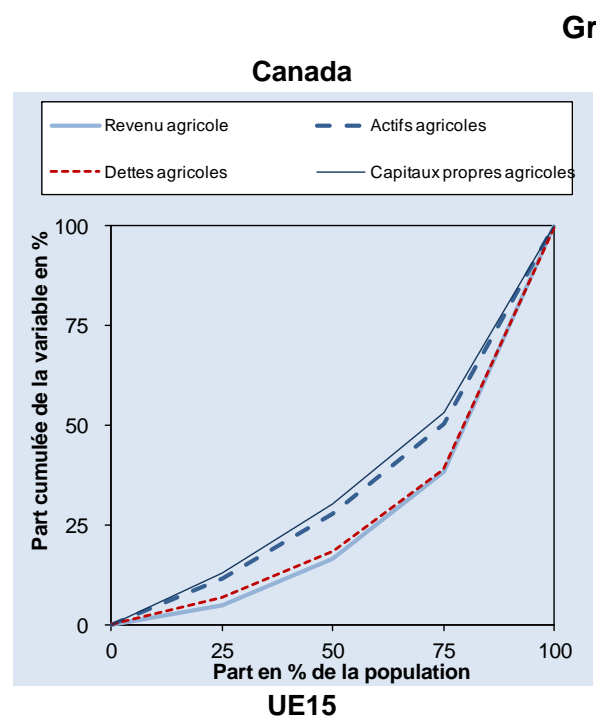

Graphique 3.5. Courbes comparant la distribution des revenus et des actifs, 2007

États-Unis
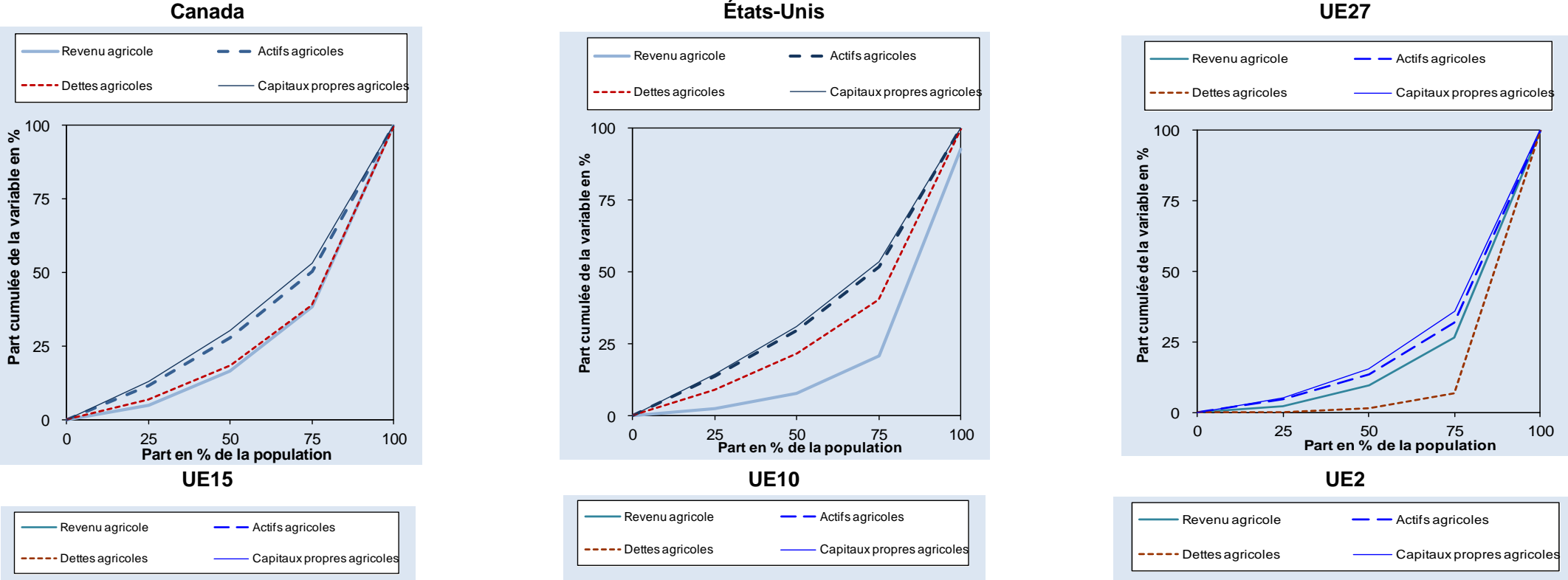

UE2
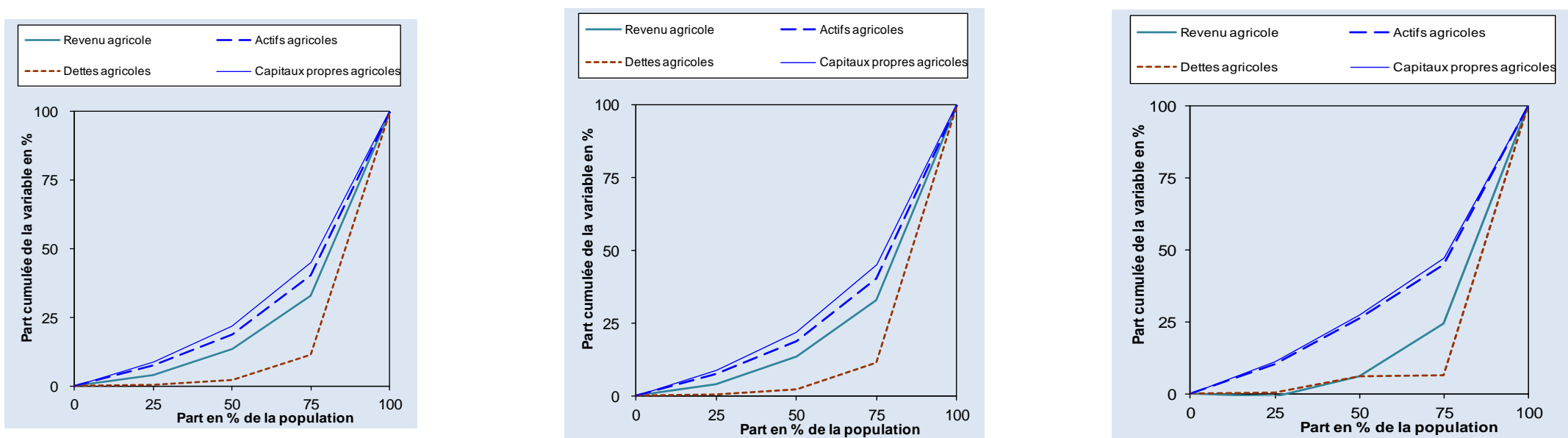
Danemark

Aucune donnée fournie.

\section{Estonie}

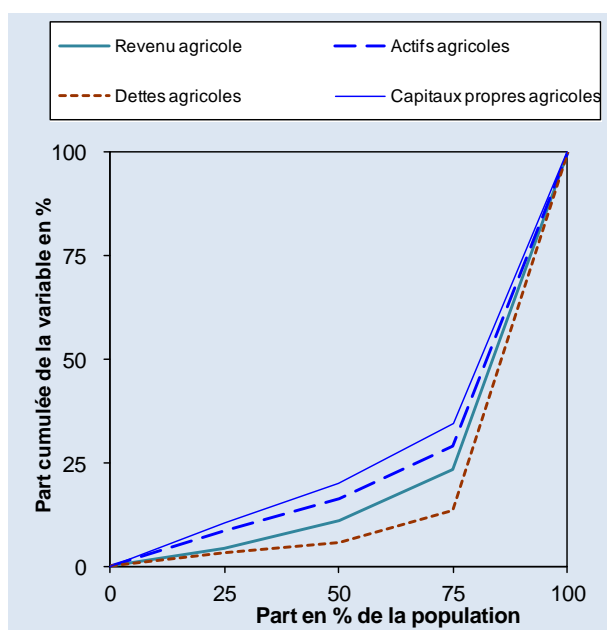

Graphique 3.5. Courbes comparant la distribution des revenus et des actifs, 2007 (suite)

Angleterre

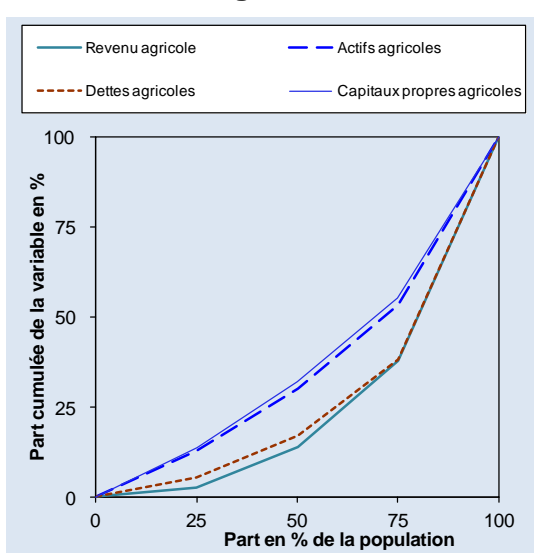

Flandre (Belgique)
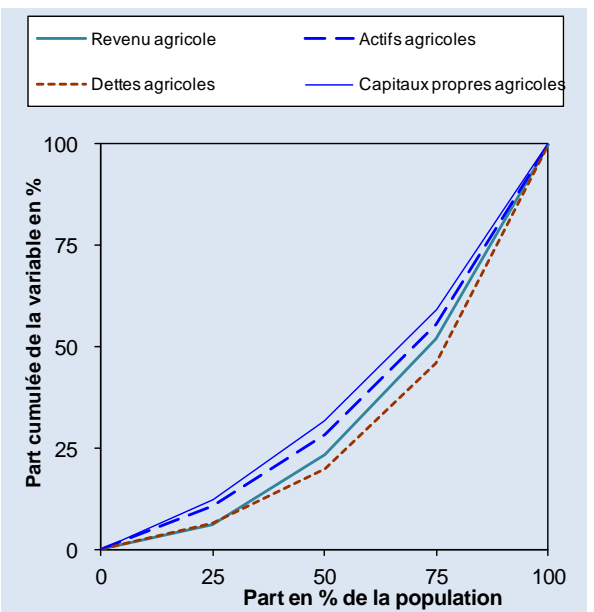

\section{Espagne}
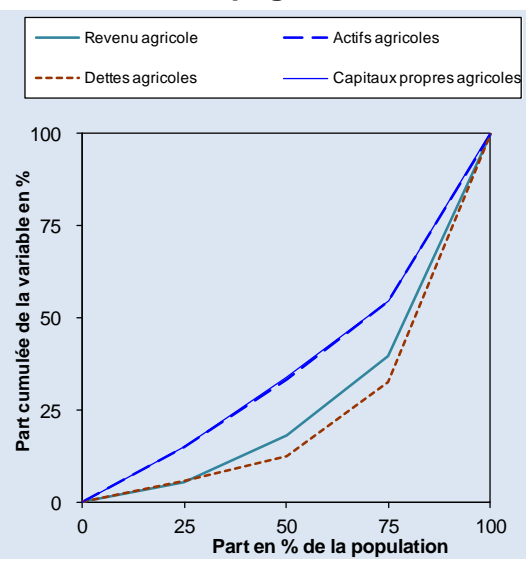

Pays-Bas
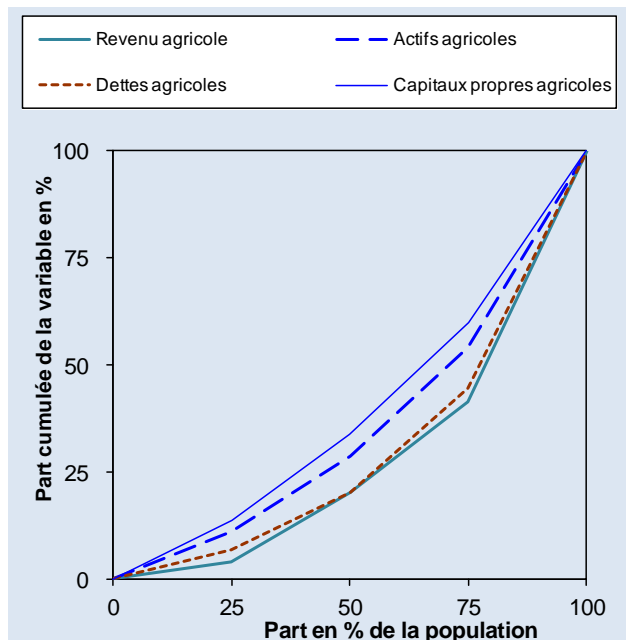


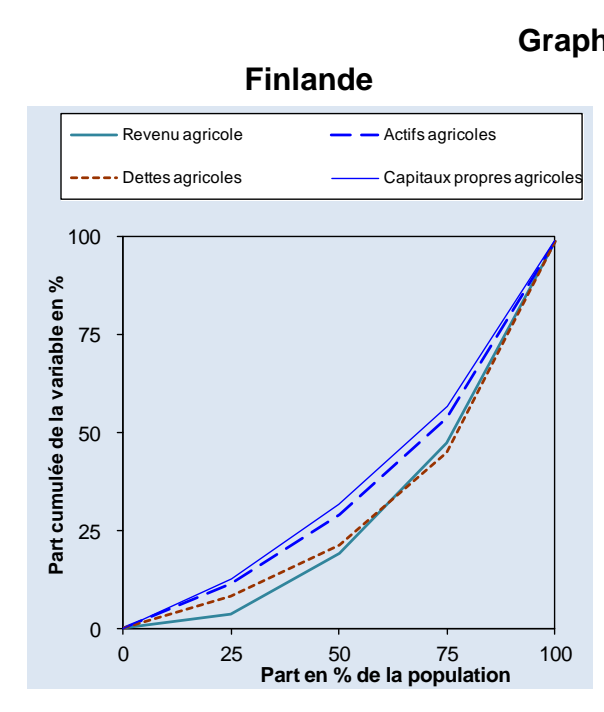

Graphique 3.5. Courbes comparant la distribution des revenus et des actifs, 2007 (suite)

France

\section{Allemagne}

Irlande

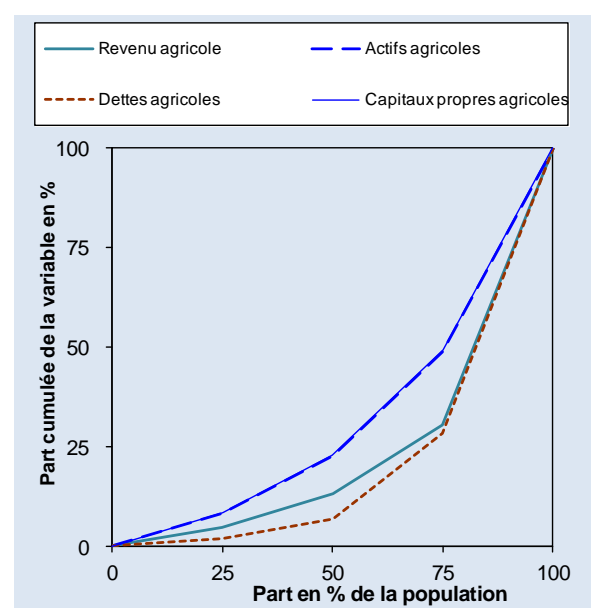

Aucune donnée fournie.

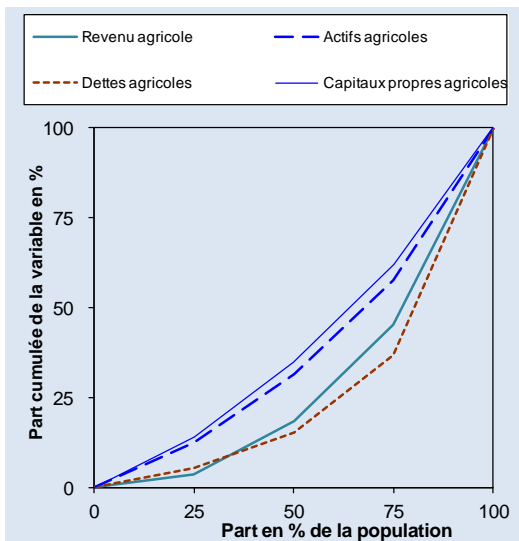

Italie

Les courbes sont réalisées sur la base des quartiles établis à partir de la production agricole brute (PAB); SPM : soutien des prix du marché ; population: exploitations agricoles représentées par l'échantillon.

Les variables sont définies dans les notes du tableau $\mathrm{C} 1$ en annexe.

Aucune donnée fournie.

Source : tableaux transmis par les participants. 


\section{Différences selon la taille d'exploitation}

Pour déterminer les différences entre exploitations, il est également possible de mesurer le ratio entre le niveau moyen du soutien et des autres composantes du revenu pour un même groupe d'exploitations (quartile, type d'exploitations, région), et le niveau moyen de ces variables pour l'ensemble des exploitations.

D'après le graphique 3.6, les $25 \%$ d'exploitations les plus grandes reçoivent, en moyenne, des niveaux d'aide qui sont deux fois supérieurs à la moyenne de l'ensemble des exploitations dans la plupart des pays, sauf au Danemark, en Flandre belge et en Italie, où les ratios sont d'environ $1.5^{12}$. Ce ratio est proche de trois dans la zone UE27. Le revenu agricole moyen des $25 \%$ d'exploitations les plus grandes est environ le double de celui de l'ensemble des exploitations, quand ce n'est pas plus : il est approximativement 2.5 fois plus élevé en Angleterre, au Canada, aux États-Unis et en Irlande, et trois fois supérieur dans les agrégats de l'Union européenne; l'écart est encore plus grand en Estonie. Cela étant, quand les données sont disponibles, le revenu moyen des ménages agricoles est plus proche du double de celui de l'ensemble des exploitations. Ces chiffres démontrent que les grandes exploitations présentent, en moyenne, des niveaux de revenu non agricole inférieurs à ceux des exploitations plus petites. Les mauvais résultats en termes de revenu des $25 \%$ plus grandes exploitations au Danemark sont liés aux grandes exploitations d'élevage porcin qui, en moyenne, ont des revenus négatifs en 2007 (tableau C5 en annexe). La situation était différente les années précédentes. En 2006, le rapport entre la moyenne des $25 \%$ plus grandes exploitations et la moyenne de l'ensemble des exploitations était de 1.6 pour le revenu agricole contre 0.7 en 2007 (tableau C3 en annexe).

En revanche, les $25 \%$ d'exploitations les plus petites reçoivent un soutien total moyen égal à moins de la moitié de celui de l'ensemble des exploitations, alors que leur taille moyenne est d'environ le quart de la moyenne générale, voire moins. La seule exception est l'Italie où le soutien moyen des $25 \%$ d'exploitations les plus petites est proche de celui de l'ensemble des exploitations. Le ratio entre le premier quartile et l'ensemble des exploitations est généralement plus faible pour la production agricole brute, les recettes et le revenu agricole que pour le soutien, et en particulier pour les paiements. Ainsi, bien que les exploitations plus petites bénéficient en moyenne d'aides moins importantes, celles-ci constituent, semble-t-il, une part plus grande de leurs recettes : environ deux tiers en Finlande et en Irlande, $50 \%$ en Estonie, $40 \%$ en Angleterre, $30 \%$ en France et dans la zone UE15, et $25 \%$ en Allemagne et dans la zone UE27 (graphique 3.7). La part importante des paiements en faveur des quartiles inférieurs en Estonie met en évidence la faible valeur des recettes venant du marché dans les exploitations plus petites. En Irlande, le phénomène est dû au grand nombre de petites exploitations d'élevage bovin tributaires des paiements, tandis qu'en Finlande la part des paiements dans les recettes brutes est élevée pour toutes les exploitations du fait des paiements nationaux, surtout dans les régions du Nord. Aux États-Unis, tous les quartiles affichent à peu près la même proportion de soutien dans les recettes agricoles (environ $8 \%$ ), mais les $25 \%$ d'exploitations les plus petites affichent un SPM inférieur et des paiements supérieurs à la moyenne, alors que pour les $25 \%$ d'exploitations les plus grandes, la situation est inverse. Le graphique 3.7 montre également combien la part du soutien dans les recettes agricoles brutes varie selon le pays, quelque soit la taille des exploitations.

12. Bien qu'il soit inclus dans la production agricole brute, le SPM n'est pas indiqué séparément pour l'Espagne, la Finlande et la France. Le soutien total ne peut donc pas être calculé, et l'examen de ces pays se limite à la distribution des paiements. 
Graphique 3.6. Différences selon la taille d'exploitation, 2007

Ratio de la moyenne du quartile sur la moyenne de l'ensemble des exploitations

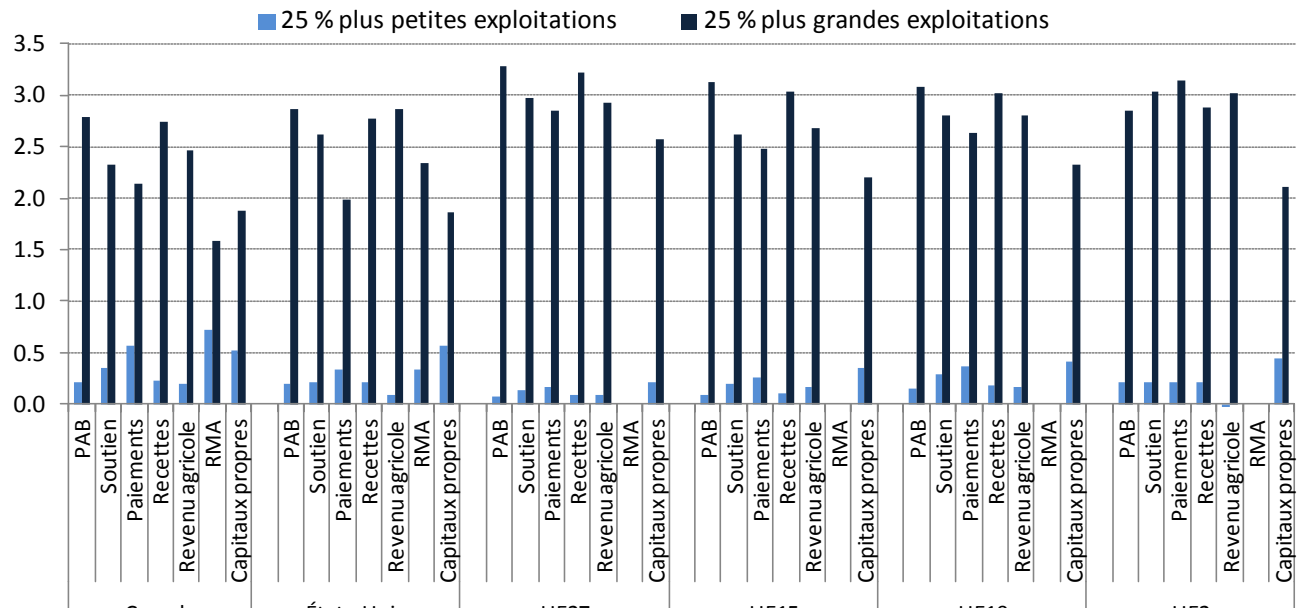

États-Unis

UE27

UE15

UE10

UE2
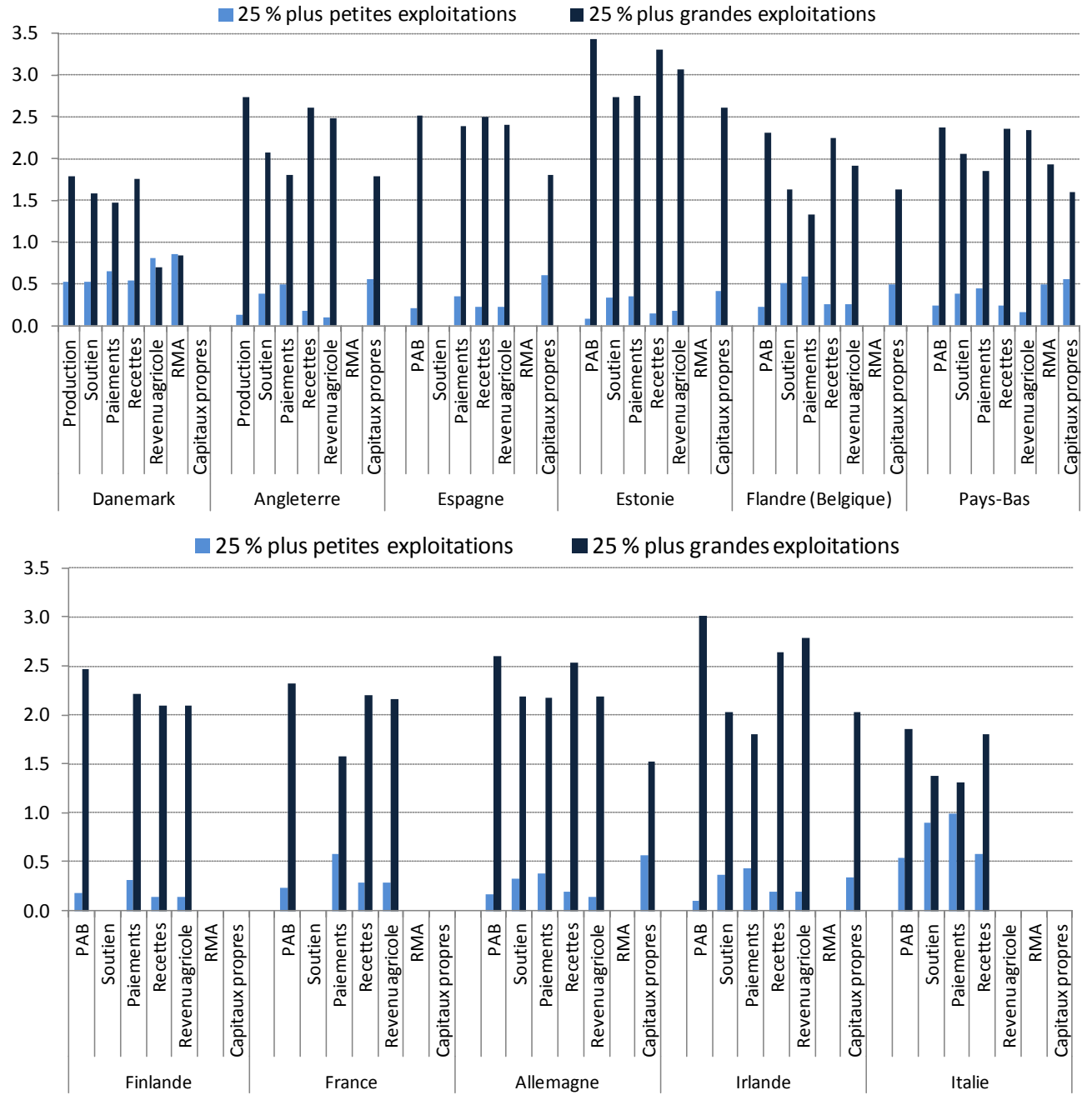

Pour la Finlande et la France, les données relatives au SPM ne sont pas disponibles. En Italie, le revenu agricole n'est pas inclus en raison de la présence de nombres négatifs. Les quartiles sont établis à partir de la production agricole brute (voir notes du tableau C1 en annexe). $\mathrm{PAB}$ : production agricole brute ; RMA : revenu des ménages agricoles. Source : tableau C3 en annexe. 
Graphique 3.7. Part du soutien dans les recettes agricoles brutes, par taille d'exploitation, 2007

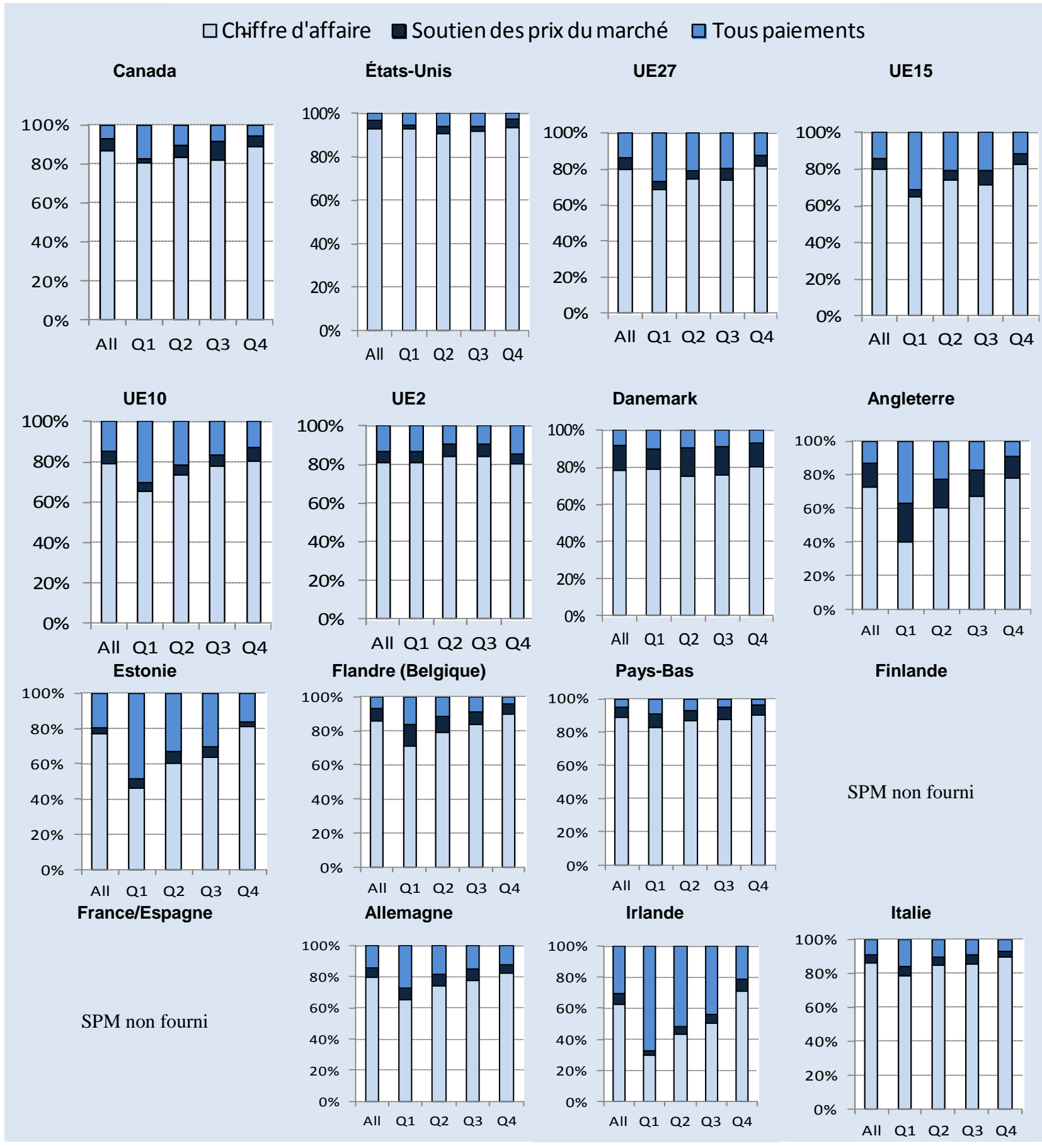

All: Ensemble des exploitations; Q1, Q2, Q3, Q4 : quartiles établis à partir de la production agricole brute. Q1 : les $25 \%$ d'exploitations les plus petites ; Q4 : les $25 \%$ d'exploitations les plus grandes. Les pays pour lesquels le SPM n'est pas disponible ne sont pas inclus dans ce graphique.

Source : tableau C4 en annexe.

\section{Distribution du soutien et des revenus par type d'exploitations}

La distribution du soutien par type d'exploitations varie d'un pays à l'autre. Elle dépend aussi du type de soutien. Dans la plupart des pays, le soutien total et les paiements ne se distribuent pas de la même façon selon le type d'exploitations. Au Canada et aux États-Unis, les exploitations laitières, qui représentent environ $15 \%$ de l'ensemble des 
exploitations et de la production agricole brute, concentrent approximativement $85 \%$ du soutien des prix du marché et $5 \%$ des paiements. Au Canada, les exploitations de production végétale comptent environ pour $40 \%$ de l'ensemble des exploitations et pour $25 \%$ de la production agricole brute, et absorbent approximativement $40 \%$ des paiements et moins de $1 \%$ du soutien des prix du marché (graphique 3.8). Aux États-Unis, ce type d'exploitations regroupe la moitié des unités et perçoit près des trois quarts des paiements.

Le soutien est également concentré sur les exploitations laitières dans la plupart des dix pays de l'Union européenne inclus dans cette analyse: hormis en Italie, ces exploitations reçoivent entre un quart et deux tiers du soutien total $(22 \%$ en moyenne dans la zone UE27) et de 30 à $75 \%$ du SPM (dans la zone UE27 toutefois, elles reçoivent en moyenne $24 \%$ du SPM). La concentration du soutien dans les exploitations laitières est particulièrement prononcée au Danemark car cette catégorie comprend une importante production de viande bovine et reçoit le soutien correspondant. Les exploitations de grandes cultures reçoivent entre un quart et un tiers du soutien et un tiers des paiements en Allemagne, en Estonie, en Finlande et en Italie, mais moins de $15 \%$ du soutien dans les autres États membres de l'Union européenne inclus, où les exploitations d'élevage sont plus importantes. Dans la zone EU27, elles bénéficient de $37 \%$ de l'ensemble des paiements et de $34 \%$ du soutien total.

Aux Pays-Bas, les exploitations de production végétale et d'élevage bovin représentent dans un cas comme dans l'autre $15 \%$ de l'ensemble des exploitations et reçoivent une part correspondante du soutien et une proportion légèrement supérieure des paiements $(20 \%)$. En revanche, les pépinières et les exploitations de floriculture, qui constituent $13 \%$ des exploitations, perçoivent seulement $5 \%$ des paiements et $9 \%$ du soutien total ${ }^{13}$. En Angleterre, la moitié des paiements va aux exploitations mixtes («Autres »), qui représentent $38 \%$ des exploitations et bénéficient du même pourcentage du soutien total. Cette proportion est également élevée (plus d'un quart du soutien) en Allemagne, en Estonie, en Flandre belge, en France et en Italie.

En Estonie et en Allemagne, la distribution du soutien total ne diffère guère de celle des paiements. En Allemagne, les exploitations de production végétale absorbent $25 \%$ du soutien et $29 \%$ des paiements, tandis que les exploitations mixtes (autres), soit environ un quart des exploitations, bénéficient de $30 \%$ du soutien et des paiements. En Estonie, les exploitations de grandes cultures perçoivent environ un tiers du soutien total et des paiements, et les exploitations mixtes, approximativement un quart de ces deux variables. Le pourcentage de soutien dont bénéficie chaque type d'exploitations est également très proche de la part des exploitations de ce type dans l'ensemble des exploitations couvertes. En Finlande, les élevages perçoivent proportionnellement plus de paiements que les exploitations de production végétale, mais les paiements du premier pilier sont distribués en fonction du nombre d'exploitations au sein de la population considérée. La part élevée du soutien, en particulier du SPM, que reçoivent les élevages bovins en Flandre belge peut s'expliquer par l'importance de la production laitière dans les exploitations d'élevage mixte. En France, les exploitations de production végétale et d'élevage de bovins à l'herbe obtiennent une proportion de l'ensemble des paiements supérieure à la part qu'elles représentent au sein de la population considérée, tandis qu'en Irlande on constate le même phénomène pour les exploitations laitières et les exploitations d'élevage bovin, ovin et caprin.

13. On suppose que le taux du SPM pour l'horticulture est égal à celui appliqué à la moyenne de toutes les cultures. 
Graphique 3.8. Distribution du soutien par type d'exploitations, 2007

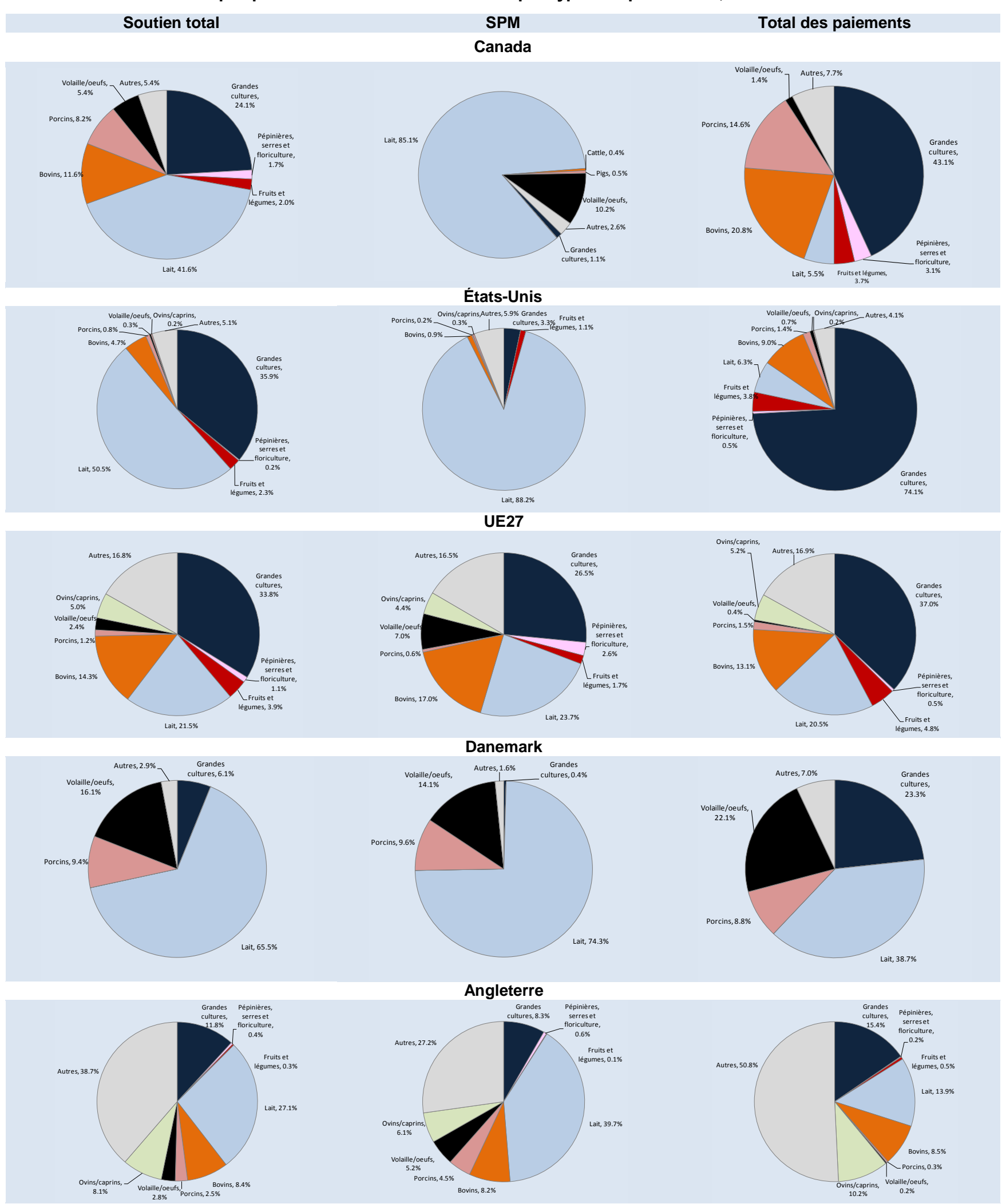


Graphique 3.8. Distribution du soutien par type d'exploitations, 2007 (suite)

Soutien total
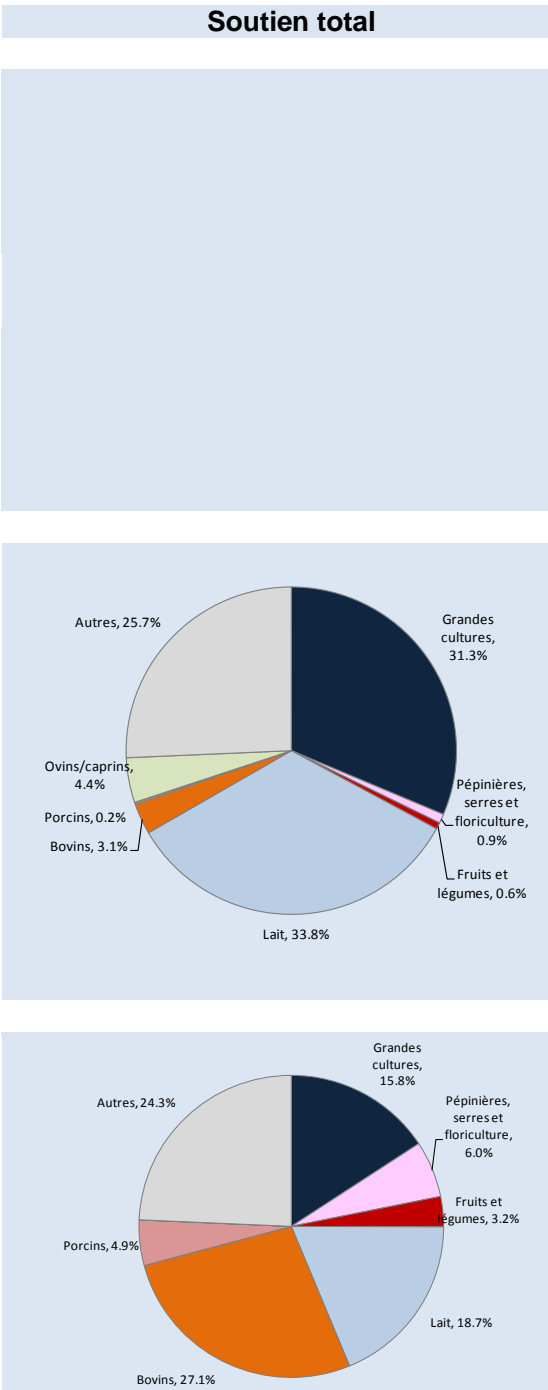

Bovins, $27.1 \%$

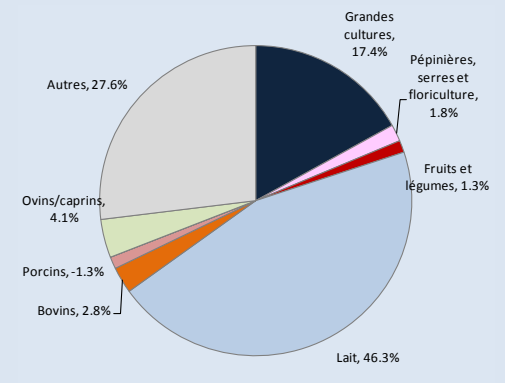

Flandre (Belgique)

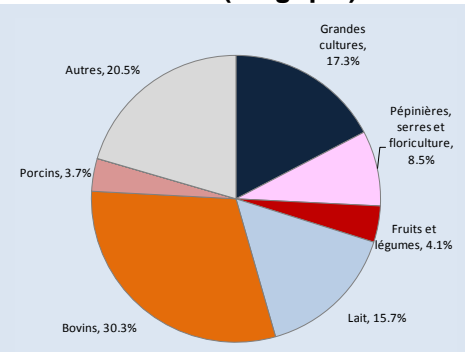

Pays-Bas
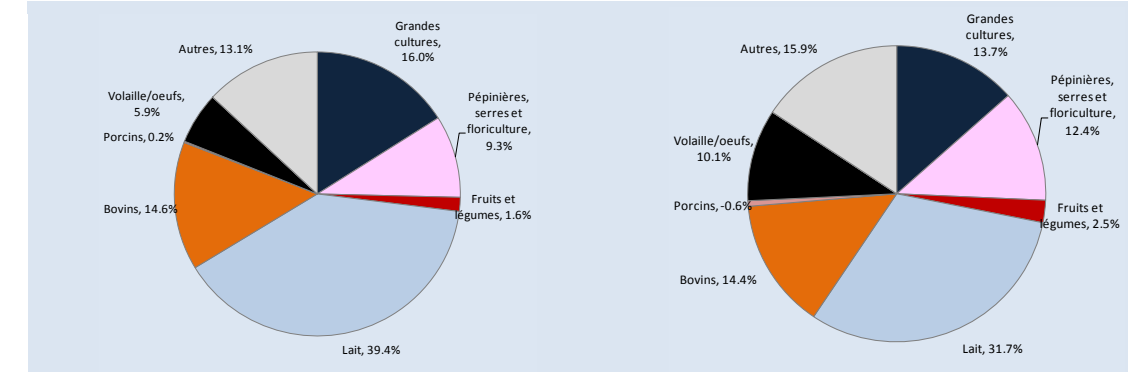

SPM

Espagne

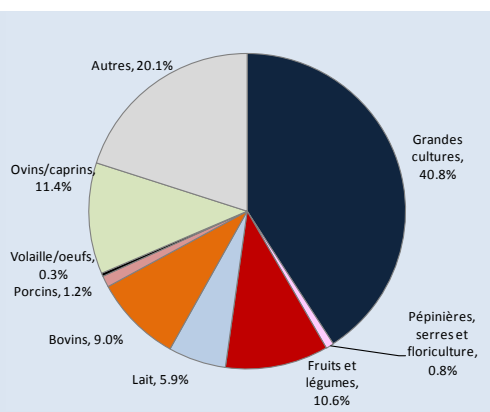

Total des paiements
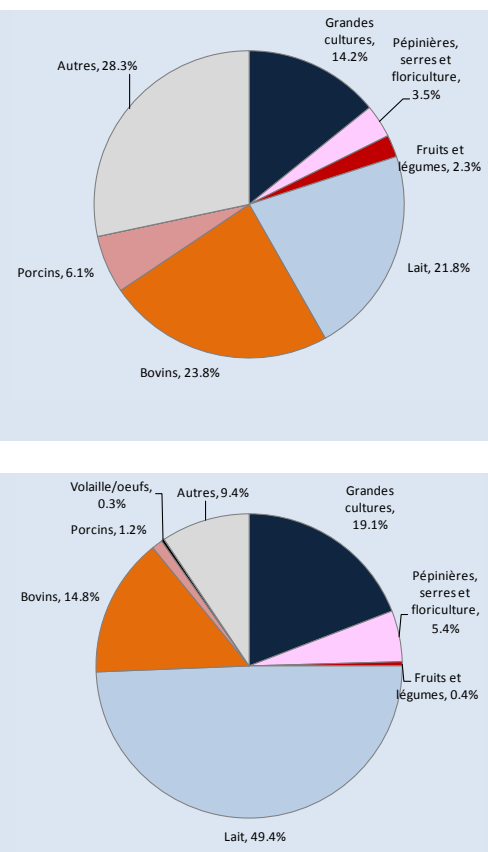


\section{France}

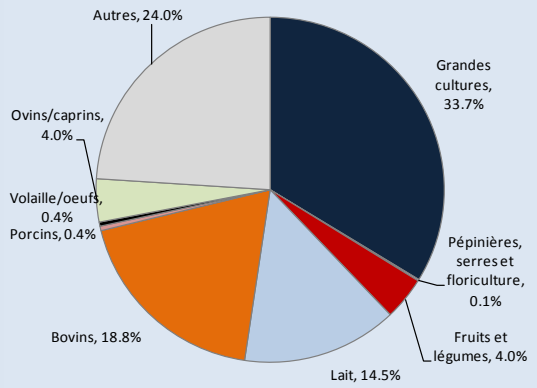

Finlande

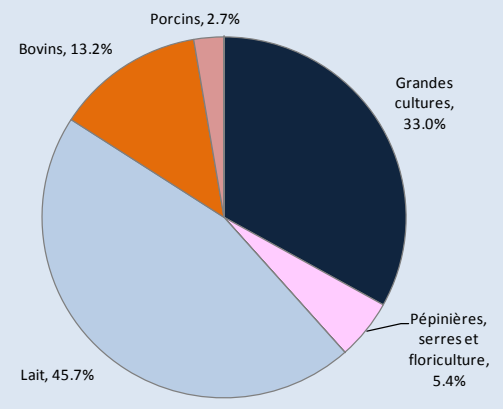

Allemagne

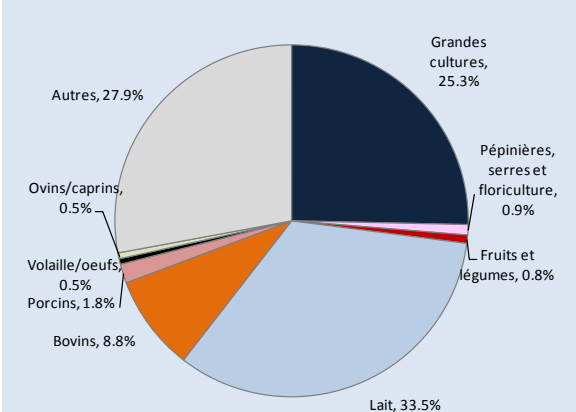

Lait, 33.5\%
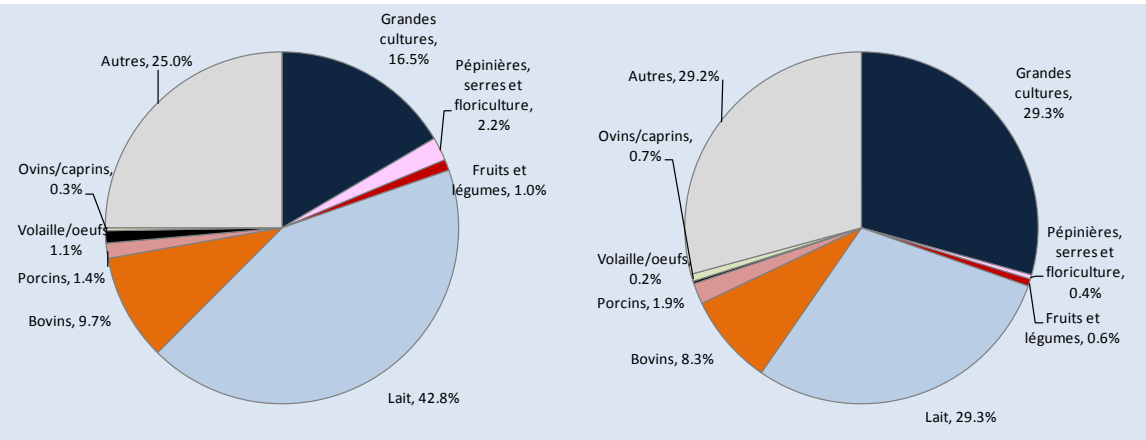

Irlande
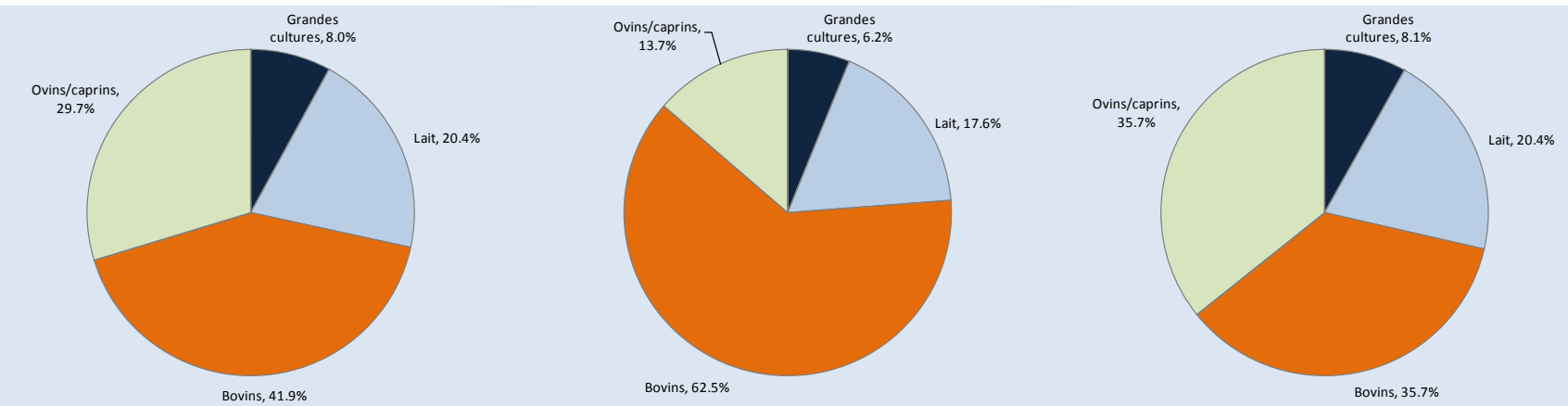


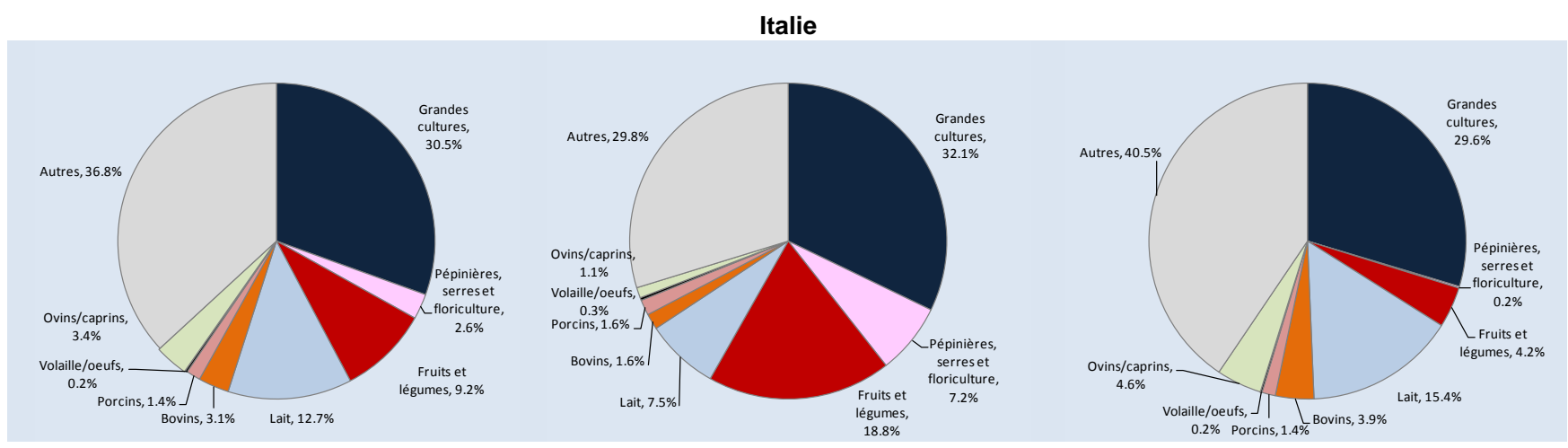

Source : tableau C5 en annexe.

\section{Différences selon le type d'exploitations}

La comparaison de la moyenne des exploitations d'un type donné avec celle de l'ensemble des exploitations fait apparaître des différences importantes d'un type à l'autre en termes de revenu moyen et de soutien, et les écarts constatés sont parfois plus grands que ceux liés à la taille des exploitations. Les ratios entre la moyenne de chaque type d'exploitations et celle de l'ensemble des exploitations sont illustrés au graphique 3.9 pour 2007. Une grande partie du soutien est contracyclique (le SPM, mais aussi les paiements au Canada), ce qui joue notamment sur les résultats obtenus par type d'exploitations. En conséquence, on se référera aux ratios de 2004, de 2006 et de 2007, présentés au tableau C5 en annexe, selon que de besoin.

Si le revenu moyen des exploitations de grandes cultures au Canada est sensiblement identique à celui de l'ensemble des exploitations, leur chiffre d'affaires brut est, en revanche, inférieur. En 2007, elles ont reçu grosso modo le même paiement moyen que l'ensemble des exploitations, mais moins de soutien (graphique 3.9). Cette différence entre le soutien et les paiements est due au fait que les exploitations laitières canadiennes bénéficient de l'équivalent de trois fois le soutien moyen de l'ensemble des exploitations, principalement sous forme de soutien des prix du marché, bien que, au vu de leur production agricole brute moyenne, elles soient légèrement plus petites au Canada (graphique 3.9 et tableau C5 en annexe). Cette situation est relativement stable sur les trois années examinées. Au Canada, les exploitations d'élevage porcin et avicole ont une taille deux fois supérieure à la moyenne, et, en 2007, elles ont également absorbé un soutien total moyen supérieur à celui de l'ensemble des exploitations. Ce dernier chiffre concerne 2007 uniquement ; le pourcentage est bien moins élevé les années précédentes ${ }^{14}$. Le pic de 2007 traduit la nature contracyclique du soutien canadien, qui repose essentiellement sur la fourniture de filets de sécurité aux agriculteurs. Les hauts niveaux d'aide accordés en 2007 ont contribué à accroître le revenu moyen des exploitations avicoles : ce dernier a atteint trois fois celui de l'ensemble des exploitations, alors que celui des exploitations d'élevage porcin est resté bien en deçà de la moyenne, et ce pour la deuxième année consécutive. En termes de taille et de revenu, les exploitations d'élevage bovin sont dans la moyenne de l'ensemble des exploitations mais elles reçoivent

14. Il convient de noter que les paiements peuvent être perçus plusieurs mois après la vente des produits. Ils peuvent même être enregistrés sur l'exercice suivant dans les comptes des exploitations. 
moins de soutien. En terme de capital propre, les exploitations de grandes cultures sont dans la moyenne et les exploitations d'élevage bovin et porcin en deçà.

Aux États-Unis, les exploitations de grandes cultures ont un revenu inférieur à celui de la moyenne des exploitations mais proportionnel à leur taille. Les exploitations laitières sont de taille supérieure à la moyenne de l'ensemble des exploitations, reçoivent d'avantage de soutien, principalement sous la forme de SPM, mais leur revenu agricole est proche de la moyenne. Les exploitations d'élevage bovin sont de taille moyenne mais leur revenu représente la moitié de celui de la moyenne de l'ensemble des exploitations. Cependant, les éleveurs bovins ont davantage de revenus non agricoles que les éleveurs laitiers. Les élevages porcins ont une taille près de deux fois supérieure à la moyenne et leur revenu moyen est également deux fois plus élevé bien qu'ils ne reçoivent que peu d'aides. En revanche, les exploitations avicoles sont nettement plus petites et affichent un revenu sensiblement plus faible.

Dans les États membres de l'Union européenne, on trouve de très grandes exploitations dans le secteur avicole, lequel représente un faible pourcentage de l'ensemble des exploitations (graphique 3.9, tableau C5 en annexe). Les élevages avicoles reçoivent plus de soutien que la moyenne (quatre fois plus dans la zone UE27), mais leur taille est relativement plus grande encore. Les exploitations d'élevage porcin sont également plus grandes que la moyenne, mais à des degrés divers (entre 1.5 et 7 fois plus grandes selon les pays ; 2.7 fois plus grandes dans la zone UE27). En outre, bien que leur revenu ait été faible, voire négatif, en 2007, en raison du bas niveau des prix mondiaux, elles n'ont généralement pas bénéficié de soutien compensatoire. Au Danemark et aux Pays-Bas par exemple, les exploitations d'élevage porcin ont affiché en moyenne un revenu agricole négatif, et bien que le revenu non agricole ait atténué ces pertes, il ne les a pas compensées entièrement. Le revenu agricole des élevages porcins a également été négatif en Estonie en 2007, et très faible dans d'autres pays. Cela n'avait pas été le cas les années précédentes, au cours desquelles ce type d'exploitations avait enregistré un revenu nettement supérieur à la moyenne (jusqu'à trois fois plus élevé en Estonie en 2006).

Dans certains États membres de l'Union européenne inclus dans l'analyse ainsi que dans la zone UE27, les exploitations de grandes cultures ont une taille moyenne inférieure à celle de l'ensemble des exploitations, mais leur taille économique est plus grande en Angleterre, en France et en Irlande (graphique 3.9). Elles reçoivent proportionnellement plus de paiements du premier pilier et, à l'exception de l'Angleterre, un soutien total plus important (tableau C5 en annexe). Résultat: leur revenu rapporté à la moyenne de l'ensemble des exploitations est supérieur à leur taille relative.

Les exploitations laitières de la zone UE27 sont plus grandes que la moyenne et reçoivent davantage de soutien et de paiements. En revanche, les exploitations d'élevage bovin sont bien plus petites que la moyenne et bénéficient d'un soutien et de paiements plus élevés. Ces deux types d'exploitations affichent une taille moyenne inférieure à celle de l'ensemble des exploitations en Allemagne, en Flandre belge, en France et aux Pays-Bas, et obtiennent plus d'aides proportionnellement. Dans d'autres pays de l'Union européenne examinés, les élevages bovins sont également plus petits que la moyenne, mais les exploitations laitières sont plus grandes et enregistrent un revenu supérieur à la moyenne de l'ensemble des exploitations. De façon générale, les exploitations laitières reçoivent un soutien total plus élevé que la moyenne et leur revenu est nettement supérieur dans bien des cas (deux à trois fois plus élevé au Danemark et en Irlande), alors que les élevages bovins affichent des revenus sensiblement inférieurs à la moyenne, bien qu'ils bénéficient de niveaux de soutien non négligeables. Si l'on compare les types d'exploitations, c'est chez les éleveurs de bovins que la part du soutien dans les recettes brutes est la plus élevée, exception faite de l'Allemagne, où les élevages ovins et caprins 
dépendent davantage des aides (graphique 3.10 et tableau C6 en annexe). Cette proportion était importante quoique inférieure pour les exploitations laitières en $2007 \mathrm{du}$ fait de prix relativement élevés sur le marché mondial, mais elle était supérieure en 2004, avant l'application de la réforme de la Politique agricole commune de 2003.

Dans un grand nombre d'États membres de l'Union européenne, un pourcentage relativement élevé d'exploitations (un quart en Allemagne et en Estonie, et $38 \%$ en Angleterre) se classent dans la catégorie «Autres». Cet agrégat, qui réunit les exploitations mixtes, affiche des chiffres légèrement supérieurs à ceux de la moyenne des exploitations en termes de taille, de niveau de soutien et de revenu agricole. La part des «autres» exploitations est beaucoup plus modeste au Canada (8\%), aux États-Unis $(5 \%)$ et aux Pays-Bas (12\%).

Dans les États membres qui communiquent des données sur le revenu non agricole, les écarts entre le revenu agricole moyen et le revenu total des ménages agricoles ne sont pas importants, comme c'est le cas au Canada et aux États-Unis, car les enquêtent capturent plus particulièrement les exploitants à titre principal.

L'effet du soutien sur les écarts de revenu par type d'exploitations reste à déterminer. Au Canada, le soutien ne semble pas avoir d'incidence majeure sur les écarts observés entre types d'exploitations en termes de recettes agricoles, comparé aux écarts enregistrés en termes de production agricole brute. Il les réduit légèrement en Allemagne, en Angleterre, aux Pays-Bas et aux États-Unis, et plus nettement en Estonie et en Irlande. Les différences entre types d'exploitations ne semblent pas aussi marquées sur les capitaux propres que sur le revenu.

Le graphique 3.10 illustre les variations par type d'exploitations de la part du soutien dans les recettes agricoles brutes, variations qui coïncident avec celles observées par produit de base (OCDE, 2009b). La part du soutien dans les recettes agricoles brutes correspond également à l'importance relative de celui-ci dans le revenu. Au Canada et aux États-Unis, les exploitations laitières dérivent du SPM une part de leurs recettes supérieure à celle d'autres exploitations, tandis que dans l'agrégat de l'Union européenne et dans les États membres représentés, ce sont les exploitations d'élevage bovin et ovin qui sont les plus tributaires du soutien, que ce soit sous forme de SPM ou de paiements. Pour chaque type d'exploitations, la part du soutien dans les recettes brutes est généralement supérieure dans les États membres de l'Union européenne à ce qu'elle est en Amérique du Nord. Ce constat confirme les chiffres obtenus au niveau national : en 2007, les ESP atteignaient $19 \%$ au Canada, $10 \%$ aux États-Unis et $25 \%$ dans l'Union européenne. 
Graphique 3.9. Différences selon le type d'exploitations, 2007

Ratio de la moyenne des différents types d'exploitations sur celle de l'ensemble des exploitations

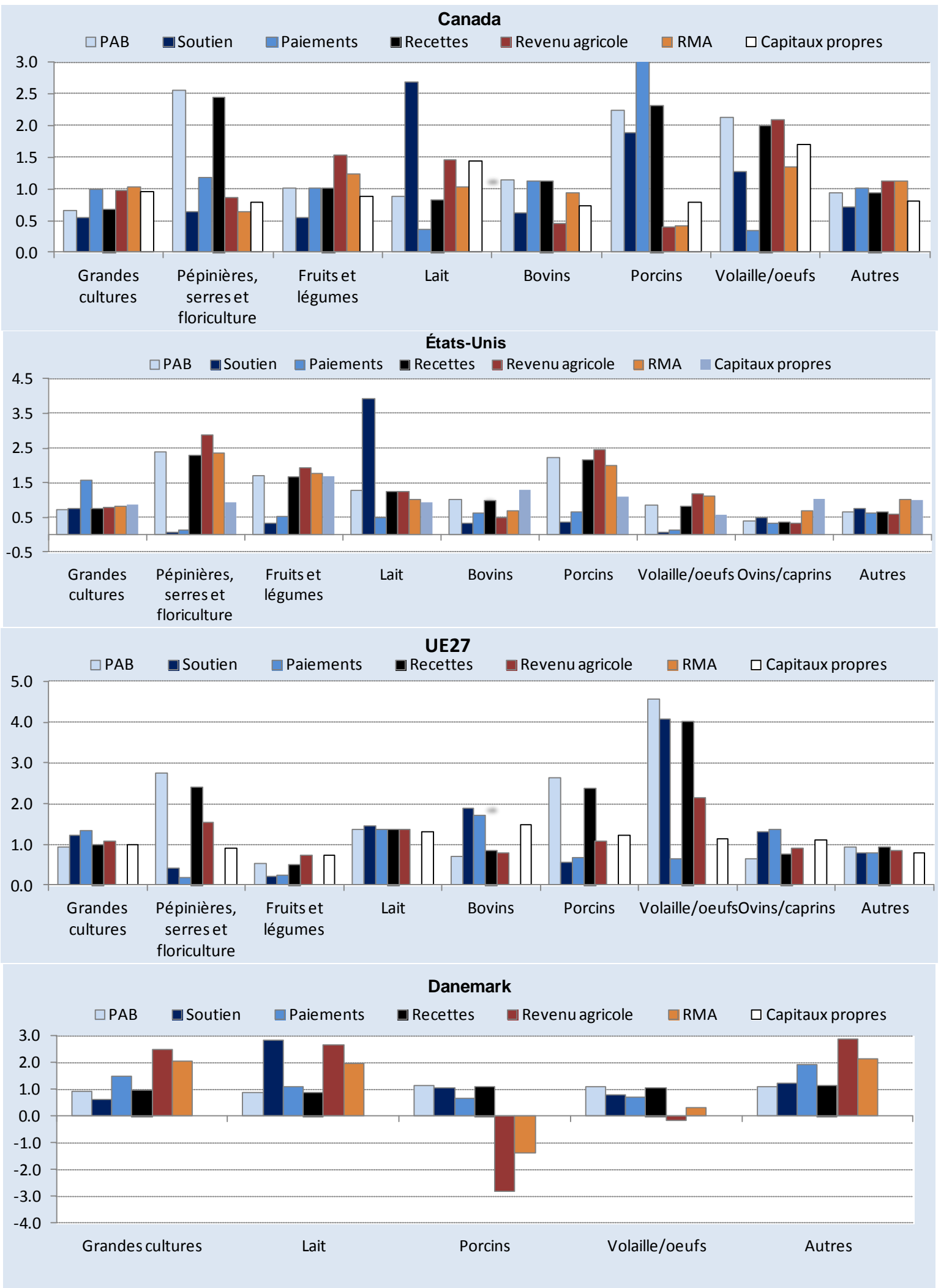




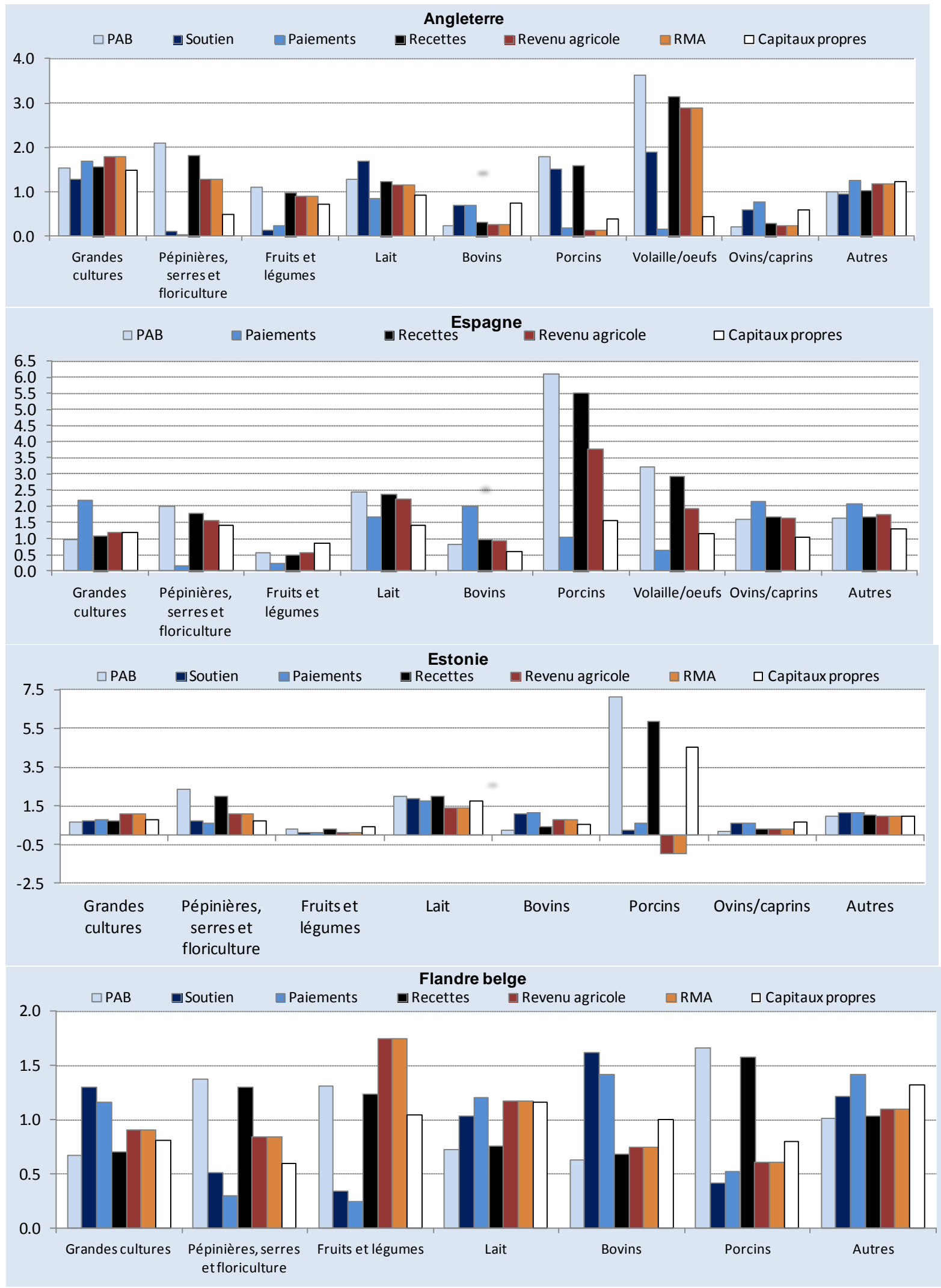




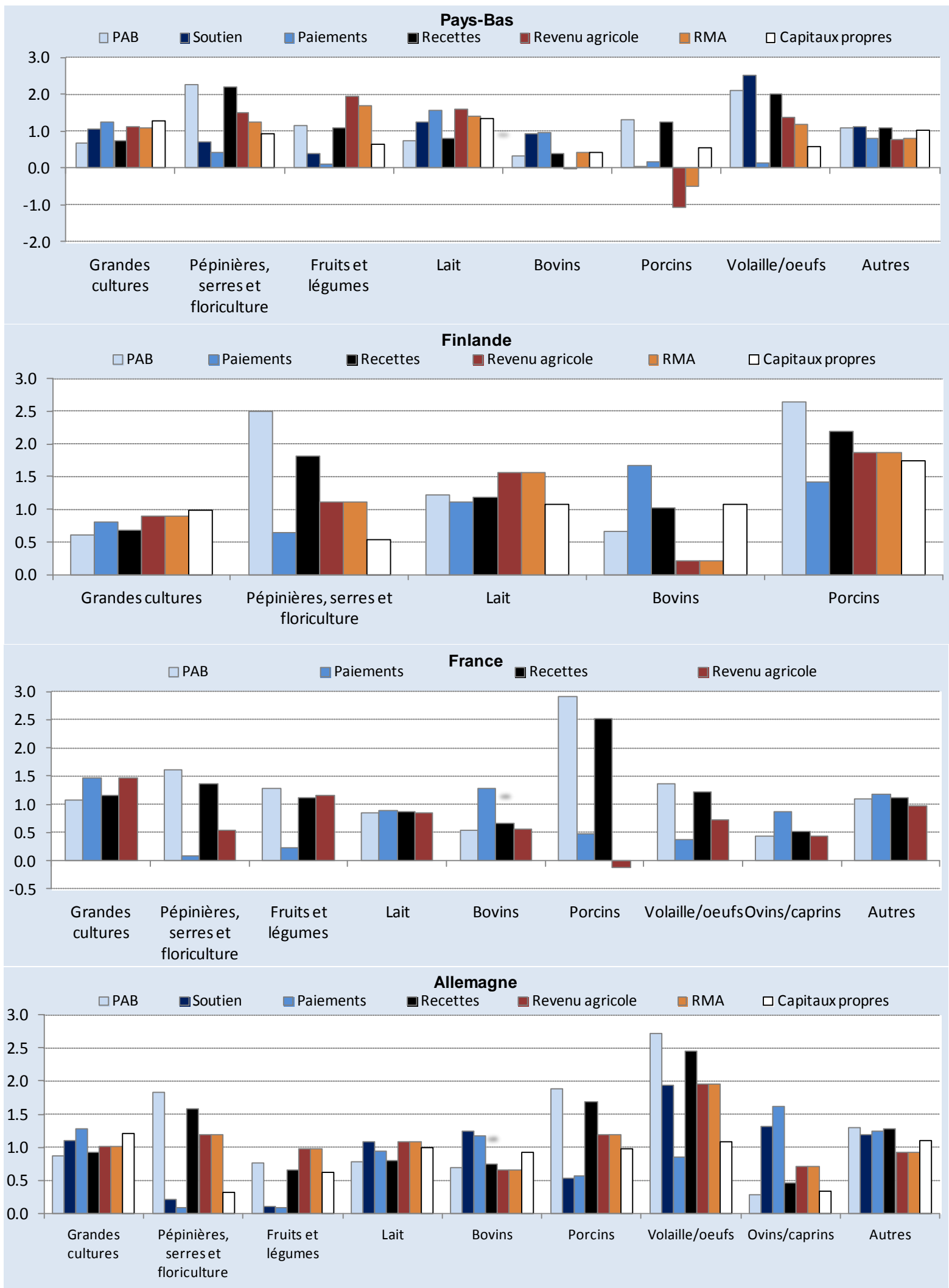




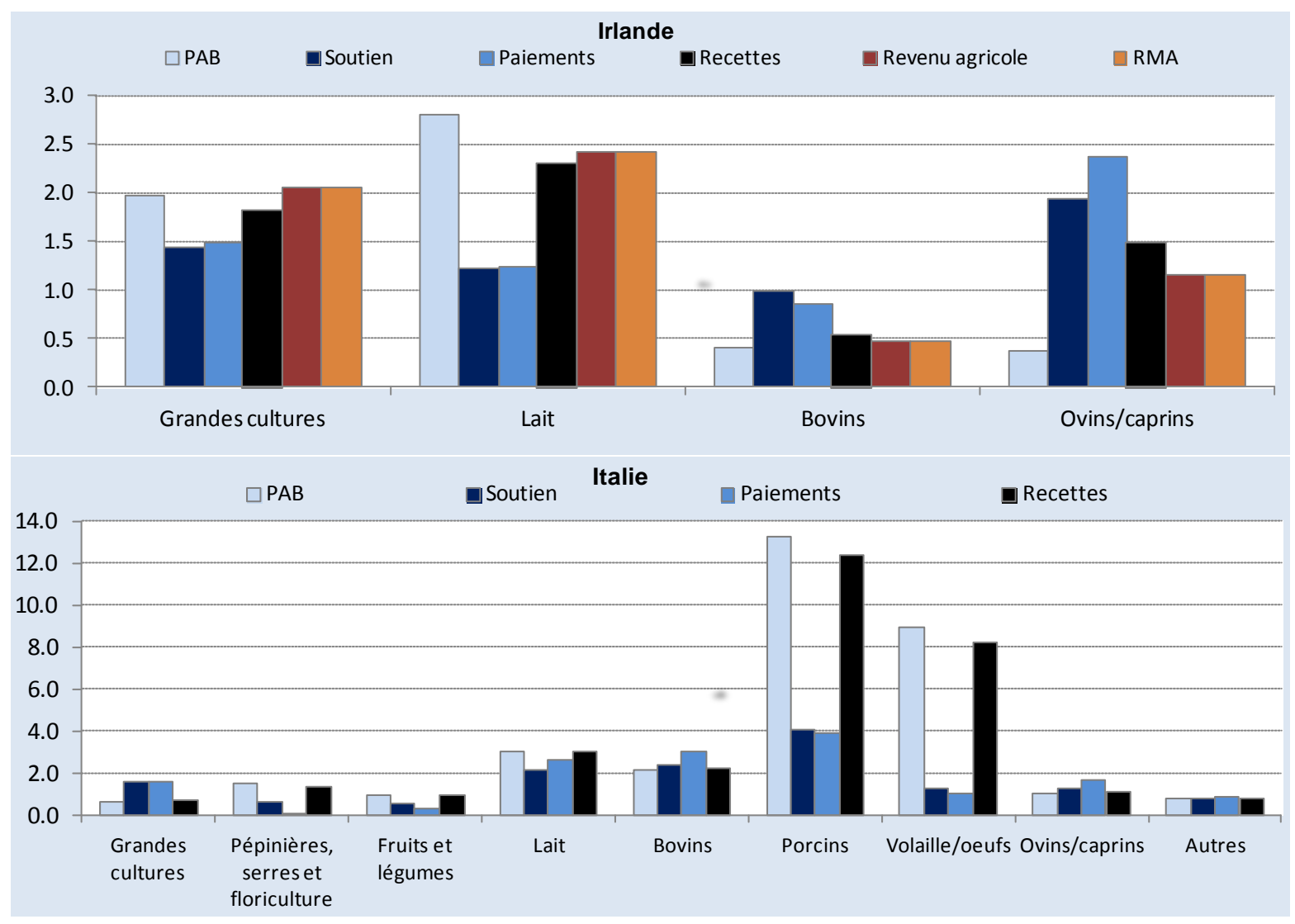

$\mathrm{PAB}$ : production agricole brute ; RMA : revenu des ménages agricoles.

Source : tableau $\mathrm{C} 5$ en annexe. 
Graphique 3.10. Part du soutien dans les recettes agricoles brutes, par type d'exploitations, 2007

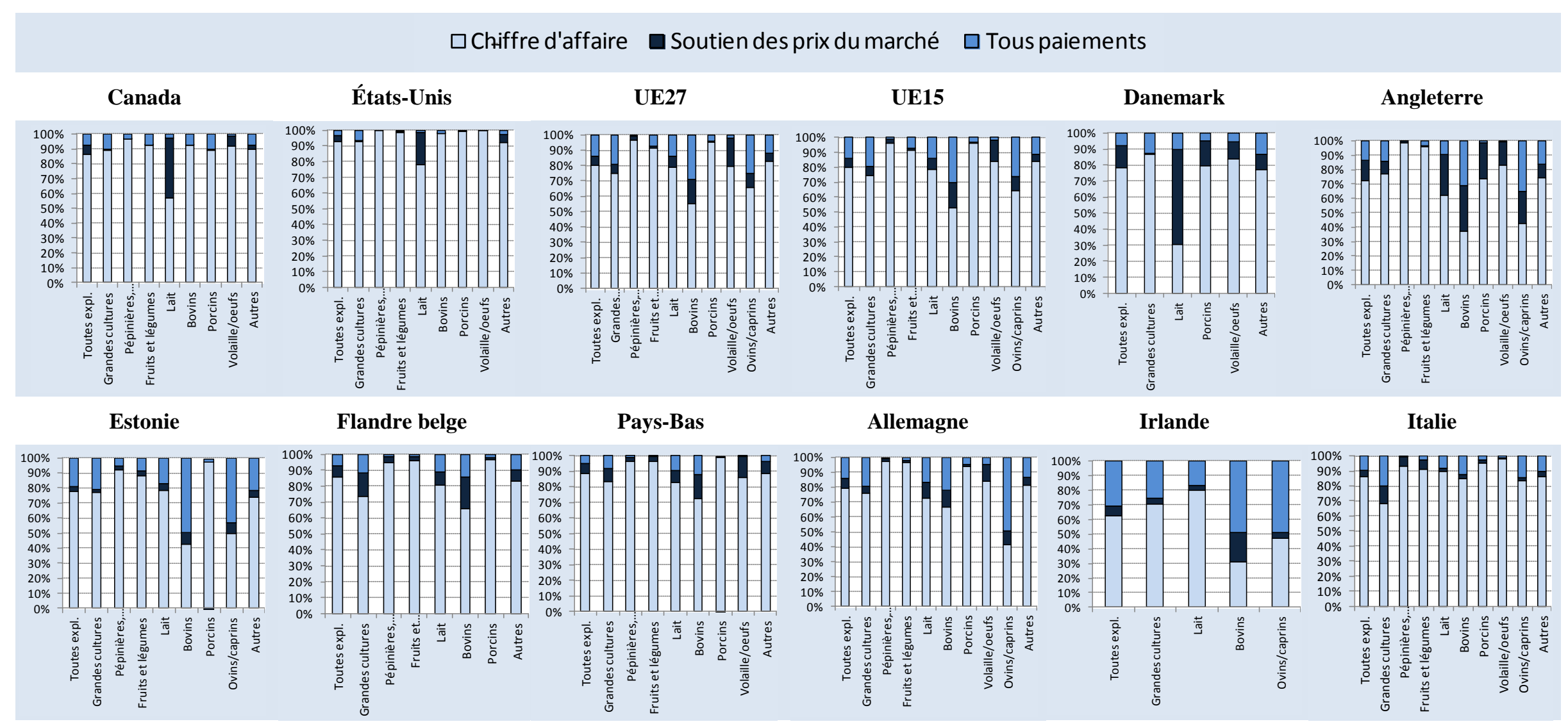

Les chiffres relatifs à l'Espagne, à la Finlande et à la France n'ont pas été inclus, car le SPM n'était pas disponible.

Source : tableau C6 en annexe. 


\section{Différences selon la région}

L'Allemagne, l'Angleterre, le Canada, les États-Unis et l'Italie ont fourni des données par type de régions conformément à leur typologie nationale ou à la typologie régionale de l'OCDE, qui est fondée sur la densité de population de la région et la présence d'un centre urbain (OCDE, 2009a). Résultat : environ $80 \%$ des exploitations sont considérées comme étant essentiellement rurales en Angleterre et au Canada, $40 \%$ aux États-Unis, $30 \%$ en Allemagne et $25 \%$ en Italie.

Au Canada, les exploitations des régions essentiellement rurales représentent environ $75 \%$ de la production agricole brute, absorbent environ $73 \%$ du soutien des prix du marché et $80 \%$ des paiements. Celles des régions essentiellement urbaines représentent $6 \%$ des exploitations et moins de $10 \%$ de la production agricole brute, et concentrent approximativement $6 \%$ du soutien total.

En Angleterre, au Canada et en Italie, la part moyenne du soutien, en particulier des paiements, dans les recettes agricoles brutes est plus faible lorsque les exploitations se trouvent dans des régions essentiellement urbaines qu'au niveau national (graphique 3.12). En Allemagne et aux États-Unis, c'est l'inverse qui se produit, probablement parce qu'elles ont une orientation technico-économique recevant davantage de paiements en Allemagne, et de SPM aux États-Unis (graphique 3.9, tableau C7 en annexe).

Au Canada, aux États-Unis et en Italie, la production agricole brute moyenne des exploitations se trouvant dans des régions essentiellement urbaines est supérieure à la moyenne nationale. A part en Allemagne, les exploitations des régions essentiellement rurales sont comparativement plus petites que celles d'autres types de régions (graphique 3.11). Aux États-Unis, les exploitations des régions considérées comme essentiellement urbaines sont de taille supérieure à celle des exploitations d'autres régions et reçoivent davantage de soutien total, mais des niveaux de paiements inférieurs à la moyenne nationale. Elles dérivent également une plus grande part de leur chiffre d'affaire du SPM, probablement du fait de l'importance des exploitations laitières (graphique 3.9). Les exploitations des régions essentiellement rurales et intermédiaires sont de taille très proche et ont un chiffre d'affaire similaire. Cependant, les exploitations des régions essentiellement rurales reçoivent davantage de paiements et moins de SPM.

En Angleterre, les niveaux moyens de soutien total et de paiements sont supérieurs à la moyenne de ceux des exploitations des régions essentiellement rurales (graphique 3.11). Ce phénomène s'explique probablement par l'importance des élevages bovins et ovins dans ces régions (graphique 3.9). En Italie, pays relativement urbanisé, les exploitations des régions intermédiaires reçoivent d'avantage de soutien que la moyenne nationale mais la part du soutien, notamment les paiements, dans les recettes agricoles brutes est plus élevée dans les régions rurales que dans d'autres types de régions, ce qui pourrait s'expliquer par les paiements au titre du second pilier, notamment en faveur des agriculteurs des zones défavorisées (graphique 3.12).

Globalement, les écarts de revenu agricole moyen entre types de régions sont inférieurs à $10 \%$. Au Canada, ce sont les régions intermédiaires qui affichent le revenu agricole moyen le plus élevé et les régions essentiellement urbaines où il est le plus bas. Bien que la production agricole brute des exploitations des régions essentiellement urbaines soit plus élevée que celle des exploitations des régions essentiellement rurales, le revenu agricole moyen y est inférieur en raison de niveaux de dépenses agricoles et d'amortissements nettement supérieurs. En Allemagne, ce sont les régions essentiellement rurales qui affichent le revenu agricole moyen le plus élevé, tandis qu'en Angleterre, ce sont les régions intermédiaires. La hiérarchie entre types de régions se 
maintient en Allemagne entre production agricole brute et revenu agricole, bien que les paiements effectués dans les régions essentiellement rurales réduisent les écarts de revenu avec les régions intermédiaires. Aux États-Unis, les exploitations des régions essentiellement urbaines ont en moyenne des revenus agricoles bien supérieurs à ceux enregistrés dans d'autres régions, et cette différence de revenu régionale persiste lorsque les revenus non agricoles sont pris en compte.

Graphique 3.11. Différences selon le type de régions, 2007

Ratio de la moyenne des exploitations des différents types de régions sur la moyenne de l'ensemble des exploitations

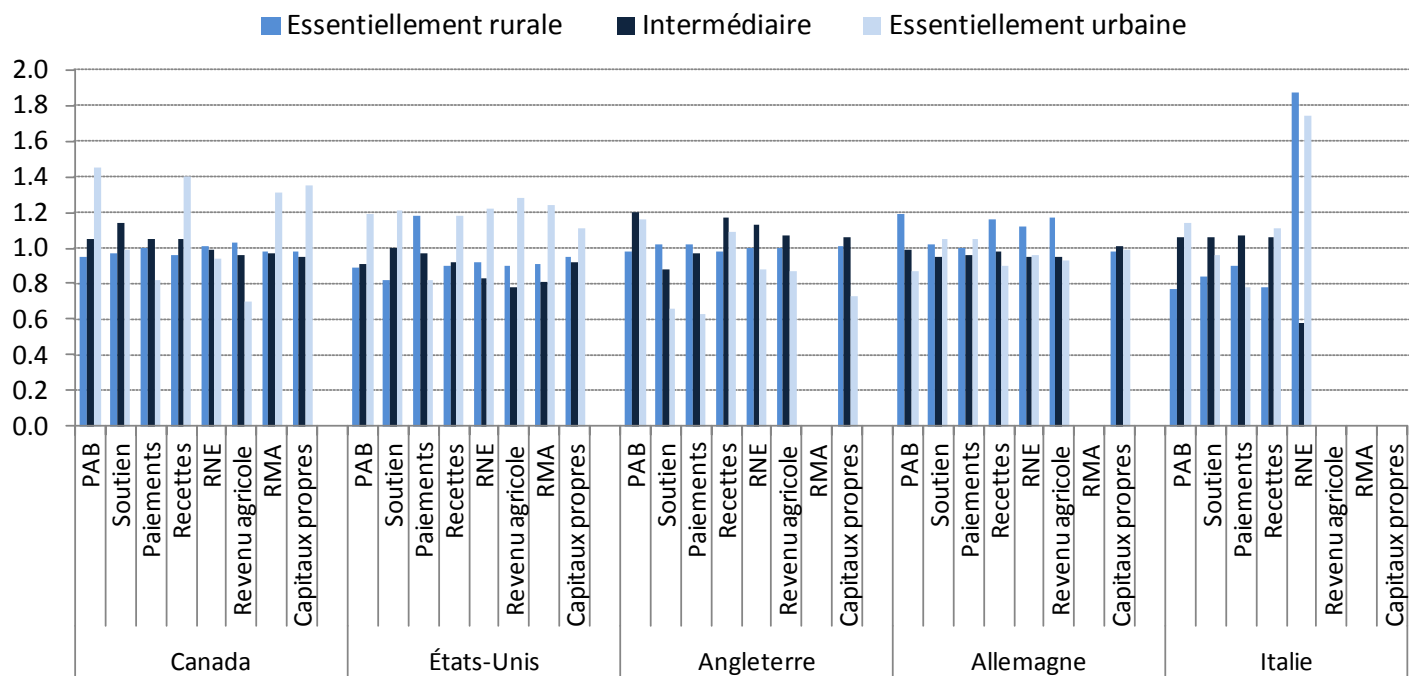

$\mathrm{PAB}$ : production agricole brute ; RNE : revenu net d'exploitation.

Source : tableau $\mathrm{C} 7$ en annexe.

Graphique 3.12. Part du soutien dans les recettes agricoles brutes, par type de régions, 2007

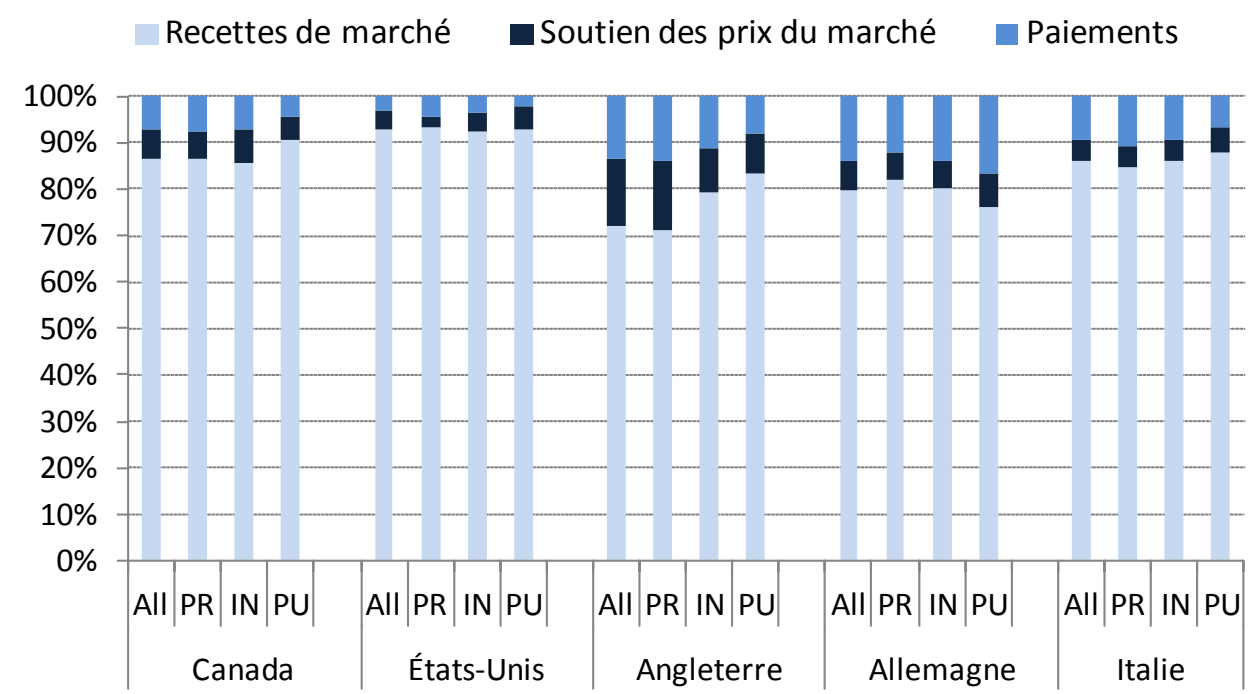

All : ensemble des exploitations couvertes ; PR : exploitations des régions essentiellement rurales ; IN : exploitations des régions intermédiaires; $\mathrm{PU}$ : exploitations des régions essentiellement urbaines.

Source : tableau C8 en annexe. 


\section{Inégalité générale}

L'inégalité est habituellement mesurée par l'indice de Gini (encadré 2). Contrairement à d'autres mesures, cet indice peut être calculé sans classement ni regroupement préalable des exploitations, et il combine au sein d'un même indicateur toutes les sources d'inégalité (taille et type d'exploitations, régions ou toute autre caractéristique). Le graphique 3.13 et le tableau C9 en annexe font apparaître que le soutien total est aussi inégal que la production agricole en Allemagne. Il l'est davantage au Canada, dans l'Union européenne, en Flandre belge, aux Pays-Bas (en 2004 et 2007) et aux États-Unis (comparé aux recettes brutes), mais il est distribué de manière plus égale que la production agricole en Angleterre et en Estonie. Cette situation révèle nécessairement des différences dans le taux de soutien appliqué aux produits, et donc aux différents types d'exploitations. Dans les États membres de l'Union européenne, l'inégalité est particulièrement forte pour les paiements du second pilier, dont le principal objectif est de remédier aux problèmes agroenvironnementaux (graphique 3.14). S'agissant des paiements du premier pilier, l'inégalité de distribution est légèrement réduite par la mise en œuvre du régime de paiement unique dans la plupart des États membres de l'Union européenne inclus dans l'analyse (tableau C9 en annexe). Elle diminue également dans la zone UE10 et est stable dans la zone UE15. Dans la zone UE27, elle décroît entre 2004 et 2006, mais augmente ensuite avec l'adhésion de la Bulgarie et de la Roumanie. En Allemagne, les différences s'estompent progressivement en raison du choix du pays de distribuer une part croissante du paiement sous forme de paiement forfaitaire par hectare (modèle hybride dynamique).

De façon générale, le soutien semble avoir un léger effet redistributif, car l'indice de Gini des recettes brutes (production agricole brute, plus total des paiements) est un peu inférieur à celui de la production agricole brute (de 0.01 dans la zone UE27, en Allemagne, aux Pays-Bas et aux États-Unis, et jusqu'à 0.04 en Angleterre) (tableau C9 en annexe).

Graphique 3.13. Comparaison des indices de Gini par pays et par composante des recettes, 2007
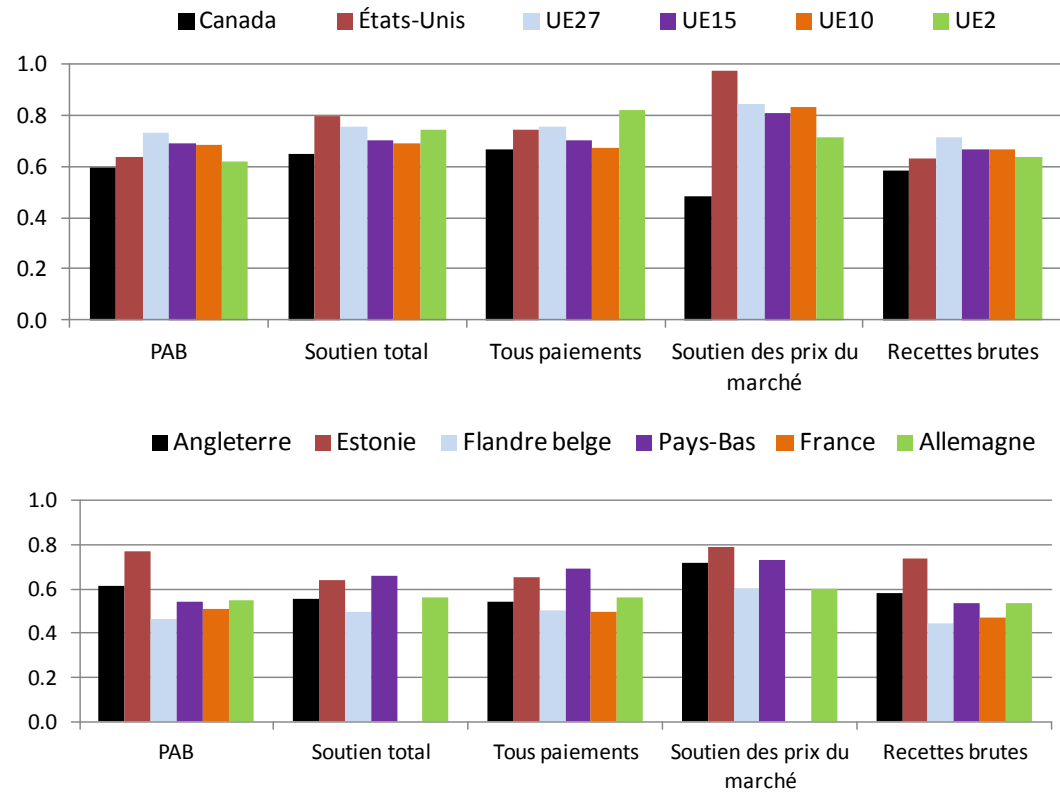

$\mathrm{PAB}$ : production agricole brute. Source : tableau C10 en annexe. 
Graphique 3.14. Comparaison des indices de Gini par pays de l'Union européenne et par type de paiements, 2007
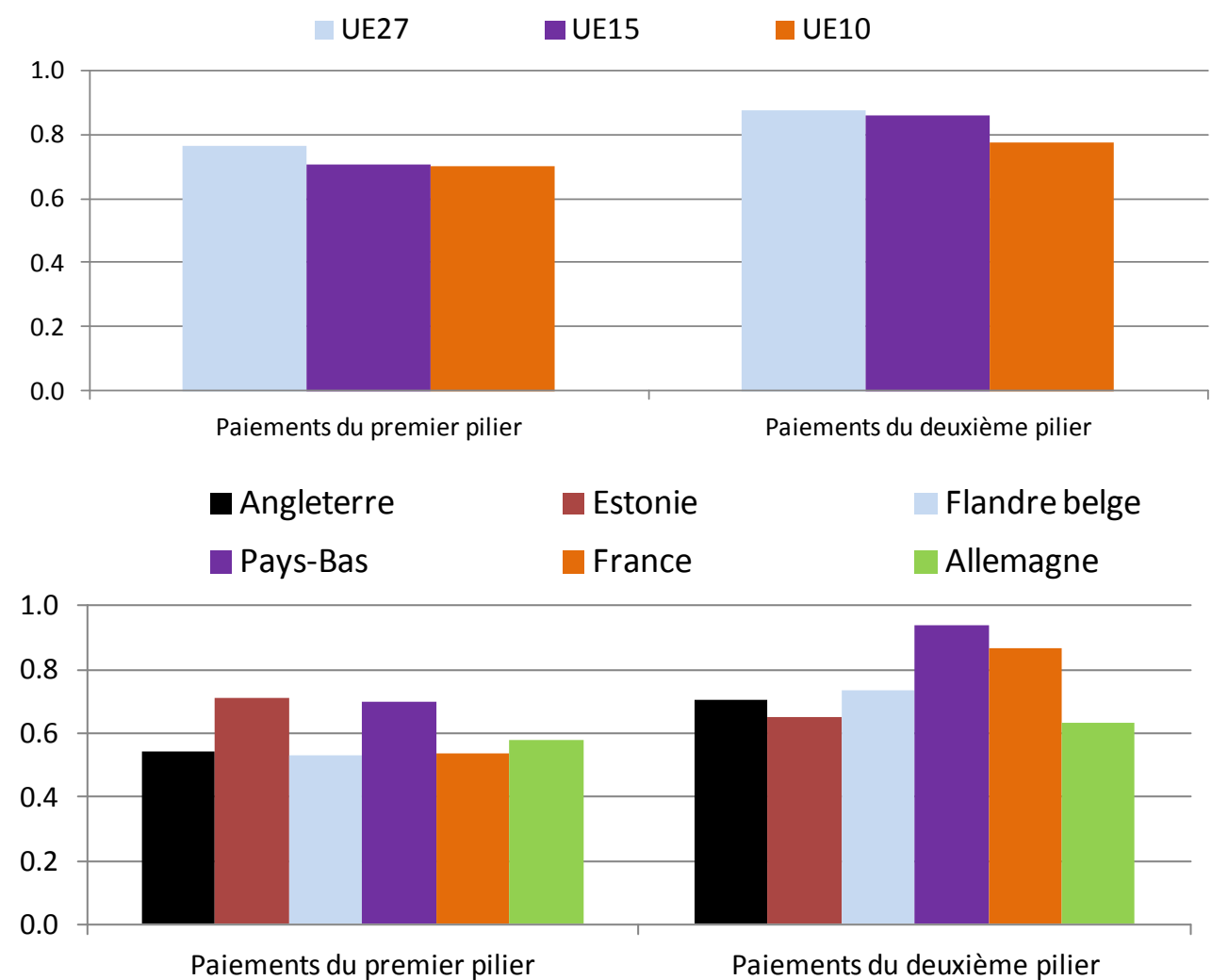

Source : tableau C9 en annexe.

\section{Résumé des principales constatations}

Il est possible de tirer certaines conclusions sur la distribution du soutien, y compris en relation avec celle des revenus, pour les quatorze pays examinés.

- On observe dans les pays sélectionnés une concentration de la production, du soutien et du revenu agricoles.

- Les $25 \%$ d'exploitations les plus grandes réalisent entre 45 et $85 \%$ de l'ensemble de la production agricole brute, représentent entre 50 et $75 \%$ du revenu agricole et reçoivent entre 35 et $75 \%$ du soutien aux exploitations agricoles.

- En dépit de sa distribution inégale, le soutien réduit légèrement l'inégalité de la distribution du revenu agricole par taille d'exploitation, car il est moins inégalement réparti que la production agricole brute et rend la distribution des recettes brutes légèrement plus égale que celle de la production agricole brute.

- Les plus petites exploitations sont plus dépendantes de la part relativement faible du soutien qu'elles reçoivent, celui-ci représentant une plus grande partie de leurs recettes agricoles brutes. 
- Les paiements totaux sont plus également distribués que le soutien des prix du marché, en partie grâce aux paiements qui ne sont pas destinés à soutenir le revenu agricole.

- Dans de nombreux pays, le soutien est concentré sur certains types d'exploitations (grandes cultures, production laitière, bovins et exploitations mixtes, principalement), ce qui reflète à la fois des différences dans le taux de soutien et l'importance de ces types d'exploitations dans la population étudiée.

- Dans la plupart des pays, le soutien réduit les écarts de revenu agricole entre les types d'exploitations car ceux qui ont des revenus plus faibles sont souvent plus soutenus.

- Toujours selon le type d'exploitations, on constate de grandes différences entre la distribution du soutien des prix du marché et celle des paiements.

- Les différences entre types d'exploitations sont également importantes en termes de revenu agricole moyen ainsi que de niveau et de composition du soutien; elles sont même plus marquées encore que selon la taille d'exploitation.

- D'une année sur l'autre, les écarts de soutien par type d'exploitations varient davantage que les autres agrégats en fonction des conditions du marché.

- Les différences de revenu agricole entre les types de régions ne sont pas importantes, et le soutien semble les atténuer.

- Lorsqu'il est pris en compte, le revenu non agricole réduit les écarts de revenus entre les ménages agricoles.

- La distribution des dettes agricoles est proche de celle du revenu agricole ou légèrement plus inégale, mais celle des capitaux propres n'est pas aussi inégale, car les exploitations plus petites sont proportionnellement moins endettées que les plus grandes.

- Indépendamment des groupes d'exploitations, le soutien total a un léger effet redistributif sur le revenu agricole car l'indice de Gini du chiffre des recettes brutes, paiements compris, est marginalement inférieur à celui de la production agricole brute.

Ces constatations rejoignent les conclusions de travaux précédents. Tandis que les rapports de l'OCDE précédents (OCDE, 1999, 2003) s'intéressait à la distribution du soutien et des revenus selon la taille des exploitations, la présente analyse examine plus systématiquement les différences de ces distributions selon le type d'exploitations et de régions. Bien qu'elle ne soit pas parfaite, l'harmonisation des définitions de la population totale et des groupes d'exploitations facilite également les comparaisons entre pays.

\section{Implications en termes de politiques}

Que ce soit pour des questions d'efficience ou d'équité, il est important, lorsqu'on évalue des politiques, de déterminer si le soutien bénéficie aux exploitations auxquelles il était destiné, par exemple aux agriculteurs qui ont de faibles revenus dans le cas du soutien du revenu, ou à ceux qui fournissent le niveau requis d'avantages environnementaux ou sociaux. Une politique qui offre un appui notable à des bénéficiaires non visés n'est ni efficiente (gaspillage d'argent) ni équitable (l'argent pourrait être mieux utilisé ailleurs). Pour détecter ces bénéficiaires non visés, il faut avoir 
correctement défini les objectifs, avec des cibles claires et mesurables, et disposer des informations nécessaires pour évaluer la situation, déterminer les cibles et mesurer les effets de la politique.

La distribution du soutien doit être considérée en relation avec les objectifs des politiques. Il est donc utile de savoir comment se distribuent soutien et revenus, afin de vérifier dans quelle mesure l'aide au revenu bénéficie aux agriculteurs qui en ont besoin, et à quel point les écarts de soutien influent sur la compétitivité des exploitations et le bien-être des agriculteurs. Toutefois, les informations sur le niveau de revenu des agriculteurs sont souvent incomplètes et difficiles d'accès, ce qui ne permet pas de diagnostiquer facilement la cause et l'étendue des problèmes de revenu dans l'agriculture, et de les comparer à la situation dans d'autres secteurs. Pour qu'il augmente l'équité, le soutien du revenu devrait profiter aux bas revenus et donc rendre la distribution du revenu plus égale. Mais les autres mesures, qui ne sont pas associées à des objectifs redistributifs explicites ou implicites, ne doivent pas forcément être réparties également : elles sont équitables dans la mesure où elles sont ciblées et où le soutien est lié à la valeur des biens et services fournis. Tout en étant équitables, elles peuvent être inégalement distribuées si les exploitations rendent des services d'importance différente. Comme toujours en matière d'argent public, il peut y avoir des limites au degré d'inégalité que la société est prête à accepter pour la rémunération de services et les autorités doivent en tenir compte. En outre, quel que soit l'objectif, les autorités doivent s'assurer que les distorsions imposées au marché sont minimales, c'est-à-dire que les paiements sont aussi découplés que possible.

Cette étude montre que le soutien contribue à augmenter le revenu agricole et à améliorer légèrement sa distribution, avec cependant le gros du soputien allant aux plus grandes exploitations, qui enregistrent des revenus agricoles supérieurs à la moyenne. Dans la mesure où une grande part du soutien est souvent associée à des objectifs de revenu, cela représente d'importantes fuites vers des bénéficiaires non visés comme les agriculteurs dont les revenus sont supérieurs à la moyenne. La forte corrélation entre soutien et taille économique des exploitations indique un manque de ciblage de la plupart des mesures de soutien. Pour qu'une politique de soutien du revenu soit efficiente et équitable, elle doit cibler un indicateur du revenu, de préférence au niveau individuel, de sorte que seuls les bénéficiaires prévus reçoivent un soutien et que ce dernier soit adapté à leur situation particulière. Toute autre cible entraînera des fuites et des inefficiences importantes, à moins qu'elle ne soit fortement corrélée à la question du revenu. Afin d'élaborer des politiques plus efficientes et plus équitables, les gouvernements ont besoin d'informations de meilleure qualité sur les revenus et la situation patrimoniale des ménages agricoles. 


\section{Références}

OCDE (1998), L'Agriculture dans un monde en mutation: quelles politiques pour demain? Communiqué de presse, Réunion du Comité de l'agriculture au niveau ministériel [SG/COM/NEWS(98)22], 5-6 mars, Paris.

OCDE (1999), Les effets redistributifs du soutien agricole dans quelques pays de l'OCDE, OCDE, Paris.

OCDE (2003), Le revenu des ménages agricoles. Problèmes et réponses, [AGR/CA/APM(2002)11/FINAL], OCDE, Paris, www.oecd.org/document/20/0,3343,fr_2649_33773_20702868_1_1_1_1,00.html.

OCDE (2005a), Les politiques agricoles des pays de l'OCDE : Suivi et évaluation 2009, OCDE, Paris.

OCDE (2009a), Le rôle de l'agriculture et de la diversification des ménages agricoles dans l'économie des régions rurales: État des lieux et conséquences initiales pour l'action publique, [TAD/CA/APM/WP(2009)1/FINAL], OCDE, Paris, www.oecd.org/dataoecd/28/44/42714347.pdf.

OCDE (2011), «Évaluation des réformes de la politique agricole de l'Union européenne », OCDE, Paris. 


\section{Annexe A.}

\section{Liste des tableaux fournis par les participants}

\section{Il a été demandé aux participants de fournir les tableaux suivants :}

Revenu total dans certains pays de l'OCDE, par composante du revenu et quartile, 2004, 2006 et 2007

Revenu total de l'ensemble des exploitations dans certains pays de l'OCDE, par composante du revenu et type d'exploitations, 2004, 2006 et 2007

Revenu total des exploitations du premier quartile dans certains pays de l'OCDE, par composante du revenu et type d'exploitations, 2004, 2006 et 2007

Revenu total des exploitations du quatrième quartile dans certains pays de l'OCDE, par composante du revenu et type d'exploitations, 2004, 2006 et 2007

Revenu total de l'ensemble des exploitations dans certains pays de l'OCDE, par composante du revenu et région, 2004, 2006 et 2007

Revenu total dans certains pays de l'OCDE, par composante du revenu et par quartile défini sur la base du soutien, 2004, 2006 et 2007

Indices de Gini dans certains pays de l'OCDE, par composante du revenu, 2004, 2006 et 2007 


\section{Annexe B.}

\section{Sources de données}

\section{Canada - Fichier du Programme des données fiscales (PDF), pour les indicateurs relatifs aux exploitations et les indicateurs familiaux}

Organisation : Statistique Canada (StatCan), Division de l'agriculture.

Description: le Programme des données fiscales échantillonne les déclarations de revenus des exploitations agricoles constituées en société et des exploitations non constituées en société pour produire des estimations pour un ensemble de variables financières (détail des revenus et des dépenses et du revenu non agricole des exploitants).

Champ d'application : la population cible comprend toutes les exploitations agricoles constituées ou non en société et des organisations communautaires agricoles au Canada. La base de sondage des exploitations agricoles non constituées en société inclut tous les particuliers qui déclarent soit un revenu agricole brut positif, soit un revenu agricole net non nul au titre d'un travail indépendant sur leur formulaire T1 Générale de l'ARC Déclaration de revenus et de prestations. La base de sondage des exploitations agricoles constituées en société comprend l'ensemble des sociétés à l'intérieur des dix provinces et des territoires qui déclarent des ventes brutes de $25000 \mathrm{CAD}$ et plus et qui sont classées comme exploitation agricole $(50 \%$ ou plus des ventes doivent provenir d'activités agricoles) conformément au Système de classification des industries de l'Amérique du Nord (SCIAN). La base comprend également l'ensemble des organisations communautaires agricoles qui déclarent soit un revenu agricole brut positif, soit un revenu agricole net non nul sur leur formulaire T3 de l'ARC Déclaration de renseignements et de revenus des fiducies.

Elle n'inclut pas les déclarants dans plusieurs juridictions (plus d'une province), les résidents non canadiens ou les sociétés non résidentes.

Taille de l'échantillon : environ 180000 exploitations non constituées en société, 20000 exploitations constituées en société et 300 organisations communautaires agricoles.

Champ d'application du projet du Réseau de l'OCDE sur l'analyse au niveau de l'exploitation : les exploitations retenues pour le projet représentent $90 \%$ de la production agricole brute nationale et enregistrent plus de $100000 \mathrm{CAD}$ de chiffre d'affaires brut.

Domaines : $\quad$ a-Types d'exploitations définis par le Système de classification des industries de l'Amérique du Nord (SCIAN)

b- Taille fondée sur le revenu agricole brut c-Statut juridique : exploitations non constituées en société, exploitations constituées en société et organisations communautaires agricoles

d- Géographie : le niveau le plus détaillé est la Région agricole de recensement 
Publications : $\quad$ Statistique Canada, Statistiques sur les revenus des exploitants agricoles - 21-206-X Statistique Canada, Statistiques sur les revenus des familles agricoles - 21-207-X Statistique Canada, Statistiques sur les revenus et les dépenses des exploitations agricoles - 21-208-X

Accessibilité : les données sont collectées par l'Agence du revenu du Canada et ne peuvent pas être communiquées, conformément au mémorandum d'accord entre Statistique Canada et l'Agence du revenu du Canada. Elles sont accessibles aux employés de Statistique Canada et aux personnes considérées comme employées, sous contrat de recherche.

Questionnaire : sans objet

Métadonnées : veuillez vous référer aux informations du site Web de Statistique Canada relatives aux publications mentionnées ci-dessus. Statistique Canada, Base de données complètes sur les exploitations agricoles : manuel de référence - 21F0005G

\section{Canada - Enquête financière sur les fermes, pour les indicateurs d'actifs, de dettes et de capitaux propres agricoles}

Organisation : Statistique Canada, Division de l'agriculture.

Description : cette enquête recueille des données sur les exploitations agricoles, en particulier sur l'utilisation des terres, les achats et les ventes de biens immobilisés, les actifs, les dettes, les emprunts, les recettes et les dépenses. Des questions concernent le revenu tiré de sources autres que l'exploitation agricole.

Champ d'application : la population cible de cette enquête comprend toutes les fermes canadiennes qui étaient exploitées à la fin de l'année de référence. Elle se compose d'exploitations, et non de ménages ou de familles.

Certaines exploitations ont été exclues de la population cible, notamment celles dont le chiffre des ventes de produits agricoles était inférieur à $10000 \mathrm{CAD}$, les fermes institutionnelles, les pâturages communautaires, les fermes situées sur des réserves indiennes et celles appartenant à des entreprises à exploitations agricoles multiples.

Taille de l'échantillon : 14000 exploitations agricoles environ, selon l'année.

Domaines: a-Types d'exploitations définis par le Système de classification des industries de l'Amérique du Nord (SCIAN)

b- Famille agricole ${ }^{15}$

c- Taille fondée sur le revenu agricole brut

d- Géographie : le niveau le plus détaillé est la Région agricole de recensement e- Statut juridique :

secteur des exploitations agricoles non constituées en société : entreprise à propriétaire unique, société en nom collectif, coopérative et exploitations communautaires ; secteur des exploitations agricoles constituées en société : corporation.

Publication : $\quad$ Statistique Canada, Enquête financière sur les fermes (21F0008X)

15. La famille comprend l'exploitant, son conjoint et les enfants non mariés résidant dans le même foyer. 
Accessibilité : les données sont collectées en vertu de la Loi sur la statistique. Elles sont accessibles aux employés de Statistique Canada et aux personnes considérées comme employées, sous contrat de recherche.

Un fichier de microdonnées (sans identifiants) des sondés qui acceptent de partager les informations qu'ils ont communiquées lors de l'enquête est fourni à Agriculture et Agroalimentaire Canada à des fins de recherche et de statistiques.

Questionnaire et métadonnées : veuillez vous référer aux informations du site Web de Statistique Canada relatives à la publication mentionnée ci-dessus.

Contact : $\quad$ Martin S. Beaulieu, Chef de la Section des projets des données complètes sur les exploitations agricoles

Adresse électronique : martin.beaulieu@ statcan.ca 


\section{États membres de l'Union européenne - Réseau d'information comptable agricole (RICA)}

Le réseau européen RICA est présenté sur le site ec.europa.eu/agriculture/rica/index_fr.cfm.

Des informations sur la méthodologie sont fournies sur la page ec.europa.eu/agriculture/rica/methodology2_fr.cfm.

Le RICA exclut les exploitations qui n'atteignent pas une dimension économique minimale. Cette taille est exprimée en unités de dimension européenne (UDE), qui permettent de mesurer la marge brute (standard). Les seuils appliqués dans les États membres de l'Union européenne sont indiqués ci-dessous.

\begin{tabular}{|c|c|}
\hline \multicolumn{2}{|c|}{$\begin{array}{c}\text { Seuils de dimension économique appliqués par la Commission (en UDE) } \\
\text { à partir de l'année } 2007\end{array}$} \\
\hline Belgique & 16 \\
\hline Bulgarie & 1 \\
\hline République tchèque & 4 \\
\hline Danemark & 8 \\
\hline Allemagne & 16 \\
\hline Estonie & 2 \\
\hline Irlande & 2 \\
\hline Grèce & 2 \\
\hline Espagne & 2 \\
\hline France & 8 \\
\hline Italie & 4 \\
\hline Chypre & 2 \\
\hline Lettonie & 2 \\
\hline Lituanie & 2 \\
\hline Luxembourg & 8 \\
\hline Hongrie & 2 \\
\hline Malte & 8 \\
\hline Pays-Bas & 16 \\
\hline Autriche & 8 \\
\hline Pologne & 2 \\
\hline Portugal & 2 \\
\hline Roumanie & 1 \\
\hline Slovénie & 2 \\
\hline Slovaquie & 8 \\
\hline Finlande & 8 \\
\hline Suède & 8 \\
\hline Royaume-Uni & 16 \\
\hline Royaume-Uni (Irlande du Nord) & 8 \\
\hline
\end{tabular}


Selon les États membres, la couverture du RICA diffère de celle de l'Enquête sur la structure des exploitations agricoles (ESA) publiée par Eurostat, comme le montre le tableau ci-après.

\begin{tabular}{|c|c|c|c|c|c|c|c|}
\hline \multicolumn{8}{|c|}{ Année de l'Enquête sur la structure des exploitations agricoles (ESA) 2007} \\
\hline \multirow{2}{*}{ État membre } & \multicolumn{2}{|c|}{ Exploitations ESA } & \multicolumn{5}{|c|}{ Champ d'observation RICA } \\
\hline & Total & Champ RICA & Exploitations (\%) & UDE (\%) & SAU (\%) & UTA (\%) & MBS \\
\hline Belgique & 48010 & 32640 & 67.0 & 100.0 & 103.0 & 95.0 & 2004 \\
\hline Bulgarie & 493130 & 117790 & 24.0 & 89.0 & 98.0 & 58.0 & 2004 \\
\hline République tchèque & 39390 & 14670 & 37.0 & 103.0 & 99.0 & 88.0 & 2004 \\
\hline Danemark & 44630 & 32790 & 72.0 & 103.0 & 99.0 & 90.0 & 2004 \\
\hline Allemagne & 370470 & 187130 & 50.0 & 93.0 & 92.0 & 70.0 & 2004 \\
\hline Estonie & 23340 & 7310 & 31.0 & 87.0 & 101.0 & 62.0 & 2004 \\
\hline Irlande & 128260 & 107460 & 83.0 & 95.0 & 116.0 & 82.0 & 2004 \\
\hline Grèce & 860150 & 568010 & 63.0 & 94.0 & 93.0 & 111.0 & 2004 \\
\hline Espagne & 1043920 & 823580 & 70.0 & 88.0 & 84.0 & 103.0 & 2004 \\
\hline France & 527350 & 375130 & 67.0 & 97.0 & 100.0 & 85.0 & 2004 \\
\hline Italie & 1679430 & 760650 & 44.0 & 98.0 & 99.0 & 81.0 & 2004 \\
\hline Chypre & 40120 & 20120 & 49.0 & 87.0 & 97.0 & 90.0 & 2004 \\
\hline Lettonie & 107740 & 22810 & 21.0 & 90.0 & 80.0 & 50.0 & 2004 \\
\hline Lituanie & 230280 & 39650 & 17.0 & 73.0 & 77.0 & 44.0 & 2004 \\
\hline Luxembourg & 2310 & 1780 & 70.0 & 94.0 & 99.0 & 71.0 & 2004 \\
\hline Hongrie & 626330 & 87860 & 13.0 & 91.0 & 103.0 & 37.0 & 2004 \\
\hline Malte $\left(^{*}\right)$ & 11010 & 1480 & 13.0 & 70.0 & 47.0 & 66.0 & 2004 \\
\hline Pays-Bas $\left(^{*}\right)$ & 76740 & 59240 & 77.0 & 106.0 & 101.0 & 99.0 & 2004 \\
\hline Autriche & 165430 & 74910 & 44.0 & 87.0 & 76.0 & 71.0 & 2004 \\
\hline Pologne & 2390970 & 766730 & 32.0 & 88.0 & 85.0 & 59.0 & 2004 \\
\hline Portugal & 275090 & 117050 & 39.0 & 77.0 & 82.0 & 52.0 & 2004 \\
\hline Roumanie & 3931350 & 866680 & 21.0 & 65.0 & 62.0 & 78.0 & 2004 \\
\hline Slovénie & 75310 & 42940 & 54.0 & 80.0 & 97.0 & 85.0 & 2004 \\
\hline Slovaquie & 69000 & 3430 & 5.0 & 92.0 & 104.0 & 64.0 & 2004 \\
\hline Finlande & 68230 & 41060 & 59.0 & 97.0 & 92.0 & 82.0 & 2004 \\
\hline Suède & 72620 & 27130 & 36.0 & 77.0 & 83.0 & 60.0 & 2004 \\
\hline Royaume-Uni & 299840 & 86210 & 29.0 & 94.0 & 87.0 & 61.0 & 2004 \\
\hline
\end{tabular}

ESA : Enquête sur la structure des exploitations agricoles ; RICA : Réseau d'information comptable agricole ; UDE : unité de dimension européenne ; SAU : superficie agricole utilisée ; UTA : unité de travail annuel ; MBS : marge brute standard.

Chaque pays membre conçoit sa propre enquête RICA, recueille ses données, puis transmet des informations standard à la Commission européenne. Par conséquent, une enquête RICA nationale peut contenir davantage d'informations que l'enquête RICA européenne. Les exploitations agricoles retenues pour le projet du Réseau de l'OCDE sur l'analyse au niveau de l'exploitation représentent $90 \%$ de la production agricole brute nationale. 


\section{Danemark}

\begin{tabular}{lcccc}
\hline \multicolumn{1}{c}{ Année } & Unité & $\mathbf{2 0 0 4}$ & $\mathbf{2 0 0 6}$ & $\mathbf{2 0 0 7}$ \\
\hline Exploitations couvertes & Nombre & 10393 & 8605 & 8448 \\
$\begin{array}{l}\text { Unités de travail annuel (équivalent } \\
\text { temps plein) }\end{array}$ & UTA & n.d. & n.d. & n.d. \\
Superficie agricole utilisée & 1000 hectares & n.d. & n.d. & n.d. \\
\hline
\end{tabular}

Angleterre

\begin{tabular}{lcrrr}
\hline \multicolumn{1}{c}{ Année } & Unité & 2004 & \multicolumn{1}{c}{$\mathbf{2 0 0 6}$} & $\mathbf{2 0 0 7}$ \\
\hline Exploitations couvertes & Nombre & 60825 & 59534 & 57089 \\
Unités de travail annuel (équivalent & UTA & 164923 & 165074 & 158172 \\
temps plein) & 1000 hectares & 8095 & 8078 & 7964 \\
Superficie agricole utilisée & 105 \\
\hline
\end{tabular}

Les données concernent uniquement l'Angleterre. La source de données est la Farm Business Survey (Enquête sur les entreprises agricoles) en Angleterre. Les exploitations de cette enquête comptent pour plus de la moitié du nombre total d'exploitations anglaises. Elles représentent $91 \%$ de la superficie exploitée et $96 \%$ de la production agricole de la nation.

\section{Estonie}

\begin{tabular}{lcrrr}
\hline \multicolumn{1}{c}{ Année } & Unité & 2004 & \multicolumn{1}{c}{$\mathbf{2 0 0 6}$} & $\mathbf{2 0 0 7}$ \\
\hline Exploitations couvertes & Nombre & 6809 & 6724 & 7301 \\
$\begin{array}{l}\text { Unités de travail annuel (équivalent } \\
\text { temps plein) }\end{array}$ & UTA & 22838 & 20481 & 20405 \\
Superficie agricole utilisée & 1000 hectares & 811 & 795 & 905 \\
\hline
\end{tabular}

En Estonie, 7301 exploitations agricoles dépassent le seuil de dimension économique (2 UDE) défini pour le RICA. Les exploitations agricoles couvertes par l'enquête RICA représentent $92.6 \%$ de la marge brute standard de la production agricole estonienne, $31.3 \%$ du nombre total d'exploitations agricoles et $87.0 \%$ de la superficie agricole utilisée. La taille de l'échantillon est de 500 exploitations. Toutes les données individuelles sont pondérées selon le type d'exploitations et la classe de dimension économique.

\section{Finlande}

\begin{tabular}{|c|c|c|c|c|}
\hline Année & Unité & 2004 & 2006 & 2007 \\
\hline Exploitations couvertes & Nombre & 45884 & 42843 & 40794 \\
\hline $\begin{array}{l}\text { Unités de travail annuel (équivalent } \\
\text { temps plein) }\end{array}$ & UTA & 69278 & 58669 & 56037 \\
\hline Superficie agricole utilisée & 1000 hectares & 2045 & 2068 & 2028 \\
\hline \multicolumn{5}{|l|}{ Flandre (Belgique) } \\
\hline Année & Unité & 2004 & 2006 & 2007 \\
\hline Exploitations couvertes & Nombre & 22574 & 21675 & 20690 \\
\hline $\begin{array}{l}\text { Unités de travail annuel (équivalent } \\
\text { temps plein) }\end{array}$ & UTA & 45374 & 44217 & 43242 \\
\hline Superficie agricole utilisée & 1000 hectares & 650 & 651 & 661 \\
\hline
\end{tabular}


Toutes les données d'exploitation individuelles sont pondérées selon le type et la taille de l'exploitation (unités de dimension économique).

\section{France}

\begin{tabular}{lcccc}
\hline \multicolumn{1}{c}{ Année } & Unité & $\mathbf{2 0 0 4}$ & $\mathbf{2 0 0 6}$ & $\mathbf{2 0 0 7}$ \\
\hline Exploitations couvertes & Nombre & 346219 & 346219 & 326008 \\
$\begin{array}{l}\text { Unités de travail annuel (équivalent } \\
\text { temps plein) }\end{array}$ & UTA & 690237 & 684802 & 651799 \\
Superficie agricole utilisée & 1000 hectares & 25836 & 26477 & 26055 \\
\hline
\end{tabular}

L'enquête RICA française est réalisée à partir d'un échantillon d'environ 7300 exploitations (7 332 pour l'année 2004, 7346 pour 2006 et 7377 pour 2007).

Revenu agricole - Résultat courant avant impôt: solde intermédiaire de gestion standardisé : production de l'exercice + subventions d'exploitation-charges d'exploitation (y compris amortissements)-charges financières nettes. Les «autres charges courantes » comprennent les postes : loyers et fermages, impôts et taxes, charges de personnel, dotations aux amortissements, charges financières. Revenu non agricole ces informations ne sont pas incluses dans l'enquête RICA. Amortissements - Dotations aux amortissements : constatation comptable d'un amoindrissement irréversible de la valeur d'un élément d'actif immobilisé, résultant de l'usage du temps, d'un changement technique ou de toute autre cause. Calcul linéaire des amortissements. Revenu net d'exploitation - Résultat courant avant impôt - Dotations aux amortissements. Le soutien des prix du marché n'est pas calculé pour la France. Production agricole brute Différence entre le Produit brut total et l'ensemble des paiements. Recettes brutes - Le Produit brut total correspond à la somme de la production de l'exercice (nette des achats d'animaux), des subventions d'exploitation, des indemnités d'assurance, des remboursements forfaitaires de TVA, des rabais, remises et ristournes obtenus, des autres produits de gestion courante et des transferts de charge. Les dépenses d'exploitation correspondent à la différence entre les recettes brutes et le revenu net d'exploitation.

\section{Allemagne}

\begin{tabular}{lcccc}
\hline \multicolumn{1}{c}{ Année } & Unité & $\mathbf{2 0 0 4}$ & $\mathbf{2 0 0 6}$ & $\mathbf{2 0 0 7}$ \\
\hline Exploitations couvertes & Nombre & 277119 & 259508 & 260882 \\
$\begin{array}{l}\text { Unités de travail annuel (équivalent } \\
\text { temps plein) }\end{array}$ & UTA & 506560 & 491412 & 501241 \\
Superficie agricole utilisée & 1000 hectares & 17654 & 16963 & 17644 \\
\hline
\end{tabular}

Les échantillons représentent environ 11500 exploitations chaque année. Toutes les données des exploitations individuelles sont pondérées en fonction des coefficients de pondération de l'année de référence.

Irlande

\begin{tabular}{lcrrr}
\hline \multicolumn{1}{c}{ Année } & Unité & $\mathbf{2 0 0 4}$ & $\mathbf{2 0 0 6}$ & $\mathbf{2 0 0 7}$ \\
\hline Exploitations couvertes & Nombre & 113261 & 113068 & 111913 \\
$\begin{array}{l}\text { Unités de travail annuel (équivalent } \\
\text { temps plein) }\end{array}$ & UTA & n.d. & n.d. & n.d. \\
Superficie agricole utilisée & 1000 hectares & 4109274 & 4013773 & 3983481 \\
\hline
\end{tabular}


Les chiffres de la section des paiements au titre du premier pilier sont décomposés dans la feuille de calcul relative au soutien. Les dépenses d'exploitation comprennent tous les frais généraux et les coûts directs de l'exploitation. Le total des actifs agricoles comprend la valeur de l'exploitation, ainsi que les investissements dans les machines, animaux et bâtiments et les investissements dans l'entretien des terres. Le total des dettes agricoles inclut les emprunts restants et les nouveaux emprunts contractés dans l'année. Certains paiements du second pilier figurant dans la zone relative au premier pilier, celleci doit donc être considérée comme fournissant l'ensemble des paiements.

Italie

\begin{tabular}{|c|c|c|c|c|}
\hline Année & Unité & 2004 & 2006 & 2007 \\
\hline Exploitations couvertes & Nombre & 663908 & 682469 & 749355 \\
\hline $\begin{array}{l}\text { Unités de travail annuel (équivalent } \\
\text { temps plein) }\end{array}$ & UTA & 919807 & 953737 & 1021349 \\
\hline Superficie agricole utilisée & 1000 hectares & 10942 & 11817 & 11475 \\
\hline \multicolumn{5}{|l|}{ Pays-Bas } \\
\hline Année & Unité & 2004 & 2006 & 2007 \\
\hline Exploitations couvertes & Nombre & 64489 & 60440 & 58779 \\
\hline $\begin{array}{l}\text { Unités de travail annuel (équivalent } \\
\text { temps plein) }\end{array}$ & UTA & 157818 & 136603 & 140660 \\
\hline Superficie agricole utilisée & 1000 hectares & 1959 & 1942 & 1944 \\
\hline \multicolumn{5}{|l|}{ Espagne } \\
\hline Année & Unité & 2004 & 2006 & 2007 \\
\hline Exploitations couvertes & Nombre & 621374 & 606159 & 661514 \\
\hline $\begin{array}{l}\text { Unités de travail annuel (équivalent } \\
\text { temps plein) }\end{array}$ & UTA & 683511 & 606159 & 661514 \\
\hline Superficie agricole utilisée & 1000 hectares & 15907 & 15578 & 17067 \\
\hline
\end{tabular}

\section{Enquête RICA de l'Union européenne}

\section{UE27}

\begin{tabular}{|c|c|c|c|c|}
\hline Année & Unité & 2004 & 2006 & 2007 \\
\hline Exploitations couvertes & Nombre & 4104674 & 4116485 & 5071901 \\
\hline $\begin{array}{l}\text { Unités de travail annuel (équivalent } \\
\text { temps plein) }\end{array}$ & UTA & 6749410 & 6632492 & 8698236 \\
\hline Superficie agricole utilisée & 1000 hectares & 137066 & 141902 & 155190 \\
\hline \multicolumn{5}{|l|}{ UE15 } \\
\hline Année & Unité & 2004 & 2006 & 2007 \\
\hline Exploitations couvertes & Nombre & 3134746 & 3123446 & 3127987 \\
\hline $\begin{array}{l}\text { Unités de travail annuel (équivalent } \\
\text { temps plein) }\end{array}$ & UTA & 4828977 & 4697282 & 4779387 \\
\hline Superficie agricole utilisée & 1000 hectares & 111570 & 114452 & 115706 \\
\hline
\end{tabular}


UE10

\begin{tabular}{|c|c|c|c|c|}
\hline Année & Unité & 2004 & 2006 & 2007 \\
\hline Exploitations couvertes & Nombre & 969928 & 993038 & 992836 \\
\hline $\begin{array}{l}\text { Unités de travail annuel (équivalent } \\
\text { temps plein) }\end{array}$ & UTA & 1920433 & 1935210 & 1907842 \\
\hline Superficie agricole utilisée & 1000 hectares & 25496 & 27451 & 28033 \\
\hline \multicolumn{5}{|l|}{ UE2 } \\
\hline Année & Unité & 2004 & 2006 & 2007 \\
\hline Exploitations couvertes & Nombre & & & 312821 \\
\hline $\begin{array}{l}\text { Unités de travail annuel (équivalent } \\
\text { temps plein) }\end{array}$ & UTA & & & 691468 \\
\hline Superficie agricole utilisée & 1000 hectares & & & 7471 \\
\hline
\end{tabular}

Le RICA est une enquête par échantillonnage: les résultats de différentes exploitations sont utilisés pour représenter l'ensemble du secteur, à l'aide de coefficients de pondération. Les poids utilisés dans l'enquête RICA de l'Union européenne diffèrent de ceux des enquêtes RICA nationales. Le RICA est avant tout conçu pour représenter la situation économique des exploitations qui dépassent un seuil économique minimal. La couverture est proche de $90 \%$ de la marge brute standard, mais pas dans tous les États membres. Le RICA n'a pas été conçu pour être nécessairement représentatif de toutes les dimensions incluses dans la demande de données. En particulier, la définition des types d'exploitations utilisée dans la demande n'est normalement pas employée dans le RICA. Les exploitations de l'échantillon ont été réparties autant que possible selon les types d'exploitations agricoles proposés dans la demande de données. De plus, cet échantillon n'est pas conçu pour refléter parfaitement la distribution du soutien aux agriculteurs.

La couverture de l'ensemble de données UE27 n'est pas la même entre 2004 et 2006 d'une part, et 2007 d'autre part. Les données de 2004 et 2006 couvrent 25 pays, tandis que celles de 2007 en comprennent 27.

Le RICA ne permet pas de distinguer totalement les paiements de l'Union européenne des paiements nationaux. C'est pourquoi les données relatives aux paiements directs incluent également les paiements nationaux. La troisième catégorie, celle des « autres paiements ", est restée vide. En outre, il n'est pas possible dans tous les cas de faire la part entre les paiements du premier pilier et ceux du second. 


\section{États-Unis - Agricultural Resource Management Survey (ARMS)}

La documentation relative à l'enquête ARMS est disponible à l'adresse www.ers.usda.gov/Data/ARMS/GlobalDocumentation.htm.

Les exploitations agricoles retenues pour le projet du Réseau de l'OCDE sur l'analyse au niveau de l'exploitation représentent $90 \%$ de la production agricole brute nationale.

La production agricole brute est définie comme les recettes brutes diminuées des versements des pouvoirs publics et des indemnités du système fédéral d'assurance récolte. Les recettes brutes sont la somme du revenu agricole brut (gross cash farm income dans la nomenclature ERS-ARMS), qui comprend les recettes des ventes de produits agricoles, les recettes de la rémunération de travaux contractuels, les recettes des loyers fonciers, et les revenus liés à l'activité agricole. Ces derniers comprennent les versements des pouvoirs publics, les indemnités du système fédéral d'assurance récolte, les revenus de travaux à façon, et les revenus d'activités non agricoles inséparables de ceux de l'exploitation. Le revenu des ménages agricoles combine les revenus hors exploitation de l'exploitant et le revenu agricole net de l'ensemble des membres du ménage.

ERS distingue trois catégories de régions: rurales, urbanisées sans métropole, et métropoles. Dans ce rapport, les régions rurales sont classées comme essentiellement rurales, les régions avec métropoles comme essentiellement urbaines, et les régions urbaines sans métropoles comme intermédiaires. 
Annexe C.

Tableaux de référence 
64 - DisTRIBUtion DU SOUTIEN ET DES REVENUS DANS L'AGRICULTURE

Tableau annexe C1. Part des $25 \%$ d'exploitations les plus grandes dans certains pays de l'OCDE, par composante du revenu, 2004,2006 et 2007

\begin{tabular}{|c|c|c|c|c|c|c|c|c|c|c|c|c|c|c|c|c|c|c|}
\hline \multirow{3}{*}{$\begin{array}{l}\text { Pays } \\
\text { Quartile } \\
\text { Année }\end{array}$} & \multicolumn{3}{|c|}{ Canada } & \multicolumn{3}{|c|}{ États-Unis } & \multicolumn{3}{|c|}{ UE27 } & \multicolumn{3}{|c|}{ UE15 } & \multicolumn{3}{|c|}{ UE10 } & \multicolumn{3}{|c|}{ UE2 } \\
\hline & \multicolumn{3}{|c|}{$\begin{array}{l}\text { Part du 4ème } \\
\text { quartile en \% }\end{array}$} & \multicolumn{3}{|c|}{$\begin{array}{l}\text { Part du 4ème } \\
\text { quartile en \% }\end{array}$} & \multicolumn{3}{|c|}{$\begin{array}{l}\text { Part du 4ème } \\
\text { quartile en \% }\end{array}$} & \multicolumn{3}{|c|}{$\begin{array}{l}\text { Part du 4ème } \\
\text { quartile en \% }\end{array}$} & \multicolumn{3}{|c|}{$\begin{array}{l}\text { Part du 4ème } \\
\text { quartile en \% }\end{array}$} & \multicolumn{3}{|c|}{$\begin{array}{l}\text { Part du 4ème } \\
\text { quartile en \% }\end{array}$} \\
\hline & 2004 & 2006 & 2007 & 2004 & 2006 & 2007 & 2004 & 2006 & 2007 & 2004 & 2006 & 2007 & 2004 & 2006 & 2007 & 2004 & 2006 & 2007 \\
\hline Exploitations couvertes & 25 & 25 & 25 & 25 & 25 & 25 & 25 & 25 & 25 & 25 & 25 & 25 & 25 & 25 & 25 & n.a. & n.a. & 25 \\
\hline Unité de travail (éq. temps plein) & n.a. & n.a. & n.a. & n.a. & n.a. & n.a. & 42 & 42 & 40 & 43 & 43 & 44 & 45 & 45 & 45 & n.a. & n.a. & 39 \\
\hline Superficie agricole utilisée & 38 & 42 & 44 & 51 & 45 & 44 & 63 & 64 & 69 & 60 & 60 & 60 & 68 & 67 & 68 & n.a. & n.a. & 74 \\
\hline \multicolumn{19}{|l|}{ Composantes du revenu } \\
\hline Production agricole brute ${ }^{1}$ & 69 & 70 & 70 & 72 & 72 & 72 & 79 & 80 & 82 & 77 & 78 & 78 & 79 & 77 & 77 & n.a. & n.a. & 71 \\
\hline Soutien total & 52 & 55 & 58 & 65 & 61 & 65 & 75 & 71 & 74 & 70 & 68 & 65 & 75 & 69 & 70 & n.a. & n.a. & 76 \\
\hline Tous paiements $^{2}$ & 48 & 46 & 53 & 53 & 54 & 50 & 66 & 66 & 71 & 60 & 62 & 62 & 68 & 65 & 66 & n.a. & n.a. & 79 \\
\hline Soutien des prix du marché ${ }^{3}$ & 58 & 66 & 64 & 77 & 78 & 79 & 85 & 82 & 81 & 83 & 80 & 74 & 81 & 77 & 80 & n.a. & n.a. & 69 \\
\hline Recettes brutes ${ }^{4}$ & 67 & 68 & 68 & 71 & 71 & 69 & 77 & 78 & 81 & 74 & 76 & 76 & 77 & 75 & 75 & n.a. & n.a. & 72 \\
\hline Dépenses d'exploitation & 68 & 70 & 70 & 70 & 70 & 69 & 82 & 82 & 85 & 80 & 81 & 81 & 82 & 80 & 80 & n.a. & n.a. & 73 \\
\hline Revenu net d'exploitation ${ }^{5}$ & 59 & 58 & 60 & 74 & 73 & 69 & 69 & 70 & 74 & 66 & 67 & 68 & 68 & 65 & 68 & n.a. & n.a. & 71 \\
\hline Amortissements $^{6}$ & 55 & 58 & 59 & 59 & 60 & 59 & 72 & 72 & 76 & 70 & 71 & 71 & 63 & 65 & 65 & n.a. & n.a. & 56 \\
\hline Revenu agricole $^{7}$ & 65 & 57 & 62 & 78 & 79 & 72 & 67 & 68 & 73 & 64 & 66 & 67 & 72 & 65 & 70 & n.a. & n.a. & 75 \\
\hline Revenu non agricole ${ }^{8}$ & 15 & 16 & 17 & 26 & 24 & 20 & n.a. & n.a. & n.a. & n.a. & n.a. & n.a. & n.a. & n.a. & n.a. & n.a. & n.a. & n.a. \\
\hline Revenu des ménages agricoles ${ }^{9}$ & 39 & 33 & 39 & 62 & 68 & 58 & n.a. & n.a. & n.a. & n.a. & n.a. & n.a. & n.a. & n.a. & n.a. & n.a. & n.a. & n.a. \\
\hline Total des actifs agricoles ${ }^{11}$ & 49 & 50 & 50 & 49 & 47 & 48 & 64 & 64 & 68 & 61 & 60 & 60 & 63 & 62 & 62 & n.a. & n.a. & 55 \\
\hline Total des dettes agricoles ${ }^{12}$ & 59 & 58 & 61 & 58 & 61 & 60 & 89 & 90 & 93 & 87 & 88 & 88 & 88 & 88 & 88 & n.a. & n.a. & 93 \\
\hline Capitaux propres agricoles ${ }^{13}$ & 46 & 48 & 47 & 48 & 45 & 47 & 60 & 59 & 64 & 56 & 55 & 55 & 59 & 58 & 58 & n.a. & n.a. & 53 \\
\hline
\end{tabular}




\begin{tabular}{|c|c|c|c|c|c|c|c|c|c|c|c|c|c|c|c|c|c|c|}
\hline \multirow{3}{*}{$\begin{array}{l}\text { Pays } \\
\text { Quartile } \\
\text { Année }\end{array}$} & \multirow{2}{*}{\multicolumn{3}{|c|}{$\begin{array}{c}\text { Danemark } \\
\text { Part du 4ème } \\
\text { quartile en \% }\end{array}$}} & \multirow{2}{*}{\multicolumn{3}{|c|}{$\begin{array}{c}\text { Angleterre } \\
\text { Part du 4ème } \\
\text { quartile en \% }\end{array}$}} & \multirow{2}{*}{\multicolumn{3}{|c|}{$\begin{array}{c}\text { Espagne } \\
\text { Part du 4ème } \\
\text { quartile en \% }\end{array}$}} & \multirow{2}{*}{\multicolumn{3}{|c|}{$\begin{array}{c}\text { Estonie } \\
\text { Part du 4ème } \\
\text { quartile en \% }\end{array}$}} & \multirow{2}{*}{\multicolumn{3}{|c|}{$\begin{array}{c}\text { Flandre belge } \\
\text { Part du 4ème } \\
\text { quartile en \% }\end{array}$}} & \multirow{2}{*}{\multicolumn{3}{|c|}{$\begin{array}{c}\text { Pays-Bas } \\
\text { Part du 4ème } \\
\text { quartile en \% }\end{array}$}} \\
\hline & & & & & & & & & & & & & & & & & & \\
\hline & 2004 & 2006 & 2007 & 2004 & 2006 & 2007 & 2004 & 2006 & 2007 & 2004 & 2006 & 2007 & 2004 & 2006 & 2007 & 2004 & 2006 & 2007 \\
\hline Exploitations couvertes & 25 & 25 & 25 & 25 & 25 & 25 & 25 & 25 & 25 & 25 & 25 & 25 & 25 & 25 & 25 & 25 & 25 & 25 \\
\hline Unité de travail (éq. temps plein) & 39 & 44 & 44 & 54 & 57 & 56 & 32 & 33 & 40 & 62 & 59 & 56 & 38 & 41 & 41 & 49 & 46 & 47 \\
\hline Superficie agricole utilisée & 39 & 39 & 39 & 42 & 47 & 46 & 60 & 61 & 62 & 67 & 67 & 68 & 28 & 30 & 32 & 39 & 39 & 40 \\
\hline \multicolumn{19}{|l|}{ Composantes du revenu } \\
\hline Production agricole brute ${ }^{1}$ & 46 & 46 & 45 & 70 & 69 & 68 & 55 & 57 & 63 & 85 & 85 & 86 & 57 & 58 & 58 & 57 & 58 & 59 \\
\hline Soutien total & 44 & 40 & 39 & 51 & 51 & 52 & n.a. & n.a. & n.a. & 79 & 72 & 68 & 39 & 37 & 41 & 48 & 48 & 51 \\
\hline Tous paiements ${ }^{2}$ & 37 & 36 & 37 & 45 & 47 & 45 & 55 & 55 & 60 & 75 & 68 & 69 & 27 & 30 & 33 & 36 & 42 & 46 \\
\hline Soutien des prix du marché ${ }^{3}$ & 48 & 43 & 41 & 59 & 56 & 58 & n.a. & n.a. & n.a. & 86 & 80 & 67 & 43 & 41 & 48 & 51 & 52 & 55 \\
\hline Recettes brutes ${ }^{4}$ & 45 & 45 & 44 & 64 & 66 & 65 & 55 & 56 & 62 & 83 & 82 & 83 & 55 & 56 & 56 & 56 & 58 & 59 \\
\hline Dépenses d'exploitation & 47 & 47 & 46 & 66 & 67 & 67 & 58 & 60 & 66 & 85 & 85 & 84 & 58 & 60 & 60 & 56 & 57 & 59 \\
\hline Revenu net d'exploitation ${ }^{5}$ & 39 & 42 & 34 & 59 & 62 & 61 & 53 & 53 & 60 & 79 & 72 & 79 & 50 & 51 & 49 & 56 & 59 & 58 \\
\hline Amortissements ${ }^{6}$ & 45 & 44 & 44 & 56 & 61 & 59 & 49 & 45 & 55 & 80 & 83 & 83 & 48 & 51 & 51 & 56 & 57 & 58 \\
\hline Revenu agricole $^{7}$ & 34 & 40 & 17 & 61 & 63 & 62 & 53 & 54 & 60 & 79 & 66 & 77 & 51 & 51 & 48 & 56 & 61 & 59 \\
\hline Revenu non agricole ${ }^{8}$ & 28 & 30 & 27 & n.a. & n.a. & n.a. & n.a. & n.a. & n.a. & n.a. & n.a. & n.a. & n.a. & n.a. & n.a. & 24 & 20 & 21 \\
\hline Revenu des ménages agricoles ${ }^{9}$ & 33 & 38 & 21 & n.a. & n.a. & n.a. & n.a. & n.a. & n.a. & n.a. & n.a. & n.a. & n.a. & n.a. & n.a. & 47 & 50 & 48 \\
\hline Total des actifs agricoles ${ }^{11}$ & n.a. & n.a. & n.a. & 45 & 47 & 47 & 45 & 45 & 46 & 76 & 70 & 71 & 41 & 43 & 45 & 46 & 45 & 46 \\
\hline Total des dettes agricoles ${ }^{12}$ & n.a. & n.a. & n.a. & 59 & 61 & 62 & 58 & 59 & 67 & 89 & 88 & 86 & 51 & 51 & 54 & 53 & 55 & 55 \\
\hline Capitaux propres agricoles ${ }^{13}$ & n.a. & n.a. & n.a. & 43 & 45 & 45 & 45 & 45 & 45 & 72 & 63 & 65 & 38 & 40 & 41 & 42 & 40 & 40 \\
\hline
\end{tabular}


66 - DISTRIBUTION DU SOUTIEN ET DES REVENUS DANS L'AGRICULTURE

\begin{tabular}{|c|c|c|c|c|c|c|c|c|c|c|c|c|c|c|c|}
\hline \multirow{3}{*}{$\begin{array}{l}\text { Pays } \\
\text { Quartile } \\
\text { Année }\end{array}$} & \multirow{2}{*}{\multicolumn{3}{|c|}{$\begin{array}{c}\text { Finlande } \\
\text { Part du 4ème } \\
\text { quartile en \% }\end{array}$}} & \multirow{2}{*}{\multicolumn{3}{|c|}{$\begin{array}{c}\text { France } \\
\text { Part du 4ème } \\
\text { quartile en \% }\end{array}$}} & \multirow{2}{*}{\multicolumn{3}{|c|}{$\begin{array}{c}\text { Allemagne } \\
\text { Part du 4ème } \\
\text { quartile en \% }\end{array}$}} & \multirow{2}{*}{\multicolumn{3}{|c|}{$\begin{array}{c}\text { Irlande } \\
\text { Part du 4ème } \\
\text { quartile en } \%\end{array}$}} & \multicolumn{3}{|c|}{ Italie } \\
\hline & & & & & & & & & & & & & $\begin{array}{l}\text { Part } \\
\text { qua }\end{array}$ & $\begin{array}{l}t \text { du 4è } \\
\text { ittile en }\end{array}$ & \\
\hline & 2004 & 2006 & 2007 & 2004 & 2006 & 2007 & 2004 & 2006 & 2007 & 2004 & 2006 & 2007 & 2004 & 2006 & 2007 \\
\hline Exploitations couvertes & 25 & 25 & 25 & 25 & 25 & 25 & 25 & 25 & 25 & 25 & 25 & 25 & 25 & 25 & 25 \\
\hline Unité de travail (éq. temps plein) & 41 & 43 & 42 & 45 & 45 & 44 & 50 & 49 & 49 & n.a. & n.a. & n.a. & 31 & 32 & 28 \\
\hline Superficie agricole utilisée & 33 & 34 & 37 & 36 & 36 & 38 & 57 & 57 & 57 & 43 & 45 & 44 & 35 & 33 & 26 \\
\hline \multicolumn{16}{|l|}{ Composantes du revenu } \\
\hline Production agricole brute $^{1}$ & 60 & 62 & 61 & 57 & 58 & 58 & 65 & 65 & 65 & 73 & 72 & 75 & 56 & 59 & 46 \\
\hline Soutien total & n.a. & n.a. & n.a. & n.a. & n.a. & n.a. & 57 & 55 & 55 & 50 & 51 & 51 & 43 & 45 & 35 \\
\hline Tous paiements $^{2}$ & 40 & 42 & 44 & 36 & 37 & 39 & 56 & 54 & 54 & 40 & 45 & 45 & 40 & 43 & 33 \\
\hline Soutien des prix du marché ${ }^{3}$ & n.a. & n.a. & n.a. & n.a. & n.a. & n.a. & 58 & 56 & 55 & 73 & 72 & 75 & 51 & 52 & 38 \\
\hline Recettes brutes ${ }^{4}$ & 52 & 54 & 54 & 53 & 54 & 55 & 64 & 63 & 63 & 63 & 63 & 66 & 55 & 57 & 45 \\
\hline Dépenses d'exploitation & 54 & 55 & 57 & 55 & 56 & 57 & 67 & 67 & 67 & 63 & 64 & 64 & 54 & 55 & 44 \\
\hline Revenu net d'exploitation ${ }^{5}$ & 49 & 52 & 51 & 49 & 51 & 52 & 56 & 55 & 55 & 63 & 62 & 68 & 66 & 84 & 53 \\
\hline Amortissements ${ }^{6}$ & 47 & 49 & 50 & 49 & 48 & 49 & 55 & 54 & 55 & 60 & 60 & 61 & 42 & 43 & 34 \\
\hline Revenu agricole $^{7}$ & 50 & 55 & 51 & 49 & 52 & 54 & 56 & 55 & 55 & 64 & 63 & 69 & 1 & -56 & -126 \\
\hline Revenu non agricole $^{8}$ & n.a. & n.a. & n.a. & n.a. & n.a. & n.a. & n.a. & n.a. & n.a. & n.a. & n.a. & n.a. & n.a. & n.a. & n.a. \\
\hline Revenu des ménages agricoles ${ }^{9}$ & n.a. & n.a. & n.a. & n.a. & n.a. & n.a. & n.a. & n.a. & n.a. & n.a. & n.a. & n.a. & n.a. & n.a. & n.a. \\
\hline Total des actifs agricoles ${ }^{11}$ & 43 & 44 & 45 & n.a. & n.a. & n.a. & 42 & 41 & 42 & 50 & 51 & 51 & n.a. & n.a. & n.a. \\
\hline Total des dettes agricoles ${ }^{12}$ & 52 & 54 & 54 & n.a. & n.a. & n.a. & 62 & 61 & 63 & 79 & 75 & 71 & n.a. & n.a. & n.a. \\
\hline Capitaux propres agricoles ${ }^{13}$ & 40 & 40 & 42 & n.a. & n.a. & n.a. & 38 & 37 & 38 & 49 & 50 & 51 & n.a. & n.a. & n.a. \\
\hline
\end{tabular}




\section{Notes des tableaux annexe C1 et C2.}

n.a. : non disponible.

Les exploitations incluses représentent au total $90 \%$ de la production agricole nationale.

Les limites des quartiles sont établies à partir de la production agricole brute.

I. Le chiffre d'affaires brut des exploitations représentant $90 \%$ de la production agricole brute nationale est supérieur à 100000 CAD. Le revenu non agricole est susceptible d'être surévalué, car il peut inclure le revenu agricole transféré sous forme de salaires aux membres de la famille.

II. Les valeurs extrêmes n'ont pas été éliminées du revenu non agricole. Toutes les données sur les exploitations individuelles ont été pondérées en fonction des coefficients de pondération de l'année de référence. Nombre d'exploitations incluses dans la population (exploitations couvertes, à partir d'échantillons d'environ 11500 exploitations chaque année). Autres variables : moyennes pondérées des agrégats de référence.

1. Inclut les recettes tirées des ventes de produits et de services agricoles tels que le travail à façon ou la mise en location de terres, de quotas, de bâtiments ou de machines, entre autres.

2. Inclut les transferts budgétaires aux agriculteurs résultant de la politique agricole et des paiements des systèmes d'assurance, des programmes de conservation des terres aux États-Unis et des premier et second piliers de la Politique agricole commune dans l'Union européenne.

3. Le soutien des prix du marché (SPM) est calculé en appliquant le ratio SPM de chaque produit de base, lorsqu'il existe dans la base de données des ESP de I'OCDE, aux recettes de l'exploitation tirées de ce produit. Le ratio SPM moyen de l'ensemble des produits de la base ESP est utilisé pour les recettes provenant de produits pour lesquels il n'existe pas de ratio SPM.

4. Comprend la production agricole brute et l'ensemble des paiements.

5. Différence entre les recettes brutes et les dépenses d'exploitation. Correspond au revenu agricole avant amortissements.

6. Comprend l'estimation nationale de l'amortissement économique ou l'estimation de la déduction fiscale pour amortissement lorsque celle-ci est disponible.

7. Correspond au revenu net d'exploitation diminué des dotations aux amortissements.

8. Inclut le revenu non agricole des exploitants et des autres membres de leur ménage. En sont exclus les revenus non agricoles des ménages dirigeant une exploitation constituée en société.

9. Inclut les revenus agricole et non agricole.

Source : tableaux transmis par les participants. 
$\mathbf{6 8}$ - Distribution DU SOUTIEN ET DES REVENUS DANS L'AGRICULTURE

Tableau annexe C2. Part des $25 \%$ d'exploitations les plus petites dans certains pays de l'OCDE, par composante du revenu, 2004,2006 et 2007

\begin{tabular}{|c|c|c|c|c|c|c|c|c|c|c|c|c|c|c|c|c|c|c|}
\hline $\begin{array}{l}\text { Pays } \\
\text { Quartile } \\
\text { Année }\end{array}$ & \multicolumn{3}{|c|}{$\begin{array}{c}\text { Canada } \\
\text { Part du 1er } \\
\text { quartile en } \%\end{array}$} & \multicolumn{3}{|c|}{$\begin{array}{c}\text { États-Unis } \\
\text { Part du 1er } \\
\text { quartile en \% }\end{array}$} & \multicolumn{3}{|c|}{ UE27 } & \multicolumn{3}{|c|}{ UE15 } & \multicolumn{3}{|c|}{ UE10 } & \multicolumn{3}{|c|}{ UE2 } \\
\hline Exploitations couvertes & 25 & 25 & 25 & 25 & 25 & 25 & 25 & 25 & 25 & 25 & 25 & 25 & 25 & 25 & 25 & n.a. & n.a. & 25 \\
\hline Unité de travail (éq. temps plein) & n.a. & n.a. & n.a. & n.a. & n.a. & n.a. & 16 & 16 & 20 & 15 & 14 & 15 & 15 & 16 & 16 & n.a. & n.a. & 17 \\
\hline Superficie agricole utilisée & 15 & 13 & 13 & 11 & 14 & 14 & 6 & 7 & 5 & 6 & 6 & 6 & 7 & 8 & 7 & n.a. & n.a. & 6 \\
\hline
\end{tabular}

\begin{tabular}{|c|c|c|c|c|c|c|c|c|c|c|c|c|c|c|c|c|c|c|}
\hline \multicolumn{19}{|l|}{ Composantes du revenu } \\
\hline Production agricole brute ${ }^{1}$ & 6 & 5 & 5 & 5 & 5 & 5 & 2 & 2 & 2 & 2 & 2 & 2 & 3 & 4 & 4 & n.a. & n.a. & 5 \\
\hline Soutien total & 12 & 11 & 9 & 6 & 7 & 5 & 4 & 5 & 3 & 4 & 5 & 5 & 5 & 7 & 7 & n.a. & n.a. & 5 \\
\hline Tous paiements $^{2}$ & 19 & 18 & 14 & 8 & 9 & 8 & 6 & 7 & 4 & 7 & 7 & 6 & 8 & 9 & 9 & n.a. & n.a. & 5 \\
\hline Soutien des prix du marché ${ }^{3}$ & 2 & 2 & 2 & 3 & 4 & 2 & 1 & 1 & 2 & 1 & 1 & 2 & 3 & 4 & 3 & n.a. & n.a. & 6 \\
\hline Recettes brutes $^{4}$ & 7 & 6 & 6 & 5 & 6 & 5 & 3 & 3 & 2 & 3 & 3 & 3 & 4 & 5 & 5 & n.a. & n.a. & 5 \\
\hline Dépenses d'exploitation & 7 & 6 & 6 & 6 & 6 & 6 & 2 & 2 & 2 & 2 & 2 & 2 & 3 & 4 & 4 & n.a. & n.a. & 7 \\
\hline Revenu agricole $^{7}$ & 5 & 7 & 5 & 3 & 4 & 2 & 3 & 3 & 2 & 4 & 4 & 4 & 2 & 5 & 4 & n.a. & n.a. & -1 \\
\hline Revenu non agricole 8 & 31 & 33 & 32 & 26 & 26 & 26 & n.a. & n.a. & n.a. & n.a. & n.a. & n.a. & n.a. & n.a. & n.a. & n.a. & n.a. & n.a. \\
\hline Revenu des ménages agricoles ${ }^{9}$ & 19 & 22 & 18 & 10 & 13 & 9 & n.a. & n.a. & n.a. & n.a. & n.a. & n.a. & n.a. & n.a. & n.a. & n.a. & n.a. & n.a. \\
\hline Total des actifs agricoles ${ }^{11}$ & 11 & 12 & 12 & 10 & 13 & 9 & 6 & 6 & 5 & 7 & 7 & 8 & 9 & 10 & 9 & n.a. & n.a. & 10 \\
\hline
\end{tabular}




\begin{tabular}{|c|c|c|c|c|c|c|c|c|c|c|c|c|c|c|c|c|c|c|}
\hline \multirow{2}{*}{$\begin{array}{l}\text { Pays } \\
\text { Quartile } \\
\text { Année }\end{array}$} & \multirow{2}{*}{\multicolumn{3}{|c|}{$\begin{array}{c}\text { Danemark } \\
\text { Part du 1er } \\
\text { quartile en } \%\end{array}$}} & \multirow{2}{*}{\multicolumn{3}{|c|}{$\begin{array}{c}\text { Angleterre } \\
\text { Part du 1er } \\
\text { quartile en \% }\end{array}$}} & \multirow{2}{*}{\multicolumn{3}{|c|}{$\begin{array}{c}\text { Espagne } \\
\text { Part du 1er } \\
\text { quartile en } \%\end{array}$}} & \multirow{2}{*}{\multicolumn{3}{|c|}{$\begin{array}{c}\text { Estonie } \\
\text { Part du 1er } \\
\text { quartile en \% }\end{array}$}} & \multirow{2}{*}{\multicolumn{3}{|c|}{$\begin{array}{c}\text { Flandre belge } \\
\text { Part du 1er } \\
\text { quartile en } \%\end{array}$}} & \multirow{2}{*}{\multicolumn{3}{|c|}{$\begin{array}{c}\text { Pays-Bas } \\
\text { Part du 1er } \\
\text { quartile en \% }\end{array}$}} \\
\hline & & & & & & & & & & & & & & & & & & \\
\hline Exploitations couvertes & 25 & 25 & 25 & 25 & 25 & 25 & 25 & 25 & 25 & 25 & 25 & 25 & 25 & 25 & 25 & 25 & 25 & 25 \\
\hline Unité de travail (éq. temps plein) & 17 & 15 & 15 & 10 & 10 & 10 & 23 & 18 & 15 & 11 & 12 & 12 & 17 & 15 & 15 & 12 & 13 & 12 \\
\hline Superficie agricole utilisée & 15 & 16 & 16 & 14 & 13 & 13 & 6 & 6 & 7 & 9 & 9 & 9 & 17 & 18 & 18 & 14 & 14 & 14 \\
\hline \multicolumn{19}{|l|}{ Composantes du revenu } \\
\hline Production agricole brute $^{1}$ & 13 & 13 & 13 & 4 & 3 & 3 & 9 & 8 & 5 & 2 & 2 & 2 & 6 & 6 & 6 & 6 & 6 & 6 \\
\hline Soutien total & 13 & 14 & 13 & 9 & 10 & 10 & n.a. & n.a. & n.a. & 5 & 7 & 8 & 11 & 13 & 13 & 10 & 10 & 9 \\
\hline Tous paiements $^{2}$ & 15 & 18 & 16 & 11 & 12 & 12 & 12 & 13 & 9 & 7 & 9 & 9 & 19 & 16 & 15 & 18 & 14 & 11 \\
\hline Soutien des prix du marché ${ }^{3}$ & 11 & 13 & 11 & 6 & 8 & 7 & n.a. & n.a. & n.a. & 2 & 2 & 6 & 9 & 10 & 11 & 8 & 8 & 8 \\
\hline Recettes brutes ${ }^{4}$ & 13 & 14 & 13 & 5 & 5 & 4 & 10 & 9 & 6 & 3 & 4 & 4 & 7 & 6 & 6 & 7 & 6 & 6 \\
\hline Revenu agricole ${ }^{7}$ & 15 & 18 & 20 & 4 & 3 & 2 & 11 & 9 & 5 & 5 & 10 & 5 & 7 & 6 & 6 & 3 & 2 & 4 \\
\hline Revenu non agricole 8 & 26 & 19 & 24 & n.a. & n.a. & n.a. & n.a. & n.a. & n.a. & n.a. & n.a. & n.a. & n.a. & n.a. & n.a. & 30 & 30 & 35 \\
\hline Revenu des ménages agricoles ${ }^{9}$ & 18 & 18 & 21 & n.a. & n.a. & n.a. & n.a. & n.a. & n.a. & n.a. & n.a. & n.a. & n.a. & n.a. & n.a. & 11 & 9 & 12 \\
\hline Total des actifs agricoles ${ }^{11}$ & n.a. & n.a. & n.a. & 12 & 12 & 13 & 15 & 14 & 15 & 7 & 7 & 8 & 13 & 12 & 11 & 11 & 11 & 11 \\
\hline Total des dettes agricoles ${ }^{12}$ & n.a. & n.a. & n.a. & 6 & 6 & 6 & 13 & 8 & 6 & 1 & 1 & 3 & 9 & 8 & 7 & 7 & 7 & 7 \\
\hline Capitaux propres agricoles ${ }^{13}$ & n.a. & n.a. & n.a. & 13 & 13 & 14 & 15 & 14 & 15 & 8 & 9 & 10 & 14 & 14 & 12 & 12 & 14 & 14 \\
\hline
\end{tabular}




\begin{tabular}{|c|c|c|c|c|c|c|c|c|c|c|c|c|c|c|c|}
\hline \multirow{3}{*}{$\begin{array}{l}\text { Pays } \\
\text { Quartile } \\
\text { Année }\end{array}$} & \multirow{2}{*}{\multicolumn{3}{|c|}{$\begin{array}{c}\text { Finlande } \\
\text { Part du 1er } \\
\text { quartile en } \%\end{array}$}} & \multirow{2}{*}{\multicolumn{3}{|c|}{$\begin{array}{c}\text { France } \\
\text { Part du 1er } \\
\text { quartile en } \%\end{array}$}} & \multirow{2}{*}{\multicolumn{3}{|c|}{$\begin{array}{c}\text { Allemagne } \\
\text { Part du 1er } \\
\text { quartile en \% }\end{array}$}} & \multirow{2}{*}{\multicolumn{3}{|c|}{$\begin{array}{c}\text { Irlande } \\
\text { Part du 1er } \\
\text { quartile en } \%\end{array}$}} & \multicolumn{3}{|c|}{ Italie } \\
\hline & & & & & & & & & & & & & & $\begin{array}{l}\text { art du } 1 \\
\text { artile en }\end{array}$ & \\
\hline & 2004 & 2006 & 2007 & 2004 & 2006 & 2007 & 2004 & 2006 & 2007 & 2004 & 2006 & 2007 & 2004 & 2006 & 2007 \\
\hline Exploitations couvertes & 25 & 25 & 25 & 25 & 25 & 25 & 25 & 25 & 25 & 25 & 25 & 25 & 25 & 25 & 25 \\
\hline Unité de travail (éq. temps plein) & 10 & 10 & 10 & 15 & 15 & 15 & 12 & 12 & 12 & n.a. & n.a. & n.a. & 21 & 21 & 24 \\
\hline Superficie agricole utilisée & 20 & 17 & 16 & 16 & 16 & 15 & 9 & 9 & 9 & 12 & 12 & 12 & 18 & 21 & 28 \\
\hline \multicolumn{16}{|l|}{ Composantes du revenu } \\
\hline Production agricole brute ${ }^{1}$ & 4 & 4 & 4 & 7 & 6 & 6 & 4 & 4 & 4 & 3 & 3 & 2 & 8 & 8 & 13 \\
\hline Soutien total & n.a. & n.a. & n.a. & n.a. & n.a. & n.a. & 7 & 8 & 8 & 9 & 10 & 9 & 16 & 18 & 22 \\
\hline Tous paiements ${ }^{2}$ & 15 & 13 & 13 & 17 & 16 & 15 & 10 & 10 & 9 & 13 & 11 & 11 & 18 & 19 & 25 \\
\hline Soutien des prix du marché ${ }^{3}$ & n.a. & n.a. & n.a. & n.a. & n.a. & n.a. & 4 & 5 & 6 & 3 & 3 & 2 & 9 & 10 & 18 \\
\hline Recettes brutes ${ }^{4}$ & 9 & 8 & 8 & 8 & 8 & 7 & 5 & 5 & 5 & 5 & 6 & 5 & 9 & 10 & 14 \\
\hline Dépenses d'exploitation & 9 & 9 & 8 & 8 & 8 & 7 & 5 & 5 & 5 & 6 & 5 & 5 & 10 & 11 & 15 \\
\hline Revenu net d'exploitation ${ }^{5}$ & 7 & 5 & 7 & 9 & 8 & 8 & 4 & 4 & 5 & 5 & 6 & 5 & -3 & -4 & 8 \\
\hline Amortissements $^{6}$ & 13 & 11 & 11 & 9 & 9 & 9 & 8 & 8 & 7 & 7 & 6 & 5 & 14 & 15 & 20 \\
\hline Revenu agricole $^{7}$ & 1 & 0 & 4 & 10 & 8 & 7 & 1 & 2 & 4 & 5 & 7 & 5 & 42 & 61 & 129 \\
\hline Revenu non agricole ${ }^{8}$ & n.a. & n.a. & n.a. & n.a. & n.a. & n.a. & n.a. & n.a. & n.a. & n.a. & n.a. & n.a. & n.a. & n.a. & n.a. \\
\hline Revenu des ménages agricoles ${ }^{9}$ & n.a. & n.a. & n.a. & n.a. & n.a. & n.a. & n.a. & n.a. & n.a. & n.a. & n.a. & n.a. & n.a. & n.a. & n.a. \\
\hline Total des actifs agricoles ${ }^{11}$ & 15 & 12 & 11 & n.a. & n.a. & n.a. & 13 & 13 & 13 & 9 & 9 & 8 & n.a. & n.a. & n.a. \\
\hline Total des dettes agricoles ${ }^{12}$ & 13 & 11 & 8 & n.a. & n.a. & n.a. & 7 & 6 & 5 & 2 & 1 & 2 & n.a. & n.a. & n.a. \\
\hline Capitaux propres agricoles ${ }^{13}$ & 15 & 13 & 13 & n.a. & n.a. & n.a. & 14 & 14 & 14 & 9 & 9 & 8 & n.a. & n.a. & n.a. \\
\hline
\end{tabular}

Notes identiques à celles du tableau $\mathrm{C} 1$.

Source : tableaux transmis par les participants. 
Tableau annexe C3. Ratio de la moyenne des exploitations regroupées par taille d'exploitation, sur la moyenne de l'ensemble des exploitations, certains pays de l'OCDE, 2004, 2006, 2007

\begin{tabular}{|c|c|c|c|c|c|c|c|c|c|c|c|c|c|}
\hline & \multicolumn{13}{|c|}{ Canada } \\
\hline & \multirow[t]{2}{*}{ Unité } & \multicolumn{3}{|c|}{$\begin{array}{l}\text { Moyenne de l'ensemble des } \\
\text { exploitations }\end{array}$} & \multicolumn{3}{|c|}{$\begin{array}{l}\text { Rapport entre le 1er quartile et } \\
\text { l'ensemble des exploitations }\end{array}$} & \multicolumn{3}{|c|}{$\begin{array}{l}\text { Rapport entre le 4ème quartile } \\
\text { et l'ensemble des exploitations }\end{array}$} & \multicolumn{3}{|c|}{$\begin{array}{l}\text { Rapport entre le 4ème et le 1er } \\
\text { quartiles }\end{array}$} \\
\hline & & 2004 & 2006 & 2007 & 2004 & 2006 & 2007 & 2004 & 2006 & 2007 & 2004 & 2006 & 2007 \\
\hline Unité de travail (éq. temps plein) & UTA par ferme & n.a. & n.a. & n.a. & n.a. & n.a. & n.a. & n.a. & n.a. & n.a. & n.a. & n.a. & n.a. \\
\hline Superficie agricole utilisée & Ha par ferme & 2007.1 & 2051.1 & 2350.1 & 0.6 & 0.5 & 0.5 & 1.5 & 1.7 & 1.8 & 2.5 & 3.1 & 3.3 \\
\hline Production agricole brute ${ }^{1}$ & EUR par ferme & 271356 & 337745 & 357666 & 0.2 & 0.2 & 0.2 & 2.7 & 2.8 & 2.8 & 12.1 & 13.5 & 13.4 \\
\hline Soutien total & EUR par ferme & 45136 & 61955 & 51722 & 0.5 & 0.4 & 0.3 & 2.1 & 2.2 & 2.3 & 4.5 & 5.2 & 6.7 \\
\hline Tous paiements $^{2}$ & EUR par ferme & 25681 & 32818 & 28282 & 0.7 & 0.7 & 0.6 & 1.9 & 1.8 & 2.1 & 2.6 & 2.5 & 3.7 \\
\hline Paiements du 1er pilier & EUR par ferme & n.a. & n.a. & n.a. & n.a. & n.a. & n.a. & n.a. & n.a. & n.a. & n.a. & n.a. & n.a. \\
\hline Paiements du 2ème pilier & EUR par ferme & n.a. & n.a. & n.a. & n.a. & n.a. & n.a. & n.a. & n.a. & n.a. & n.a. & n.a. & n.a. \\
\hline Autres paiements & EUR par ferme & 25681 & 32818 & 28282 & 0.7 & 0.7 & 0.6 & 1.9 & 1.8 & 2.1 & 2.6 & 2.5 & 3.7 \\
\hline Soutien des prix du marché ${ }^{3}$ & EUR par ferme & 19455 & 29137 & 23441 & 0.1 & 0.1 & 0.1 & 2.3 & 2.6 & 2.5 & 24.1 & 37.1 & 32.5 \\
\hline Recettes brutes ${ }^{4}$ & EUR par ferme & 297037 & 370563 & 385948 & 0.3 & 0.3 & 0.2 & 2.7 & 2.7 & 2.7 & 9.8 & 10.7 & 11.7 \\
\hline Dépenses d'exploitation & EUR par ferme & 253254 & 320881 & 328486 & 0.3 & 0.2 & 0.2 & 2.7 & 2.8 & 2.8 & 10.3 & 11.4 & 12.2 \\
\hline Revenu net d'exploitation ${ }^{5}$ & EUR par ferme & 43783 & 49682 & 57462 & 0.3 & 0.3 & 0.3 & 2.4 & 2.3 & 2.4 & 7.6 & 7.2 & 8.9 \\
\hline Amortissements ${ }^{6}$ & EUR par ferme & 27637 & 33530 & 33002 & 0.4 & 0.3 & 0.3 & 2.2 & 2.3 & 2.3 & 6.0 & 6.7 & 7.2 \\
\hline Revenu agricole $^{7}$ & EUR par ferme & 16146 & 16152 & 24460 & 0.2 & 0.3 & 0.2 & 2.6 & 2.3 & 2.5 & 13.0 & 8.6 & 12.8 \\
\hline Revenu non agricole ${ }^{8}$ & EUR par ferme & 17651 & 23131 & 23832 & 1.3 & 1.3 & 1.3 & 0.6 & 0.6 & 0.7 & 0.5 & 0.5 & 0.5 \\
\hline \multirow[t]{2}{*}{ Revenu des ménages agricoles ${ }^{9}$} & EUR par ferme & 33797 & 39283 & 48292 & 0.7 & 0.9 & 0.7 & 1.5 & 1.3 & 1.6 & 2.1 & 1.5 & 2.2 \\
\hline & EUR par ferme & & & & & & & & & & & & \\
\hline Total des actifs agricoles ${ }^{11}$ & EUR par ferme & 1082930 & 1323693 & 1522309 & 0.5 & 0.5 & 0.5 & 2.0 & 2.0 & 2.0 & 4.4 & 4.3 & 4.3 \\
\hline Total des dettes agricoles ${ }^{12}$ & EUR par ferme & 257165 & 284896 & 321481 & 0.3 & 0.3 & 0.3 & 2.3 & 2.3 & 2.4 & 7.8 & 7.4 & 8.9 \\
\hline \multirow[t]{4}{*}{ Capitaux propres agricoles ${ }^{13}$} & EUR par ferme & 825764 & 1038797 & 1200827 & 0.5 & 0.5 & 0.5 & 1.8 & 1.9 & 1.9 & 3.7 & 3.8 & 3.6 \\
\hline & & \multicolumn{12}{|c|}{ États-Unis } \\
\hline & Unité & \multicolumn{3}{|c|}{$\begin{array}{l}\text { Moyenne de l'ensemble des } \\
\text { exploitations }\end{array}$} & \multicolumn{3}{|c|}{$\begin{array}{l}\text { Rapport entre le 1er quartile et } \\
\text { l'ensemble des exploitations }\end{array}$} & \multicolumn{3}{|c|}{$\begin{array}{l}\text { Rapport entre le 4ème quartile } \\
\text { et l'ensemble des exploitations }\end{array}$} & \multicolumn{3}{|c|}{$\begin{array}{l}\text { Rapport entre le 4ème et le 1er } \\
\text { quartiles }\end{array}$} \\
\hline & & 2004 & 2006 & 2007 & 2004 & 2006 & 2007 & 2004 & 2006 & 2007 & 2004 & 2006 & 2007 \\
\hline Unité de travail (éq. temps plein) & UTA par ferme & n.a. & n.a. & n.a. & n.a. & n.a. & n.a. & n.a. & n.a. & n.a. & n.a. & n.a. & n.a. \\
\hline Superficie agricole utilisée & Ha par ferme & 639.9 & 594.6 & 610.4 & 0.4 & 0.5 & 0.5 & 2.0 & 1.8 & 1.8 & 4.6 & 3.3 & 3.2 \\
\hline Production agricole brute ${ }^{1}$ & EUR par ferme & 348124 & 360504 & 463648 & 0.2 & 0.2 & 0.2 & 2.9 & 2.9 & 2.9 & 14.0 & 13.3 & 14.2 \\
\hline Soutien total & EUR par ferme & 31298 & 23385 & 33763 & 0.2 & 0.3 & 0.2 & 2.6 & 2.4 & 2.6 & 11.6 & 8.4 & 12.6 \\
\hline Tous paiements $^{2}$ & EUR par ferme & 15647 & 16766 & 15542 & 0.3 & 0.3 & 0.3 & 2.1 & 2.2 & 2.0 & 6.4 & 6.3 & 5.8 \\
\hline Paiements du 1er pilier & EUR par ferme & n.a. & n.a. & n.a. & n.a. & n.a. & n.a. & n.a. & n.a. & n.a. & n.a. & n.a. & n.a. \\
\hline Paiements du 2ème pilier & EUR par ferme & n.a. & n.a. & n.a. & n.a. & n.a. & n.a. & n.a. & n.a. & n.a. & n.a. & n.a. & n.a. \\
\hline Autres paiements & EUR par ferme & n.a. & n.a. & n.a. & n.a. & n.a. & n.a. & n.a. & n.a. & n.a. & n.a. & n.a. & n.a. \\
\hline Soutien des prix du marché ${ }^{3}$ & EUR par ferme & 15651 & 6619 & 18221 & 0.1 & 0.1 & 0.1 & 3.1 & 3.1 & 3.2 & 26.9 & 21.6 & 33.1 \\
\hline Recettes brutes ${ }^{4}$ & EUR par ferme & 363771 & 377269 & 479190 & 0.2 & 0.2 & 0.2 & 2.9 & 2.8 & 2.8 & 13.4 & 12.8 & 13.4 \\
\hline Dépenses d'exploitation & EUR par ferme & 255660 & 287737 & 345777 & 0.2 & 0.2 & 0.2 & 2.8 & 2.8 & 2.8 & 12.5 & 12.4 & 12.1 \\
\hline Revenu net d'exploitation ${ }^{5}$ & EUR par ferme & 108111 & 89533 & 133413 & 0.2 & 0.2 & 0.1 & 2.9 & 2.9 & 2.8 & 16.1 & 14.4 & 18.7 \\
\hline Amortissements ${ }^{6}$ & EUR par ferme & 26826 & 26301 & 29778 & 0.3 & 0.3 & 0.3 & 2.4 & 2.4 & 2.3 & 7.4 & 7.7 & 7.3 \\
\hline Revenu agricole $^{7}$ & EUR par ferme & 81285 & 63231 & 103635 & 0.1 & 0.2 & 0.1 & 3.1 & 3.2 & 2.9 & 22.7 & 19.9 & 29.5 \\
\hline Revenu non agricole ${ }^{8}$ & EUR par ferme & 36734 & 43911 & 36620 & 1.0 & 1.0 & 1.0 & 1.0 & 1.0 & 0.8 & 1.0 & 1.0 & 0.8 \\
\hline \multirow[t]{2}{*}{ Revenu des ménages agricoles ${ }^{9}$} & EUR par ferme & 118019 & 107142 & 140255 & 0.4 & 0.5 & 0.3 & 2.5 & 2.7 & 2.3 & 5.9 & 5.3 & 6.8 \\
\hline & EUR par ferme & & & & & & & & & & & & \\
\hline Total des actifs agricoles ${ }^{11}$ & EUR par ferme & 1414034 & 1782284 & 1716536 & 0.5 & 0.7 & 0.5 & 2.0 & 1.9 & 1.9 & 3.9 & 2.8 & 3.5 \\
\hline Total des dettes agricoles ${ }^{12}$ & EUR par ferme & 186738 & 203525 & 211325 & 0.3 & 0.3 & 0.4 & 2.3 & 2.4 & 2.4 & 7.1 & 7.1 & 6.6 \\
\hline Capitaux propres agricoles ${ }^{13}$ & EUR par ferme & 1227295 & 1578758 & 1505212 & 0.5 & 0.7 & 0.6 & 1.9 & 1.8 & 1.9 & 3.6 & 2.5 & 3.3 \\
\hline
\end{tabular}




\begin{tabular}{|c|c|c|c|c|c|c|c|c|c|c|c|c|c|}
\hline & \multicolumn{13}{|c|}{$\begin{array}{rr}\text { UE27 } \\
\end{array}$} \\
\hline & \multirow[t]{2}{*}{ Unité } & \multicolumn{3}{|c|}{$\begin{array}{l}\text { Moyenne de l'ensemble des } \\
\text { exploitations }\end{array}$} & \multicolumn{3}{|c|}{$\begin{array}{l}\text { Rapport entre le 1er quartile et } \\
\text { I'ensemble des exploitations }\end{array}$} & \multicolumn{3}{|c|}{$\begin{array}{l}\text { Rapport entre le 4ème quartile } \\
\text { et l'ensemble des exploitations }\end{array}$} & \multicolumn{3}{|c|}{$\begin{array}{l}\text { Rapport entre le 4ème et le 1er } \\
\text { quartiles }\end{array}$} \\
\hline & & 2004 & 2006 & 2007 & 2004 & 2006 & 2007 & 2004 & 2006 & 2007 & 2004 & 2006 & 2007 \\
\hline Unité de travail (éq. temps plein) & UTA par ferme & 1.6 & 1.6 & 1.7 & 0.7 & 0.6 & 0.8 & 1.7 & 1.7 & 1.6 & 2.6 & 2.7 & 2.0 \\
\hline Superficie agricole utilisée & Ha par ferme & 33.4 & 34.5 & 30.6 & 0.3 & 0.3 & 0.2 & 2.5 & 2.5 & 2.7 & 10.0 & 9.4 & 13.8 \\
\hline Production agricole brute ${ }^{1}$ & EUR par ferme & 60178 & 62771 & 60346 & 0.1 & 0.1 & 0.1 & 3.2 & 3.2 & 3.3 & 35.0 & 36.4 & 43.3 \\
\hline Soutien total & EUR par ferme & 19330 & 18101 & 14025 & 0.1 & 0.2 & 0.1 & 3.0 & 2.9 & 3.0 & 20.6 & 14.6 & 21.2 \\
\hline Tous paiements ${ }^{2}$ & EUR par ferme & 10241 & 11864 & 9816 & 0.2 & 0.3 & 0.2 & 2.6 & 2.6 & 2.8 & 11.8 & 9.8 & 16.7 \\
\hline Paiements du 1er pilier & EUR par ferme & 8635 & 9736 & 8121 & 0.2 & 0.2 & 0.2 & 2.7 & 2.7 & 2.9 & 12.3 & 11.4 & 18.9 \\
\hline Paiements du 2ème pilier & EUR par ferme & 1606 & 2128 & 1695 & 0.2 & 0.4 & 0.2 & 2.3 & 2.2 & 2.5 & 9.5 & 5.5 & 9.9 \\
\hline Autres paiements & EUR par ferme & n.a. & n.a. & n.a. & n.a. & n.a. & n.a. & n.a. & n.a. & n.a. & n.a. & n.a. & n.a. \\
\hline Soutien des prix du marché ${ }^{3}$ & EUR par ferme & 9089 & 6238 & 4209 & 0.1 & 0.1 & 0.1 & 3.4 & 3.3 & 3.2 & 58.5 & 56.3 & 47.8 \\
\hline Recettes brutes ${ }^{4}$ & EUR par ferme & 70419 & 74635 & 70162 & 0.1 & 0.1 & 0.1 & 3.1 & 3.1 & 3.2 & 28.2 & 26.7 & 36.2 \\
\hline Dépenses d'exploitation & EUR par ferme & 44147 & 46238 & 42817 & 0.1 & 0.1 & 0.1 & 3.3 & 3.3 & 3.4 & 36.7 & 36.5 & 42.5 \\
\hline Revenu net d'exploitation ${ }^{5}$ & EUR par ferme & 26273 & 28396 & 27345 & 0.1 & 0.2 & 0.1 & 2.7 & 2.8 & 3.0 & 19.2 & 17.6 & 28.6 \\
\hline Amortissements ${ }^{6}$ & EUR par ferme & 8176 & 8831 & 7598 & 0.2 & 0.2 & 0.1 & 2.9 & 2.9 & 3.0 & 13.9 & 13.9 & 20.6 \\
\hline Revenu agricole ${ }^{7}$ & EUR par ferme & 18097 & 19565 & 19747 & 0.1 & 0.1 & 0.1 & 2.7 & 2.7 & 2.9 & 23.6 & 20.1 & 33.7 \\
\hline Revenu non agricole ${ }^{8}$ & EUR par ferme & n.a. & n.a. & n.a. & n.a. & n.a. & n.a. & n.a. & n.a. & n.a. & n.a. & n.a. & n.a. \\
\hline \multirow[t]{2}{*}{ Revenu des ménages agricoles ${ }^{9}$} & EUR par ferme & n.a. & n.a. & n.a. & n.a. & n.a. & n.a. & n.a. & n.a. & n.a. & n.a. & n.a. & n.a. \\
\hline & EUR par ferme & & & & & & & & & & & & \\
\hline Total des actifs agricoles ${ }^{11}$ & EUR par ferme & 267211 & 309967 & 275908 & 0.2 & 0.3 & 0.2 & 2.6 & 2.6 & 2.7 & 10.7 & 9.9 & 14.7 \\
\hline Total des dettes agricoles ${ }^{12}$ & EUR par ferme & 41196 & 45111 & 39110 & 0.0 & 0.0 & 0.0 & 3.6 & 3.6 & 3.7 & 116.1 & 142.7 & 288.5 \\
\hline \multirow[t]{4}{*}{ Capitaux propres agricoles ${ }^{13}$} & EUR par ferme & 226015 & 264856 & 236798 & 0.3 & 0.3 & 0.2 & 2.4 & 2.4 & 2.6 & 8.5 & 8.0 & 12.0 \\
\hline & \multicolumn{13}{|c|}{ Danemark } \\
\hline & Unité & Moyenn & $\begin{array}{l}\text { e de l'ensemb } \\
\text { exploitations }\end{array}$ & le des & $\begin{array}{l}\text { Rapport en } \\
\text { l'ensemble }\end{array}$ & $\begin{array}{l}\text { tre le } 1 \text { er } \\
\text { des exp }\end{array}$ & $\begin{array}{l}\text { uartile et } \\
\text { itations }\end{array}$ & $\begin{array}{l}\text { Rapport e } \\
\text { et l'ensem }\end{array}$ & $\begin{array}{l}\text { tre le 4èr } \\
\text { le des ex }\end{array}$ & $\begin{array}{l}\text { quartile } \\
\text { oitations }\end{array}$ & Rapport er & $\begin{array}{l}\text { re le 4èm } \\
\text { quartiles }\end{array}$ & et le 1er \\
\hline & & 2004 & 2006 & 2007 & 2004 & 2006 & 2007 & 2004 & 2006 & 2007 & 2004 & 2006 & 2007 \\
\hline Unité de travail (éq. temps plein) & UTA par ferme & 3.2 & 3.9 & 4.0 & 0.7 & 0.6 & 0.6 & 1.6 & 1.8 & 1.8 & 2.3 & 2.9 & 2.8 \\
\hline Superficie agricole utilisée & Ha par ferme & 141.7 & 154.5 & 166.8 & 0.6 & 0.6 & 0.6 & 1.6 & 1.6 & 1.6 & 2.5 & 2.4 & 2.4 \\
\hline Production agricole brute ${ }^{1}$ & EUR par ferme & 516856 & 700770 & 745276 & 0.5 & 0.5 & 0.5 & 1.8 & 1.8 & 1.8 & 3.4 & 3.5 & 3.4 \\
\hline Soutien total & EUR par ferme & 165007 & 194702 & 175490 & 0.5 & 0.6 & 0.5 & 1.8 & 1.6 & 1.6 & 3.5 & 2.8 & 3.0 \\
\hline Tous paiements $^{2}$ & EUR par ferme & 53579 & 64897 & 65991 & 0.6 & 0.7 & 0.6 & 1.5 & 1.4 & 1.5 & 2.4 & 2.0 & 2.3 \\
\hline Paiements du 1er pilier & EUR par ferme & 398586.8 & 484105.0 & 491690.9 & 0.6 & 0.7 & 0.6 & 1.5 & 1.4 & 1.5 & 2.4 & 2.0 & 2.3 \\
\hline Paiements du 2ème pilier & EUR par ferme & n.a. & n.a. & n.a. & n.a. & n.a. & n.a. & n.a. & n.a & n.a. & n.a. & n.a. & n.a. \\
\hline Autres paiements & EUR par ferme & n.a. & n.a. & n.a. & n.a. & n.a. & n.a. & n.a. & n.a & n.a. & n.a. & n.a. & n.a. \\
\hline Soutien des prix du marché ${ }^{3}$ & EUR par ferme & 111428 & 129805 & 109499 & 0.5 & 0.5 & 0.4 & 1.9 & 1.7 & 1.6 & 4.2 & 3.4 & 3.7 \\
\hline Recettes brutes ${ }^{4}$ & EUR par ferme & 570435 & 765667 & 811267 & 0.5 & 0.5 & 0.5 & 1.8 & 1.8 & 1.8 & 3.3 & 3.3 & 3.3 \\
\hline Dépenses d'exploitation & EUR par ferme & 411645 & 583050 & 672907 & 0.5 & 0.5 & 0.5 & 1.9 & 1.9 & 1.8 & 3.6 & 3.6 & 3.6 \\
\hline Revenu net d'exploitation ${ }^{5}$ & EUR par ferme & 158789 & 182617 & 138359 & 0.6 & 0.6 & 0.7 & 1.5 & 1.7 & 1.4 & 2.6 & 2.6 & 2.1 \\
\hline Amortissements ${ }^{6}$ & EUR par ferme & 63788 & 79809 & 86131 & 0.5 & 0.5 & 0.6 & 1.8 & 1.8 & 1.8 & 3.4 & 3.3 & 3.2 \\
\hline Revenu agricole $^{7}$ & EUR par ferme & 95002 & 102809 & 52228 & 0.6 & 0.7 & 0.8 & 1.4 & 1.6 & 0.7 & 2.2 & 2.2 & 0.9 \\
\hline Revenu non agricole ${ }^{8}$ & EUR par ferme & 29405 & 30179 & 30849 & 1.0 & 0.8 & 0.9 & 1.1 & 1.2 & 1.1 & 1.0 & 1.6 & 1.2 \\
\hline Revenu des ménages agricoles ${ }^{9}$ & EUR par ferme & 124407 & 132988 & 83078 & 0.7 & 0.7 & 0.9 & 1.3 & 1.5 & 0.8 & 1.8 & 2.1 & 1.0 \\
\hline Total des actifs agricoles ${ }^{11}$ & EUR par ferme & n.a. & n.a. & n.a. & n.a. & n.a. & n.a. & n.a. & n.a & n.a. & n.a. & n.a. & n.a. \\
\hline Total des dettes agricoles ${ }^{12}$ & EUR par ferme & n.a. & n.a. & n.a. & n.a. & n.a. & n.a. & n.a. & n.a & n.a. & n.a. & n.a. & n.a. \\
\hline Capitaux propres agricoles ${ }^{13}$ & EUR par ferme & n.a. & n.a. & n.a. & n.a. & n.a. & n.a. & n.a. & n.a & n.a. & n.a. & n.a. & n.a. \\
\hline & & & & & & & Angleterr & & & & & & \\
\hline & Unité & Moyenn & $\begin{array}{l}\text { de l'ensembl } \\
\text { xploitations }\end{array}$ & & $\begin{array}{l}\text { Rapport ent } \\
\text { l'ensemble }\end{array}$ & $\begin{array}{l}\text { re le 1er } \\
\text { des expl }\end{array}$ & $\begin{array}{l}\text { uartile et } \\
\text { itations }\end{array}$ & $\begin{array}{l}\text { Rapport er } \\
\text { et l'ensemt }\end{array}$ & $\begin{array}{l}\text { tre le 4èn } \\
\text { le des ex }\end{array}$ & $\begin{array}{l}\text { quartile } \\
\text { pitations }\end{array}$ & Rapport en & $\begin{array}{l}\text { re le 4èm } \\
\text { quartiles }\end{array}$ & et le 1er \\
\hline & & 2004 & 2006 & 2007 & 2004 & 2006 & 2007 & 2004 & 2006 & 2007 & 2004 & 2006 & 2007 \\
\hline Unité de travail (éq. temps plein) & UTA par ferme & 2.7 & 2.8 & 2.8 & 0.4 & 0.4 & 0.4 & 2.1 & 2.3 & 2.2 & 5.2 & 5.5 & 5.5 \\
\hline Superficie agricole utilisée & Ha par ferme & 133.1 & 135.7 & 139.5 & 0.6 & 0.5 & 0.5 & 1.7 & 1.9 & 1.8 & 3.0 & 3.5 & 3.6 \\
\hline Production agricole brute ${ }^{1}$ & EUR par ferme & 206071 & 243882 & 284337 & 0.1 & 0.1 & 0.1 & 2.8 & 2.8 & 2.7 & 19.8 & 20.0 & 21.0 \\
\hline Soutien total & EUR par ferme & 105191 & 87015 & 91103 & 0.4 & 0.4 & 0.4 & 2.0 & 2.1 & 2.1 & 5.5 & 5.2 & 5.4 \\
\hline Tous paiements $^{2}$ & EUR par ferme & 59790 & 43476 & 44389 & 0.5 & 0.5 & 0.5 & 1.8 & 1.9 & 1.8 & 3.9 & 3.9 & 3.7 \\
\hline Paiements du 1er pilier & EUR par ferme & 54881 & 36001 & 35352 & 0.4 & 0.4 & 0.4 & 1.8 & 1.9 & 1.9 & 4.5 & 4.6 & 4.4 \\
\hline Paiements du 2ème pilier & EUR par ferme & 4336 & 7270 & 8735 & 0.9 & 0.8 & 0.7 & 1.1 & 1.6 & 1.6 & 1.2 & 2.0 & 2.2 \\
\hline Autres paiements & EUR par ferme & 573 & 206 & 302 & 0.8 & 0.4 & 1.3 & 1.4 & 2.0 & 1.3 & 1.8 & 5.0 & 1.0 \\
\hline Soutien des prix du marché ${ }^{3}$ & EUR par ferme & 45401 & 43538 & 46715 & 0.3 & 0.3 & 0.3 & 2.3 & 2.2 & 2.3 & 9.3 & 7.3 & 8.1 \\
\hline Recettes brutes ${ }^{4}$ & EUR par ferme & 265862 & 287358 & 328725 & 0.2 & 0.2 & 0.2 & 2.6 & 2.6 & 2.6 & 12.1 & 13.8 & 14.6 \\
\hline Dépenses d'exploitation & EUR par ferme & 199819 & 211132 & 230607 & 0.2 & 0.2 & 0.2 & 2.6 & 2.7 & 2.7 & 12.6 & 13.6 & 14.1 \\
\hline Revenu net d'exploitation ${ }^{5}$ & EUR par ferme & 66043 & 76226 & 98118 & 0.2 & 0.2 & 0.1 & 2.4 & 2.5 & 2.4 & 10.8 & 14.6 & 16.3 \\
\hline Amortissements ${ }^{6}$ & EUR par ferme & 24341 & 25852 & 27741 & 0.3 & 0.3 & 0.3 & 2.2 & 2.4 & 2.4 & 7.5 & 8.7 & 8.4 \\
\hline Revenu agricole $^{7}$ & EUR par ferme & 41702 & 50375 & 70377 & 0.2 & 0.1 & 0.1 & 2.4 & 2.5 & 2.5 & 14.1 & 22.2 & 25.3 \\
\hline Revenu non agricole ${ }^{8}$ & EUR par ferme & n.a. & n.a. & n.a. & n.a. & n.a. & n.a. & n.a. & n.a. & n.a. & n.a. & n.a. & n.a. \\
\hline Revenu des ménages agricoles ${ }^{9}$ & EUR par ferme & n.a. & n.a. & n.a. & n.a. & n.a. & n.a. & n.a. & n.a. & n.a. & n.a. & n.a. & n.a. \\
\hline Total des actifs agricoles ${ }^{11}$ & EUR par ferme & 1069338 & 1192958 & 1411032 & 0.5 & 0.5 & 0.5 & 1.8 & 1.9 & 1.9 & 3.7 & 4.0 & 3.7 \\
\hline Total des dettes agricoles ${ }^{12}$ & EUR par ferme & 139728 & 154429 & 172197 & 0.3 & 0.2 & 0.2 & 2.4 & 2.4 & 2.5 & 9.3 & 10.2 & 11.2 \\
\hline Capitaux propres agricoles ${ }^{13}$ & EUR par ferme & 929610 & 1038529 & 1238834 & 0.5 & 0.5 & 0.6 & 1.7 & 1.8 & 1.8 & 3.3 & 3.6 & 3.2 \\
\hline
\end{tabular}


DISTRIBUTION DU SOUTIEN ET DES REVENUS DANS L'AGRICULTURE - 73

\begin{tabular}{|c|c|c|c|c|c|c|c|c|c|c|c|c|c|}
\hline & \multicolumn{13}{|c|}{ Espagne } \\
\hline & \multirow[t]{2}{*}{ Unité } & \multicolumn{3}{|c|}{$\begin{array}{c}\text { Moyenne de l'ensemble des } \\
\text { exploitations }\end{array}$} & \multicolumn{3}{|c|}{$\begin{array}{l}\text { Rapport entre le 1er quartile et } \\
\text { l'ensemble des exploitations }\end{array}$} & \multicolumn{3}{|c|}{$\begin{array}{l}\text { Rapport entre le 4ème quartile } \\
\text { et l'ensemble des exploitations }\end{array}$} & \multicolumn{3}{|c|}{$\begin{array}{l}\text { Rapport entre le 4ème et le 1er } \\
\text { quartiles }\end{array}$} \\
\hline & & 2004 & 2006 & 2007 & 2004 & 2006 & 2007 & 2004 & 2006 & 2007 & 2004 & 2006 & 2007 \\
\hline Unité de travail (éq. temps plein) & UTA par ferme & 1.1 & 1.0 & 1.0 & 0.9 & 0.7 & 0.6 & 1.3 & 1.3 & 1.6 & 1.4 & 1.9 & 2.7 \\
\hline Superficie agricole utilisée & Ha par ferme & 25.6 & 25.7 & 25.8 & 0.3 & 0.2 & 0.3 & 2.4 & 2.4 & 2.5 & 9.2 & 9.7 & 8.6 \\
\hline Production agricole brute ${ }^{1}$ & EUR par ferme & 36184 & 32332 & 39275 & 0.4 & 0.3 & 0.2 & 2.2 & 2.3 & 2.5 & 5.8 & 7.1 & 11.9 \\
\hline Soutien total & EUR par ferme & n.a. & n.a. & n.a. & n.a. & n.a. & n.a. & n.a. & n.a. & n.a. & n.a. & n.a. & n.a. \\
\hline Tous paiements ${ }^{2}$ & EUR par ferme & 6253 & 6517 & 5208 & 0.5 & 0.5 & 0.4 & 2.2 & 2.2 & 2.4 & 4.4 & 4.1 & 6.7 \\
\hline Paiements du 1er pilier & EUR par ferme & n.a. & n.a. & n.a. & n.a. & n.a. & n.a. & n.a. & n.a. & n.a. & n.a. & n.a. & n.a. \\
\hline Paiements du 2ème pilier & EUR par ferme & n.a. & n.a. & n.a. & n.a. & n.a. & n.a. & n.a. & n.a. & n.a. & n.a. & n.a. & n.a. \\
\hline Autres paiements & EUR par ferme & n.a. & n.a. & n.a. & n.a. & n.a. & n.a. & n.a. & n.a. & n.a. & n.a. & n.a. & n.a. \\
\hline Soutien des prix du marché ${ }^{3}$ & EUR par ferme & n.a. & n.a. & n.a. & n.a. & n.a. & n.a. & n.a. & n.a. & n.a. & n.a. & n.a. & n.a. \\
\hline Recettes brutes ${ }^{4}$ & EUR par ferme & 42437 & 38849 & 44483 & 0.4 & 0.4 & 0.2 & 2.2 & 2.3 & 2.5 & 5.6 & 6.4 & 10.9 \\
\hline Dépenses d'exploitation & EUR par ferme & 19430 & 18090 & 20348 & 0.3 & 0.3 & 0.2 & 2.3 & 2.4 & 2.6 & 6.9 & 8.5 & 11.5 \\
\hline Revenu net d'exploitation ${ }^{5}$ & EUR par ferme & 23007 & 20759 & 24135 & 0.4 & 0.4 & 0.2 & 2.1 & 2.1 & 2.4 & 4.7 & 5.1 & 10.4 \\
\hline Amortissements ${ }^{6}$ & EUR par ferme & 1904 & 2267 & 2264 & 0.5 & 0.8 & 0.3 & 2.0 & 1.8 & 2.2 & 3.7 & 2.2 & 6.3 \\
\hline Revenu agricole $^{7}$ & EUR par ferme & 21103 & 18492 & 21871 & 0.4 & 0.4 & 0.2 & 2.1 & 2.2 & 2.4 & 4.8 & 5.9 & 11.1 \\
\hline Revenu non agricole ${ }^{8}$ & EUR par ferme & n.a. & n.a. & n.a. & n.a. & n.a. & n.a. & n.a. & n.a. & n.a. & n.a. & n.a. & n.a. \\
\hline Revenu des ménages agricoles ${ }^{9}$ & EUR par ferme & n.a. & n.a. & n.a. & n.a. & n.a. & n.a. & n.a. & n.a. & n.a. & n.a. & n.a. & n.a. \\
\hline Total des actifs agricoles ${ }^{11}$ & EUR par ferme & 180450 & 216333 & 255531 & 0.6 & 0.5 & 0.6 & 1.8 & 1.8 & 1.8 & 3.1 & 3.3 & 3.1 \\
\hline Total des dettes agricoles ${ }^{12}$ & EUR par ferme & 5108 & 4139 & 4164 & 0.5 & 0.3 & 0.2 & 2.3 & 2.3 & 2.7 & 4.4 & 7.3 & 11.7 \\
\hline \multirow[t]{4}{*}{ Capitaux propres agricoles ${ }^{13}$} & EUR par ferme & 175342 & 212194 & 251367 & 0.6 & 0.5 & 0.6 & 1.8 & 1.8 & 1.8 & 3.0 & 3.3 & 3.0 \\
\hline & \multicolumn{13}{|c|}{ Estonia } \\
\hline & Unit & \multicolumn{3}{|c|}{$\begin{array}{l}\text { Moyenne de l'ensemble des } \\
\text { exploitations }\end{array}$} & $\begin{array}{l}\text { Rapport } \\
\text { l'ensem }\end{array}$ & $\begin{array}{l}\text { tre le 1er } \\
\text { e des exp }\end{array}$ & $\begin{array}{l}\text { Jartile et } \\
\text { tations }\end{array}$ & $\begin{array}{l}\text { Rapport } \\
\text { et l'enser }\end{array}$ & $\begin{array}{l}\text { tre le 4èr } \\
\text { le des ex }\end{array}$ & $\begin{array}{l}\text { quartile } \\
\text { bitations }\end{array}$ & Rapport & $\begin{array}{l}\text { re le 4èn } \\
\text { quartiles }\end{array}$ & et le 1er \\
\hline & & 2004 & 2006 & 2007 & 2004 & 2006 & 2007 & 2004 & 2006 & 2007 & 2004 & 2006 & 2007 \\
\hline Unité de travail (éq. temps plein) & UTA par ferme & 3.4 & 3.0 & 2.8 & 0.4 & 0.5 & 0.5 & 2.5 & 2.3 & 2.3 & 5.7 & 5.0 & 4.5 \\
\hline Superficie agricole utilisée & Ha par ferme & 119.1 & 118.2 & 123.9 & 0.4 & 0.4 & 0.4 & 2.7 & 2.7 & 2.7 & 7.5 & 7.5 & 7.5 \\
\hline Production agricole brute ${ }^{1}$ & EUR par ferme & 63705 & 70185 & 82802 & 0.1 & 0.1 & 0.1 & 3.4 & 3.4 & 3.4 & 34.2 & 35.7 & 38.1 \\
\hline Soutien total & EUR par ferme & 26864 & 24181 & 23241 & 0.2 & 0.3 & 0.3 & 3.2 & 2.9 & 2.7 & 15.9 & 9.8 & 8.1 \\
\hline Tous paiements $^{2}$ & EUR par ferme & 16060 & 17280 & 19810 & 0.3 & 0.4 & 0.4 & 3.0 & 2.7 & 2.8 & 10.7 & 7.4 & 7.7 \\
\hline Paiements du 1er pilier & EUR par ferme & 6589 & 9372 & 11879 & 0.3 & 0.3 & 0.2 & 2.9 & 2.9 & 3.1 & 10.3 & 11.5 & 13.9 \\
\hline Paiements du 2ème pilier & EUR par ferme & 9161 & 7366 & 7913 & 0.3 & 0.5 & 0.6 & 3.1 & 2.5 & 2.3 & 11.5 & 5.0 & 4.1 \\
\hline Autres paiements & EUR par ferme & 310 & 542 & 18 & 0.5 & 0.5 & 0.0 & 1.6 & 2.3 & 4.0 & 3.3 & 4.7 & n.a. \\
\hline Soutien des prix du marché ${ }^{3}$ & EUR par ferme & 10804 & 6901 & 3431 & 0.1 & 0.1 & 0.2 & 3.4 & 3.2 & 2.7 & 42.9 & 32.3 & 11.9 \\
\hline Recettes brutes ${ }^{4}$ & EUR par ferme & 79765 & 87465 & 102612 & 0.1 & 0.1 & 0.1 & 3.3 & 3.3 & 3.3 & 24.5 & 21.9 & 23.3 \\
\hline Dépenses d'exploitation & EUR par ferme & 54319 & 65404 & 68735 & 0.1 & 0.1 & 0.1 & 3.4 & 3.4 & 3.4 & 28.8 & 35.1 & 27.8 \\
\hline Revenu net d'exploitation ${ }^{5}$ & EUR par ferme & 25446 & 22061 & 33877 & 0.2 & 0.3 & 0.2 & 3.2 & 2.9 & 3.1 & 18.2 & 9.4 & 17.3 \\
\hline Amortissements ${ }^{6}$ & EUR par ferme & 6749 & 7252 & 9498 & 0.2 & 0.1 & 0.2 & 3.2 & 3.3 & 3.3 & 20.7 & 25.2 & 18.1 \\
\hline Revenu agricole ${ }^{7}$ & EUR par ferme & 18698 & 14809 & 24379 & 0.2 & 0.4 & 0.2 & 3.1 & 2.6 & 3.1 & 17.4 & 6.8 & 17.0 \\
\hline Revenu non agricole ${ }^{8}$ & EUR par ferme & n.a. & n.a. & n.a. & n.a. & n.a. & n.a. & n.a. & n.a. & n.a. & n.a. & n.a. & n.a. \\
\hline Revenu des ménages agricoles ${ }^{9}$ & EUR par ferme & n.a. & n.a. & n.a. & n.a. & n.a. & n.a. & n.a. & n.a. & n.a. & n.a. & n.a. & n.a. \\
\hline Total des actifs agricoles ${ }^{11}$ & EUR par ferme & 143827 & 173618 & 196331 & 0.3 & 0.3 & 0.3 & 3.0 & 2.8 & 2.8 & 11.1 & 9.9 & 8.4 \\
\hline Total des dettes agricoles ${ }^{12}$ & EUR par ferme & 31416 & 48428 & 53127 & 0.1 & 0.1 & 0.1 & 3.6 & 3.5 & 3.5 & 63.3 & 60.9 & 27.5 \\
\hline Capitaux propres agricoles ${ }^{13}$ & EUR par ferme & 112411 & 125190 & 143204 & 0.3 & 0.4 & 0.4 & 2.9 & 2.5 & 2.6 & 8.7 & 6.8 & 6.3 \\
\hline & & & & & & & landre $b$ & & & & & & \\
\hline & Unité & Moyenne & $\begin{array}{l}\text { de l'ensemb } \\
\text { ploitations }\end{array}$ & & $\begin{array}{l}\text { Rapport } \\
\text { l'ensem }\end{array}$ & $\begin{array}{l}t \text { tre le } 1 \mathrm{er} \\
\text { e des exp }\end{array}$ & $\begin{array}{l}\text { Jartile et } \\
\text { tations }\end{array}$ & $\begin{array}{l}\text { Rapport } \\
\text { et l'ense }\end{array}$ & $\begin{array}{l}\text { tre le 4è } \\
\text { le des ex }\end{array}$ & $\begin{array}{l}\text { quartile } \\
\text { oitations }\end{array}$ & Rapport & $\begin{array}{l}\text { tre le 4è } \\
\text { quartiles }\end{array}$ & et le 1er \\
\hline & & 2004 & 2006 & 2007 & 2004 & 2006 & 2007 & 2004 & 2006 & 2007 & 2004 & 2006 & 2007 \\
\hline Unité de travail (éq. temps plein) & UTA par ferme & 2.0 & 2.0 & 2.1 & 0.7 & 0.6 & 0.6 & 1.5 & 1.6 & 1.7 & 2.2 & 2.7 & 2.7 \\
\hline Superficie agricole utilisée & Ha par ferme & 28.8 & 30.1 & 32.0 & 0.7 & 0.7 & 0.7 & 1.1 & 1.2 & 1.3 & 1.6 & 1.6 & 1.8 \\
\hline Production agricole brute ${ }^{1}$ & EUR par ferme & 196045 & 211536 & 235374 & 0.2 & 0.2 & 0.2 & 2.3 & 2.3 & 2.3 & 9.2 & 10.2 & 10.1 \\
\hline Soutien total & EUR par ferme & 45055 & 37138 & 36153 & 0.4 & 0.5 & 0.5 & 1.6 & 1.5 & 1.6 & 3.5 & 2.9 & 3.2 \\
\hline Tous paiements $^{2}$ & EUR par ferme & 10975 & 14961 & 17603 & 0.7 & 0.7 & 0.6 & 1.1 & 1.2 & 1.3 & 1.4 & 1.8 & 2.2 \\
\hline Paiements du 1er pilier & EUR par ferme & 8616 & 11803 & 12969 & 0.8 & 0.7 & 0.6 & 0.9 & 1.0 & 1.2 & 1.1 & 1.5 & 1.8 \\
\hline Paiements du 2ème pilier & EUR par ferme & 1945 & 2600 & 3937 & 0.4 & 0.4 & 0.4 & 1.5 & 1.8 & 1.8 & 3.4 & 4.2 & 4.8 \\
\hline Autres paiements & EUR par ferme & 414 & 558 & 697 & 0.2 & 0.7 & 0.7 & 1.9 & 1.5 & 1.4 & 9.9 & 2.1 & 1.9 \\
\hline Soutien des prix du marché ${ }^{3}$ & EUR par ferme & 34080 & 22177 & 18550 & 0.3 & 0.4 & 0.4 & 1.7 & 1.6 & 1.9 & 5.0 & 4.0 & 4.4 \\
\hline Recettes brutes ${ }^{4}$ & EUR par ferme & 207020 & 226497 & 252977 & 0.3 & 0.3 & 0.3 & 2.2 & 2.3 & 2.2 & 8.1 & 8.8 & 8.8 \\
\hline Dépenses d'exploitation & EUR par ferme & 136595 & 141410 & 161518 & 0.3 & 0.2 & 0.2 & 2.3 & 2.4 & 2.4 & 9.0 & 9.7 & 10.0 \\
\hline Revenu net d'exploitation ${ }^{5}$ & EUR par ferme & 70425 & 85087 & 91459 & 0.3 & 0.3 & 0.3 & 2.0 & 2.0 & 2.0 & 6.5 & 7.4 & 7.0 \\
\hline Amortissements ${ }^{6}$ & EUR par ferme & 23207 & 23186 & 27161 & 0.3 & 0.4 & 0.3 & 1.9 & 2.0 & 2.0 & 5.7 & 5.1 & 6.0 \\
\hline Revenu agricole ${ }^{7}$ & EUR par ferme & 47218 & 61901 & 64298 & 0.3 & 0.2 & 0.3 & 2.0 & 2.0 & 1.9 & 7.0 & 8.8 & 7.6 \\
\hline Revenu non agricole ${ }^{8}$ & EUR par ferme & n.a. & n.a. & n.a. & n.a. & n.a. & n.a. & n.a. & n.a. & n.a. & n.a. & n.a. & n.a. \\
\hline Revenu des ménages agricoles ${ }^{9}$ & EUR par ferme & n.a. & n.a. & n.a. & n.a. & n.a. & n.a. & n.a. & n.a. & n.a. & n.a. & n.a. & n.a. \\
\hline Total des actifs agricoles ${ }^{11}$ & EUR par ferme & 413931 & 470743 & 527932 & 0.5 & 0.5 & 0.4 & 1.7 & 1.7 & 1.8 & 3.3 & 3.6 & 4.1 \\
\hline Total des dettes agricoles ${ }^{12}$ & EUR par ferme & 126394 & 129889 & 146413 & 0.4 & 0.3 & 0.3 & 2.0 & 2.1 & 2.2 & 5.4 & 6.8 & 8.1 \\
\hline Capitaux propres agricoles ${ }^{13}$ & EUR par ferme & 287537 & 340854 & 381519 & 0.6 & 0.5 & 0.5 & 1.5 & 1.6 & 1.6 & 2.7 & 2.9 & 3.3 \\
\hline
\end{tabular}




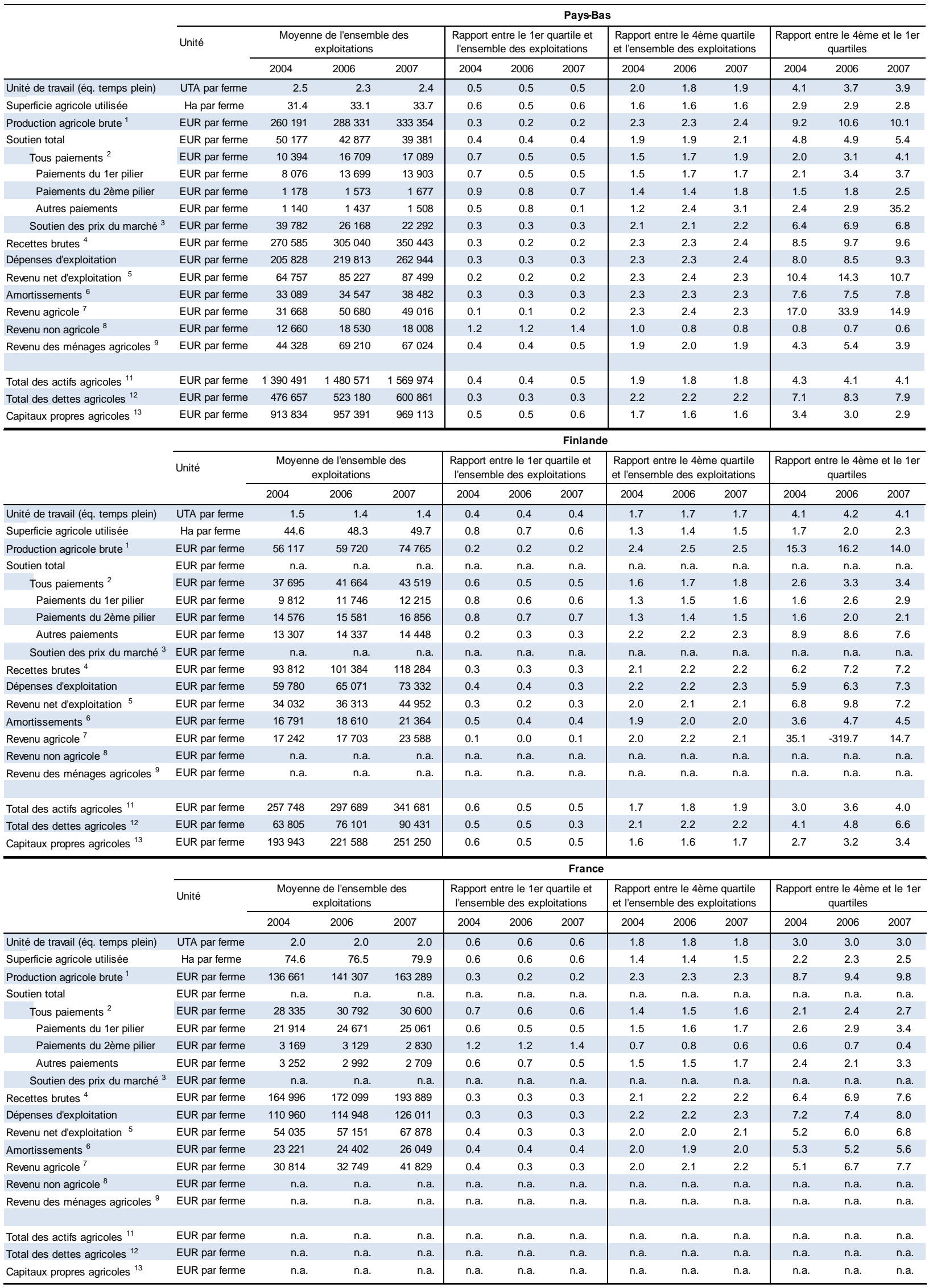




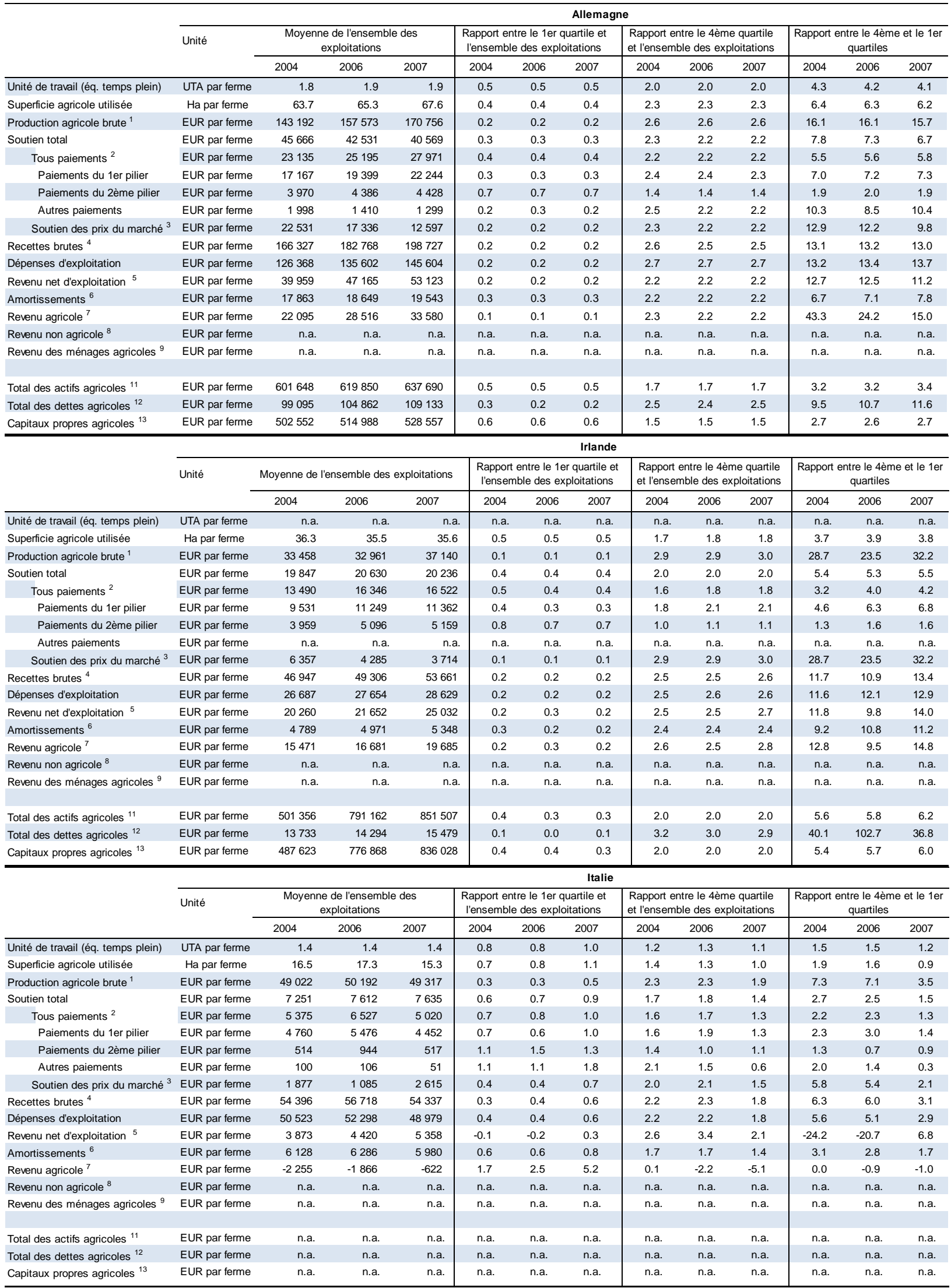

Notes identiques à celles du tableau $\mathrm{C} 1$.

Source : tableaux transmis par les participants. 
Tableau annexe C4. Part du soutien dans les recettes des exploitations et dans le revenu agricole, par taille d'exploitation, certains pays de l'OCDE, 2004, 2006 et 2007

\begin{tabular}{|c|c|c|c|c|c|c|c|c|c|}
\hline \multirow{3}{*}{$\begin{array}{l}\text { Pays } \\
\text { Quartile } \\
\text { Année }\end{array}$} & \multicolumn{9}{|c|}{ Canada } \\
\hline & \multicolumn{3}{|c|}{ Toutes exploitations } & \multicolumn{3}{|c|}{ 1er quartile } & \multicolumn{3}{|c|}{ 4ème quartile } \\
\hline & 2004 & 2006 & 2007 & 2004 & 2006 & 2007 & 2004 & 2006 & 2007 \\
\hline Part du soutien dans les recettes brutes ${ }^{1}$ & 15 & 17 & 13 & 26 & 28 & 20 & 12 & 14 & 11 \\
\hline Tous paiements $^{2}$ & 9 & 9 & 7 & 24 & 26 & 18 & 6 & 6 & 6 \\
\hline Paiements du 1er pilier & n.a. & n.a. & n.a. & n.a. & n.a. & n.a. & n.a. & n.a. & n.a. \\
\hline Paiements du 2ème pilier & n.a. & n.a. & n.a. & n.a. & n.a. & n.a. & n.a. & n.a. & n.a. \\
\hline Autres paiements & 9 & 9 & 7 & 24 & 26 & 18 & 6 & 6 & 6 \\
\hline Soutien des prix du marché ${ }^{3}$ & 7 & 8 & 6 & 2 & 2 & 2 & 6 & 8 & 6 \\
\hline Part du soutien dans le revenu agricole & 280 & 384 & 211 & 654 & 606 & 382 & 226 & 371 & 199 \\
\hline Pays & \multicolumn{9}{|c|}{ États-Unis } \\
\hline Quartile & \multicolumn{3}{|c|}{ Toutes exploitations } & \multicolumn{3}{|c|}{ 1er quartile } & \multicolumn{3}{|c|}{ 4ème quartile } \\
\hline Année & 2004 & 2006 & 2007 & 2004 & 2006 & 2007 & 2004 & 2006 & 2007 \\
\hline Part du soutien dans les recettes brutes ${ }^{1}$ & 9 & 6 & 7 & 9 & 8 & 7 & 8 & 5 & 7 \\
\hline Tous paiements $^{2}$ & 4 & 4 & 3 & 7 & 7 & 5 & 3 & 3 & 2 \\
\hline Paiements du 1er pilier & 0 & 0 & 0 & 0 & 0 & 0 & 0 & 0 & 0 \\
\hline Paiements du 2ème pilier & 0 & 0 & 0 & 0 & 0 & 0 & 0 & 0 & 0 \\
\hline Autres paiements & 0 & 0 & 0 & 0 & 0 & 0 & 0 & 0 & 0 \\
\hline Soutien des prix du marché ${ }^{3}$ & 4 & 2 & 4 & 2 & 1 & 2 & 5 & 2 & 4 \\
\hline Part du soutien dans le revenu agricole & 39 & 37 & 33 & 63 & 68 & 70 & 32 & 29 & 30 \\
\hline Pays & \multicolumn{9}{|c|}{ UE27 } \\
\hline Quartile & \multicolumn{3}{|c|}{ Toutes exploitations } & \multicolumn{3}{|c|}{ 1er quartile } & \multicolumn{3}{|c|}{ 4ème quartile } \\
\hline Année & 2004 & 2006 & 2007 & 2004 & 2006 & 2007 & 2004 & 2006 & 2007 \\
\hline Part du soutien dans les recettes brutes ${ }^{1}$ & 27 & 24 & 20 & 36 & 41 & 31 & 27 & 22 & 18 \\
\hline Tous paiements $^{2}$ & 15 & 16 & 14 & 30 & 37 & 27 & 12 & 13 & 12 \\
\hline Paiements du 1er pilier & 12 & 13 & 12 & 24 & 27 & 20 & 11 & 11 & 11 \\
\hline Paiements du 2ème pilier & 2 & 3 & 2 & 5 & 10 & 7 & 2 & 2 & 2 \\
\hline Autres paiements & 0 & 0 & 0 & 0 & 0 & 0 & 0 & 0 & 0 \\
\hline Soutien des prix du marché ${ }^{3}$ & 13 & 8 & 6 & 7 & 4 & 5 & 14 & 9 & 6 \\
\hline Part du soutien dans le revenu agricole & 107 & 93 & 71 & 136 & 133 & 114 & 119 & 97 & 72 \\
\hline Pays & \multicolumn{9}{|c|}{ UE15 } \\
\hline Quartile & \multicolumn{3}{|c|}{ Toutes exploitations } & \multicolumn{3}{|c|}{ 1er quartile } & \multicolumn{3}{|c|}{ 4ème quartile } \\
\hline Année & 2004 & 2006 & 2007 & 2004 & 2006 & 2007 & 2004 & 2006 & 2007 \\
\hline Part du soutien dans les recettes brutes ${ }^{1}$ & 28 & 24 & 20 & 36 & 40 & 35 & 26 & 22 & 17 \\
\hline Tous paiements $^{2}$ & 15 & 16 & 14 & 32 & 37 & 31 & 12 & 13 & 11 \\
\hline Paiements du 1er pilier & 13 & 13 & 12 & 27 & 30 & 25 & 10 & 11 & 10 \\
\hline Paiements du 2ème pilier & 2 & 3 & 2 & 6 & 7 & 6 & 2 & 2 & 1 \\
\hline Autres paiements & 0 & 0 & 0 & 0 & 0 & 0 & 0 & 0 & 0 \\
\hline Soutien des prix du marché ${ }^{3}$ & 13 & 8 & 6 & 4 & 3 & 4 & 14 & 9 & 6 \\
\hline Part du soutien dans le revenu agricole & 105 & 90 & 70 & 102 & 112 & 84 & 116 & 93 & 68 \\
\hline
\end{tabular}




\begin{tabular}{|c|c|c|c|c|c|c|c|c|c|}
\hline \multirow{3}{*}{$\begin{array}{l}\text { Pays } \\
\text { Quartile } \\
\text { Année }\end{array}$} & \multicolumn{9}{|c|}{ Danemark } \\
\hline & \multicolumn{3}{|c|}{ Toutes exploitations } & \multicolumn{3}{|c|}{ 1er quartile } & \multicolumn{3}{|c|}{ 4ème quartile } \\
\hline & 2004 & 2006 & 2007 & 2004 & 2006 & 2007 & 2004 & 2006 & 2007 \\
\hline Part du soutien dans les recettes brutes ${ }^{1}$ & 29 & 25 & 22 & 27 & 27 & 21 & 29 & 23 & 19 \\
\hline Tous paiements $^{2}$ & 9 & 8 & 8 & 11 & 11 & 10 & 8 & 7 & 7 \\
\hline Paiements du 1er pilier & 9 & 8 & 8 & 11 & 11 & 10 & 8 & 7 & 7 \\
\hline Paiements du 2ème pilier & 0 & 0 & 0 & 0 & 0 & 0 & 0 & 0 & 0 \\
\hline Autres paiements & 0 & 0 & 0 & 0 & 0 & 0 & 0 & 0 & 0 \\
\hline Soutien des prix du marché ${ }^{3}$ & 20 & 17 & 13 & 17 & 16 & 11 & 21 & 16 & 13 \\
\hline Part du soutien dans le revenu agricole & 174 & 189 & 336 & 144 & 155 & 217 & 225 & 191 & 760 \\
\hline Pays & \multicolumn{9}{|c|}{ Angleterre } \\
\hline Quartile & \multicolumn{3}{|c|}{ Toutes exploitations } & \multicolumn{3}{|c|}{ 1er quartile } & \multicolumn{3}{|c|}{ 4ème quartile } \\
\hline Année & 2004 & 2006 & 2007 & 2004 & 2006 & 2007 & 2004 & 2006 & 2007 \\
\hline Part du soutien dans les recettes brutes ${ }^{1}$ & 40 & 30 & 28 & 69 & 63 & 60 & 31 & 24 & 22 \\
\hline Tous paiements $^{2}$ & 22 & 15 & 14 & 48 & 38 & 37 & 16 & 11 & 9 \\
\hline Paiements du 1er pilier & 21 & 13 & 11 & 40 & 28 & 26 & 15 & 9 & 8 \\
\hline Paiements du 2ème pilier & 2 & 3 & 3 & 7 & 10 & 11 & 1 & 1 & 2 \\
\hline Autres paiements & 0 & 0 & 0 & 1 & 0 & 1 & 0 & 0 & 0 \\
\hline Soutien des prix du marché ${ }^{3}$ & 17 & 15 & 14 & 20 & 25 & 23 & 16 & 13 & 13 \\
\hline Part du soutien dans le revenu agricole & 252 & 173 & 129 & 538 & 604 & 509 & 211 & 142 & 109 \\
\hline Pays & \multicolumn{9}{|c|}{ Espagne } \\
\hline Quartile & \multicolumn{3}{|c|}{ Toutes exploitations } & \multicolumn{3}{|c|}{ 1er quartile } & \multicolumn{3}{|c|}{ 4ème quartile } \\
\hline Année & 2004 & 2006 & 2007 & 2004 & 2006 & 2007 & 2004 & 2006 & 2007 \\
\hline Part du soutien dans les recettes brutes ${ }^{1}$ & n.a. & n.a. & n.a. & n.a. & n.a. & n.a. & n.a. & n.a. & n.a. \\
\hline Tous paiements ${ }^{2}$ & 15 & 17 & 12 & 18 & 25 & 18 & 15 & 16 & 11 \\
\hline Paiements du 1er pilier & n.a. & n.a. & n.a. & n.a. & n.a. & n.a. & n.a. & n.a. & n.a. \\
\hline Paiements du 2ème pilier & n.a. & n.a. & n.a. & n.a. & n.a. & n.a. & n.a. & n.a. & n.a. \\
\hline Autres paiements & n.a. & n.a. & n.a. & n.a. & n.a. & n.a. & n.a. & n.a. & n.a. \\
\hline Soutien des prix du marché ${ }^{3}$ & n.a. & n.a. & n.a. & n.a. & n.a. & n.a. & n.a. & n.a. & n.a. \\
\hline Part du soutien dans le revenu agricole & n.a. & n.a. & n.a. & n.a. & n.a. & n.a. & n.a. & n.a. & n.a. \\
\hline Pays & \multicolumn{9}{|c|}{ Estonie } \\
\hline Quartile & \multicolumn{3}{|c|}{ Toutes exploitations } & \multicolumn{3}{|c|}{ 1er quartile } & \multicolumn{3}{|c|}{ 4ème quartile } \\
\hline Année & 2004 & 2006 & 2007 & 2004 & 2006 & 2007 & 2004 & 2006 & 2007 \\
\hline Part du soutien dans les recettes brutes ${ }^{1}$ & 34 & 28 & 23 & 49 & 54 & 54 & 32 & 24 & 19 \\
\hline Tous paiements $^{2}$ & 20 & 20 & 19 & 42 & 49 & 49 & 18 & 16 & 16 \\
\hline Paiements du 1er pilier & 8 & 11 & 12 & 17 & 18 & 18 & 7 & 10 & 11 \\
\hline Paiements du 2ème pilier & 11 & 8 & 8 & 23 & 28 & 31 & 11 & 6 & 5 \\
\hline Autres paiements & 0 & 1 & 0 & 1 & 2 & 0 & 0 & 0 & 0 \\
\hline Soutien des prix du marché ${ }^{3}$ & 14 & 8 & 3 & 8 & 5 & 5 & 14 & 8 & 3 \\
\hline Part du soutien dans le revenu agricole & 144 & 163 & 95 & 159 & 123 & 178 & 145 & 177 & 85 \\
\hline
\end{tabular}




\begin{tabular}{|c|c|c|c|c|c|c|c|c|c|}
\hline \multirow{3}{*}{$\begin{array}{l}\text { Pays } \\
\text { Quartile } \\
\text { Année }\end{array}$} & \multicolumn{9}{|c|}{ Flandre belge } \\
\hline & \multicolumn{3}{|c|}{ Toutes exploitations } & \multicolumn{3}{|c|}{ 1er quartile } & \multicolumn{3}{|c|}{ 4ème quartile } \\
\hline & 2004 & 2006 & 2007 & 2004 & 2006 & 2007 & 2004 & 2006 & 2007 \\
\hline Part du soutien dans les recettes brutes ${ }^{1}$ & 22 & 16 & 14 & 35 & 32 & 29 & 15 & 11 & 10 \\
\hline Tous paiements $^{2}$ & 5 & 7 & 7 & 15 & 17 & 16 & 3 & 3 & 4 \\
\hline Paiements du 1er pilier & 4 & 5 & 5 & 13 & 14 & 13 & 2 & 2 & 3 \\
\hline Paiements du 2ème pilier & 1 & 1 & 2 & 2 & 2 & 2 & 1 & 1 & 1 \\
\hline Autres paiements & 0 & 0 & 0 & 0 & 1 & 1 & 0 & 0 & 0 \\
\hline Soutien des prix du marché ${ }^{3}$ & 16 & 10 & 7 & 21 & 16 & 13 & 13 & 7 & 6 \\
\hline Part du soutien dans le revenu agricole & 95 & 60 & 56 & 146 & 131 & 115 & 73 & 43 & 48 \\
\hline Pays & \multicolumn{9}{|c|}{ Pays-Bas } \\
\hline Quartile & \multicolumn{3}{|c|}{ Toutes exploitations } & \multicolumn{3}{|c|}{ 1er quartile } & \multicolumn{3}{|c|}{ 4ème quartile } \\
\hline Année & 2004 & 2006 & 2007 & 2004 & 2006 & 2007 & 2004 & 2006 & 2007 \\
\hline Part du soutien dans les recettes brutes ${ }^{1}$ & 19 & 14 & 11 & 28 & 23 & 17 & 16 & 12 & 10 \\
\hline Tous paiements $^{2}$ & 4 & 5 & 5 & 10 & 12 & 9 & 2 & 4 & 4 \\
\hline Paiements du 1er pilier & 3 & 4 & 4 & 8 & 9 & 7 & 2 & 3 & 3 \\
\hline Paiements du 2ème pilier & 0 & 1 & 0 & 1 & 2 & 1 & 0 & 0 & 0 \\
\hline Autres paiements & 0 & 0 & 0 & 1 & 2 & 0 & 0 & 0 & 1 \\
\hline Soutien des prix du marché ${ }^{3}$ & 15 & 9 & 6 & 18 & 11 & 8 & 13 & 8 & 6 \\
\hline Part du soutien dans le revenu agricole & 158 & 85 & 80 & 485 & 466 & 194 & 136 & 67 & 70 \\
\hline Pays & \multicolumn{9}{|c|}{ Finlande } \\
\hline Quartile & \multicolumn{3}{|c|}{ Toutes exploitations } & \multicolumn{3}{|c|}{ 1er quartile } & \multicolumn{3}{|c|}{ 4ème quartile } \\
\hline Année & 2004 & 2006 & 2007 & 2004 & 2006 & 2007 & 2004 & 2006 & 2007 \\
\hline Part du soutien dans les recettes brutes ${ }^{1}$ & n.a. & n.a. & n.a. & n.a. & n.a. & n.a. & n.a. & n.a. & n.a. \\
\hline Tous paiements $^{2}$ & 40 & 41 & 37 & 72 & 70 & 64 & 31 & 32 & 30 \\
\hline Paiements du 1er pilier & 10 & 12 & 10 & 24 & 23 & 19 & 6 & 8 & 8 \\
\hline Paiements du 2ème pilier & 16 & 15 & 14 & 38 & 35 & 33 & 10 & 10 & 9 \\
\hline Autres paiements & 14 & 14 & 12 & 10 & 12 & 12 & 15 & 14 & 13 \\
\hline Soutien des prix du marché ${ }^{3}$ & n.a. & n.a. & n.a. & n.a. & n.a. & n.a. & n.a. & n.a. & n.a. \\
\hline Part du soutien dans le revenu agricole & n.a. & n.a. & n.a. & n.a. & n.a. & n.a. & n.a. & n.a. & n.a. \\
\hline Pays & \multicolumn{9}{|c|}{ France } \\
\hline Quartile & \multicolumn{3}{|c|}{ Toutes exploitations } & \multicolumn{3}{|c|}{ 1er quartile } & \multicolumn{3}{|c|}{ 4ème quartile } \\
\hline Année & 2004 & 2006 & 2007 & 2004 & 2006 & 2007 & 2004 & 2006 & 2007 \\
\hline Part du soutien dans les recettes brutes ${ }^{1}$ & n.a. & n.a. & n.a. & n.a. & n.a. & n.a. & n.a. & n.a. & n.a. \\
\hline Tous paiements $^{2}$ & 17 & 18 & 16 & 35 & 35 & 32 & 12 & 12 & 11 \\
\hline Paiements du 1er pilier & 13 & 14 & 13 & 24 & 25 & 22 & 9 & 10 & 10 \\
\hline Paiements du 2ème pilier & 2 & 2 & 1 & 7 & 7 & 7 & 1 & 1 & 0 \\
\hline Autres paiements & 2 & 2 & 1 & 4 & 4 & 2 & 1 & 1 & 1 \\
\hline Soutien des prix du marché ${ }^{3}$ & n.a. & n.a. & n.a. & n.a. & n.a. & n.a. & n.a. & n.a. & n.a. \\
\hline Part du soutien dans le revenu agricole & n.a. & n.a. & n.a. & n.a. & n.a. & n.a. & n.a. & n.a. & n.a. \\
\hline
\end{tabular}




\begin{tabular}{|c|c|c|c|c|c|c|c|c|c|}
\hline \multirow{3}{*}{$\begin{array}{l}\text { Pays } \\
\text { Quartile } \\
\text { Année }\end{array}$} & \multicolumn{9}{|c|}{ Allemagne } \\
\hline & \multicolumn{3}{|c|}{ Toutes exploitations } & \multicolumn{3}{|c|}{ 1er quartile } & \multicolumn{3}{|c|}{ 4ème quartile } \\
\hline & 2004 & 2006 & 2007 & 2004 & 2006 & 2007 & 2004 & 2006 & 2007 \\
\hline Part du soutien dans les recettes brutes ${ }^{1}$ & 27 & 23 & 20 & 41 & 37 & 34 & 24 & 20 & 18 \\
\hline Tous paiements ${ }^{2}$ & 14 & 14 & 14 & 29 & 28 & 27 & 12 & 12 & 12 \\
\hline Paiements du 1er pilier & 10 & 11 & 11 & 18 & 18 & 18 & 10 & 10 & 10 \\
\hline Paiements du 2ème pilier & 2 & 2 & 2 & 9 & 9 & 8 & 1 & 1 & 1 \\
\hline Autres paiements & 1 & 1 & 1 & 2 & 1 & 1 & 1 & 1 & 1 \\
\hline Soutien des prix du marché ${ }^{3}$ & 14 & 9 & 6 & 12 & 9 & 7 & 12 & 8 & 6 \\
\hline Part du soutien dans le revenu agricole & 207 & 149 & 121 & 1162 & 493 & 272 & 208 & 148 & 121 \\
\hline Pays & \multicolumn{9}{|c|}{ Irlande } \\
\hline Quartile & \multicolumn{3}{|c|}{ Toutes exploitations } & \multicolumn{3}{|c|}{ 1er quartile } & \multicolumn{3}{|c|}{ 4ème quartile } \\
\hline Année & 2004 & 2006 & 2007 & 2004 & 2006 & 2007 & 2004 & 2006 & 2007 \\
\hline Part du soutien dans les recettes brutes ${ }^{1}$ & 42 & 42 & 38 & 73 & 69 & 70 & 34 & 33 & 29 \\
\hline Tous paiements $^{2}$ & 29 & 33 & 31 & 67 & 64 & 67 & 18 & 24 & 21 \\
\hline Paiements du 1er pilier & 20 & 23 & 21 & 38 & 33 & 33 & 15 & 19 & 17 \\
\hline Paiements du 2ème pilier & 8 & 10 & 10 & 29 & 31 & 34 & 3 & 5 & 4 \\
\hline Autres paiements & 0 & 0 & 0 & 0 & 0 & 0 & 0 & 0 & 0 \\
\hline Soutien des prix du marché ${ }^{3}$ & 14 & 9 & 7 & 6 & 5 & 3 & 16 & 10 & 8 \\
\hline Part du soutien dans le revenu agricole & 128 & 124 & 103 & 241 & 178 & 202 & 101 & 100 & 75 \\
\hline Pays & \multicolumn{9}{|c|}{ Italie } \\
\hline Quartile & \multicolumn{3}{|c|}{ Toutes exploitations } & \multicolumn{3}{|c|}{ 1er quartile } & \multicolumn{3}{|c|}{ 4ème quartile } \\
\hline Année & 2004 & 2006 & 2007 & 2004 & 2006 & 2007 & 2004 & 2006 & 2007 \\
\hline Part du soutien dans les recettes brutes ${ }^{1}$ & 13 & 13 & 14 & 24 & 25 & 22 & 10 & 11 & 11 \\
\hline Tous paiements $^{2}$ & 10 & 12 & 9 & 20 & 23 & 16 & 7 & 9 & 7 \\
\hline Paiements du 1er pilier & 9 & 10 & 8 & 17 & 16 & 13 & 6 & 8 & 6 \\
\hline Paiements du 2ème pilier & 1 & 2 & 1 & 3 & 7 & 2 & 1 & 1 & 1 \\
\hline Autres paiements & 0 & 0 & 0 & 1 & 1 & 0 & 0 & 0 & 0 \\
\hline Soutien des prix du marché ${ }^{3}$ & 3 & 2 & 5 & 3 & 2 & 6 & 3 & 2 & 4 \\
\hline Part du soutien dans le revenu agricole & -322 & -408 & -1228 & -119 & -118 & -213 & -10627 & 325 & 336 \\
\hline
\end{tabular}

Les exploitations incluses représentent au total $90 \%$ de la production agricole nationale.

Les limites des quartiles sont établies à partir de la production agricole brute.

1. Inclut les recettes tirées des ventes de produits et de services agricoles tels que le travail à façon ou la mise en location de terres, de quotas, de bâtiments ou de machines, entre autres.

2. Inclut les transferts budgétaires aux agriculteurs résultant de la politique agricole et des paiements des systèmes d'assurance, des programmes de conservation des terres aux États-Unis et des premier et second piliers de la Politique agricole commune de l'Union européenne.

3. Le soutien des prix du marché (SPM) est calculé en appliquant le ratio SPM de chaque produit de base, lorsqu'il existe dans la base de données des ESP de l'OCDE, aux recettes de l'exploitation tirées de ce produit. Le ratio SPM moyen de l'ensemble des produits de la base ESP est utilisé pour les recettes provenant de produits pour lesquels il n'existe pas de ratio SPM.

Source : tableaux transmis par les participants. 
80 - Distribution DU SOUTIEN ET DES REVENUS DANS L'AGRICULTURE

Tableau annexe C5. Ratio de la moyenne des exploitations regroupées par type d'exploitations, sur la moyenne de l'ensemble des exploitations, 2004, 2006, 2007

\begin{tabular}{|c|c|c|c|c|c|c|c|c|c|c|c|c|c|c|c|c|c|c|c|c|c|c|c|c|c|c|c|}
\hline \multirow{3}{*}{$\begin{array}{l}\text { Pays } \\
\text { Type d'exploitation } \\
\text { Année }\end{array}$} & \multicolumn{27}{|c|}{ Canada } \\
\hline & \multicolumn{3}{|c|}{ Grandes cultures $^{14}$} & \multicolumn{3}{|c|}{$\begin{array}{l}\text { Pépinières, serres et } \\
\text { floriculture }\end{array}$} & \multicolumn{3}{|c|}{ Fruits et légumes } & \multicolumn{3}{|c|}{ Lait } & \multicolumn{3}{|c|}{ Bovins } & \multicolumn{3}{|c|}{ Porcins } & \multicolumn{3}{|c|}{ Volaille/oeufs } & \multicolumn{3}{|c|}{ Ovins/caprins } & \multicolumn{3}{|c|}{ Autres } \\
\hline & 2004 & 2006 & 2007 & 2004 & 2006 & 2007 & 2004 & 2006 & 2007 & 2004 & 2006 & 2007 & 2004 & 2006 & 2007 & 2004 & 2006 & 2007 & 2004 & 2006 & 2007 & 2004 & 2006 & 2007 & 2004 & 2006 & 2007 \\
\hline Exploitations couvertes & 0.40 & 0.40 & 0.43 & 0.03 & 0.03 & 0.03 & 0.04 & 0.04 & 0.04 & 0.18 & 0.16 & 0.15 & 0.18 & 0.21 & 0.19 & 0.06 & 0.05 & 0.04 & 0.04 & 0.04 & 0.04 & n.a. & n.a. & n.a. & 0.08 & 0.08 & 0.08 \\
\hline Unité de travail (éq. temps plein) & n.a. & n.a. & n.a. & n.a. & n.a. & n.a. & n.a. & n.a. & n.a. & n.a. & n.a. & n.a. & n.a. & n.a. & n.a. & n.a. & n.a. & n.a. & n.a. & n.a. & n.a. & n.a. & n.a. & n.a. & n.a. & n.a. & n.a. \\
\hline Superficie agricole utilisée & 1.68 & 1.75 & 1.74 & 0.07 & 0.05 & 0.06 & 0.19 & 0.18 & 0.17 & 0.38 & 0.40 & 0.36 & 0.89 & 0.76 & 0.65 & 0.69 & 0.61 & 0.45 & 0.17 & 0.14 & 0.18 & n.a. & n.a. & n.a. & 0.46 & 0.51 & 0.41 \\
\hline Part en \% des exploit. couvertes & 40 & 40 & 43 & 3 & 3 & 3 & 4 & 4 & 4 & 18 & 16 & 15 & 18 & 21 & 19 & 6 & 5 & 4 & 4 & 4 & 4 & n.a. & n.a. & n.a. & 8 & 8 & 8 \\
\hline Part en \% des unités de travail & n.a. & n.a. & n.a. & n.a. & n.a. & n.a. & n.a. & n.a. & n.a. & n.a. & n.a. & n.a. & n.a. & n.a. & n.a. & n.a. & n.a. & n.a. & n.a. & n.a. & n.a. & n.a. & n.a. & n.a. & n.a. & n.a. & n.a. \\
\hline Part en \% de la superficie & 68 & 69 & 76 & 0 & 0 & 0 & 1 & 1 & 1 & 7 & 6 & 6 & 16 & 16 & 12 & 4 & 3 & 2 & 1 & 1 & 1 & n.a. & n.a. & n.a. & 4 & 4 & 3 \\
\hline \multicolumn{28}{|l|}{ Composantes du revenu } \\
\hline Production agricole brute ${ }^{1}$ & 0.62 & 0.59 & 0.66 & 2.65 & 2.86 & 2.56 & 1.05 & 1.01 & 1.02 & 0.82 & 0.88 & 0.88 & 1.01 & 1.10 & 1.13 & 2.53 & 2.28 & 2.24 & 2.23 & 2.12 & 2.14 & n.a. & n.a. & n.a. & 1.00 & 0.97 & 0.94 \\
\hline Soutien total & 0.56 & 0.70 & 0.56 & 0.42 & 0.66 & 0.65 & 0.44 & 0.55 & 0.56 & 2.43 & 2.61 & 2.69 & 0.98 & 0.50 & 0.62 & 0.92 & 0.96 & 1.88 & 0.85 & 1.46 & 1.28 & n.a. & n.a. & n.a. & 0.67 & 0.66 & 0.71 \\
\hline Tous paiements $^{2}$ & 0.97 & 1.30 & 0.99 & n.a. & 1.24 & 1.18 & 0.76 & 1.02 & 1.01 & 0.45 & 0.34 & 0.35 & 1.70 & 0.92 & 1.12 & 1.45 & 1.67 & 3.34 & 0.46 & 0.33 & 0.33 & n.a. & n.a. & n.a. & 0.94 & 0.93 & 1.01 \\
\hline Paiements du 1er pilier & n.a. & n.a. & n.a. & n.a. & n.a. & n.a. & n.a. & n.a. & n.a. & n.a. & n.a. & n.a. & n.a. & n.a. & n.a. & n.a. & n.a. & n.a. & n.a. & n.a. & n.a. & n.a. & n.a. & n.a. & n.a. & n.a. & n.a. \\
\hline Paiements du 2ème pilier & n.a. & n.a. & n.a. & n.a. & n.a. & n.a. & n.a. & n.a. & n.a. & n.a. & n.a. & n.a. & n.a. & n.a. & n.a. & n.a. & n.a. & n.a. & n.a. & n.a. & n.a. & n.a. & n.a. & n.a. & n.a. & n.a. & n.a. \\
\hline Autres paiements & 0.97 & 1.30 & 0.99 & n.a. & 1.24 & 1.18 & 0.76 & 1.02 & 1.01 & 0.45 & 0.34 & 0.35 & 1.70 & 0.92 & 1.12 & 1.45 & 1.67 & 3.34 & 0.46 & 0.33 & 0.33 & n.a. & n.a. & n.a. & 0.94 & 0.93 & 1.01 \\
\hline Soutien des prix du marché ${ }^{3}$ & 0.02 & 0.02 & 0.03 & n.a. & 0.00 & 0.00 & 0.02 & 0.02 & 0.01 & 5.06 & 5.17 & 5.50 & 0.02 & 0.02 & 0.02 & 0.22 & 0.16 & 0.12 & 1.37 & 2.73 & 2.42 & n.a. & n.a. & n.a. & 0.31 & 0.37 & 0.34 \\
\hline Recettes brutes ${ }^{4}$ & 0.65 & 0.65 & 0.69 & 2.42 & 2.72 & 2.46 & 1.03 & 1.02 & 1.02 & 0.79 & 0.83 & 0.84 & 1.07 & 1.09 & 1.13 & 2.43 & 2.23 & 2.32 & 2.08 & 1.96 & 2.00 & n.a. & n.a. & n.a. & 0.99 & 0.97 & 0.95 \\
\hline Dépenses d'exploitation & 0.63 & 0.62 & 0.64 & 2.64 & 2.83 & 2.65 & 1.05 & 0.99 & 1.00 & 0.71 & 0.73 & 0.76 & 1.13 & 1.18 & 1.23 & 2.60 & 2.41 & 2.55 & 2.13 & 1.98 & 2.07 & n.a. & n.a. & n.a. & 0.96 & 0.93 & 0.93 \\
\hline Revenu net d'exploitation ${ }^{5}$ & 0.82 & 0.85 & 0.96 & 1.14 & 2.04 & 1.39 & 0.91 & 1.21 & 1.12 & 1.23 & 1.48 & 1.33 & 0.71 & 0.48 & 0.56 & 1.48 & 1.05 & 1.02 & 1.79 & 1.82 & 1.66 & n.a. & n.a. & n.a. & 1.19 & 1.23 & 1.06 \\
\hline Amortissements $^{6}$ & 0.95 & 0.94 & 0.95 & 1.63 & 2.01 & 1.78 & 0.78 & 0.75 & 0.82 & 1.15 & 1.24 & 1.23 & 0.65 & 0.64 & 0.64 & 1.60 & 1.52 & 1.48 & 1.30 & 1.34 & 1.33 & n.a. & n.a. & n.a. & 1.03 & 1.02 & 1.02 \\
\hline Revenu agricole $^{7}$ & 0.59 & 0.65 & 0.97 & 0.29 & 2.12 & 0.86 & 1.14 & 2.15 & 1.53 & 1.35 & 1.98 & 1.45 & 0.81 & 0.16 & 0.45 & 1.28 & 0.07 & 0.40 & 2.63 & 2.80 & 2.10 & n.a. & n.a. & n.a. & 1.47 & 1.65 & 1.12 \\
\hline Revenu non agricole ${ }^{8}$ & 1.13 & 1.11 & 1.07 & 0.57 & 0.45 & 0.40 & 0.99 & 1.17 & 0.95 & 0.64 & 0.61 & 0.58 & 1.24 & 1.21 & 1.45 & 0.60 & 0.52 & 0.45 & 0.61 & 0.57 & 0.56 & n.a. & n.a. & n.a. & 1.22 & 1.38 & 1.13 \\
\hline Revenu des ménages agricoles ${ }^{9}$ & 0.87 & 0.92 & 1.02 & 0.44 & 1.14 & 0.63 & 1.06 & 1.57 & 1.24 & 0.98 & 1.17 & 1.02 & 1.04 & 0.77 & 0.94 & 0.93 & 0.33 & 0.43 & 1.58 & 1.49 & 1.34 & n.a. & n.a. & n.a. & 1.34 & 1.49 & 1.13 \\
\hline Total des actifs agricoles ${ }^{11}$ & 0.81 & 0.87 & 0.91 & 1.02 & 0.92 & 0.96 & 0.84 & 0.87 & 0.90 & 1.59 & 1.63 & 1.54 & 0.83 & 0.77 & 0.72 & 1.18 & 1.16 & 0.99 & 1.50 & 1.52 & 1.71 & n.a. & п.а. & n.a. & 0.69 & 0.67 & 0.75 \\
\hline Total des dettes agricoles ${ }^{12}$ & 0.70 & 0.78 & 0.72 & 1.46 & 1.51 & 1.59 & 0.95 & 0.85 & 1.00 & 1.69 & 1.81 & 1.89 & 0.80 & 0.68 & 0.69 & 1.61 & 1.59 & 1.70 & 1.38 & 1.36 & 1.73 & n.a. & n.a. & n.a. & 0.65 & 0.60 & 0.50 \\
\hline Capitaux propres agricoles ${ }^{13}$ & 0.85 & 0.89 & 0.96 & 0.89 & 0.76 & 0.79 & 0.80 & 0.88 & 0.88 & 1.55 & 1.58 & 1.45 & 0.84 & 0.80 & 0.73 & 1.05 & 1.04 & 0.80 & 1.54 & 1.56 & 1.70 & n.a. & n.a. & n.a. & 0.71 & 0.69 & 0.81 \\
\hline
\end{tabular}




\begin{tabular}{|c|c|c|c|c|c|c|c|c|c|c|c|c|c|c|c|c|c|c|c|c|c|c|c|c|c|c|c|}
\hline \multirow{3}{*}{$\begin{array}{l}\text { Pays } \\
\text { Type d'exploitation } \\
\text { Année }\end{array}$} & \multicolumn{27}{|c|}{ États-Unis } \\
\hline & \multicolumn{3}{|c|}{ Grandes cultures $^{14}$} & \multicolumn{3}{|c|}{$\begin{array}{l}\text { Pépinières, serres et } \\
\text { floriculture }\end{array}$} & \multicolumn{3}{|c|}{ Fruits et légumes } & \multicolumn{3}{|c|}{ Lait } & \multicolumn{3}{|c|}{ Bovins } & \multicolumn{3}{|c|}{ Porcins } & \multicolumn{3}{|c|}{ Volaille/oeufs } & \multicolumn{3}{|c|}{ Ovins/caprins } & \multicolumn{3}{|c|}{ Autres } \\
\hline & 2004 & 2006 & 2007 & 2004 & 2006 & 2007 & 2004 & 2006 & 2007 & 2004 & 2006 & 2007 & 2004 & 2006 & 2007 & 2004 & 2006 & 2007 & 2004 & 2006 & 2007 & 2004 & 2006 & 2007 & 2004 & 2006 & 2007 \\
\hline Exploitations couvertes & 0.43 & 0.41 & 0.47 & 0.03 & 0.04 & 0.03 & 0.08 & 0.07 & 0.07 & 0.13 & 0.13 & 0.13 & 0.20 & 0.21 & 0.15 & 0.03 & 0.03 & 0.02 & 0.04 & 0.04 & 0.05 & 0.00 & 0.00 & 0.00 & 0.06 & 0.07 & 0.07 \\
\hline Unité de travail (éq. temps plein) & n.a. & n.a. & n.a. & n.a. & n.a. & n.a. & n.a. & n.a. & n.a. & n.a. & n.a. & n.a. & n.a. & n.a. & n.a. & n.a. & n.a. & n.a. & n.a. & n.a. & n.a. & n.a. & n.a. & n.a. & n.a. & n.a. & n.a. \\
\hline Superficie agricole utilisée & 0.83 & 0.92 & 0.96 & 0.07 & 0.07 & 0.12 & 0.24 & 0.32 & 0.36 & 0.27 & 0.30 & 0.31 & 2.65 & 2.38 & 2.84 & 0.41 & 0.40 & 0.43 & 0.13 & 0.16 & 0.14 & 3.40 & 3.15 & 2.82 & 0.55 & 0.67 & 0.43 \\
\hline Part en \% des exploit. couvertes & 43 & 41 & 47 & 3 & 4 & 3 & 8 & 7 & 7 & 13 & 13 & 13 & 20 & 21 & 15 & 3 & 3 & 2 & 4 & 4 & 5 & 0.3 & 0.2 & 0.5 & 6 & 7 & 7 \\
\hline Part en \% des unités de travail & n.a. & n.a. & n.a. & n.a. & n.a. & п.а. & n.a. & n.a. & n.a. & n.a. & n.a. & n.a. & n.a. & n.a. & n.a. & n.a. & n.a. & n.a. & n.a. & п.а. & n.a. & n.a. & n.a. & n.a. & n.a. & n.a. & n.a. \\
\hline Part en \% de la superficie & 35 & 38 & 45 & 0 & 0 & 0 & 2 & 2 & 3 & 3 & 4 & 4 & 53 & 49 & 42 & 1 & 1 & 1 & 1 & 1 & 1 & 1 & 1 & 1 & 3 & 5 & 3 \\
\hline \multicolumn{28}{|l|}{ Composantes du revenu } \\
\hline Production agricole brute ${ }^{1}$ & 0.73 & 0.74 & 0.73 & 1.87 & 1.96 & 2.37 & 1.70 & 2.21 & 1.71 & 1.22 & 1.21 & 1.27 & 0.96 & 0.83 & 1.00 & 2.10 & 1.75 & 2.23 & 0.98 & 0.63 & 0.84 & 0.45 & 0.84 & 0.38 & 0.74 & 0.81 & 0.66 \\
\hline Soutien total & 0.95 & 1.24 & 0.76 & 0.03 & 0.08 & 0.06 & 0.14 & 0.23 & 0.32 & 3.46 & 2.38 & 3.92 & 0.34 & 0.42 & 0.32 & 0.48 & 0.55 & 0.36 & 0.09 & 0.09 & 0.06 & 0.27 & 0.33 & 0.50 & 0.90 & 0.73 & 0.76 \\
\hline Tous paiements ${ }^{2}$ & 1.70 & 1.70 & 1.57 & 0.05 & 0.11 & 0.13 & 0.25 & 0.29 & 0.52 & 0.52 & 0.75 & 0.49 & 0.58 & 0.57 & 0.61 & 0.93 & 0.75 & 0.65 & 0.17 & 0.13 & 0.13 & 0.51 & 0.46 & 0.32 & 0.60 & 0.49 & 0.61 \\
\hline Paiements du 1er pilier & n.a. & n.a. & n.a. & n.a. & n.a. & n.a. & n.a. & n.a. & n.a. & n.a. & n.a. & n.a. & n.a. & n.a. & n.a. & n.a. & n.a. & n.a. & n.a. & n.a. & n.a. & n.a. & n.a. & n.a. & n.a. & n.a. & n.a. \\
\hline Paiements du zème pilier & n.a. & n.a. & n.a. & n.a. & n.a. & n.a. & n.a. & n.a. & n.a. & n.a. & n.a. & n.a. & n.a. & n.a. & n.a. & n.a. & n.a. & n.a. & n.a. & n.a. & n.a. & n.a. & n.a. & n.a. & n.a. & n.a. & n.a. \\
\hline Autres paiements & n.a. & n.a. & n.a. & n.a. & n.a. & п.а. & n.a. & n.a. & n.a. & n.a. & n.a. & n.a. & n.a. & n.a. & n.a. & n.a. & n.a. & n.a. & n.a. & п.а. & n.a. & n.a. & n.a. & n.a. & n.a. & n.a. & n.a. \\
\hline Soutien des prix du marché ${ }^{3}$ & 0.20 & 0.06 & 0.07 & 0.01 & 0.01 & 0.00 & 0.04 & 0.10 & 0.14 & 6.40 & 6.52 & 6.86 & 0.10 & 0.05 & 0.06 & 0.03 & 0.04 & 0.10 & 0.01 & 0.00 & 0.00 & 0.04 & 0.00 & 0.65 & 1.20 & 1.34 & 0.89 \\
\hline Recettes brutes ${ }^{4}$ & 0.77 & 0.78 & 0.75 & 1.79 & 1.87 & 2.30 & 1.64 & 2.13 & 1.67 & 1.19 & 1.19 & 1.25 & 0.94 & 0.82 & 0.99 & 2.05 & 1.70 & 2.18 & 0.95 & 0.61 & 0.82 & 0.46 & 0.82 & 0.38 & 0.73 & 0.79 & 0.66 \\
\hline Dépenses d'exploitation & 0.74 & 0.78 & 0.73 & 1.89 & 1.77 & 2.24 & 1.71 & 2.13 & 1.65 & 1.20 & 1.24 & 1.22 & 1.02 & 0.85 & 1.15 & 1.68 & 1.56 & 2.13 & 0.88 & 0.56 & 0.69 & 0.43 & 0.70 & 0.40 & 0.75 & 0.74 & 0.68 \\
\hline Revenu net d'exploitation ${ }^{5}$ & 0.84 & 0.78 & 0.81 & 1.58 & 2.22 & 2.45 & 1.47 & 2.12 & 1.73 & 1.16 & 1.03 & 1.30 & 0.77 & 0.71 & 0.58 & 2.93 & 2.16 & 2.30 & 1.10 & 0.76 & 1.16 & 0.53 & 1.22 & 0.32 & 0.68 & 0.95 & 0.60 \\
\hline Amortissements ${ }^{6}$ & 0.89 & 0.90 & 0.90 & 0.92 & 0.99 & 0.98 & 1.16 & 1.25 & 1.08 & 1.50 & 1.47 & 1.55 & 0.82 & 0.78 & 0.90 & 1.81 & 1.63 & 1.78 & 1.06 & 1.03 & 1.06 & 0.36 & 0.81 & 0.28 & 0.79 & 0.83 & 0.58 \\
\hline Revenu agricole $^{7}$ & 0.82 & 0.73 & 0.79 & 1.80 & 2.73 & 2.87 & 1.58 & 2.49 & 1.92 & 1.05 & 0.85 & 1.23 & 0.75 & 0.69 & 0.49 & 3.30 & 2.38 & 2.45 & 1.11 & 0.64 & 1.19 & 0.59 & 1.39 & 0.33 & 0.65 & 1.00 & 0.60 \\
\hline Revenu non agricole ${ }^{8}$ & 0.96 & 0.91 & 0.91 & 0.61 & 0.72 & 0.88 & 1.54 & 1.39 & 1.33 & 0.57 & 0.50 & 0.42 & 1.14 & 1.29 & 1.28 & 0.79 & 0.85 & 0.74 & 0.90 & 0.82 & 0.89 & 1.00 & 1.70 & 1.67 & 1.48 & 1.54 & 1.96 \\
\hline Revenu des ménages agricoles ${ }^{9}$ & 0.86 & 0.80 & 0.82 & 1.43 & 1.91 & 2.35 & 1.57 & 2.04 & 1.76 & 0.90 & 0.71 & 1.02 & 0.87 & 0.93 & 0.70 & 2.52 & 1.75 & 2.01 & 1.04 & 0.71 & 1.11 & 0.72 & 1.52 & 0.68 & 0.90 & 1.22 & 1.02 \\
\hline Total des actifs agricoles ${ }^{11}$ & 0.79 & 0.79 & 0.86 & 0.92 & 0.87 & 0.93 & 1.56 & 1.69 & 1.65 & 0.97 & 0.92 & 0.98 & 1.33 & 1.32 & 1.25 & 1.15 & 0.98 & 1.19 & 0.69 & 0.68 & 0.67 & 0.79 & 0.94 & 0.94 & 0.96 & 1.07 & 0.96 \\
\hline Total des dettes agricoles ${ }^{12}$ & 0.86 & 0.88 & 0.83 & 0.78 & 0.72 & 0.93 & 1.30 & 1.44 & 1.30 & 1.36 & 1.38 & 1.39 & 0.89 & 0.94 & 1.02 & 2.08 & 1.50 & 1.78 & 1.24 & 1.09 & 1.25 & 0.46 & 0.68 & 0.33 & 0.67 & 0.68 & 0.73 \\
\hline Capitaux propres agricoles ${ }^{13}$ & 0.78 & 0.78 & 0.87 & 0.94 & 0.89 & 0.93 & 1.60 & 1.72 & 1.70 & 0.90 & 0.86 & 0.93 & 1.39 & 1.37 & 1.28 & 1.00 & 0.92 & 1.11 & 0.61 & 0.63 & 0.59 & 0.84 & 0.97 & 1.02 & 1.00 & 1.13 & 1.00 \\
\hline
\end{tabular}


82 - Distribution DU SOUTIEN ET DES REVENUS DANS L'AGRICULTURE

\begin{tabular}{|c|c|c|c|c|c|c|c|c|c|c|c|c|c|c|c|c|c|c|c|c|c|c|c|c|c|c|c|}
\hline \multirow{3}{*}{$\begin{array}{l}\text { Pays } \\
\text { Type d'exploitation } \\
\text { Année }\end{array}$} & \multicolumn{27}{|c|}{ UE27 } \\
\hline & \multicolumn{3}{|c|}{ Grandes cultures ${ }^{14}$} & \multicolumn{3}{|c|}{$\begin{array}{l}\text { Pépinières, serres et } \\
\text { floriculture }\end{array}$} & \multicolumn{3}{|c|}{ Fruits et légumes } & \multicolumn{3}{|c|}{ Lait } & \multicolumn{3}{|c|}{ Bovins } & \multicolumn{3}{|c|}{ Porcins } & \multicolumn{3}{|c|}{ Volaille/oeufs } & \multicolumn{3}{|c|}{ Ovins/caprins } & \multicolumn{3}{|c|}{ Autres } \\
\hline & 2004 & 2006 & 2007 & 2004 & 2006 & 2007 & 2004 & 2006 & 2007 & 2004 & 2006 & 2007 & 2004 & 2006 & 2007 & 2004 & 2006 & 2007 & 2004 & 2006 & 2007 & 2004 & 2006 & 2007 & 2004 & 2006 & 2007 \\
\hline Exploitations couvertes & 0.27 & 0.27 & 0.28 & 0.03 & 0.03 & 0.03 & 0.21 & 0.23 & 0.19 & 0.13 & 0.13 & 0.15 & 0.08 & 0.08 & 0.08 & 0.02 & 0.02 & 0.02 & 0.01 & 0.01 & 0.01 & 0.04 & 0.04 & 0.04 & 0.21 & 0.20 & 0.22 \\
\hline Unité de travail (éq. temps plein) & 0.88 & 0.89 & 0.94 & 2.16 & 2.09 & 1.96 & 0.86 & 0.84 & 0.81 & 1.12 & 1.17 & 1.10 & 0.87 & 0.87 & 0.93 & 1.17 & 1.20 & 1.08 & 1.62 & 1.57 & 1.60 & 0.89 & 0.92 & 1.00 & 1.09 & 1.08 & 1.04 \\
\hline Superficie agricole utilisée & 1.41 & 1.44 & 1.44 & 0.09 & 0.09 & 0.10 & 0.24 & 0.24 & 0.28 & 1.28 & 1.28 & 1.08 & 1.38 & 1.43 & 1.46 & 0.71 & 0.73 & 0.69 & 0.36 & 0.42 & 0.35 & 2.00 & 1.96 & 1.82 & 0.91 & 0.92 & 0.86 \\
\hline Part en \% des exploit. couvertes & 27 & 27 & 28 & 3 & 3 & 3 & 21 & 23 & 19 & 13 & 13 & 15 & 8 & 8 & 8 & 2 & 2 & 2 & 1 & 1 & 1 & 4 & 4 & 4 & 21 & 20 & 22 \\
\hline Part en \% des unités de travail & 24 & 24 & 26 & 6 & 6 & 5 & 18 & 19 & 15 & 15 & 15 & 16 & 7 & 7 & 7 & 3 & 3 & 2 & 1 & 1 & 1 & 3 & 3 & 4 & 23 & 22 & 22 \\
\hline Part en \% de la superficie & 39 & 38 & 40 & 0 & 0 & 0 & 5 & 5 & 5 & 17 & 17 & 16 & 11 & 12 & 11 & 2 & 2 & 2 & 0 & 0 & 0 & 7 & 7 & 7 & 19 & 19 & 19 \\
\hline \multicolumn{28}{|l|}{ Composantes du revenu } \\
\hline Production agricole brute $^{1}$ & 0.86 & 0.87 & 0.92 & 2.75 & 2.90 & 2.75 & 0.48 & 0.46 & 0.53 & 1.51 & 1.51 & 1.36 & 0.63 & 0.73 & 0.69 & 2.92 & 3.20 & 2.64 & 4.44 & 4.33 & 4.56 & 0.71 & 0.72 & 0.66 & 1.00 & 1.00 & 0.94 \\
\hline Soutien total & 0.99 & 1.04 & 1.22 & 0.20 & 0.25 & 0.41 & 0.16 & 0.18 & 0.21 & 2.27 & 2.09 & 1.44 & 1.45 & 1.85 & 1.88 & 1.58 & 1.03 & 0.55 & 4.01 & 2.71 & 4.09 & 1.10 & 1.31 & 1.30 & 0.83 & 0.82 & 0.78 \\
\hline Tous paiements $^{2}$ & 1.36 & 1.34 & 1.34 & 0.13 & 0.16 & 0.17 & 0.28 & 0.25 & 0.26 & 1.40 & 1.59 & 1.38 & 1.67 & 1.68 & 1.73 & 0.68 & 0.70 & 0.68 & 0.56 & 0.62 & 0.66 & 1.57 & 1.47 & 1.37 & 0.82 & 0.81 & 0.78 \\
\hline Paiements du 1er pilier & 1.47 & 1.44 & 1.43 & 0.13 & 0.17 & 0.19 & 0.30 & 0.28 & 0.27 & 1.24 & 1.50 & 1.31 & 1.57 & 1.52 & 1.55 & 0.72 & 0.71 & 0.70 & 0.57 & 0.65 & 0.71 & 1.33 & 1.24 & 1.14 & 0.83 & 0.82 & 0.79 \\
\hline Paiements du Zème pilier & 0.79 & 0.88 & 0.89 & 0.09 & 0.09 & 0.11 & 0.15 & 0.15 & 0.19 & 2.27 & 2.01 & 1.68 & 2.25 & 2.39 & 2.55 & 0.50 & 0.68 & 0.56 & 0.49 & 0.50 & 0.40 & 2.82 & 2.52 & 2.48 & 0.75 & 0.80 & 0.75 \\
\hline Autres paiements & n.a. & n.a. & n.a. & n.a. & n.a. & n.a. & n.a. & n.a. & n.a. & n.a. & n.a. & n.a. & n.a. & n.a. & n.a. & n.a. & n.a. & n.a. & n.a. & n.a. & n.a. & n.a. & n.a. & n.a. & n.a. & n.a. & n.a. \\
\hline Soutien des prix du marché ${ }^{3}$ & 0.57 & 0.48 & 0.96 & 0.28 & 0.43 & 0.96 & 0.03 & 0.04 & 0.09 & 3.25 & 3.03 & 1.59 & 1.20 & 2.16 & 2.23 & 2.60 & 1.65 & 0.24 & 7.91 & 6.68 & 12.08 & 0.57 & 1.01 & 1.15 & 0.83 & 0.82 & 0.77 \\
\hline Recettes brutes ${ }^{4}$ & 0.93 & 0.94 & 0.98 & 2.37 & 2.47 & 2.39 & 0.45 & 0.43 & 0.49 & 1.50 & 1.53 & 1.36 & 0.78 & 0.88 & 0.84 & 2.59 & 2.81 & 2.37 & 3.87 & 3.74 & 4.01 & 0.84 & 0.84 & 0.76 & 0.98 & 0.97 & 0.92 \\
\hline Dépenses d'exploitation & 0.95 & 0.95 & 0.93 & 2.63 & 2.81 & 2.82 & 0.34 & 0.33 & 0.38 & 1.50 & 1.53 & 1.35 & 0.73 & 0.83 & 0.82 & 3.06 & 3.21 & 3.07 & 4.94 & 4.68 & 5.19 & 0.71 & 0.73 & 0.70 & 0.98 & 0.99 & 0.95 \\
\hline Revenu net d'exploitation ${ }^{5}$ & 0.91 & 0.93 & 1.05 & 1.94 & 1.91 & 1.73 & 0.64 & 0.59 & 0.66 & 1.49 & 1.53 & 1.38 & 0.86 & 0.96 & 0.86 & 1.82 & 2.16 & 1.26 & 2.08 & 2.19 & 2.17 & 1.06 & 1.02 & 0.85 & 0.96 & 0.93 & 0.88 \\
\hline Amortissements ${ }^{6}$ & 1.02 & 1.01 & 1.00 & 1.96 & 2.08 & 2.18 & 0.40 & 0.39 & 0.47 & 1.66 & 1.64 & 1.43 & 0.85 & 1.00 & 1.03 & 1.75 & 1.76 & 1.74 & 1.96 & 2.31 & 2.25 & 0.69 & 0.75 & 0.70 & 1.03 & 1.03 & 0.96 \\
\hline Revenu agricole $^{7}$ & 0.86 & 0.90 & 1.08 & 1.94 & 1.84 & 1.55 & 0.75 & 0.67 & 0.74 & 1.42 & 1.47 & 1.37 & 0.87 & 0.95 & 0.80 & 1.85 & 2.33 & 1.08 & 2.13 & 2.14 & 2.14 & 1.23 & 1.13 & 0.91 & 0.93 & 0.89 & 0.85 \\
\hline Revenu non agricole ${ }^{8}$ & n.a. & n.a. & n.a. & n.a. & n.a. & n.a. & n.a. & n.a. & n.a. & n.a. & n.a. & n.a. & n.a. & n.a. & n.a. & n.a. & n.a. & n.a. & n.a. & n.a. & n.a. & n.a. & n.a. & n.a. & n.a. & n.a. & n.a. \\
\hline Revenu des ménages agricoles ${ }^{9}$ & n.a. & n.a. & n.a. & n.a. & n.a. & n.a. & n.a. & n.a. & n.a. & n.a. & n.a. & n.a. & n.a. & n.a. & n.a. & n.a. & n.a. & n.a. & n.a. & n.a. & n.a. & n.a. & n.a. & n.a. & n.a. & n.a. & n.a. \\
\hline Total des actifs agricoles ${ }^{11}$ & 1.02 & 1.03 & 0.99 & 1.11 & 1.13 & 1.20 & 0.52 & 0.53 & 0.67 & 1.75 & 1.61 & 1.38 & 1.11 & 1.32 & 1.37 & 1.45 & 1.57 & 1.50 & 1.43 & 1.45 & 1.43 & 0.93 & 1.02 & 1.02 & 0.89 & 0.86 & 0.81 \\
\hline Total des dettes agricoles ${ }^{12}$ & 0.96 & 0.97 & 0.91 & 2.42 & 2.71 & 2.94 & 0.18 & 0.17 & 0.20 & 2.08 & 2.01 & 1.76 & 0.68 & 0.79 & 0.79 & 2.77 & 2.90 & 3.18 & 2.73 & 3.09 & 3.13 & 0.43 & 0.45 & 0.46 & 0.97 & 0.99 & 0.94 \\
\hline Capitaux propres agricoles ${ }^{13}$ & 1.03 & 1.04 & 1.00 & 0.87 & 0.86 & 0.92 & 0.58 & 0.60 & 0.75 & 1.69 & 1.55 & 1.32 & 1.19 & 1.41 & 1.47 & 1.21 & 1.34 & 1.22 & 1.19 & 1.17 & 1.15 & 1.02 & 1.12 & 1.11 & 0.88 & 0.83 & 0.79 \\
\hline
\end{tabular}




\begin{tabular}{|c|c|c|c|c|c|c|c|c|c|c|c|c|c|c|c|c|c|c|c|c|c|c|c|c|c|c|c|}
\hline \multirow{3}{*}{$\begin{array}{l}\text { Pays } \\
\text { Type d'exploitation } \\
\text { Année }\end{array}$} & \multicolumn{27}{|c|}{ Danemark } \\
\hline & \multicolumn{3}{|c|}{ Grandes cultures $^{14}$} & \multicolumn{3}{|c|}{$\begin{array}{l}\text { Pépinières, serres et } \\
\text { floriculture }\end{array}$} & \multicolumn{3}{|c|}{ Fruits et légumes } & \multicolumn{3}{|c|}{ Lait } & \multicolumn{3}{|c|}{ Bovins } & \multicolumn{3}{|c|}{ Porcins } & \multicolumn{3}{|c|}{ Volaille/oeufs } & \multicolumn{3}{|c|}{ Ovins/caprins } & \multicolumn{3}{|c|}{ Autres } \\
\hline & 2004 & 2006 & 2007 & 2004 & 2006 & 2007 & 2004 & 2006 & 2007 & 2004 & 2006 & 2007 & 2004 & 2006 & 2007 & 2004 & 2006 & 2007 & 2004 & 2006 & 2007 & 2004 & 2006 & 2007 & 2004 & 2006 & 2007 \\
\hline Exploitations couvertes & 0.16 & 0.13 & 0.15 & n.a. & n.a. & n.a. & n.a. & n.a. & n.a. & 0.31 & 0.34 & 0.35 & n.a. & n.a. & n.a. & 0.16 & 0.12 & 0.14 & 0.31 & 0.35 & 0.31 & n.a. & n.a. & n.a. & 0.06 & 0.05 & 0.04 \\
\hline Unité de travail (éq. temps plein) & 0.88 & 0.90 & 0.86 & n.a. & n.a. & n.a. & n.a. & n.a. & n.a. & 0.95 & 0.80 & 0.79 & n.a. & n.a. & n.a. & 1.32 & 1.14 & 1.16 & 0.94 & 1.08 & 1.11 & n.a. & n.a. & n.a. & 1.05 & 0.93 & 0.99 \\
\hline Superficie agricole utilisée & 1.55 & 2.00 & 1.77 & n.a. & n.a. & n.a. & n.a. & n.a. & n.a. & 0.88 & 0.88 & 0.84 & n.a. & n.a. & n.a. & 0.82 & 0.83 & 0.83 & 0.87 & 0.83 & 0.88 & n.a. & n.a. & n.a. & 1.26 & 1.19 & 1.35 \\
\hline Part en \% des exploit. couvertes & 16 & 13 & 15 & n.a. & n.a. & n.a. & n.a. & n.a. & n.a. & 31 & 34 & 35 & n.a. & n.a. & n.a. & 16 & 12 & 14 & 31 & 35 & 31 & n.a. & n.a. & n.a. & 6 & 5 & 4 \\
\hline Part en \% des unités de travail & 14 & 11 & 13 & n.a. & n.a. & n.a. & n.a. & n.a. & n.a. & 29 & 27 & 27 & n.a. & n.a. & n.a. & 21 & 14 & 16 & 29 & 38 & 34 & n.a. & n.a. & n.a. & 7 & 4 & 4 \\
\hline Part en \% de la superficie & 25 & 25 & 27 & n.a. & n.a. & n.a. & n.a. & n.a. & n.a. & 27 & 30 & 29 & n.a. & n.a. & n.a. & 13 & 10 & 11 & 27 & 29 & 27 & n.a. & n.a. & n.a. & 8 & 5 & 5 \\
\hline \multicolumn{28}{|l|}{ Composantes du revenu } \\
\hline Production agricole brute ${ }^{1}$ & 0.92 & 0.91 & 0.93 & n.a. & n.a. & n.a. & n.a. & n.a. & n.a. & 0.81 & 0.79 & 0.87 & n.a. & n.a. & n.a. & 1.38 & 1.30 & 1.13 & 1.05 & 1.09 & 1.08 & n.a. & n.a. & n.a. & 0.94 & 1.06 & 1.10 \\
\hline Soutien total & 0.69 & 0.71 & 0.60 & n.a. & n.a. & n.a. & n.a. & n.a. & n.a. & 1.03 & 1.14 & 2.84 & n.a. & n.a. & n.a. & 1.22 & 1.20 & 1.04 & 0.96 & 0.80 & 0.78 & n.a. & n.a. & n.a. & 1.12 & 1.40 & 1.22 \\
\hline Tous paiements $^{2}$ & 1.47 & 1.70 & 1.50 & n.a. & n.a. & n.a. & n.a. & n.a. & n.a. & 0.94 & 1.13 & 1.11 & n.a. & n.a. & n.a. & 0.73 & 0.63 & 0.65 & 0.79 & 0.64 & 0.71 & n.a. & n.a. & n.a. & 1.82 & 2.03 & 1.92 \\
\hline Paiements du 1er pilier & n.a. & n.a. & n.a. & n.a. & n.a. & n.a. & n.a. & n.a. & n.a. & n.a. & n.a. & n.a. & n.a. & n.a. & n.a. & n.a. & n.a. & n.a. & n.a. & n.a. & n.a. & n.a. & n.a. & n.a. & n.a. & n.a. & n.a. \\
\hline Paiements du zème pilier & n.a. & n.a. & n.a. & n.a. & n.a. & n.a. & n.a. & n.a. & n.a. & n.a. & n.a. & n.a. & n.a. & n.a. & n.a. & n.a. & n.a. & n.a. & n.a. & n.a. & n.a. & n.a. & n.a. & n.a. & n.a. & n.a. & n.a. \\
\hline Autres paiements & n.a. & n.a. & n.a. & n.a. & n.a. & n.a. & n.a. & n.a. & n.a. & n.a. & n.a. & n.a. & n.a. & n.a. & n.a. & n.a. & n.a. & n.a. & n.a. & n.a. & n.a. & n.a. & n.a. & n.a. & n.a. & n.a. & n.a. \\
\hline Soutien des prix du marché ${ }^{3}$ & 0.32 & 0.22 & 0.05 & n.a. & n.a. & n.a. & n.a. & n.a. & n.a. & 1.07 & 1.15 & 3.89 & n.a. & n.a. & n.a. & 1.45 & 1.48 & 1.28 & 1.04 & 0.87 & 0.83 & n.a. & n.a. & n.a. & 0.79 & 1.09 & 0.79 \\
\hline Recettes brutes ${ }^{4}$ & 0.97 & 0.98 & 0.98 & n.a. & n.a. & n.a. & n.a. & n.a. & n.a. & 0.82 & 0.81 & 0.89 & n.a. & n.a. & n.a. & 1.32 & 1.25 & 1.10 & 1.03 & 1.05 & 1.05 & n.a. & n.a. & n.a. & 1.03 & 1.14 & 1.16 \\
\hline Dépenses d'exploitation & 0.83 & 0.90 & 0.85 & n.a. & n.a. & n.a. & n.a. & n.a. & n.a. & 0.76 & 0.79 & 0.77 & n.a. & n.a. & n.a. & 1.52 & 1.29 & 1.36 & 1.08 & 1.09 & 1.14 & n.a. & n.a. & n.a. & 0.94 & 1.07 & 1.05 \\
\hline Revenu net d'exploitation ${ }^{5}$ & 1.32 & 1.22 & 1.58 & n.a. & n.a. & n.a. & n.a. & n.a. & n.a. & 0.97 & 0.90 & 1.47 & n.a. & n.a. & n.a. & 0.81 & 1.10 & -0.21 & 0.90 & 0.93 & 0.61 & n.a. & п.а. & n.a. & 1.26 & 1.36 & 1.74 \\
\hline Amortissements ${ }^{6}$ & 0.92 & 1.13 & 1.05 & n.a. & n.a. & n.a. & n.a. & n.a. & n.a. & 0.81 & 0.77 & 0.75 & n.a. & n.a. & n.a. & 1.43 & 1.34 & 1.37 & 1.02 & 1.07 & 1.09 & n.a. & n.a. & n.a. & 0.96 & 0.90 & 1.06 \\
\hline Revenu agricole $^{7}$ & 1.59 & 1.29 & 2.47 & n.a. & n.a. & n.a. & n.a. & n.a. & n.a. & 1.08 & 1.01 & 2.66 & n.a. & n.a. & n.a. & 0.40 & 0.92 & -2.81 & 0.83 & 0.82 & -0.16 & n.a. & n.a. & n.a. & 1.46 & 1.71 & 2.86 \\
\hline Revenu non agricole ${ }^{8}$ & 1.14 & 1.39 & 1.39 & n.a. & n.a. & n.a. & n.a. & n.a. & n.a. & 0.77 & 0.79 & 0.76 & n.a. & n.a. & n.a. & 0.95 & 1.13 & 1.01 & 1.16 & 1.04 & 1.09 & n.a. & n.a. & n.a. & 1.11 & 0.90 & 0.87 \\
\hline Revenu des ménages agricoles ${ }^{9}$ & 1.48 & 1.32 & 2.07 & n.a. & n.a. & n.a. & n.a. & n.a. & n.a. & 1.00 & 0.96 & 1.96 & n.a. & n.a. & n.a. & 0.53 & 0.97 & -1.39 & 0.90 & 0.87 & 0.30 & n.a. & n.a. & n.a. & 1.38 & 1.53 & 2.12 \\
\hline Total des actifs agricoles ${ }^{11}$ & n.a. & n.a. & n.a. & n.a. & n.a. & n.a. & n.a. & n.a. & n.a. & n.a. & n.a. & n.a. & n.a. & n.a. & n.a. & n.a. & n.a. & n.a. & n.a. & n.a. & n.a. & n.a. & n.a. & n.a. & n.a. & n.a. & n.a. \\
\hline Total des dettes agricoles ${ }^{12}$ & n.a. & n.a. & n.a. & n.a. & n.a. & n.a. & n.a. & n.a. & n.a. & n.a. & n.a. & n.a. & n.a. & n.a. & n.a. & n.a. & n.a. & n.a. & n.a. & n.a. & n.a. & n.a. & n.a. & n.a. & n.a. & n.a. & n.a. \\
\hline Capitaux propres agricoles ${ }^{13}$ & n.a. & n.a. & n.a. & n.a. & n.a. & n.a. & n.a. & n.a. & n.a. & n.a. & n.a. & n.a. & n.a. & n.a. & n.a. & n.a. & n.a. & n.a. & n.a. & n.a. & n.a. & n.a. & n.a. & n.a. & n.a. & n.a. & n.a. \\
\hline
\end{tabular}


84 - Distribution DU SOUTIEN ET DES REVENUS DANS L'AGRICULTURE

\begin{tabular}{|c|c|c|c|c|c|c|c|c|c|c|c|c|c|c|c|c|c|c|c|c|c|c|c|c|c|c|c|}
\hline \multirow{3}{*}{$\begin{array}{l}\text { Pays } \\
\text { Type d'exploitation } \\
\text { Année }\end{array}$} & \multicolumn{27}{|c|}{ Angleterre } \\
\hline & \multicolumn{3}{|c|}{ Grandes cultures $^{14}$} & \multicolumn{3}{|c|}{$\begin{array}{l}\text { Pépinières, serres et } \\
\text { floriculture }\end{array}$} & \multicolumn{3}{|c|}{ Fruits et légumes } & \multicolumn{3}{|c|}{ Lait } & \multicolumn{3}{|c|}{ Bovins } & \multicolumn{3}{|c|}{ Porcins } & \multicolumn{3}{|c|}{ Volaille/oeufs } & \multicolumn{3}{|c|}{ Ovins/caprins } & \multicolumn{3}{|c|}{ Autres } \\
\hline & 2004 & 2006 & 2007 & 2004 & 2006 & 2007 & 2004 & 2006 & 2007 & 2004 & 2006 & 2007 & 2004 & 2006 & 2007 & 2004 & 2006 & 2007 & 2004 & 2006 & 2007 & 2004 & 2006 & 2007 & 2004 & 2006 & 2007 \\
\hline Exploitations couvertes & 0.10 & 0.10 & 0.09 & 0.04 & 0.04 & 0.04 & 0.02 & 0.02 & 0.02 & 0.20 & 0.19 & 0.16 & 0.10 & 0.10 & 0.12 & 0.02 & 0.02 & 0.02 & 0.03 & 0.02 & 0.01 & 0.11 & 0.12 & 0.13 & 0.37 & 0.39 & 0.40 \\
\hline Unité de travail (éq. temps plein) & 1.32 & 1.09 & 1.15 & 2.66 & 2.46 & 2.70 & 1.45 & 1.88 & 1.72 & 1.00 & 1.01 & 1.13 & 0.51 & 0.48 & 0.48 & 1.29 & 1.21 & 1.40 & 1.55 & 1.57 & 1.91 & 0.53 & 0.51 & 0.51 & 0.92 & 1.03 & 0.98 \\
\hline Superficie agricole utilisée & 1.76 & 1.55 & 1.71 & 0.07 & 0.08 & 0.10 & 0.29 & 0.50 & 0.40 & 0.69 & 0.77 & 0.84 & 0.64 & 0.68 & 0.66 & 0.29 & 0.24 & 0.28 & 0.16 & 0.18 & 0.18 & 1.02 & 0.93 & 0.87 & 1.30 & 1.27 & 1.23 \\
\hline Part en \% des exploit. couvertes & 10 & 10 & 9 & 4 & 4 & 4 & 2 & 2 & 2 & 20 & 19 & 16 & 10 & 10 & 12 & 2 & 2 & 2 & 3 & 2 & 1 & 11 & 12 & 13 & 37 & 39 & 40 \\
\hline Part en \% des unités de travail & 13 & 11 & 11 & 12 & 10 & 11 & 3 & 4 & 3 & 20 & 19 & 18 & 5 & 5 & 6 & 2 & 2 & 2 & 4 & 3 & 3 & 6 & 6 & 7 & 34 & 40 & 39 \\
\hline Part en \% de la superficie & 17 & 15 & 16 & 0 & 0 & 0 & 1 & 1 & 1 & 14 & 15 & 14 & 7 & 7 & 8 & 0 & 0 & 0 & 0 & 0 & 0 & 12 & 11 & 12 & 48 & 49 & 49 \\
\hline \multicolumn{28}{|l|}{ Composantes du revenu } \\
\hline Production agricole brute ${ }^{1}$ & 1.62 & 1.50 & 1.54 & 2.56 & 2.10 & 2.11 & 0.98 & 1.17 & 1.11 & 1.05 & 1.03 & 1.28 & 0.26 & 0.27 & 0.24 & 2.12 & 1.72 & 1.79 & 3.83 & 3.33 & 3.62 & 0.27 & 0.24 & 0.22 & 0.80 & 1.01 & 1.01 \\
\hline Soutien total & 1.63 & 1.27 & 1.28 & 0.08 & 0.08 & 0.10 & 0.07 & 0.14 & 0.15 & 0.99 & 1.34 & 1.68 & 0.59 & 0.76 & 0.69 & 1.22 & 1.34 & 1.51 & 1.68 & 2.13 & 1.89 & 0.54 & 0.62 & 0.61 & 1.20 & 1.01 & 0.96 \\
\hline Tous paiements $^{2}$ & 1.85 & 1.54 & 1.68 & 0.02 & 0.03 & 0.04 & 0.08 & 0.24 & 0.24 & 0.49 & 0.81 & 0.86 & 0.55 & 0.79 & 0.71 & 0.22 & 0.15 & 0.20 & 0.10 & 0.12 & 0.16 & 0.60 & 0.75 & 0.77 & 1.57 & 1.32 & 1.26 \\
\hline Paiements du 1er pilier & 1.94 & 1.62 & 1.72 & 0.02 & 0.02 & 0.03 & 0.07 & 0.21 & 0.19 & 0.48 & 0.83 & 0.92 & 0.51 & 0.75 & 0.67 & 0.23 & 0.15 & 0.20 & 0.11 & 0.13 & 0.16 & 0.42 & 0.63 & 0.65 & 1.62 & 1.33 & 1.28 \\
\hline Paiements du Zème pilier & 0.88 & 1.15 & 1.60 & 0.08 & 0.06 & 0.08 & 0.22 & 0.38 & 0.43 & 0.51 & 0.62 & 0.57 & 1.06 & 0.97 & 0.81 & 0.08 & 0.11 & 0.17 & 0.06 & 0.09 & 0.17 & 2.60 & 1.35 & 1.24 & 1.05 & 1.27 & 1.19 \\
\hline Autres paiements & 0.26 & 0.29 & 0.04 & 0.00 & 0.00 & 0.00 & 0.04 & 0.00 & 0.00 & 1.69 & 3.68 & 2.29 & 0.67 & 0.78 & 2.27 & 0.01 & 0.00 & 0.00 & 0.04 & 0.00 & 0.04 & 2.51 & 0.84 & 0.43 & 0.73 & 0.21 & 0.73 \\
\hline Soutien des prix du marché ${ }^{3}$ & 1.35 & 1.00 & 0.90 & 0.16 & 0.14 & 0.15 & 0.05 & 0.05 & 0.06 & 1.64 & 1.88 & 2.46 & 0.65 & 0.73 & 0.68 & 2.53 & 2.52 & 2.76 & 3.76 & 4.14 & 3.53 & 0.47 & 0.50 & 0.45 & 0.71 & 0.70 & 0.68 \\
\hline Recettes brutes ${ }^{4}$ & 1.67 & 1.50 & 1.56 & 1.99 & 1.79 & 1.83 & 0.78 & 1.03 & 0.99 & 0.93 & 1.00 & 1.23 & 0.33 & 0.35 & 0.31 & 1.69 & 1.48 & 1.58 & 3.00 & 2.84 & 3.15 & 0.34 & 0.32 & 0.29 & 0.97 & 1.06 & 1.04 \\
\hline Dépenses d'exploitation & 1.65 & 1.38 & 1.47 & 2.21 & 1.99 & 2.09 & 0.90 & 1.16 & 1.04 & 0.88 & 1.03 & 1.26 & 0.31 & 0.33 & 0.30 & 1.92 & 1.73 & 2.07 & 3.21 & 2.99 & 3.43 & 0.30 & 0.30 & 0.30 & 0.96 & 1.03 & 0.99 \\
\hline Revenu net d'exploitation ${ }^{5}$ & 1.75 & 1.83 & 1.77 & 1.32 & 1.23 & 1.21 & 0.43 & 0.68 & 0.87 & 1.05 & 0.92 & 1.14 & 0.38 & 0.40 & 0.32 & 1.00 & 0.80 & 0.42 & 2.34 & 2.43 & 2.49 & 0.48 & 0.35 & 0.28 & 1.00 & 1.12 & 1.17 \\
\hline Amortissements ${ }^{6}$ & 1.99 & 1.67 & 1.75 & 0.89 & 1.09 & 0.99 & 0.67 & 0.82 & 0.79 & 0.85 & 0.96 & 1.13 & 0.48 & 0.51 & 0.44 & 1.14 & 0.98 & 1.16 & 1.14 & 1.46 & 1.48 & 0.42 & 0.41 & 0.40 & 1.16 & 1.14 & 1.13 \\
\hline Revenu agricole $^{7}$ & 1.62 & 1.91 & 1.78 & 1.57 & 1.30 & 1.29 & 0.29 & 0.61 & 0.91 & 1.17 & 0.90 & 1.15 & 0.32 & 0.34 & 0.27 & 0.91 & 0.71 & 0.13 & 3.04 & 2.93 & 2.89 & 0.52 & 0.33 & 0.23 & 0.91 & 1.11 & 1.18 \\
\hline Revenu non agricole ${ }^{8}$ & n.a. & n.a. & n.a. & n.a. & n.a. & n.a. & n.a. & n.a. & n.a. & n.a. & n.a. & n.a. & n.a. & n.a. & n.a. & n.a. & n.a. & n.a. & n.a. & n.a. & n.a. & n.a. & n.a. & n.a. & n.a. & n.a. & n.a. \\
\hline Revenu des ménages agricoles ${ }^{9}$ & n.a. & n.a. & n.a. & n.a. & n.a. & n.a. & n.a. & n.a. & n.a. & n.a. & n.a. & n.a. & n.a. & n.a. & n.a. & n.a. & n.a. & n.a. & n.a. & n.a. & n.a. & n.a. & n.a. & n.a. & n.a. & n.a. & n.a. \\
\hline Total des actifs agricoles ${ }^{11}$ & 1.46 & 1.43 & 1.48 & 0.80 & 0.60 & 0.57 & 0.66 & 0.66 & 0.75 & 0.91 & 0.90 & 1.00 & 0.67 & 0.69 & 0.70 & 0.68 & 0.61 & 0.56 & 0.66 & 0.65 & 0.67 & 0.54 & 0.59 & 0.57 & 1.24 & 1.24 & 1.21 \\
\hline Total des dettes agricoles ${ }^{12}$ & 1.52 & 1.41 & 1.49 & 1.09 & 1.05 & 1.18 & 0.57 & 0.93 & 0.90 & 1.18 & 1.25 & 1.45 & 0.55 & 0.55 & 0.43 & 1.68 & 1.60 & 1.89 & 1.66 & 1.82 & 2.24 & 0.28 & 0.29 & 0.32 & 1.05 & 1.04 & 1.01 \\
\hline Capitaux propres agricoles ${ }^{13}$ & 1.45 & 1.44 & 1.48 & 0.75 & 0.54 & 0.48 & 0.67 & 0.62 & 0.72 & 0.86 & 0.85 & 0.94 & 0.69 & 0.71 & 0.74 & 0.53 & 0.46 & 0.38 & 0.51 & 0.48 & 0.45 & 0.58 & 0.64 & 0.60 & 1.27 & 1.27 & 1.23 \\
\hline
\end{tabular}




\begin{tabular}{|c|c|c|c|c|c|c|c|c|c|c|c|c|c|c|c|c|c|c|c|c|c|c|c|c|c|c|c|}
\hline \multirow{3}{*}{$\begin{array}{l}\text { Pays } \\
\text { Type d'exploitation } \\
\text { Année }\end{array}$} & \multicolumn{27}{|c|}{ Espagne } \\
\hline & \multicolumn{3}{|c|}{ Grandes cultures $^{14}$} & \multicolumn{3}{|c|}{$\begin{array}{l}\text { Pépinières, serres et } \\
\text { floriculture }\end{array}$} & \multicolumn{3}{|c|}{ Fruits et légumes } & \multicolumn{3}{|c|}{ Lait } & \multicolumn{3}{|c|}{ Bovins } & \multicolumn{3}{|c|}{ Porcins } & \multicolumn{3}{|c|}{ Volaille/oeufs } & \multicolumn{3}{|c|}{ Ovins/caprins } & \multicolumn{3}{|c|}{ Autres } \\
\hline & 2004 & 2006 & 2007 & 2004 & 2006 & 2007 & 2004 & 2006 & 2007 & 2004 & 2006 & 2007 & 2004 & 2006 & 2007 & 2004 & 2006 & 2007 & 2004 & 2006 & 2007 & 2004 & 2006 & 2007 & 2004 & 2006 & 2007 \\
\hline Exploitations couvertes & 0.21 & 0.21 & 0.19 & 0.06 & 0.06 & 0.05 & 0.45 & 0.44 & 0.50 & 0.06 & 0.05 & 0.04 & 0.04 & 0.05 & 0.05 & 0.01 & 0.02 & 0.01 & 0.00 & 0.00 & 0.01 & 0.05 & 0.05 & 0.05 & 0.12 & 0.12 & 0.10 \\
\hline Unité de travail (éq. temps plein) & 0.73 & 0.80 & 0.90 & 2.00 & 1.60 & 2.30 & 1.00 & 0.80 & 0.90 & 1.09 & 1.00 & 1.60 & 1.00 & 1.00 & 1.00 & 1.45 & 1.80 & 1.90 & 0.82 & 0.90 & 1.20 & 1.09 & 1.10 & 1.30 & 1.18 & 1.30 & 1.40 \\
\hline Superficie agricole utilisée & 1.95 & 2.22 & 2.12 & 0.12 & 0.11 & 0.21 & 0.42 & 0.32 & 0.36 & 0.50 & 0.43 & 0.72 & 1.96 & 1.39 & 1.37 & 0.69 & 0.69 & 1.12 & 0.27 & 0.30 & 0.49 & 1.33 & 1.28 & 1.85 & 2.09 & 2.06 & 2.22 \\
\hline Part en \% des exploit. couvertes & 21 & 21 & 19 & 6 & 6 & 5 & 45 & 44 & 50 & 6 & 5 & 4 & 4 & 5 & 5 & 1 & 2 & 1 & 0 & 0 & 1 & 5 & 5 & 5 & 12 & 12 & 10 \\
\hline Part en \% des unités de travail & 15 & 17 & 17 & 11 & 10 & 11 & 45 & 35 & 45 & 6 & 5 & 6 & 4 & 5 & 5 & 2 & 3 & 2 & 0 & 0 & 1 & 6 & 5 & 7 & 14 & 16 & 14 \\
\hline Part en \%de la superficie & 41 & 47 & 41 & 1 & 1 & 1 & 19 & 14 & 18 & 3 & 2 & 3 & 7 & 7 & 6 & 1 & 1 & 1 & 0 & 0 & 0 & 7 & 6 & 10 & 24 & 25 & 22 \\
\hline \multicolumn{28}{|l|}{ Composantes du revenu } \\
\hline Production agricole brute ${ }^{1}$ & 0.84 & 0.96 & 0.96 & 2.15 & 1.46 & 1.99 & 0.65 & 0.57 & 0.54 & 1.20 & 1.31 & 2.46 & 0.81 & 0.86 & 0.83 & 5.94 & 6.39 & 6.10 & 2.38 & 3.71 & 3.22 & 1.34 & 1.44 & 1.61 & 1.48 & 1.61 & 1.63 \\
\hline Soutien total & n.a. & n.a. & n.a. & n.a. & n.a. & n.a. & n.a. & n.a. & n.a. & n.a. & n.a. & n.a. & n.a. & n.a. & n.a. & n.a. & n.a. & n.a. & n.a. & n.a. & n.a. & n.a. & n.a. & n.a. & n.a. & n.a. & n.a. \\
\hline Tous paiements $^{2}$ & 1.87 & 2.25 & 2.17 & 0.03 & 0.05 & 0.17 & 0.52 & 0.35 & 0.22 & 0.59 & 0.70 & 1.67 & 2.05 & 1.37 & 1.99 & 0.62 & 0.57 & 1.04 & 0.46 & 0.46 & 0.62 & 1.41 & 1.33 & 2.13 & 1.78 & 1.78 & 2.06 \\
\hline Paiements du 1er pilier & n.a. & n.a. & n.a. & n.a. & n.a. & n.a. & n.a. & n.a. & n.a. & n.a. & n.a. & n.a. & n.a. & n.a. & n.a. & n.a. & n.a. & n.a. & n.a. & n.a. & n.a. & n.a. & n.a. & n.a. & n.a. & n.a. & n.a. \\
\hline Paiements du 2ème pilier & n.a. & n.a. & n.a. & n.a. & n.a. & n.a. & n.a. & n.a. & n.a. & n.a. & n.a. & n.a. & n.a. & n.a. & n.a. & n.a. & n.a. & n.a. & n.a. & n.a. & n.a. & n.a. & n.a. & n.a. & n.a. & n.a. & n.a. \\
\hline Autres paiements & n.a. & n.a. & n.a. & n.a. & n.a. & n.a. & n.a. & n.a. & n.a. & n.a. & n.a. & n.a. & n.a. & n.a. & n.a. & n.a. & n.a. & n.a. & n.a. & n.a. & n.a. & n.a. & n.a. & n.a. & n.a. & n.a. & n.a. \\
\hline Soutien des prix du marché ${ }^{3}$ & n.a. & n.a. & n.a. & n.a. & n.a. & n.a. & n.a. & n.a. & n.a. & n.a. & n.a. & n.a. & n.a. & n.a. & n.a. & n.a. & n.a. & n.a. & n.a. & n.a. & n.a. & n.a. & n.a. & n.a. & n.a. & n.a. & n.a. \\
\hline Recettes brutes ${ }^{4}$ & 0.99 & 1.17 & 1.10 & 1.84 & 1.22 & 1.78 & 0.63 & 0.53 & 0.50 & 1.11 & 1.21 & 2.36 & 0.99 & 0.95 & 0.97 & 5.16 & 5.41 & 5.51 & 2.10 & 3.16 & 2.92 & 1.35 & 1.42 & 1.67 & 1.52 & 1.64 & 1.68 \\
\hline Dépenses d'exploitation & 0.97 & 1.21 & 1.00 & 1.93 & 1.20 & 2.02 & 0.53 & 0.45 & 0.45 & 1.22 & 1.26 & 2.56 & 1.09 & 1.00 & 1.06 & 7.66 & 7.41 & 7.35 & 2.56 & 4.41 & 3.87 & 1.29 & 1.35 & 1.75 & 1.50 & 1.60 & 1.66 \\
\hline Revenu net d'exploitation ${ }^{5}$ & 1.00 & 1.15 & 1.19 & 1.76 & 1.24 & 1.57 & 0.71 & 0.60 & 0.55 & 1.02 & 1.17 & 2.20 & 0.92 & 0.90 & 0.89 & 3.04 & 3.68 & 3.96 & 1.70 & 2.08 & 2.12 & 1.40 & 1.48 & 1.61 & 1.54 & 1.67 & 1.70 \\
\hline Amortissements ${ }^{6}$ & 0.96 & 1.13 & 1.19 & 1.40 & 0.98 & 1.66 & 0.82 & 0.79 & 0.59 & 0.89 & 0.90 & 1.93 & 1.02 & 0.72 & 0.72 & 4.37 & 4.35 & 5.80 & 2.16 & 2.56 & 4.11 & 1.03 & 0.94 & 1.29 & 1.33 & 1.38 & 1.42 \\
\hline Revenu agricole $^{7}$ & 1.01 & 1.15 & 1.19 & 1.79 & 1.27 & 1.57 & 0.70 & 0.58 & 0.55 & 1.03 & 1.20 & 2.23 & 0.91 & 0.92 & 0.91 & 2.92 & 3.59 & 3.77 & 1.66 & 2.02 & 1.91 & 1.43 & 1.54 & 1.64 & 1.56 & 1.70 & 1.72 \\
\hline Revenu non agricole ${ }^{8}$ & n.a. & n.a. & n.a. & n.a. & n.a. & n.a. & n.a. & n.a. & n.a. & n.a. & n.a. & n.a. & n.a. & n.a. & n.a. & n.a. & n.a. & n.a. & n.a. & n.a. & n.a. & n.a. & n.a. & n.a. & n.a. & n.a. & n.a. \\
\hline Revenu des ménages agricoles ${ }^{9}$ & n.a. & n.a. & n.a. & n.a. & n.a. & n.a. & n.a. & n.a. & n.a. & n.a. & n.a. & n.a. & n.a. & n.a. & n.a. & n.a. & n.a. & n.a. & n.a. & n.a. & n.a. & n.a. & n.a. & n.a. & n.a. & n.a. & n.a. \\
\hline Total des actifs agricoles ${ }^{11}$ & 1.10 & 1.29 & 1.16 & 1.06 & 1.02 & 1.44 & 0.78 & 0.81 & 0.85 & 1.35 & 0.90 & 1.43 & 1.13 & 0.83 & 0.60 & 1.59 & 1.87 & 1.69 & 0.93 & 1.75 & 1.22 & 0.99 & 0.91 & 1.04 & 1.54 & 1.34 & 1.31 \\
\hline Total des dettes agricoles ${ }^{12}$ & 1.17 & 1.22 & 0.96 & 3.25 & 2.72 & 4.19 & 0.50 & 0.31 & 0.18 & 0.85 & 1.17 & 2.83 & 0.96 & 0.77 & 1.43 & 7.31 & 8.81 & 10.01 & 1.38 & 2.53 & 5.28 & 0.72 & 1.16 & 2.03 & 1.06 & 1.29 & 1.12 \\
\hline Capitaux propres agricoles ${ }^{13}$ & 1.09 & 1.29 & 1.17 & 0.99 & 0.98 & 1.39 & 0.79 & 0.82 & 0.86 & 1.36 & 0.90 & 1.40 & 1.13 & 0.83 & 0.58 & 1.42 & 1.74 & 1.55 & 0.92 & 1.73 & 1.15 & 1.00 & 0.91 & 1.03 & 1.55 & 1.34 & 1.31 \\
\hline
\end{tabular}


86 - Distribution DU SOUTIEN ET DES REVENUS DANS L'AGRICULTURE

\begin{tabular}{|c|c|c|c|c|c|c|c|c|c|c|c|c|c|c|c|c|c|c|c|c|c|c|c|c|c|c|c|}
\hline \multirow{3}{*}{$\begin{array}{l}\text { Pays } \\
\text { Type d'exploitation } \\
\text { Année }\end{array}$} & \multicolumn{27}{|c|}{ Estonie } \\
\hline & \multicolumn{3}{|c|}{ Grandes cultures $^{14}$} & \multicolumn{3}{|c|}{$\begin{array}{l}\text { Pépinières, serres et } \\
\text { flloriculture }\end{array}$} & \multicolumn{3}{|c|}{ Fruits et légumes } & \multicolumn{3}{|c|}{ Lait } & \multicolumn{3}{|c|}{ Bovins } & \multicolumn{3}{|c|}{ Porcins } & \multicolumn{3}{|c|}{ Volaille/oeufs } & \multicolumn{3}{|c|}{ Ovins'caprins } & \multicolumn{3}{|c|}{ Autres } \\
\hline & 2004 & 2006 & 2007 & 2004 & 2006 & 2007 & 2004 & 2006 & 2007 & 2004 & 2006 & 2007 & 2004 & 2006 & 2007 & 2004 & 2006 & 2007 & 2004 & 2006 & 2007 & 2004 & 2006 & 2007 & 2004 & 2006 & 2007 \\
\hline Exploitations couvertes & 0.39 & 0.43 & 0.43 & 0.01 & 0.02 & 0.01 & 0.03 & 0.03 & 0.04 & 0.25 & 0.18 & 0.18 & 0.03 & 0.04 & 0.03 & 0.01 & 0.01 & 0.01 & n.a. & n.a. & n.a. & 0.01 & 0.05 & 0.07 & 0.28 & 0.24 & 0.23 \\
\hline Unité de travail (éq. temps plein) & 0.53 & 0.59 & 0.65 & 2.42 & 2.90 & 1.41 & 2.20 & 0.91 & 0.80 & 1.68 & 1.55 & 2.00 & 0.48 & 0.68 & 0.52 & 3.29 & 4.07 & 3.31 & n.a. & n.a. & n.a. & n.a. & 0.62 & 0.65 & 0.84 & 1.13 & 0.99 \\
\hline Superficie agricole utilisée & 0.79 & 0.91 & 0.94 & 0.04 & 0.04 & 0.04 & 0.35 & 0.27 & 0.26 & 1.57 & 1.31 & 1.60 & 1.41 & 1.41 & 0.72 & 0.25 & 0.11 & 0.32 & n.a. & n.a. & n.a. & n.a. & 0.58 & 0.57 & 0.85 & 1.16 & 1.04 \\
\hline Part en \% des exploit. couvertes & 39 & 43 & 43 & 1 & 2 & 1 & 3 & 3 & 4 & 25 & 18 & 18 & 3 & 4 & 3 & 1 & 1 & 1 & n.a. & n.a. & n.a. & n.a. & 5 & 7 & 28 & 24 & 23 \\
\hline Part en $\%$ des unités de travail & 21 & 25 & 28 & 2 & 5 & 2 & 7 & 3 & 3 & 42 & 28 & 35 & 1 & 3 & 1 & 3 & 5 & 2 & n.a. & n.a. & n.a. & n.a. & 3 & 5 & 24 & 27 & 22 \\
\hline Part en \% de la superficie & 31 & 39 & 41 & 0 & 0 & 0 & 1 & 1 & 1 & 39 & 24 & 28 & 4 & 6 & 2 & 0 & 0 & 0 & n.a. & n.a. & n.a. & n.a. & 3 & 4 & 24 & 28 & 23 \\
\hline \multicolumn{28}{|l|}{ Composantes du revenu } \\
\hline Production agricole brute ${ }^{1}$ & 0.44 & 0.47 & 0.69 & 2.38 & 4.82 & 2.34 & 2.20 & 0.44 & 0.32 & 1.75 & 1.43 & 2.02 & 0.29 & 0.44 & 0.27 & 9.26 & 10.20 & 7.11 & ก.a. & n.a. & n.a. & n.a. & 0.23 & 0.19 & 0.71 & 1.14 & 0.95 \\
\hline Soutien total & 0.50 & 0.65 & 0.72 & 0.13 & 0.22 & 0.70 & 0.45 & 0.27 & 0.15 & 2.02 & 1.71 & 1.90 & 1.01 & 1.29 & 1.11 & 1.71 & 2.07 & 0.26 & n.a. & n.a. & n.a. & n.a. & 0.52 & 0.61 & 0.84 & 1.24 & 1.14 \\
\hline Tous paiements $^{2}$ & 0.76 & 0.84 & 0.78 & 0.00 & 0.00 & 0.58 & 0.50 & 0.33 & 0.13 & 1.56 & 1.39 & 1.78 & 1.51 & 1.37 & 1.12 & 0.07 & 0.90 & 0.61 & n.a. & n.a. & n.a. & n.a. & 0.62 & 0.62 & 0.87 & 1.17 & 1.12 \\
\hline Paiements du 1er pilier & 0.75 & 0.88 & 0.83 & 0.00 & 0.00 & 0.00 & 0.14 & 0.13 & 0.11 & 1.71 & 1.39 & 1.97 & 1.07 & 1.17 & 0.67 & 0.00 & 0.00 & 0.20 & ก.a. & n.a. & n.a. & n.a. & 0.47 & 0.40 & 0.86 & 1.24 & 1.05 \\
\hline Paiements du zème pilier & 0.76 & 0.76 & 0.71 & 0.00 & 0.00 & 1.46 & 0.78 & 0.20 & 0.16 & 1.49 & 1.46 & 1.49 & 1.88 & 1.74 & 1.81 & 0.12 & 2.11 & 1.22 & n.a. & n.a. & n.a. & n.a. & 0.82 & 0.95 & 0.85 & 1.12 & 1.23 \\
\hline Autres paiements & 1.10 & 1.23 & 0.00 & 0.00 & 0.00 & 0.00 & 0.00 & 5.46 & 0.00 & 0.37 & 0.49 & 2.82 & 0.00 & 0.00 & 0.00 & 0.00 & 0.00 & 0.00 & ก.a. & n.a. & n.a. & n.a. & 0.37 & 0.00 & 1.73 & 0.80 & 2.22 \\
\hline Soutien des prix du marché ${ }^{3}$ & 0.12 & 0.16 & 0.40 & 0.31 & 0.76 & 1.39 & 0.38 & 0.13 & 0.30 & 2.69 & 2.50 & 2.61 & 0.26 & 1.08 & 1.02 & 4.15 & 4.99 & -1.76 & n.a. & n.a. & n.a. & n.a. & 0.26 & 0.56 & 0.80 & 1.42 & 1.22 \\
\hline Recettes brutes ${ }^{4}$ & 0.50 & 0.54 & 0.71 & 1.90 & 3.87 & 2.00 & 1.86 & 0.42 & 0.28 & 1.71 & 1.42 & 1.98 & 0.54 & 0.62 & 0.44 & 7.41 & 8.37 & 5.85 & n.a. & n.a. & n.a. & n.a. & 0.31 & 0.27 & 0.74 & 1.15 & 0.99 \\
\hline Dépenses d'exploitation & 0.43 & 0.45 & 0.56 & 3.41 & 5.01 & 2.39 & 2.00 & 0.38 & 0.31 & 1.70 & 1.45 & 2.18 & 0.44 & 0.59 & 0.32 & 9.81 & 9.62 & 8.21 & n.a. & n.a. & n.a. & n.a. & 0.23 & 0.26 & 0.74 & 1.18 & 1.02 \\
\hline Revenu net d'exploitation ${ }^{5}$ & 0.66 & 0.81 & 1.01 & -1.32 & 0.47 & 1.20 & 1.54 & 0.54 & 0.23 & 1.74 & 1.35 & 1.55 & 0.75 & 0.71 & 0.67 & 2.29 & 4.64 & 1.07 & n.a. & n.a. & n.a. & n.a. & 0.52 & 0.30 & 0.75 & 1.07 & 0.92 \\
\hline Amortissements ${ }^{6}$ & 0.72 & 0.73 & 0.75 & 1.83 & 1.44 & 1.57 & 1.43 & 0.69 & 0.57 & 1.56 & 1.41 & 2.03 & 0.42 & 0.54 & 0.30 & 4.49 & 5.54 & 6.22 & n.a. & n.a. & n.a. & n.a. & 0.17 & 0.25 & 0.71 & 1.12 & 0.84 \\
\hline Revenu agricole ${ }^{7}$ & 0.64 & 0.86 & 1.11 & -2.46 & 0.00 & 1.06 & 1.58 & 0.46 & 0.09 & 1.81 & 1.32 & 1.37 & 0.86 & 0.78 & 0.81 & 1.50 & 4.21 & -0.94 & n.a. & n.a. & n.a. & n.a. & 0.70 & 0.31 & 0.77 & 1.05 & 0.96 \\
\hline Revenu non agricole ${ }^{8}$ & n.a. & n.a. & n.a. & n.a. & n.a. & n.a. & n.a. & n.a. & n.a. & n.a. & n.a. & n.a. & n.a. & n.a. & n.a. & n.a. & n.a. & n.a. & n.a. & n.a. & n.a. & n.a. & n.a. & n.a. & n.a. & n.a. & n.a. \\
\hline Revenu des ménages agricoles ${ }^{9}$ & ก.a. & n.a. & n.a. & n.a. & n.a. & ก.a. & n.a. & n.a. & n.a. & n.a. & n.a. & n.a. & n.a. & ก.a. & n.a. & n.a. & n.a. & n.a. & n.a. & n.a. & n.a. & n.a. & ก.a. & n.a. & ก.a. & n.a. & n.a. \\
\hline Total des actifs agricoles ${ }^{11}$ & 0.62 & 0.67 & 0.73 & 1.54 & 2.98 & 1.19 & 0.93 & 0.59 & 0.41 & 1.70 & 1.29 & 1.88 & 0.88 & 0.87 & 0.57 & 3.86 & 5.89 & 4.21 & n.a. & n.a. & n.a. & n.a. & 0.55 & 0.59 & 0.79 & 1.12 & 0.97 \\
\hline Total des dettes agricoles ${ }^{12}$ & 0.54 & 0.54 & 0.63 & 4.60 & 6.47 & 2.37 & 1.20 & 0.53 & 0.36 & 1.64 & 1.40 & 2.17 & 0.68 & 0.58 & 0.65 & 9.96 & 2.81 & 3.36 & n.a. & n.a. & n.a. & n.a. & 0.30 & 0.37 & 0.68 & 1.27 & 0.99 \\
\hline Capitaux propres agricoles ${ }^{13}$ & 0.64 & 0.72 & 0.77 & 0.69 & 1.63 & 0.75 & 0.85 & 0.61 & 0.43 & 1.72 & 1.25 & 1.77 & 0.93 & 0.98 & 0.54 & 2.15 & 7.08 & 4.53 & п.а. & n.a. & n.a. & n.a. & 0.65 & 0.67 & 0.82 & 1.06 & 0.97 \\
\hline
\end{tabular}




\begin{tabular}{|c|c|c|c|c|c|c|c|c|c|c|c|c|c|c|c|c|c|c|c|c|c|c|c|c|c|c|c|}
\hline \multirow{3}{*}{$\begin{array}{l}\text { Pays } \\
\text { Type d'exploitation } \\
\text { Année }\end{array}$} & \multicolumn{27}{|c|}{ Flandre belge } \\
\hline & \multicolumn{3}{|c|}{ Grandes cultures ${ }^{14}$} & \multicolumn{3}{|c|}{$\begin{array}{l}\text { Pépinières, serres et } \\
\text { floriculture }\end{array}$} & \multicolumn{3}{|c|}{ Fruits et légumes } & \multicolumn{3}{|c|}{ Lait } & \multicolumn{3}{|c|}{ Bovins } & \multicolumn{3}{|c|}{ Porcins } & \multicolumn{3}{|c|}{ Volaille/oeufs } & \multicolumn{3}{|c|}{ Ovins/caprins } & \multicolumn{3}{|c|}{ Autres } \\
\hline & 2004 & 2006 & 2007 & 2004 & 2006 & 2007 & 2004 & 2006 & 2007 & 2004 & 2006 & 2007 & 2004 & 2006 & 2007 & 2004 & 2006 & 2007 & 2004 & 2006 & 2007 & 2004 & 2006 & 2007 & 2004 & 2006 & 2007 \\
\hline Exploitations couvertes & 0.11 & 0.12 & 0.12 & 0.12 & 0.12 & 0.12 & 0.10 & 0.09 & 0.09 & 0.21 & 0.19 & 0.18 & 0.14 & 0.15 & 0.17 & 0.12 & 0.13 & 0.12 & n.a. & ก.a. & n.a. & n.a. & n.a. & n.a. & 0.20 & 0.20 & 0.20 \\
\hline Unité de travail (éq. temps plein) & 0.80 & 0.76 & 0.72 & 1.69 & 1.69 & 1.74 & 1.77 & 1.94 & 1.97 & 0.76 & 0.73 & 0.74 & 0.76 & 0.76 & 0.74 & 0.75 & 0.75 & 0.73 & n.a. & n.a. & n.a. & n.a. & n.a. & n.a. & 0.88 & 0.88 & 0.88 \\
\hline Superficie agricole utilisée & 1.58 & 1.48 & 1.49 & 0.06 & 0.06 & 0.05 & 0.56 & 0.54 & 0.52 & 1.20 & 1.19 & 1.15 & 1.40 & 1.34 & 1.30 & 0.53 & 0.47 & 0.49 & n.a. & n.a. & n.a. & n.a. & n.a. & n.a. & 1.26 & 1.40 & 1.40 \\
\hline Part en \% des exploit. couvertes & 11 & 12 & 12 & 12 & 12 & 12 & 10 & 9 & 9 & 21 & 19 & 18 & 14 & 15 & 17 & 12 & 13 & 12 & n.a. & n.a. & n.a. & n.a. & n.a. & n.a. & 20 & 20 & 20 \\
\hline Part en \% des unités de travail & 9 & 9 & 9 & 21 & 20 & 21 & 17 & 18 & 19 & 16 & 14 & 13 & 11 & 12 & 12 & 9 & 10 & 9 & n.a. & n.a. & n.a. & n.a. & n.a. & n.a. & 18 & 18 & 18 \\
\hline Part en \% de la superficie & 18 & 18 & 18 & 1 & 1 & 1 & 5 & 5 & 5 & 25 & 22 & 21 & 20 & 21 & 22 & 6 & 6 & 6 & n.a. & n.a. & n.a. & n.a. & n.a. & n.a. & 25 & 28 & 28 \\
\hline \multicolumn{28}{|l|}{ Composantes du revenu } \\
\hline Production agricole brute ${ }^{1}$ & 0.77 & 0.65 & 0.67 & 1.28 & 1.49 & 1.38 & 1.08 & 1.22 & 1.31 & 0.64 & 0.61 & 0.72 & 0.63 & 0.62 & 0.63 & 1.86 & 1.72 & 1.66 & n.a. & n.a. & n.a. & n.a. & n.a. & n.a. & 1.05 & 1.00 & 1.01 \\
\hline Soutien total & 0.95 & 0.84 & 1.29 & 0.19 & 0.27 & 0.51 & 0.26 & 0.28 & 0.34 & 1.24 & 1.28 & 1.03 & 1.44 & 1.63 & 1.62 & 1.22 & 0.71 & 0.41 & n.a. & n.a. & n.a. & n.a. & n.a. & n.a. & 1.21 & 1.31 & 1.22 \\
\hline Tous paiements $^{2}$ & 1.13 & 1.08 & 1.16 & 0.25 & 0.26 & 0.29 & 0.34 & 0.22 & 0.25 & 1.05 & 1.31 & 1.21 & 2.01 & 1.61 & 1.42 & 0.45 & 0.42 & 0.52 & n.a. & n.a. & n.a. & n.a. & n.a. & n.a. & 1.27 & 1.38 & 1.42 \\
\hline Paiements du 1er pilier & 0.99 & 1.02 & 1.10 & 0.01 & 0.00 & 0.00 & 0.09 & 0.08 & 0.09 & 1.13 & 1.44 & 1.36 & 2.36 & 1.79 & 1.68 & 0.41 & 0.31 & 0.33 & n.a. & n.a. & n.a. & n.a. & n.a. & n.a. & 1.31 & 1.45 & 1.46 \\
\hline Paiements du 2ème pilier & 1.00 & 1.26 & 1.18 & 1.28 & 1.29 & 0.99 & 1.50 & 0.83 & 0.70 & 0.90 & 0.92 & 0.83 & 0.83 & 0.87 & 0.72 & 0.50 & 0.90 & 1.12 & n.a. & n.a. & n.a. & n.a. & n.a. & n.a. & 1.10 & 0.99 & 1.36 \\
\hline Autres paiements & 4.71 & 1.45 & 2.15 & 0.34 & 0.95 & 1.82 & 0.17 & 0.45 & 0.69 & 0.20 & 0.55 & 0.56 & 0.18 & 1.20 & 0.55 & 1.10 & 0.52 & 0.58 & n.a. & n.a. & n.a. & n.a. & n.a. & n.a. & 1.09 & 1.60 & 0.99 \\
\hline Soutien des prix du marché ${ }^{3}$ & 0.89 & 0.68 & 1.42 & 0.17 & 0.27 & 0.71 & 0.23 & 0.32 & 0.43 & 1.30 & 1.26 & 0.87 & 1.26 & 1.65 & 1.81 & 1.47 & 0.91 & 0.31 & n.a. & n.a. & n.a. & n.a. & n.a. & n.a. & 1.19 & 1.27 & 1.03 \\
\hline Recettes brutes ${ }^{4}$ & 0.79 & 0.68 & 0.71 & 1.23 & 1.41 & 1.30 & 1.04 & 1.15 & 1.24 & 0.67 & 0.66 & 0.75 & 0.70 & 0.69 & 0.68 & 1.79 & 1.63 & 1.58 & n.a. & n.a. & n.a. & n.a. & n.a. & n.a. & 1.06 & 1.03 & 1.04 \\
\hline Dépenses d'exploitation & 0.69 & 0.58 & 0.63 & 1.38 & 1.58 & 1.43 & 0.93 & 1.09 & 1.01 & 0.56 & 0.56 & 0.58 & 0.70 & 0.64 & 0.66 & 1.97 & 1.86 & 2.03 & n.a. & n.a. & n.a. & n.a. & n.a. & n.a. & 1.08 & 0.99 & 1.02 \\
\hline Revenu net d'exploitation ${ }^{5}$ & 0.98 & 0.84 & 0.83 & 0.94 & 1.13 & 1.08 & 1.26 & 1.26 & 1.64 & 0.86 & 0.83 & 1.06 & 0.70 & 0.75 & 0.72 & 1.43 & 1.25 & 0.78 & n.a. & n.a. & n.a. & n.a. & n.a. & n.a. & 1.02 & 1.09 & 1.07 \\
\hline Amortissements $^{6}$ & 0.70 & 0.71 & 0.67 & 1.48 & 1.61 & 1.63 & 1.28 & 1.46 & 1.40 & 0.90 & 0.75 & 0.79 & 0.67 & 0.72 & 0.67 & 1.05 & 1.07 & 1.20 & n.a. & n.a. & n.a. & n.a. & n.a. & n.a. & 1.04 & 0.99 & 1.00 \\
\hline Revenu agricole $^{7}$ & 1.12 & 0.89 & 0.90 & 0.67 & 0.95 & 0.84 & 1.26 & 1.19 & 1.74 & 0.84 & 0.86 & 1.17 & 0.71 & 0.76 & 0.74 & 1.62 & 1.32 & 0.60 & n.a. & n.a. & n.a. & n.a. & n.a. & n.a. & 1.01 & 1.12 & 1.10 \\
\hline Revenu non agricole ${ }^{8}$ & n.a. & n.a. & n.a. & n.a. & n.a. & n.a. & n.a. & n.a. & n.a. & n.a. & n.a. & n.a. & n.a. & n.a. & n.a. & n.a. & n.a. & n.a. & n.a. & n.a. & n.a. & n.a. & n.a. & n.a. & n.a. & n.a. & n.a. \\
\hline Revenu des ménages agricoles ${ }^{9}$ & n.a. & n.a. & n.a. & n.a. & n.a. & n.a. & n.a. & n.a. & n.a. & n.a. & n.a. & n.a.. & n.a. & n.a. & n.a. & n.a. & n.a. & n.a. & n.a. & n.a. & n.a. & n.a. & n.a. & n.a. & n.a. & n.a. & n.a. \\
\hline Total des actifs agricoles ${ }^{11}$ & 0.87 & 0.78 & 0.75 & 0.86 & 0.79 & 0.82 & 1.08 & 1.05 & 0.99 & 0.96 & 1.10 & 1.08 & 1.02 & 1.01 & 0.94 & 0.95 & 0.89 & 0.98 & n.a. & n.a. & n.a. & n.a. & n.a. & n.a. & 1.18 & 1.21 & 1.26 \\
\hline Total des dettes agricoles ${ }^{12}$ & 0.74 & 0.67 & 0.61 & 1.34 & 1.33 & 1.42 & 0.98 & 0.95 & 0.83 & 0.81 & 0.91 & 0.87 & 0.90 & 0.82 & 0.78 & 1.30 & 1.22 & 1.44 & n.a. & n.a. & n.a. & n.a. & n.a. & n.a. & 1.04 & 1.11 & 1.11 \\
\hline Capitaux propres agricoles ${ }^{13}$ & 0.93 & 0.82 & 0.81 & 0.65 & 0.58 & 0.59 & 1.13 & 1.09 & 1.04 & 1.02 & 1.17 & 1.15 & 1.07 & 1.08 & 1.00 & 0.79 & 0.77 & 0.80 & n.a. & n.a. & n.a. & n.a. & n.a. & n.a. & 1.24 & 1.25 & 1.32 \\
\hline
\end{tabular}


88 - Distribution DU SOUTIEN ET DES REVENUS DANS L'AGRICULTURE

\begin{tabular}{|c|c|c|c|c|c|c|c|c|c|c|c|c|c|c|c|c|c|c|c|c|c|c|c|c|c|c|c|}
\hline \multirow{3}{*}{$\begin{array}{l}\text { Pays } \\
\text { Type d'exploitation } \\
\text { Année }\end{array}$} & \multicolumn{27}{|c|}{ Pays-Bas } \\
\hline & \multicolumn{3}{|c|}{ Grandes cultures $^{14}$} & \multicolumn{3}{|c|}{$\begin{array}{l}\text { Pépinières, serres et } \\
\text { floriculture }\end{array}$} & \multicolumn{3}{|c|}{ Fruits et légumes } & \multicolumn{3}{|c|}{ Lait } & \multicolumn{3}{|c|}{ Bovins } & \multicolumn{3}{|c|}{ Porcins } & \multicolumn{3}{|c|}{ Volaille/oeufs } & \multicolumn{3}{|c|}{ Ovins/caprins } & \multicolumn{3}{|c|}{ Autres } \\
\hline & 2004 & 2006 & 2007 & 2004 & 2006 & 2007 & 2004 & 2006 & 2007 & 2004 & 2006 & 2007 & 2004 & 2006 & 2007 & 2004 & 2006 & 2007 & 2004 & 2006 & 2007 & 2004 & 2006 & 2007 & 2004 & 2006 & 2007 \\
\hline Exploitations couvertes & 0.16 & 0.16 & 0.15 & 0.13 & 0.13 & 0.13 & 0.04 & 0.04 & 0.04 & 0.34 & 0.32 & 0.32 & 0.14 & 0.15 & 0.16 & 0.06 & 0.06 & 0.07 & 0.02 & 0.02 & 0.02 & n.a. & n.a. & n.a. & 0.12 & 0.12 & 0.12 \\
\hline Unité de travail (éq. temps plein) & 0.69 & 0.71 & 0.71 & 2.76 & 2.40 & 2.50 & 1.40 & 1.71 & 1.84 & 0.66 & 0.72 & 0.69 & 0.52 & 0.64 & 0.61 & 0.63 & 0.70 & 0.68 & 0.71 & 0.80 & 0.65 & n.a. & n.a. & n.a. & 1.03 & 1.00 & 1.04 \\
\hline Superficie agricole utilisée & 1.71 & 1.66 & 1.67 & 0.11 & 0.13 & 0.16 & 0.50 & 0.45 & 0.49 & 1.35 & 1.33 & 1.36 & 0.77 & 0.92 & 0.86 & 0.28 & 0.28 & 0.28 & 0.22 & 0.19 & 0.16 & n.a. & n.a. & n.a. & 1.00 & 1.02 & 1.02 \\
\hline Part en \% des exploit. couvertes & 16 & 16 & 15 & 13 & 13 & 13 & 4 & 4 & 4 & 34 & 32 & 32 & 14 & 15 & 16 & 6 & 6 & 7 & 2 & 2 & 2 & n.a. & n.a. & n.a. & 12 & 12 & 12 \\
\hline Part en \% des unités de travail & 11 & 11 & 11 & 37 & 31 & 32 & 5 & 7 & 7 & 22 & 23 & 22 & 7 & 9 & 9 & 4 & 4 & 5 & 1 & 2 & 2 & n.a. & n.a. & n.a. & 12 & 12 & 12 \\
\hline Part en \% de la superficie & 27 & 26 & 26 & 2 & 2 & 2 & 2 & 2 & 2 & 46 & 43 & 43 & 11 & 14 & 13 & 2 & 2 & 2 & 0 & 0 & 0 & n.a. & n.a. & n.a. & 12 & 12 & 12 \\
\hline \multicolumn{28}{|l|}{ Composantes du revenu } \\
\hline Production agricole brute $^{1}$ & 0.69 & 0.78 & 0.69 & 2.19 & 2.16 & 2.28 & 0.90 & 1.08 & 1.14 & 0.74 & 0.69 & 0.75 & 0.31 & 0.39 & 0.35 & 1.72 & 1.61 & 1.30 & 1.95 & 1.95 & 2.10 & n.a. & n.a. & n.a. & 1.11 & 1.08 & 1.10 \\
\hline Soutien total & 0.84 & 0.82 & 1.05 & 0.25 & 0.29 & 0.72 & 0.15 & 0.14 & 0.39 & 1.54 & 1.53 & 1.24 & 0.68 & 0.98 & 0.94 & 1.30 & 0.64 & 0.02 & 1.79 & 1.55 & 2.52 & n.a. & n.a. & n.a. & 0.87 & 0.98 & 1.14 \\
\hline Tous paiements $^{2}$ & 1.39 & 1.16 & 1.25 & 0.11 & 0.19 & 0.42 & 0.15 & 0.10 & 0.11 & 1.30 & 1.63 & 1.56 & 1.65 & 1.00 & 0.95 & 0.27 & 0.16 & 0.17 & 0.20 & 0.18 & 0.14 & n.a. & n.a. & n.a. & 0.68 & 0.89 & 0.82 \\
\hline Paiements du 1er pilier & 1.40 & 1.22 & 1.33 & 0.01 & 0.01 & 0.03 & 0.06 & 0.02 & 0.01 & 1.47 & 1.74 & 1.75 & 1.32 & 0.93 & 0.82 & 0.25 & 0.18 & 0.19 & 0.13 & 0.19 & 0.10 & n.a. & n.a. & n.a. & 0.70 & 0.81 & 0.81 \\
\hline Paiements du 2ème pilier & 0.80 & 0.64 & 0.79 & 0.09 & 0.11 & 0.27 & 0.56 & 0.20 & 0.09 & 0.75 & 1.04 & 1.11 & 3.87 & 2.50 & 2.81 & 0.10 & 0.05 & 0.12 & 0.00 & 0.00 & 0.02 & n.a. & n.a. & n.a. & 0.48 & 1.44 & 0.37 \\
\hline Autres paiements & 1.96 & 1.09 & 1.04 & 0.79 & 2.03 & 4.19 & 0.35 & 0.74 & 1.02 & 0.61 & 1.23 & 0.25 & 1.65 & 0.00 & 0.04 & 0.60 & 0.13 & 0.11 & 0.88 & 0.25 & 0.60 & n.a. & n.a. & n.a. & 0.76 & 1.07 & 1.32 \\
\hline Soutien des prix du marché ${ }^{3}$ & 0.69 & 0.61 & 0.89 & 0.28 & 0.35 & 0.96 & 0.15 & 0.16 & 0.61 & 1.60 & 1.46 & 1.00 & 0.43 & 0.97 & 0.92 & 1.57 & 0.94 & -0.09 & 2.21 & 2.43 & 4.35 & n.a. & n.a. & n.a. & 0.92 & 1.04 & 1.38 \\
\hline Recettes brutes ${ }^{4}$ & 0.72 & 0.80 & 0.72 & 2.11 & 2.05 & 2.19 & 0.87 & 1.02 & 1.09 & 0.76 & 0.75 & 0.79 & 0.36 & 0.42 & 0.38 & 1.66 & 1.53 & 1.25 & 1.89 & 1.85 & 2.00 & n.a. & n.a. & n.a. & 1.10 & 1.07 & 1.08 \\
\hline Dépenses d'exploitation & 0.73 & 0.67 & 0.63 & 2.19 & 2.19 & 2.31 & 0.80 & 0.93 & 0.93 & 0.66 & 0.67 & 0.65 & 0.33 & 0.44 & 0.42 & 1.71 & 1.66 & 1.70 & 2.27 & 2.33 & 2.21 & n.a. & n.a. & n.a. & 1.24 & 1.14 & 1.15 \\
\hline Revenu net d'exploitation ${ }^{5}$ & 0.69 & 1.12 & 0.98 & 1.84 & 1.70 & 1.84 & 1.08 & 1.27 & 1.60 & 1.07 & 0.95 & 1.22 & 0.44 & 0.37 & 0.25 & 1.51 & 1.19 & -0.13 & 0.66 & 0.61 & 1.37 & n.a. & n.a. & n.a. & 0.66 & 0.88 & 0.87 \\
\hline Amortissements ${ }^{6}$ & 0.93 & 0.82 & 0.80 & 2.17 & 2.08 & 2.27 & 1.03 & 1.15 & 1.14 & 0.72 & 0.76 & 0.73 & 0.52 & 0.63 & 0.58 & 1.11 & 1.10 & 1.06 & 1.46 & 1.60 & 1.38 & n.a. & n.a. & n.a. & 0.97 & 0.95 & 0.99 \\
\hline Revenu agricole $^{7}$ & 0.45 & 1.33 & 1.12 & 1.49 & 1.44 & 1.50 & 1.13 & 1.36 & 1.96 & 1.44 & 1.08 & 1.60 & 0.37 & 0.19 & -0.01 & 1.92 & 1.25 & -1.06 & -0.17 & -0.07 & 1.37 & n.a. & n.a. & n.a. & 0.33 & 0.84 & 0.78 \\
\hline Revenu non agricole ${ }^{8}$ & 1.07 & 0.98 & 1.05 & 0.58 & 0.55 & 0.56 & 0.89 & 0.97 & 0.99 & 1.10 & 1.01 & 0.84 & 0.76 & 1.41 & 1.62 & 1.20 & 0.80 & 1.08 & 1.05 & 0.71 & 0.75 & n.a. & n.a. & n.a. & 1.18 & 1.09 & 0.93 \\
\hline Revenu des ménages agricoles ${ }^{9}$ & 0.63 & 1.24 & 1.10 & 1.23 & 1.20 & 1.24 & 1.06 & 1.26 & 1.70 & 1.34 & 1.06 & 1.40 & 0.48 & 0.51 & 0.43 & 1.72 & 1.13 & -0.49 & 0.18 & 0.14 & 1.20 & n.a. & n.a. & n.a. & 0.58 & 0.91 & 0.82 \\
\hline Total des actifs agricoles ${ }^{11}$ & 0.95 & 1.03 & 1.10 & 0.85 & 0.96 & 1.17 & 0.59 & 0.61 & 0.65 & 1.46 & 1.35 & 1.25 & 0.44 & 0.47 & 0.44 & 0.71 & 0.81 & 0.79 & 0.75 & 0.84 & 0.82 & n.a. & n.a. & n.a. & 0.90 & 0.99 & 1.02 \\
\hline Total des dettes agricoles ${ }^{12}$ & 0.85 & 0.80 & 0.82 & 1.37 & 1.39 & 1.58 & 0.63 & 0.59 & 0.66 & 1.15 & 1.14 & 1.11 & 0.35 & 0.49 & 0.44 & 1.24 & 1.17 & 1.18 & 1.36 & 1.48 & 1.21 & n.a. & n.a. & n.a. & 1.02 & 1.03 & 1.02 \\
\hline Capitaux propres agricoles ${ }^{13}$ & 1.00 & 1.15 & 1.27 & 0.58 & 0.72 & 0.92 & 0.56 & 0.62 & 0.65 & 1.62 & 1.46 & 1.34 & 0.48 & 0.46 & 0.44 & 0.43 & 0.61 & 0.56 & 0.43 & 0.49 & 0.58 & n.a. & n.a. & n.a. & 0.83 & 0.97 & 1.02 \\
\hline
\end{tabular}




\begin{tabular}{|c|c|c|c|c|c|c|c|c|c|c|c|c|c|c|c|c|c|c|c|c|c|c|c|c|c|c|c|}
\hline \multirow{3}{*}{$\begin{array}{l}\text { Pays } \\
\text { Type d'exploitation } \\
\text { Année }\end{array}$} & \multicolumn{27}{|c|}{ Finlande } \\
\hline & \multicolumn{3}{|c|}{ Grandes cultures $^{14}$} & \multicolumn{3}{|c|}{$\begin{array}{l}\text { Pépinières, serres et } \\
\text { floriculture }\end{array}$} & \multicolumn{3}{|c|}{ Fruits et légumes } & \multicolumn{3}{|c|}{ Lait } & \multicolumn{3}{|c|}{ Bovins } & \multicolumn{3}{|c|}{ Porcins } & \multicolumn{3}{|c|}{ Volaille/oeufs } & \multicolumn{3}{|c|}{ Ovins/caprins } & \multicolumn{3}{|c|}{ Autres } \\
\hline & 2004 & 2006 & 2007 & 2004 & 2006 & 2007 & 2004 & 2006 & 2007 & 2004 & 2006 & 2007 & 2004 & 2006 & 2007 & 2004 & 2006 & 2007 & 2004 & 2006 & 2007 & 2004 & 2006 & 2007 & 2004 & 2006 & 2007 \\
\hline Exploitations couvertes & 0.28 & 0.30 & 0.29 & 0.06 & 0.06 & 0.06 & n.a. & n.a. & n.a. & 0.34 & 0.31 & 0.29 & 0.05 & 0.05 & 0.06 & 0.02 & 0.02 & 0.01 & n.a. & n.a. & n.a. & n.a. & n.a. & n.a. & n.a. & n.a. & n.a. \\
\hline Unité de travail (éq. temps plein) & 0.45 & 0.45 & 0.47 & 2.05 & 2.01 & 2.33 & n.a. & n.a. & n.a. & 1.36 & 1.46 & 1.44 & 0.86 & 0.94 & 0.94 & 1.14 & 1.27 & 1.19 & n.a. & n.a. & n.a. & n.a. & n.a. & n.a. & n.a. & n.a. & n.a. \\
\hline Superficie agricole utilisée & 1.19 & 1.16 & 1.18 & 0.24 & 0.26 & 0.15 & n.a. & n.a. & n.a. & 0.89 & 0.90 & 0.91 & 0.99 & 1.10 & 1.08 & 1.04 & 1.04 & 1.07 & n.a. & n.a. & n.a. & ก.a. & n.a. & n.a. & n.a. & n.a. & n.a. \\
\hline Part en \% des exploit. couvertes & 28 & 30 & 29 & 6 & 6 & 6 & n.a. & n.a. & n.a. & 34 & 31 & 29 & 5 & 5 & 6 & 2 & 2 & 1 & n.a. & n.a. & n.a. & n.a. & n.a. & n.a. & n.a. & n.a. & n.a. \\
\hline Part en \% des unités de travail & 13 & 13 & 14 & 12 & 12 & 14 & n.a. & n.a. & n.a. & 46 & 46 & 42 & 4 & 5 & 5 & 3 & 3 & 2 & n.a. & n.a. & n.a. & n.a. & n.a. & n.a. & n.a. & n.a. & n.a. \\
\hline Part en \% de la superficie & 34 & 34 & 34 & 1 & 2 & 1 & n.a. & n.a. & n.a. & 30 & 28 & 27 & 5 & 6 & 6 & 3 & 2 & 1 & n.a. & n.a. & n.a. & n.a. & n.a. & n.a. & n.a. & n.a. & n.a. \\
\hline \multicolumn{28}{|l|}{ Composantes du revenu } \\
\hline Production agricole brute ${ }^{1}$ & 0.36 & 0.42 & 0.61 & 2.53 & 2.55 & 2.51 & n.a. & n.a. & n.a. & 1.26 & 1.30 & 1.21 & 0.66 & 0.73 & 0.66 & 2.21 & 2.34 & 2.65 & n.a. & n.a. & n.a. & n.a. & n.a. & n.a. & n.a. & n.a. & n.a. \\
\hline Soutien total & n.a. & n.a. & n.a. & n.a. & n.a. & n.a. & n.a. & n.a. & n.a. & n.a. & n.a. & n.a. & n.a. & n.a. & n.a. & n.a. & n.a. & n.a. & n.a. & n.a. & n.a. & n.a. & n.a. & n.a. & n.a. & n.a. & n.a. \\
\hline Tous paiements $^{2}$ & 0.81 & 0.78 & 0.81 & 0.65 & 0.64 & 0.65 & n.a. & n.a. & n.a. & 1.05 & 1.13 & 1.11 & 1.70 & 1.60 & 1.66 & 1.32 & 1.28 & 1.42 & n.a. & n.a. & n.a. & n.a. & n.a. & n.a. & n.a. & n.a. & n.a. \\
\hline Paiements du 1er pilier & 1.16 & 1.00 & 1.02 & 0.04 & 0.08 & 0.08 & n.a. & n.a. & n.a. & 0.77 & 1.02 & 1.01 & 2.43 & 1.73 & 1.78 & 0.99 & 0.83 & 0.79 & n.a. & n.a. & n.a. & n.a. & n.a. & n.a. & n.a. & n.a. & n.a. \\
\hline Paiements du 2ème pilier & 1.08 & 1.04 & 1.09 & 0.22 & 0.25 & 0.19 & n.a. & n.a. & n.a. & 0.92 & 0.94 & 0.89 & 1.20 & 1.34 & 1.31 & 0.99 & 1.03 & 1.10 & n.a. & n.a. & n.a. & n.a. & n.a. & n.a. & n.a. & n.a. & n.a. \\
\hline Autres paiements & 0.26 & 0.31 & 0.30 & 1.59 & 1.51 & 1.66 & n.a. & n.a. & n.a. & 1.40 & 1.42 & 1.47 & 1.72 & 1.78 & 1.97 & 1.93 & 1.93 & 2.33 & n.a. & n.a. & n.a. & n.a. & n.a. & n.a. & n.a. & n.a. & n.a. \\
\hline Soutien des prix du marché ${ }^{3}$ & n.a. & n.a. & n.a. & n.a. & n.a. & n.a. & n.a. & n.a. & n.a. & n.a. & n.a. & n.a. & n.a. & n.a. & n.a. & n.a. & n.a. & n.a. & n.a. & n.a. & n.a. & n.a. & n.a. & n.a. & n.a. & n.a. & n.a. \\
\hline Recettes brutes ${ }^{4}$ & 0.54 & 0.57 & 0.68 & 1.78 & 1.76 & 1.82 & n.a. & n.a. & n.a. & 1.17 & 1.23 & 1.18 & 1.08 & 1.09 & 1.03 & 1.85 & 1.90 & 2.20 & n.a. & n.a. & n.a. & n.a. & n.a. & n.a. & n.a. & n.a. & n.a. \\
\hline Dépenses d'exploitation & 0.53 & 0.52 & 0.59 & 2.16 & 2.16 & 2.28 & n.a. & n.a. & n.a. & 0.98 & 1.06 & 1.05 & 1.26 & 1.28 & 1.23 & 1.72 & 1.92 & 2.37 & n.a. & n.a. & n.a. & n.a. & n.a. & n.a. & n.a. & n.a. & n.a. \\
\hline Revenu net d'exploitation ${ }^{5}$ & 0.56 & 0.66 & 0.83 & 1.11 & 1.04 & 1.07 & n.a. & n.a. & n.a. & 1.51 & 1.53 & 1.38 & 0.76 & 0.74 & 0.70 & 2.08 & 1.87 & 1.91 & n.a. & n.a. & n.a. & n.a. & n.a. & n.a. & n.a. & n.a. & n.a. \\
\hline Amortissements ${ }^{6}$ & 0.77 & 0.72 & 0.77 & 0.92 & 0.83 & 1.03 & n.a. & n.a. & n.a. & 1.10 & 1.19 & 1.18 & 1.31 & 1.34 & 1.24 & 1.85 & 1.79 & 1.96 & n.a. & n.a. & n.a. & n.a. & n.a. & n.a. & n.a. & n.a. & n.a. \\
\hline Revenu agricole $^{7}$ & 0.36 & 0.59 & 0.89 & 1.29 & 1.27 & 1.11 & n.a. & n.a. & n.a. & 1.92 & 1.88 & 1.56 & 0.22 & 0.11 & 0.20 & 2.31 & 1.95 & 1.87 & n.a. & n.a. & n.a. & n.a. & n.a. & n.a. & n.a. & n.a. & n.a. \\
\hline Revenu non agricole ${ }^{8}$ & n.a. & n.a. & n.a. & n.a. & n.a. & n.a. & n.a. & n.a. & n.a. & n.a. & n.a. & n.a. & n.a. & n.a. & n.a. & n.a. & n.a. & n.a. & n.a. & n.a. & n.a. & n.a. & n.a. & n.a. & n.a. & n.a. & n.a. \\
\hline Revenu des ménages agricoles ${ }^{9}$ & n.a. & n.a. & n.a. & n.a. & n.a. & n.a. & n.a. & n.a. & n.a. & n.a. & n.a. & n.a. & n.a. & n.a. & n.a. & n.a. & n.a. & n.a. & n.a. & n.a. & n.a. & n.a. & n.a. & n.a. & n.a. & n.a. & n.a. \\
\hline Total des actifs agricoles ${ }^{11}$ & 0.87 & 0.87 & 0.90 & 0.78 & 0.70 & 0.91 & n.a. & n.a. & n.a. & 1.07 & 1.10 & 1.09 & 1.24 & 1.25 & 1.14 & 1.69 & 1.65 & 1.82 & n.a. & n.a. & n.a. & n.a. & n.a. & n.a. & n.a. & n.a. & n.a. \\
\hline Total des dettes agricoles ${ }^{12}$ & 0.68 & 0.67 & 0.63 & 1.50 & 1.42 & 1.96 & n.a. & n.a. & n.a. & 0.99 & 1.10 & 1.15 & 1.61 & 1.54 & 1.33 & 1.80 & 2.06 & 2.05 & n.a. & n.a. & n.a. & n.a. & n.a. & n.a. & n.a. & n.a. & n.a. \\
\hline Capitaux propres agricoles ${ }^{13}$ & 0.93 & 0.94 & 0.99 & 0.54 & 0.45 & 0.53 & n.a. & n.a. & n.a. & 1.09 & 1.10 & 1.07 & 1.12 & 1.15 & 1.07 & 1.65 & 1.51 & 1.74 & n.a. & n.a. & n.a. & n.a. & n.a. & n.a. & n.a. & n.a. & n.a. \\
\hline
\end{tabular}


90 - Distribution DU SOUtien ET DES REVENUS DANS L'AGRICULtURE

\begin{tabular}{|c|c|c|c|c|c|c|c|c|c|c|c|c|c|c|c|c|c|c|c|c|c|c|c|c|c|c|c|}
\hline \multirow{3}{*}{$\begin{array}{l}\text { Pays } \\
\text { Type d'exploitation } \\
\text { Année }\end{array}$} & \multicolumn{27}{|c|}{ France } \\
\hline & \multicolumn{3}{|c|}{ Grandes cultures $^{14}$} & \multicolumn{3}{|c|}{$\begin{array}{l}\text { Pépinières, serres et } \\
\text { floriculture }\end{array}$} & \multicolumn{3}{|c|}{ Fruits et légumes } & \multicolumn{3}{|c|}{ Lait } & \multicolumn{3}{|c|}{ Bovins } & \multicolumn{3}{|c|}{ Porcins } & \multicolumn{3}{|c|}{ Volaille/oeufs } & \multicolumn{3}{|c|}{ Ovins/caprins } & \multicolumn{3}{|c|}{ Autres } \\
\hline & 2004 & 2006 & 2007 & 2004 & 2006 & 2007 & 2004 & 2006 & 2007 & 2004 & 2006 & 2007 & 2004 & 2006 & 2007 & 2004 & 2006 & 2007 & 2004 & 2006 & 2007 & 2004 & 2006 & 2007 & 2004 & 2006 & 2007 \\
\hline Exploitations couvertes & 0.22 & 0.22 & 0.23 & 0.02 & 0.02 & 0.01 & 0.18 & 0.18 & 0.18 & 0.17 & 0.17 & 0.16 & 0.14 & 0.14 & 0.15 & 0.01 & 0.01 & 0.01 & 0.01 & 0.01 & 0.01 & 0.04 & 0.04 & 0.05 & 0.21 & 0.21 & 0.20 \\
\hline Unité de travail (éq. temps plein) & 0.83 & 0.83 & 0.83 & 2.17 & 2.09 & 2.16 & 1.61 & 1.60 & 1.59 & 0.85 & 0.86 & 0.86 & 0.71 & 0.71 & 0.71 & 0.98 & 1.02 & 1.14 & 0.85 & 0.82 & 0.83 & 0.76 & 0.74 & 0.75 & 0.95 & 0.96 & 0.96 \\
\hline Superficie agricole utilisée & 1.48 & 1.48 & 1.45 & 0.05 & 0.04 & 0.03 & 0.31 & 0.30 & 0.29 & 0.92 & 0.92 & 0.92 & 1.18 & 1.18 & 1.19 & 0.44 & 0.47 & 0.46 & 0.24 & 0.21 & 0.27 & 0.94 & 1.01 & 1.01 & 1.16 & 1.16 & 1.17 \\
\hline Part en \% des exploit. couvertes & 22 & 22 & 23 & 2 & 2 & 1 & 18 & 18 & 18 & 17 & 17 & 16 & 14 & 14 & 15 & 1 & 1 & 1 & 1 & 1 & 1 & 4 & 4 & 5 & 21 & 21 & 20 \\
\hline Part en \%des unités de travail & 19 & 19 & 19 & 3 & 3 & 3 & 29 & 29 & 28 & 14 & 15 & 14 & 10 & 10 & 10 & 1 & 1 & 1 & 1 & 1 & 1 & 3 & 3 & 3 & 20 & 20 & 20 \\
\hline Part en \% de la superficie & 33 & 33 & 33 & 0 & 0 & 0 & 6 & 5 & 5 & 16 & 15 & 15 & 17 & 17 & 18 & 1 & 0 & 0 & 0 & 0 & 0 & 4 & 4 & 5 & 24 & 24 & 24 \\
\hline \multicolumn{28}{|l|}{ Composantes du revenu } \\
\hline Production agricole brute ${ }^{1}$ & 0.93 & 0.97 & 1.08 & 1.70 & 1.78 & 1.61 & 1.41 & 1.35 & 1.28 & 0.87 & 0.84 & 0.85 & 0.57 & 0.59 & 0.54 & 2.46 & 3.04 & 2.91 & 1.25 & 1.24 & 1.36 & 0.51 & 0.48 & 0.44 & 1.07 & 1.08 & 1.10 \\
\hline Soutien total & n.a. & n.a. & n.a. & n.a. & n.a. & n.a. & n.a. & n.a. & n.a. & n.a. & n.a. & n.a. & n.a. & n.a. & n.a. & n.a. & n.a. & n.a. & n.a. & n.a. & n.a. & n.a. & n.a. & n.a. & n.a. & n.a. & n.a. \\
\hline Tous paiements ${ }^{2}$ & 1.50 & 1.44 & 1.47 & 0.10 & 0.08 & 0.09 & 0.26 & 0.23 & 0.23 & 0.75 & 0.88 & 0.89 & 1.36 & 1.35 & 1.27 & 0.49 & 0.50 & 0.47 & 0.31 & 0.26 & 0.38 & 0.98 & 0.90 & 0.87 & 1.18 & 1.20 & 1.18 \\
\hline Paiements du 1er pilier & 1.76 & 1.64 & 1.64 & 0.03 & 0.01 & 0.03 & 0.16 & 0.16 & 0.15 & 0.66 & 0.84 & 0.85 & 1.23 & 1.22 & 1.17 & 0.50 & 0.46 & 0.47 & 0.32 & 0.26 & 0.35 & 0.57 & 0.51 & 0.52 & 1.23 & 1.24 & 1.26 \\
\hline Paiements du zème pilier & 0.37 & 0.36 & 0.21 & 0.04 & 0.02 & 0.02 & 0.19 & 0.16 & 0.10 & 1.37 & 1.40 & 1.49 & 2.31 & 2.35 & 2.49 & 0.29 & 0.30 & 0.30 & 0.10 & 0.07 & 0.12 & 3.40 & 3.29 & 3.64 & 0.86 & 0.85 & 0.74 \\
\hline Autres paiements & 0.84 & 0.89 & 1.26 & 0.63 & 0.73 & 0.74 & 1.03 & 0.92 & 1.09 & 0.80 & 0.65 & 0.66 & 1.32 & 1.36 & 0.94 & 0.63 & 1.01 & 0.68 & 0.37 & 0.44 & 0.92 & 1.36 & 1.60 & 1.24 & 1.10 & 1.15 & 0.93 \\
\hline Soutien des prix du marché ${ }^{3}$ & n.a. & n.a. & n.a. & n.a. & n.a. & n.a. & n.a. & n.a. & n.a. & n.a. & n.a. & n.a. & n.a. & n.a. & n.a. & n.a. & n.a. & n.a. & n.a. & n.a. & n.a. & n.a. & n.a. & n.a. & n.a. & n.a. & n.a. \\
\hline Recettes brutes ${ }^{4}$ & 1.03 & 1.05 & 1.14 & 1.42 & 1.48 & 1.37 & 1.21 & 1.15 & 1.11 & 0.85 & 0.85 & 0.86 & 0.70 & 0.73 & 0.66 & 2.13 & 2.59 & 2.53 & 1.09 & 1.06 & 1.21 & 0.59 & 0.56 & 0.51 & 1.09 & 1.10 & 1.11 \\
\hline Dépenses d'exploitation & 1.03 & 1.05 & 1.06 & 1.66 & 1.79 & 1.71 & 1.20 & 1.18 & 1.14 & 0.80 & 0.81 & 0.83 & 0.65 & 0.66 & 0.64 & 2.69 & 2.96 & 3.52 & 1.22 & 1.20 & 1.43 & 0.57 & 0.53 & 0.50 & 1.12 & 1.12 & 1.14 \\
\hline Revenu net d'exploitation ${ }^{5}$ & 1.01 & 1.06 & 1.31 & 0.94 & 0.85 & 0.72 & 1.23 & 1.09 & 1.06 & 0.95 & 0.92 & 0.92 & 0.82 & 0.87 & 0.68 & 0.96 & 1.85 & 0.68 & 0.82 & 0.79 & 0.80 & 0.64 & 0.62 & 0.53 & 1.04 & 1.07 & 1.05 \\
\hline Amortissements $^{6}$ & 1.08 & 1.06 & 1.04 & 1.18 & 1.03 & 1.01 & 0.98 & 0.91 & 0.88 & 0.95 & 0.99 & 1.04 & 0.79 & 0.84 & 0.87 & 1.42 & 1.67 & 1.99 & 0.93 & 0.83 & 0.91 & 0.69 & 0.69 & 0.67 & 1.13 & 1.17 & 1.16 \\
\hline Revenu agricole $^{7}$ & 0.96 & 1.06 & 1.48 & 0.75 & 0.72 & 0.54 & 1.41 & 1.23 & 1.17 & 0.95 & 0.87 & 0.85 & 0.85 & 0.89 & 0.56 & 0.62 & 1.97 & -0.13 & 0.74 & 0.76 & 0.72 & 0.59 & 0.57 & 0.44 & 0.96 & 0.99 & 0.98 \\
\hline Revenu non agricole ${ }^{8}$ & n.a. & n.a. & n.a. & n.a. & n.a. & n.a. & n.a. & n.a. & n.a. & n.a. & n.a. & n.a. & n.a. & n.a. & n.a. & n.a. & n.a. & n.a. & n.a. & n.a. & n.a. & n.a. & n.a. & n.a. & n.a. & n.a. & n.a. \\
\hline Revenu des ménages agricoles ${ }^{9}$ & n.a. & n.a. & n.a. & n.a. & n.a. & n.a. & n.a. & n.a. & n.a. & n.a. & n.a. & n.a. & n.a. & n.a. & n.a. & n.a. & n.a. & n.a. & n.a. & n.a. & n.a. & n.a. & n.a. & n.a. & n.a. & n.a. & n.a. \\
\hline Total des actifs agricoles ${ }^{11}$ & n.a. & n.a. & n.a. & n.a. & n.a. & n.a. & n.a. & n.a. & n.a. & n.a. & n.a. & n.a. & n.a. & n.a. & n.a. & n.a. & n.a. & n.a. & n.a. & n.a. & n.a. & n.a. & n.a. & n.a. & n.a. & n.a. & n.a. \\
\hline Total des dettes agricoles ${ }^{12}$ & n.a. & n.a. & n.a. & n.a. & n.a. & n.a. & n.a. & n.a. & n.a. & n.a. & n.a. & n.a. & n.a. & n.a. & n.a. & n.a. & n.a. & n.a. & n.a. & n.a. & n.a. & n.a. & n.a. & n.a. & n.a. & n.a. & n.a. \\
\hline Capitaux propres agricoles ${ }^{13}$ & n.a. & n.a. & n.a. & n.a. & n.a. & n.a. & n.a. & n.a. & n.a. & n.a. & n.a. & n.a. & n.a. & n.a. & n.a. & n.a. & n.a. & n.a. & n.a. & n.a. & n.a. & n.a. & n.a. & n.a. & n.a. & n.a. & n.a. \\
\hline
\end{tabular}




\begin{tabular}{|c|c|c|c|c|c|c|c|c|c|c|c|c|c|c|c|c|c|c|c|c|c|c|c|c|c|c|c|}
\hline \multirow{3}{*}{$\begin{array}{l}\text { Pays } \\
\text { Type d'exploitation } \\
\text { Année }\end{array}$} & \multicolumn{27}{|c|}{ Allemagne } \\
\hline & \multicolumn{3}{|c|}{ Grandes cultures $^{14}$} & \multicolumn{3}{|c|}{$\begin{array}{l}\text { Pépinières, serres et } \\
\text { floriculture }\end{array}$} & \multicolumn{3}{|c|}{ Fruits et légumes } & \multicolumn{3}{|c|}{ Lait } & \multicolumn{3}{|c|}{ Bovins } & \multicolumn{3}{|c|}{ Porcins } & \multicolumn{3}{|c|}{ Volaille/oeufs } & \multicolumn{3}{|c|}{ Ovins/caprins } & \multicolumn{3}{|c|}{ Autres } \\
\hline & 2004 & 2006 & 2007 & 2004 & 2006 & 2007 & 2004 & 2006 & 2007 & 2004 & 2006 & 2007 & 2004 & 2006 & 2007 & 2004 & 2006 & 2007 & 2004 & 2006 & 2007 & 2004 & 2006 & 2007 & 2004 & 2006 & 2007 \\
\hline Exploitations couvertes & 0.28 & 0.23 & 0.23 & 0.03 & 0.04 & 0.05 & 0.07 & 0.07 & 0.07 & 0.25 & 0.31 & 0.31 & 0.06 & 0.07 & 0.07 & 0.01 & 0.03 & 0.03 & 0.00 & 0.00 & 0.00 & 0.00 & 0.00 & 0.00 & 0.29 & 0.24 & 0.23 \\
\hline Unité de travail (éq. temps plein) & 0.89 & 0.85 & 0.83 & 2.64 & 2.33 & 2.45 & 1.26 & 1.19 & 1.18 & 0.82 & 0.86 & 0.85 & 0.84 & 0.78 & 0.83 & 0.96 & 0.92 & 0.93 & 1.09 & 1.33 & 1.15 & 0.92 & 0.75 & 0.83 & 1.04 & 1.10 & 1.09 \\
\hline Superficie agricole utilisée & 1.49 & 1.51 & 1.49 & 0.07 & 0.06 & 0.07 & 0.15 & 0.14 & 0.14 & 0.66 & 0.77 & 0.78 & 0.94 & 0.91 & 0.96 & 0.69 & 0.70 & 0.72 & 0.69 & 1.14 & 0.91 & 1.98 & 1.59 & 1.39 & 1.14 & 1.30 & 1.30 \\
\hline Part en \% des exploit. couvertes & 28 & 23 & 23 & 3 & 4 & 5 & 7 & 7 & 7 & 25 & 31 & 31 & 6 & 7 & 7 & 1 & 3 & 3 & 0 & 0 & 0 & 0 & 0 & 0 & 29 & 24 & 23 \\
\hline Part en \% des unités de travail & 25 & 20 & 19 & 9 & 10 & 11 & 9 & 8 & 8 & 21 & 26 & 26 & 5 & 6 & 6 & 1 & 3 & 3 & 0 & 0 & 0 & 0 & 0 & 0 & 31 & 26 & 26 \\
\hline Part en \% de la superficie & 41 & 35 & 34 & 0 & 0 & 0 & 1 & 1 & 1 & 17 & 24 & 24 & 5 & 7 & 7 & 1 & 2 & 2 & 0 & 0 & 0 & 1 & 1 & 1 & 34 & 30 & 31 \\
\hline \multicolumn{28}{|l|}{ Composantes du revenu } \\
\hline Production agricole brute ${ }^{1}$ & 0.95 & 0.85 & 0.87 & 2.31 & 1.88 & 1.83 & 0.91 & 0.78 & 0.75 & 0.71 & 0.79 & 0.78 & 0.68 & 0.66 & 0.68 & 1.87 & 1.83 & 1.88 & 2.82 & 2.68 & 2.73 & 0.32 & 0.26 & 0.27 & 1.19 & 1.30 & 1.29 \\
\hline Soutien total & 1.10 & 1.04 & 1.11 & 0.18 & 0.19 & 0.21 & 0.11 & 0.10 & 0.11 & 1.00 & 1.13 & 1.08 & 1.11 & 1.12 & 1.23 & 0.91 & 0.65 & 0.52 & 1.90 & 1.93 & 1.95 & 1.16 & 1.22 & 1.32 & 1.17 & 1.21 & 1.19 \\
\hline Tous paiements ${ }^{2}$ & 1.42 & 1.31 & 1.28 & 0.11 & 0.09 & 0.09 & 0.13 & 0.09 & 0.09 & 0.58 & 0.90 & 0.95 & 1.27 & 1.13 & 1.17 & 0.72 & 0.60 & 0.57 & 0.68 & 1.06 & 0.86 & 2.01 & 1.82 & 1.62 & 1.21 & 1.28 & 1.25 \\
\hline Paiements du 1er pilier & 1.60 & 1.45 & 1.40 & 0.01 & 0.05 & 0.05 & 0.04 & 0.04 & 0.04 & 0.42 & 0.80 & 0.87 & 1.11 & 1.03 & 1.05 & 0.74 & 0.62 & 0.59 & 0.71 & 1.12 & 0.82 & 0.99 & 1.12 & 1.12 & 1.26 & 1.33 & 1.30 \\
\hline Paiements du Zème pilier & 0.76 & 0.80 & 0.79 & 0.11 & 0.04 & 0.07 & 0.27 & 0.19 & 0.22 & 1.20 & 1.28 & 1.30 & 2.15 & 1.66 & 1.89 & 0.38 & 0.41 & 0.37 & 0.41 & 0.55 & 0.84 & 7.03 & 5.29 & 4.46 & 1.05 & 1.07 & 1.00 \\
\hline Autres paiements & 1.24 & 0.97 & 0.94 & 0.97 & 0.78 & 0.85 & 0.55 & 0.38 & 0.45 & 0.71 & 1.06 & 1.07 & 0.90 & 0.86 & 0.81 & 1.27 & 1.04 & 1.05 & 0.89 & 1.76 & 1.45 & 0.86 & 0.67 & 0.57 & 1.14 & 1.21 & 1.21 \\
\hline Soutien des prix du marché ${ }^{3}$ & 0.77 & 0.64 & 0.72 & 0.26 & 0.33 & 0.48 & 0.10 & 0.12 & 0.14 & 1.43 & 1.48 & 1.38 & 0.95 & 1.09 & 1.37 & 1.10 & 0.71 & 0.42 & 3.15 & 3.19 & 4.37 & 0.28 & 0.34 & 0.65 & 1.14 & 1.11 & 1.07 \\
\hline Recettes brutes ${ }^{4}$ & 1.02 & 0.91 & 0.93 & 2.00 & 1.63 & 1.58 & 0.80 & 0.68 & 0.66 & 0.69 & 0.81 & 0.81 & 0.76 & 0.72 & 0.75 & 1.71 & 1.66 & 1.70 & 2.52 & 2.45 & 2.46 & 0.56 & 0.48 & 0.46 & 1.19 & 1.30 & 1.28 \\
\hline Dépenses d'exploitation & 0.99 & 0.93 & 0.92 & 2.16 & 1.76 & 1.75 & 0.70 & 0.61 & 0.59 & 0.62 & 0.70 & 0.70 & 0.79 & 0.74 & 0.77 & 1.90 & 1.73 & 1.88 & 2.83 & 2.81 & 2.70 & 0.45 & 0.37 & 0.40 & 1.27 & 1.39 & 1.38 \\
\hline Revenu net d'exploitation ${ }^{5}$ & 1.13 & 0.85 & 0.97 & 1.50 & 1.27 & 1.12 & 1.11 & 0.88 & 0.85 & 0.90 & 1.12 & 1.09 & 0.68 & 0.69 & 0.69 & 1.11 & 1.43 & 1.19 & 1.54 & 1.43 & 1.81 & 0.89 & 0.78 & 0.63 & 0.94 & 1.01 & 1.00 \\
\hline Amortissements ${ }^{6}$ & 1.00 & 0.92 & 0.92 & 1.36 & 1.05 & 0.99 & 0.75 & 0.67 & 0.63 & 0.91 & 1.08 & 1.09 & 0.83 & 0.73 & 0.75 & 1.33 & 1.16 & 1.19 & 1.51 & 1.66 & 1.56 & 0.53 & 0.53 & 0.50 & 1.11 & 1.12 & 1.13 \\
\hline Revenu agricole $^{7}$ & 1.23 & 0.80 & 1.00 & 1.60 & 1.41 & 1.19 & 1.39 & 1.01 & 0.98 & 0.90 & 1.14 & 1.09 & 0.55 & 0.67 & 0.66 & 0.94 & 1.61 & 1.19 & 1.57 & 1.28 & 1.95 & 1.18 & 0.94 & 0.71 & 0.79 & 0.94 & 0.92 \\
\hline Revenu non agricole ${ }^{8}$ & n.a. & n.a. & n.a. & n.a. & n.a. & n.a. & n.a. & n.a. & n.a. & n.a. & n.a. & n.a. & n.a. & n.a. & n.a. & n.a. & n.a. & n.a. & n.a. & n.a. & n.a. & n.a. & n.a. & n.a. & n.a. & n.a. & n.a. \\
\hline Revenu des ménages agricoles ${ }^{9}$ & n.a. & n.a. & n.a. & n.a. & n.a. & n.a. & n.a. & n.a. & n.a. & n.a. & n.a. & n.a. & n.a. & n.a. & n.a. & n.a. & n.a. & n.a. & n.a. & n.a. & n.a. & n.a. & n.a. & n.a. & n.a. & n.a. & n.a. \\
\hline Total des actifs agricoles ${ }^{11}$ & 1.19 & 1.16 & 1.17 & 0.59 & 0.51 & 0.52 & 0.67 & 0.64 & 0.62 & 0.90 & 0.98 & 0.98 & 0.93 & 0.95 & 0.91 & 0.99 & 1.04 & 1.07 & 1.24 & 1.40 & 1.22 & 0.36 & 0.38 & 0.37 & 1.05 & 1.08 & 1.10 \\
\hline Total des dettes agricoles ${ }^{12}$ & 1.00 & 0.99 & 1.03 & 2.00 & 1.54 & 1.52 & 0.84 & 0.70 & 0.63 & 0.79 & 0.89 & 0.89 & 0.86 & 0.96 & 0.81 & 1.67 & 1.42 & 1.51 & 2.21 & 2.26 & 1.93 & 0.70 & 0.49 & 0.58 & 1.09 & 1.09 & 1.11 \\
\hline Capitaux propres agricoles ${ }^{13}$ & 1.22 & 1.20 & 1.20 & 0.32 & 0.31 & 0.31 & 0.64 & 0.62 & 0.62 & 0.93 & 1.00 & 0.99 & 0.95 & 0.95 & 0.93 & 0.85 & 0.96 & 0.98 & 1.05 & 1.23 & 1.07 & 0.29 & 0.36 & 0.33 & 1.04 & 1.07 & 1.10 \\
\hline
\end{tabular}


92 - Distribution du SOUtien Et DES REVEnUS DANS L'AGRiculture

\begin{tabular}{|c|c|c|c|c|c|c|c|c|c|c|c|c|c|c|c|c|c|c|c|c|c|c|c|c|c|c|c|}
\hline \multirow{3}{*}{$\begin{array}{l}\text { Pays } \\
\text { Type d'exploitation } \\
\text { Année }\end{array}$} & \multicolumn{27}{|c|}{ Irlande } \\
\hline & \multicolumn{3}{|c|}{ Grandes cultures $^{14}$} & \multicolumn{3}{|c|}{$\begin{array}{l}\text { Pépinières, serres et } \\
\text { floriculture }\end{array}$} & \multicolumn{3}{|c|}{ Fruits et légumes } & \multicolumn{3}{|c|}{ Lait } & \multicolumn{3}{|c|}{ Bovins } & \multicolumn{3}{|c|}{ Porcins } & \multicolumn{3}{|c|}{ Volaille/oeufs } & \multicolumn{3}{|c|}{ Ovins/caprins } & \multicolumn{3}{|c|}{ Autres } \\
\hline & 2004 & 2006 & 2007 & 2004 & 2006 & 2007 & 2004 & 2006 & 2007 & 2004 & 2006 & 2007 & 2004 & 2006 & 2007 & 2004 & 2006 & 2007 & 2004 & 2006 & 2007 & 2004 & 2006 & 2007 & 2004 & 2006 & 2007 \\
\hline Exploitations couvertes & 0.06 & 0.07 & 0.07 & n.a. & n.a. & n.a. & n.a. & n.a. & n.a. & 0.24 & 0.20 & 0.20 & 0.51 & 0.52 & 0.51 & n.a. & n.a. & n.a. & n.a. & n.a. & n.a. & 0.16 & 0.18 & 0.19 & n.a. & n.a. & n.a. \\
\hline Unité de travail (éq. temps plein) & n.a. & n.a. & n.a. & n.a. & n.a. & n.a. & n.a. & n.a. & n.a. & n.a. & n.a. & n.a. & n.a. & n.a. & n.a. & n.a. & n.a. & n.a. & n.a. & n.a. & n.a. & n.a. & n.a. & n.a. & n.a. & n.a. & n.a. \\
\hline Superficie agricole utilisée & 1.61 & 1.59 & 1.54 & n.a. & n.a. & n.a. & n.a. & n.a. & n.a. & 1.24 & 1.32 & 1.32 & 0.78 & 0.81 & 0.81 & n.a. & n.a. & n.a. & n.a. & n.a. & n.a. & 1.05 & 0.95 & 0.95 & n.a. & n.a. & n.a. \\
\hline Part en \% des exploit. couvertes & 6 & 7 & 7 & n.a. & n.a. & n.a. & n.a. & n.a. & n.a. & 24 & 20 & 20 & 51 & 52 & 51 & n.a. & n.a. & n.a. & n.a. & n.a. & n.a. & 16 & 18 & 19 & n.a. & n.a. & n.a. \\
\hline Part en \% des unités de travail & n.a. & n.a. & n.a. & n.a. & n.a. & n.a. & n.a. & n.a. & n.a. & n.a. & n.a. & n.a. & n.a. & n.a. & n.a. & n.a. & n.a. & n.a. & n.a. & n.a. & n.a. & n.a. & n.a. & n.a. & n.a. & n.a. & n.a. \\
\hline Part en \%de la superficie & 10 & 11 & 10 & n.a. & n.a. & n.a. & n.a. & n.a. & n.a. & 30 & 27 & 27 & 40 & 42 & 42 & n.a. & n.a. & n.a. & n.a. & n.a. & n.a. & 17 & 17 & 18 & n.a. & n.a. & n.a. \\
\hline \multicolumn{28}{|l|}{ Composantes du revenu } \\
\hline Production agricole brute ${ }^{1}$ & 1.78 & 1.78 & 1.97 & n.a. & n.a. & n.a. & n.a. & n.a. & n.a. & 2.47 & 2.63 & 2.80 & 0.37 & 0.46 & 0.40 & n.a. & n.a. & n.a. & n.a. & n.a. & n.a. & 0.48 & 0.47 & 0.38 & n.a. & n.a. & n.a. \\
\hline Soutien total & 1.31 & 1.29 & 1.44 & n.a. & n.a. & n.a. & n.a. & n.a. & n.a. & 2.18 & 1.76 & 1.22 & 0.89 & 1.02 & 0.99 & n.a. & n.a. & n.a. & n.a. & n.a. & n.a. & 1.90 & 1.90 & 1.93 & n.a. & n.a. & n.a. \\
\hline Tous paiements $^{2}$ & 1.62 & 1.48 & 1.49 & n.a. & n.a. & n.a. & n.a. & n.a. & n.a. & 0.94 & 1.22 & 1.24 & 0.90 & 0.85 & 0.86 & n.a. & n.a. & n.a. & n.a. & n.a. & n.a. & 2.80 & 2.40 & 2.36 & n.a. & n.a. & n.a. \\
\hline Paiements du 1er pilier & 2.05 & 1.80 & 1.84 & n.a. & n.a. & n.a. & n.a. & n.a. & n.a. & 0.98 & 1.38 & 1.41 & 0.87 & 0.81 & 0.82 & n.a. & n.a. & n.a. & n.a. & n.a. & n.a. & 0.98 & 0.82 & 0.76 & n.a. & n.a. & n.a. \\
\hline Paiements du Zème pilier & 0.59 & 0.77 & 0.73 & n.a. & n.a. & n.a. & n.a. & n.a. & n.a. & 0.85 & 0.86 & 0.88 & 0.97 & 0.95 & 0.95 & n.a. & n.a. & n.a. & n.a. & n.a. & n.a. & 1.43 & 1.35 & 1.31 & n.a. & n.a. & n.a. \\
\hline Autres paiements & n.a. & n.a. & n.a. & n.a. & n.a. & n.a. & n.a. & n.a. & n.a. & n.a. & n.a. & n.a. & n.a. & n.a. & n.a. & n.a. & n.a. & n.a. & n.a. & n.a. & n.a. & n.a. & n.a. & n.a. & n.a. & n.a. & n.a. \\
\hline Soutien des prix du marché ${ }^{3}$ & 0.66 & 0.55 & 1.18 & n.a. & n.a. & n.a. & n.a. & n.a. & n.a. & 4.81 & 3.85 & 1.12 & 0.86 & 1.65 & 1.57 & n.a. & n.a. & n.a. & n.a. & n.a. & n.a. & 0.46 & 0.91 & 0.95 & n.a. & n.a. & n.a. \\
\hline Recettes brutes ${ }^{4}$ & 1.74 & 1.68 & 1.82 & n.a. & n.a. & n.a. & n.a. & n.a. & n.a. & 2.03 & 2.16 & 2.32 & 0.52 & 0.59 & 0.54 & n.a. & n.a. & n.a. & n.a. & n.a. & n.a. & 1.63 & 1.66 & 1.50 & n.a. & n.a. & n.a. \\
\hline Dépenses d'exploitation & 1.89 & 1.73 & 1.72 & n.a. & n.a. & n.a. & n.a. & n.a. & n.a. & 2.02 & 2.23 & 2.28 & 0.52 & 0.57 & 0.57 & n.a. & n.a. & n.a. & n.a. & n.a. & n.a. & 0.64 & 0.60 & 0.55 & n.a. & n.a. & n.a. \\
\hline Revenu net d'exploitation ${ }^{5}$ & 1.54 & 1.63 & 1.94 & n.a. & n.a. & n.a. & n.a. & n.a. & n.a. & 2.04 & 2.08 & 2.36 & 0.53 & 0.61 & 0.51 & n.a. & n.a. & n.a. & n.a. & n.a. & n.a. & 1.22 & 1.29 & 1.02 & n.a. & n.a. & n.a. \\
\hline Amortissements ${ }^{6}$ & 1.64 & 1.37 & 1.49 & n.a. & n.a. & n.a. & n.a. & n.a. & n.a. & 1.91 & 2.06 & 2.12 & 0.60 & 0.67 & 0.66 & n.a. & n.a. & n.a. & n.a. & n.a. & n.a. & 0.63 & 0.62 & 0.56 & n.a. & n.a. & n.a. \\
\hline Revenu agricole ${ }^{7}$ & 1.51 & 1.71 & 2.06 & n.a. & n.a. & n.a. & n.a. & n.a. & n.a. & 2.08 & 2.08 & 2.43 & 0.51 & 0.59 & 0.47 & n.a. & n.a. & n.a. & n.a. & n.a. & n.a. & 1.40 & 1.49 & 1.15 & n.a. & n.a. & n.a. \\
\hline Revenu non agricole ${ }^{8}$ & n.a. & n.a. & n.a. & n.a. & n.a. & n.a. & n.a. & n.a. & n.a. & n.a. & n.a. & n.a. & n.a. & n.a. & n.a. & n.a. & n.a. & n.a. & n.a. & n.a. & n.a. & n.a. & n.a. & n.a. & n.a. & n.a. & n.a. \\
\hline Revenu des ménages agricoles ${ }^{9}$ & n.a. & n.a. & n.a. & n.a. & n.a. & n.a. & n.a. & n.a. & n.a. & n.a. & n.a. & n.a. & n.a. & n.a. & n.a. & n.a. & n.a. & n.a. & n.a. & n.a. & n.a. & n.a. & n.a. & n.a. & n.a. & n.a. & n.a. \\
\hline Total des actifs agricoles ${ }^{11}$ & n.a. & n.a. & n.a. & n.a. & n.a. & n.a. & n.a. & n.a. & n.a. & n.a. & n.a. & n.a. & n.a. & n.a. & n.a. & n.a. & n.a. & n.a. & п.a. & n.a. & n.a. & n.a. & n.a. & n.a. & n.a. & n.a. & n.a. \\
\hline Total des dettes agricoles ${ }^{12}$ & n.a. & n.a. & n.a. & n.a. & n.a. & n.a. & n.a. & n.a. & n.a. & n.a. & n.a. & n.a. & n.a. & n.a. & n.a. & n.a. & n.a. & n.a. & n.a. & n.a. & n.a. & n.a. & n.a. & n.a. & n.a. & n.a. & n.a. \\
\hline Capitaux propres agricoles ${ }^{13}$ & n.a. & n.a. & n.a. & n.a. & n.a. & n.a. & n.a. & n.a. & n.a. & n.a. & n.a. & n.a. & п.а. & n.a. & n.a. & n.a. & n.a. & n.a. & n.a. & n.a. & n.a. & n.a. & n.a. & n.a. & n.a. & n.a. & n.a. \\
\hline
\end{tabular}




\begin{tabular}{|c|c|c|c|c|c|c|c|c|c|c|c|c|c|c|c|c|c|c|c|c|c|c|c|c|c|c|c|}
\hline \multirow{3}{*}{$\begin{array}{l}\text { Pays } \\
\text { Type d'exploitation } \\
\text { Année }\end{array}$} & \multicolumn{27}{|c|}{ Italie } \\
\hline & \multicolumn{3}{|c|}{ Grandes cultures $^{14}$} & \multicolumn{3}{|c|}{$\begin{array}{l}\text { Pépinières, serres et } \\
\text { floriculture }\end{array}$} & \multicolumn{3}{|c|}{ Fruits et légumes } & \multicolumn{3}{|c|}{ Lait } & \multicolumn{3}{|c|}{ Bovins } & \multicolumn{3}{|c|}{ Porcins } & \multicolumn{3}{|c|}{ Volaille/oeufs } & \multicolumn{3}{|c|}{ Ovins/caprins } & \multicolumn{3}{|c|}{ Autres } \\
\hline & 2004 & 2006 & 2007 & 2004 & 2006 & 2007 & 2004 & 2006 & 2007 & 2004 & 2006 & 2007 & 2004 & 2006 & 2007 & 2004 & 2006 & 2007 & 2004 & 2006 & 2007 & 2004 & 2006 & 2007 & 2004 & 2006 & 2007 \\
\hline Exploitations couvertes & 0.25 & 0.20 & 0.19 & 0.03 & 0.04 & 0.04 & 0.14 & 0.15 & 0.16 & 0.07 & 0.06 & 0.06 & 0.01 & 0.02 & 0.01 & 0.00 & 0.01 & 0.00 & 0.00 & 0.00 & 0.00 & 0.02 & 0.02 & 0.03 & 0.47 & 0.51 & 0.50 \\
\hline Unité de travail (éq. temps plein) & 0.88 & 0.88 & 0.88 & 1.25 & 1.31 & 1.26 & 0.95 & 1.00 & 1.03 & 1.52 & 1.53 & 1.52 & 1.19 & 1.13 & 1.12 & 2.01 & 1.92 & 2.42 & 1.45 & 1.54 & 2.21 & 1.13 & 1.07 & 1.07 & 0.97 & 0.94 & 0.93 \\
\hline Superficie agricole utilisée & 1.38 & 1.36 & 1.40 & 0.16 & 0.14 & 0.13 & 0.44 & 0.42 & 0.46 & 1.76 & 1.94 & 2.10 & 2.33 & 2.56 & 2.44 & 1.84 & 1.82 & 2.59 & 0.57 & 0.77 & 0.97 & 2.96 & 3.03 & 2.66 & 0.80 & 0.85 & 0.82 \\
\hline Part en \% des exploit. couvertes & 25 & 20 & 19 & 3 & 4 & 4 & 14 & 15 & 16 & 7 & 6 & 6 & 1 & 2 & 1 & 0 & 1 & 0 & 0 & 0 & 0 & 2 & 2 & 3 & 47 & 51 & 50 \\
\hline Part en \% des unités de travail & 22 & 17 & 17 & 4 & 5 & 5 & 14 & 15 & 17 & 10 & 9 & 9 & 1 & 2 & 1 & 1 & 1 & 1 & 1 & 0 & 0 & 2 & 2 & 3 & 46 & 48 & 46 \\
\hline Part en \% de la superficie & 34 & 27 & 27 & 0 & 1 & 1 & 6 & 6 & 8 & 12 & 11 & 12 & 3 & 5 & 3 & 1 & 1 & 1 & 0 & 0 & 0 & 6 & 6 & 7 & 38 & 43 & 41 \\
\hline \multicolumn{28}{|l|}{ Composantes du revenu } \\
\hline Production agricole brute ${ }^{1}$ & 0.50 & 0.50 & 0.62 & 2.16 & 1.66 & 1.46 & 0.81 & 0.89 & 0.96 & 3.11 & 3.17 & 3.05 & 2.05 & 2.09 & 2.11 & 12.44 & 9.96 & 13.27 & 6.66 & 6.42 & 8.96 & 0.92 & 0.98 & 0.99 & 0.80 & 0.75 & 0.73 \\
\hline Soutien total & 1.47 & 1.34 & 1.60 & 0.53 & 0.29 & 0.64 & 0.50 & 0.40 & 0.56 & 1.63 & 3.28 & 2.15 & 2.69 & 2.82 & 2.39 & 2.65 & 2.82 & 4.05 & 0.43 & 0.91 & 1.22 & 1.05 & 1.71 & 1.22 & 0.79 & 0.73 & 0.74 \\
\hline Tous paiements ${ }^{2}$ & 1.54 & 1.37 & 1.55 & 0.05 & 0.07 & 0.04 & 0.22 & 0.23 & 0.25 & 1.67 & 3.55 & 2.60 & 3.27 & 3.12 & 3.00 & 2.69 & 2.88 & 3.86 & 0.44 & 0.96 & 1.01 & 1.28 & 1.93 & 1.66 & 0.84 & 0.73 & 0.81 \\
\hline Paiements du 1er pilier & 1.67 & 1.53 & 1.68 & 0.04 & 0.03 & 0.04 & 0.17 & 0.20 & 0.25 & 1.38 & 3.42 & 2.36 & 3.32 & 3.04 & 3.14 & 2.04 & 3.03 & 4.28 & 0.45 & 0.49 & 0.83 & 1.28 & 1.35 & 1.34 & 0.83 & 0.73 & 0.80 \\
\hline Paiements du zème pilier & 0.57 & 0.54 & 0.59 & 0.21 & 0.31 & 0.03 & 0.61 & 0.41 & 0.29 & 4.04 & 4.14 & 4.29 & 3.37 & 3.71 & 1.79 & 9.28 & 2.34 & 0.58 & 0.39 & 0.09 & 0.01 & 1.29 & 5.27 & 4.38 & 0.83 & 0.77 & 0.88 \\
\hline Autres paiements & 0.42 & 0.70 & 0.21 & 0.00 & 0.13 & 0.00 & 0.71 & 0.42 & 0.25 & 3.44 & 4.89 & 6.84 & 0.26 & 1.84 & 2.34 & 0.10 & 0.12 & 0.00 & 0.31 & 32.47 & 26.75 & 1.14 & 1.79 & 2.43 & 1.13 & 0.70 & 0.74 \\
\hline Soutien des prix du marché ${ }^{3}$ & 1.27 & 1.15 & 1.68 & 1.91 & 1.63 & 1.78 & 1.30 & 1.42 & 1.15 & 1.51 & 1.65 & 1.27 & 1.04 & 1.02 & 1.23 & 2.51 & 2.46 & 4.44 & 0.41 & 0.61 & 1.61 & 0.39 & 0.37 & 0.38 & 0.66 & 0.70 & 0.60 \\
\hline Recettes brutes ${ }^{4}$ & 0.60 & 0.60 & 0.71 & 1.96 & 1.48 & 1.33 & 0.76 & 0.82 & 0.89 & 2.96 & 3.22 & 3.01 & 2.17 & 2.21 & 2.19 & 11.48 & 9.15 & 12.40 & 6.04 & 5.79 & 8.23 & 0.95 & 1.09 & 1.05 & 0.80 & 0.75 & 0.74 \\
\hline Dépenses d'exploitation & 0.71 & 0.72 & 0.78 & 2.20 & 1.70 & 1.62 & 0.67 & 0.74 & 0.79 & 2.65 & 2.85 & 2.92 & 2.27 & 2.36 & 2.42 & 15.06 & 10.13 & 12.13 & 6.28 & 6.03 & 10.69 & 0.66 & 0.79 & 0.74 & 0.78 & 0.74 & 0.74 \\
\hline Revenu net d'exploitation ${ }^{5}$ & -0.79 & -0.82 & 0.08 & -1.17 & -1.12 & -1.29 & 1.89 & 1.76 & 1.86 & 7.08 & 7.54 & 3.80 & 0.86 & 0.42 & 0.09 & -35.33 & -2.44 & 14.88 & 2.88 & 2.97 & -14.28 & 4.74 & 4.68 & 3.90 & 1.04 & 0.79 & 0.74 \\
\hline Amortissements ${ }^{6}$ & 0.78 & 0.81 & 0.84 & 1.73 & 1.34 & 1.36 & 0.84 & 0.84 & 0.96 & 2.19 & 2.32 & 2.27 & 1.31 & 1.47 & 1.45 & 4.43 & 3.52 & 6.51 & 2.49 & 2.44 & 3.35 & 0.68 & 0.88 & 0.75 & 0.92 & 0.89 & 0.85 \\
\hline Revenu agricole $^{7}$ & 3.47 & 4.67 & 7.33 & 6.71 & 7.19 & 24.29 & -0.95 & -1.32 & -6.75 & -6.22 & -10.03 & -10.89 & 2.07 & 3.95 & 13.14 & 72.70 & 17.65 & -65.70 & 1.81 & 1.19 & 155.36 & -6.29 & -8.13 & -26.44 & 0.71 & 1.14 & 1.78 \\
\hline Revenu non agricole ${ }^{8}$ & n.a. & n.a. & n.a. & n.a. & n.a. & n.a. & n.a. & n.a. & n.a. & n.a. & n.a. & n.a. & n.a. & n.a. & n.a. & n.a. & n.a. & n.a. & n.a. & n.a. & n.a. & n.a. & n.a. & n.a. & n.a. & n.a. & n.a. \\
\hline Revenu des ménages agricoles ${ }^{9}$ & n.a. & n.a. & n.a. & n.a. & n.a. & n.a. & n.a. & n.a. & n.a. & n.a. & n.a. & n.a. & n.a. & n.a. & n.a. & n.a. & n.a. & n.a. & n.a. & n.a. & n.a. & n.a. & n.a. & n.a. & n.a. & n.a. & n.a. \\
\hline Total des actifs agricoles ${ }^{11}$ & n.a. & n.a. & n.a. & n.a. & n.a. & n.a. & n.a. & n.a. & n.a. & n.a. & n.a. & n.a. & n.a. & n.a. & n.a. & n.a. & n.a. & n.a. & n.a. & n.a. & n.a. & n.a. & n.a. & n.a. & n.a. & n.a. & n.a. \\
\hline Total des dettes agricoles ${ }^{12}$ & n.a. & n.a. & n.a. & n.a. & n.a. & n.a. & n.a. & n.a. & n.a. & n.a. & n.a. & n.a. & n.a. & n.a. & n.a. & n.a. & n.a. & n.a. & n.a. & n.a. & n.a. & n.a. & n.a. & n.a. & n.a. & n.a. & n.a. \\
\hline Capitaux propres agricoles ${ }^{13}$ & n.a. & n.a. & n.a. & n.a. & n.a. & n.a. & n.a. & n.a. & n.a. & n.a. & n.a. & n.a. & n.a. & n.a. & n.a. & n.a. & n.a. & n.a. & n.a. & n.a. & n.a. & n.a. & n.a. & n.a. & n.a. & n.a. & n.a. \\
\hline
\end{tabular}

Notes identiques à celles du tableau $\mathrm{C} 1$.

Source : tableaux transmis par les participants. 
Tableau annexe C6. Part du soutien dans les recettes des exploitations et dans le revenu agricole,

\begin{tabular}{|c|c|c|c|c|c|c|c|c|c|c|}
\hline \multirow{2}{*}{$\begin{array}{l}\text { Pays } \\
\text { Type d'exploitation }\end{array}$} & \multicolumn{10}{|c|}{ Canada } \\
\hline & $\begin{array}{l}\text { Toutes } \\
\text { exploit. }\end{array}$ & $\begin{array}{l}\text { Grandes } \\
\text { cultures }\end{array}$ & $\begin{array}{l}\text { Pépinières, } \\
\text { serres et } \\
\text { floriculture }\end{array}$ & $\begin{array}{l}\text { Fruits et } \\
\text { légumes }\end{array}$ & Lait & Bovins & Porcins & $\begin{array}{l}\text { Volaille/ } \\
\text { oeufs }\end{array}$ & $\begin{array}{l}\text { Ovins/ } \\
\text { caprins }\end{array}$ & Autres \\
\hline Part du soutien dans les recettes brutes ${ }^{1}$ & 13 & 11 & 4 & 7 & 43 & 7 & 11 & 9 & n.a. & 10 \\
\hline Tous paiements $^{2}$ & 7 & 11 & 4 & 7 & 3 & 7 & 11 & 1 & n.a. & 8 \\
\hline Paiements du 1er pilier & n.a. & n.a. & n.a. & n.a. & n.a. & n.a. & n.a. & n.a. & n.a. & n.a. \\
\hline Paiements du 2ème pilier & n.a. & n.a. & n.a. & n.a. & n.a. & n.a. & n.a. & n.a. & n.a. & n.a. \\
\hline Autres paiements & 7 & 11 & 4 & 7 & 3 & 7 & 11 & 1 & n.a. & 8 \\
\hline Soutien des prix du marché ${ }^{3}$ & 6 & 0 & 0 & 0 & 40 & 0 & 0 & 7 & n.a. & 2 \\
\hline Part du soutien dans le revenu agricole & 211 & 121 & 159 & 77 & 390 & 295 & 994 & 129 & n.a. & 134 \\
\hline Pays & \multicolumn{10}{|c|}{ États-Unis } \\
\hline Type d'exploitation & $\begin{array}{l}\text { Toutes } \\
\text { exploit. }\end{array}$ & $\begin{array}{l}\text { Grandes } \\
\text { cultures }\end{array}$ & $\begin{array}{c}\text { Pépinières, } \\
\text { serres et } \\
\text { floriculture }\end{array}$ & $\begin{array}{l}\text { Fruits et } \\
\text { légumes }\end{array}$ & Lait & Bovins & Porcins & $\begin{array}{l}\text { Volaille/ } \\
\text { oeufs }\end{array}$ & $\begin{array}{l}\text { Ovins/ } \\
\text { caprins }\end{array}$ & Autres \\
\hline Part du soutien dans les recettes brutes ${ }^{1}$ & 7 & 7 & 0 & 1 & 22 & 2 & 1 & 1 & 9 & 8 \\
\hline Tous paiements $^{2}$ & 3 & 7 & 0 & 1 & 1 & 2 & 1 & 1 & 3 & 3 \\
\hline Paiements du 1er pilier & 0 & 0 & 0 & 0 & 0 & 0 & 0 & 0 & 0 & 0 \\
\hline Paiements du 2ème pilier & 0 & 0 & 0 & 0 & 0 & 0 & 0 & 0 & 0 & 0 \\
\hline Autres paiements & 0 & 0 & 0 & 0 & 0 & 0 & 0 & 0 & 0 & 0 \\
\hline Soutien des prix du marché ${ }^{3}$ & 4 & 0 & 0 & 0 & 21 & 0 & 0 & 0 & 7 & 5 \\
\hline Part du soutien dans le revenu agricole & 33 & 31 & 1 & 5 & 104 & 21 & 5 & 2 & 49 & 41 \\
\hline Pays & \multicolumn{10}{|c|}{ UE27 } \\
\hline Type d'exploitation & $\begin{array}{l}\text { Toutes } \\
\text { exploit. }\end{array}$ & $\begin{array}{l}\text { Grandes } \\
\text { cultures }\end{array}$ & $\begin{array}{l}\text { Pépinières, } \\
\text { serres et } \\
\text { floriculture } \\
\end{array}$ & $\begin{array}{l}\text { Fruits et } \\
\text { légumes }\end{array}$ & Lait & Bovins & Porcins & $\begin{array}{l}\text { Volaille/ } \\
\text { oeufs }\end{array}$ & $\begin{array}{l}\text { Ovins/ } \\
\text { caprins }\end{array}$ & Autres \\
\hline Part du soutien dans les recettes brutes ${ }^{1}$ & 20 & 25 & 3 & 8 & 21 & 45 & 5 & 20 & 34 & 17 \\
\hline Tous paiements $^{2}$ & 14 & 19 & 1 & 7 & 14 & 29 & 4 & 2 & 25 & 12 \\
\hline Paiements du 1er pilier & 12 & 17 & 1 & 6 & 11 & 22 & 3 & 2 & 17 & 10 \\
\hline Paiements du 2ème pilier & 2 & 2 & 0 & 1 & 3 & 7 & 1 & 0 & 8 & 2 \\
\hline Autres paiements & 0 & 0 & 0 & 0 & 0 & 0 & 0 & 0 & 0 & 0 \\
\hline Soutien des prix du marché ${ }^{3}$ & 6 & 6 & 2 & 1 & 7 & 16 & 1 & 18 & 9 & 5 \\
\hline Part du soutien dans le revenu agricole & 71 & 81 & 19 & 20 & 75 & 166 & 36 & 136 & 102 & 65 \\
\hline Pays & \multicolumn{10}{|c|}{ Danemark } \\
\hline Type d'exploitation & $\begin{array}{l}\text { Toutes } \\
\text { exploit. }\end{array}$ & $\begin{array}{l}\text { Grandes } \\
\text { cultures }\end{array}$ & $\begin{array}{c}\text { Pépinières, } \\
\text { serres et } \\
\text { floriculture }\end{array}$ & $\begin{array}{l}\text { Fruits et } \\
\text { légumes }\end{array}$ & Lait & Bovins & Porcins & $\begin{array}{l}\text { Volaille/ } \\
\text { oeufs }\end{array}$ & $\begin{array}{l}\text { Ovins/ } \\
\text { caprins }\end{array}$ & Autres \\
\hline Part du soutien dans les recettes brutes ${ }^{1}$ & 22 & 13 & n.a. & n.a. & 69 & n.a. & 21 & 16 & n.a. & n.a. \\
\hline Tous paiements $^{2}$ & 8 & 13 & n.a. & n.a. & 10 & n.a. & 5 & 6 & n.a. & n.a. \\
\hline Paiements du 1er pilier & 8 & 13 & n.a. & n.a. & 10 & n.a. & 5 & 6 & n.a. & n.a. \\
\hline Paiements du 2ème pilier & 0 & 0 & n.a. & n.a. & 0 & n.a. & 0 & 0 & n.a. & n.a. \\
\hline Autres paiements & 0 & 0 & n.a. & n.a. & 0 & n.a. & 0 & 0 & n.a. & n.a. \\
\hline Soutien des prix du marché ${ }^{3}$ & 13 & 1 & n.a. & n.a. & 59 & n.a. & 16 & 11 & n.a. & n.a. \\
\hline Part du soutien dans le revenu agricole & 336 & 81 & n.a. & n.a. & 359 & n.a. & -125 & -1601 & n.a. & n.a. \\
\hline
\end{tabular}




\begin{tabular}{|c|c|c|c|c|c|c|c|c|c|c|}
\hline \multirow{2}{*}{$\begin{array}{l}\text { Pays } \\
\text { Type d'exploitation }\end{array}$} & \multicolumn{10}{|c|}{ Angleterre } \\
\hline & $\begin{array}{l}\text { Toutes } \\
\text { exploit. }\end{array}$ & $\begin{array}{l}\text { Grandes } \\
\text { cultures }\end{array}$ & $\begin{array}{c}\text { Pépinières, } \\
\text { serres et } \\
\text { floriculture }\end{array}$ & $\begin{array}{l}\text { Fruits et } \\
\text { légumes }\end{array}$ & Lait & Bovins & Porcins & $\begin{array}{c}\text { Volaille/ } \\
\text { oeufs }\end{array}$ & $\begin{array}{l}\text { Ovins/ } \\
\text { caprins }\end{array}$ & Autres \\
\hline Part du soutien dans les recettes brutes ${ }^{1}$ & 28 & 23 & 2 & 4 & 38 & 63 & 27 & 17 & 57 & 26 \\
\hline Tous paiements $^{2}$ & 14 & 15 & 0 & 3 & 9 & 31 & 2 & 1 & 35 & 16 \\
\hline Paiements du 1er pilier & 11 & 12 & 0 & 2 & 8 & 23 & 1 & 1 & 24 & 13 \\
\hline Paiements du 2ème pilier & 3 & 3 & 0 & 1 & 1 & 7 & 0 & 0 & 11 & 3 \\
\hline Autres paiements & 0 & 0 & 0 & 0 & 0 & 1 & 0 & 0 & 0 & 0 \\
\hline Soutien des prix du marché ${ }^{3}$ & 14 & 8 & 1 & 1 & 28 & 32 & 25 & 16 & 22 & 9 \\
\hline Part du soutien dans le revenu agricole & 129 & 93 & 10 & 21 & 190 & 338 & 1495 & 85 & 336 & 105 \\
\hline Pays & \multicolumn{10}{|c|}{ Estonie } \\
\hline Type d'exploitation & $\begin{array}{l}\text { Toutes } \\
\text { exploit. }\end{array}$ & $\begin{array}{l}\text { Grandes } \\
\text { cultures }\end{array}$ & $\begin{array}{c}\text { Pépinières, } \\
\text { serres et } \\
\text { floriculture }\end{array}$ & $\begin{array}{l}\text { Fruits et } \\
\text { légumes }\end{array}$ & Lait & Bovins & Porcins & $\begin{array}{c}\text { Volaille/ } \\
\text { oeufs }\end{array}$ & $\begin{array}{l}\text { Ovins/ } \\
\text { caprins }\end{array}$ & Autres \\
\hline Part du soutien dans les recettes brutes ${ }^{1}$ & 23 & 23 & 8 & 12 & 22 & 57 & 1 & n.a. & 50 & 26 \\
\hline Tous paiements $^{2}$ & 19 & 21 & 6 & 9 & 17 & 50 & 2 & n.a. & 43 & 22 \\
\hline Paiements du 1er pilier & 12 & 14 & 0 & 4 & 12 & 18 & 0 & n.a. & 17 & 12 \\
\hline Paiements du 2ème pilier & 8 & 8 & 6 & 4 & 6 & 32 & 2 & n.a. & 27 & 10 \\
\hline Autres paiements & 0 & 0 & 0 & 0 & 0 & 0 & 0 & n.a. & 0 & 0 \\
\hline Soutien des prix du marché ${ }^{3}$ & 3 & 2 & 2 & 4 & 4 & 8 & -1 & n.a. & 7 & 4 \\
\hline Part du soutien dans le revenu agricole & 95 & 62 & 63 & 155 & 132 & 131 & -26 & n.a. & 186 & 113 \\
\hline Pays & \multicolumn{10}{|c|}{ Espagne } \\
\hline Type d'exploitation & $\begin{array}{l}\text { Toutes } \\
\text { exploit. }\end{array}$ & $\begin{array}{l}\text { Grandes } \\
\text { cultures }\end{array}$ & $\begin{array}{c}\text { Pépinières, } \\
\text { serres et } \\
\text { floriculture } \\
\end{array}$ & $\begin{array}{l}\text { Fruits et } \\
\text { légumes }\end{array}$ & Lait & Bovins & Porcins & $\begin{array}{l}\text { Volaille/ } \\
\text { oeufs }\end{array}$ & $\begin{array}{l}\text { Ovins/ } \\
\text { caprins }\end{array}$ & Autres \\
\hline Part du soutien dans les recettes brutes ${ }^{1}$ & n.a. & n.a. & n.a. & n.a. & n.a. & n.a. & n.a. & n.a. & n.a. & n.a. \\
\hline Tous paiements $^{2}$ & 12 & 23 & 1 & 5 & 8 & 24 & 2 & 2 & 15 & 14 \\
\hline Paiements du 1er pilier & n.a. & n.a. & n.a. & n.a. & n.a. & n.a. & n.a. & n.a. & n.a. & n.a. \\
\hline Paiements du 2ème pilier & n.a. & n.a. & n.a. & n.a. & n.a. & n.a. & n.a. & n.a. & n.a. & n.a. \\
\hline Autres paiements & n.a. & n.a. & n.a. & n.a. & n.a. & n.a. & n.a. & n.a. & n.a. & n.a. \\
\hline Soutien des prix du marché ${ }^{3}$ & n.a. & n.a. & n.a. & n.a. & n.a. & n.a. & n.a. & n.a. & n.a. & n.a. \\
\hline Part du soutien dans le revenu agricole & n.a. & n.a. & n.a. & n.a. & n.a. & n.a. & n.a. & n.a. & n.a. & n.a. \\
\hline Pays & \multicolumn{10}{|c|}{ Flandre belge } \\
\hline Type d'exploitation & $\begin{array}{l}\text { Toutes } \\
\text { exploit. }\end{array}$ & $\begin{array}{l}\text { Grandes } \\
\text { cultures }\end{array}$ & $\begin{array}{c}\text { Pépinières, } \\
\text { serres et } \\
\text { floriculture } \\
\end{array}$ & $\begin{array}{l}\text { Fruits et } \\
\text { légumes }\end{array}$ & Lait & Bovins & Porcins & $\begin{array}{l}\text { Volaille/ } \\
\text { oeufs }\end{array}$ & $\begin{array}{l}\text { Ovins/ } \\
\text { caprins }\end{array}$ & Autres \\
\hline Part du soutien dans les recettes brutes ${ }^{1}$ & 14 & 26 & 6 & 4 & 20 & 34 & 4 & n.a. & n.a. & 17 \\
\hline Tous paiements $^{2}$ & 7 & 11 & 2 & 1 & 11 & 14 & 2 & n.a. & n.a. & 10 \\
\hline Paiements du 1er pilier & 5 & 8 & 0 & 0 & 9 & 13 & 1 & n.a. & n.a. & 7 \\
\hline Paiements du 2ème pilier & 2 & 3 & 1 & 1 & 2 & 2 & 1 & n.a. & n.a. & 2 \\
\hline Autres paiements & 0 & 1 & 0 & 0 & 0 & 0 & 0 & n.a. & n.a. & 0 \\
\hline Soutien des prix du marché ${ }^{3}$ & 7 & 15 & 4 & 3 & 8 & 19 & 1 & n.a. & n.a. & 7 \\
\hline Part du soutien dans le revenu agricole & 56 & 81 & 34 & 11 & 50 & 123 & 38 & n.a. & n.a. & 63 \\
\hline Pays & \multicolumn{10}{|c|}{ Pays-Bas } \\
\hline Type d'exploitation & $\begin{array}{l}\text { Toutes } \\
\text { exploit. }\end{array}$ & $\begin{array}{l}\text { Grandes } \\
\text { cultures }\end{array}$ & $\begin{array}{c}\text { Pépinières, } \\
\text { serres et } \\
\text { floriculture } \\
\end{array}$ & $\begin{array}{l}\text { Fruits et } \\
\text { légumes }\end{array}$ & Lait & Bovins & Porcins & $\begin{array}{c}\text { Volaille/ } \\
\text { oeufs }\end{array}$ & $\begin{array}{l}\text { Ovins/ } \\
\text { caprins }\end{array}$ & Autres \\
\hline Part du soutien dans les recettes brutes ${ }^{1}$ & 11 & 16 & 4 & 4 & 18 & 28 & 0 & 14 & n.a. & 12 \\
\hline Tous paiements ${ }^{2}$ & 5 & 8 & 1 & 0 & 10 & 12 & 1 & 0 & n.a. & 4 \\
\hline Paiements du 1er pilier & 4 & 7 & 0 & 0 & 9 & 9 & 1 & 0 & n.a. & 3 \\
\hline Paiements du 2ème pilier & 0 & 1 & 0 & 0 & 1 & 4 & 0 & 0 & n.a. & 0 \\
\hline Autres paiements & 0 & 1 & 1 & 0 & 0 & 0 & 0 & 0 & n.a. & 1 \\
\hline Soutien des prix du marché ${ }^{3}$ & 6 & 8 & 3 & 4 & 8 & 16 & 0 & 14 & n.a. & 8 \\
\hline Part du soutien dans le revenu agricole & 80 & 75 & 39 & 16 & 62 & -6646 & -2 & 148 & n.a. & 118 \\
\hline
\end{tabular}




\begin{tabular}{|c|c|c|c|c|c|c|c|c|c|c|}
\hline \multirow{2}{*}{$\begin{array}{l}\text { Pays } \\
\text { Type d'exploitation }\end{array}$} & \multicolumn{10}{|c|}{ Finlande } \\
\hline & $\begin{array}{l}\text { Toutes } \\
\text { exploit. }\end{array}$ & $\begin{array}{l}\text { Grandes } \\
\text { cultures }\end{array}$ & $\begin{array}{l}\text { Pépinières, } \\
\text { serres et } \\
\text { floriculture }\end{array}$ & $\begin{array}{l}\text { Fruits et } \\
\text { légumes }\end{array}$ & Lait & Bovins & Porcins & $\begin{array}{c}\text { Volaille/ } \\
\text { oeufs }\end{array}$ & $\begin{array}{c}\text { Ovins/ } \\
\text { caprins }\end{array}$ & Autres \\
\hline Part du soutien dans les recettes brutes ${ }^{1}$ & n.a. & n.a. & n.a. & n.a. & n.a. & n.a. & n.a. & n.a. & n.a. & n.a. \\
\hline Tous paiements ${ }^{2}$ & 37 & 43 & 13 & n.a. & 35 & 59 & 24 & n.a. & n.a. & n.a. \\
\hline Paiements du 1er pilier & 10 & 15 & 0 & n.a. & 9 & 18 & 4 & n.a. & n.a. & n.a. \\
\hline Paiements du 2ème pilier & 14 & 23 & 2 & n.a. & 11 & 18 & 7 & n.a. & n.a. & n.a. \\
\hline Autres paiements & 12 & 5 & 11 & n.a. & 15 & 23 & 13 & n.a. & n.a. & n.a. \\
\hline Soutien des prix du marché ${ }^{3}$ & n.a. & n.a. & n.a. & n.a. & n.a. & n.a. & n.a. & n.a. & n.a. & n.a. \\
\hline Part du soutien dans le revenu agricole & n.a. & n.a. & n.a. & n.a. & n.a. & n.a. & n.a. & n.a. & n.a. & n.a. \\
\hline Pays & \multicolumn{10}{|c|}{ France } \\
\hline Type d'exploitation & $\begin{array}{l}\text { Toutes } \\
\text { exploit. }\end{array}$ & $\begin{array}{l}\text { Grandes } \\
\text { cultures }\end{array}$ & $\begin{array}{c}\text { Pépinières, } \\
\text { serres et } \\
\text { floriculture } \\
\end{array}$ & $\begin{array}{l}\text { Fruits et } \\
\text { légumes }\end{array}$ & Lait & Bovins & Porcins & $\begin{array}{l}\text { Volaille/ } \\
\text { oeufs }\end{array}$ & $\begin{array}{c}\text { Ovins/ } \\
\text { caprins }\end{array}$ & Autres \\
\hline Part du soutien dans les recettes brutes ${ }^{1}$ & n.a. & n.a. & n.a. & n.a. & n.a. & n.a. & n.a. & n.a. & n.a. & n.a. \\
\hline Tous paiements ${ }^{2}$ & 16 & 20 & 1 & 3 & 16 & 31 & 3 & 5 & 27 & 17 \\
\hline Paiements du 1er pilier & 13 & 19 & 0 & 2 & 13 & 23 & 2 & 4 & 13 & 15 \\
\hline Paiements du 2ème pilier & 1 & 0 & 0 & 0 & 3 & 6 & 0 & 0 & 10 & 1 \\
\hline Autres paiements & 1 & 2 & 1 & 1 & 1 & 2 & 0 & 1 & 3 & 1 \\
\hline Soutien des prix du marché ${ }^{3}$ & n.a. & n.a. & n.a. & n.a. & n.a. & n.a. & n.a. & n.a. & n.a. & n.a. \\
\hline Part du soutien dans le revenu agricole & n.a. & n.a. & n.a. & n.a. & n.a. & n.a. & n.a. & n.a. & n.a. & n.a. \\
\hline Pays & \multicolumn{10}{|c|}{ Allemagne } \\
\hline Type d'exploitation & $\begin{array}{l}\text { Toutes } \\
\text { exploit. }\end{array}$ & $\begin{array}{l}\text { Grandes } \\
\text { cultures }\end{array}$ & $\begin{array}{c}\text { Pépinières, } \\
\text { serres et } \\
\text { floriculture } \\
\end{array}$ & $\begin{array}{l}\text { Fruits et } \\
\text { légumes }\end{array}$ & Lait & Bovins & Porcins & $\begin{array}{c}\text { Volaille/ } \\
\text { oeufs }\end{array}$ & $\begin{array}{c}\text { Ovins/ } \\
\text { caprins }\end{array}$ & Autres \\
\hline Part du soutien dans les recettes brutes ${ }^{1}$ & 20 & 24 & 3 & 3 & 27 & 33 & 6 & 16 & 58 & 19 \\
\hline Tous paiements ${ }^{2}$ & 14 & 19 & 1 & 2 & 17 & 22 & 5 & 5 & 49 & 14 \\
\hline Paiements du 1er pilier & 11 & 17 & 0 & 1 & 12 & 16 & 4 & 4 & 27 & 11 \\
\hline Paiements du 2ème pilier & 2 & 2 & 0 & 1 & 4 & 6 & 0 & 1 & 22 & 2 \\
\hline Autres paiements & 1 & 1 & 0 & 0 & 1 & 1 & 0 & 0 & 1 & 1 \\
\hline Soutien des prix du marché ${ }^{3}$ & 6 & 5 & 2 & 1 & 11 & 12 & 2 & 11 & 9 & 5 \\
\hline Part du soutien dans le revenu agricole & 121 & 133 & 21 & 13 & 120 & 225 & 53 & 120 & 225 & 156 \\
\hline Pays & \multicolumn{10}{|c|}{ Irlande } \\
\hline Type d'exploitation & $\begin{array}{l}\text { Toutes } \\
\text { exploit. }\end{array}$ & $\begin{array}{l}\text { Grandes } \\
\text { cultures }\end{array}$ & $\begin{array}{c}\text { Pépinières, } \\
\text { serres et } \\
\text { floriculture } \\
\end{array}$ & $\begin{array}{l}\text { Fruits et } \\
\text { légumes }\end{array}$ & Lait & Bovins & Porcins & $\begin{array}{c}\text { Volaille/ } \\
\text { oeufs }\end{array}$ & $\begin{array}{c}\text { Ovins/ } \\
\text { caprins }\end{array}$ & Autres \\
\hline Part du soutien dans les recettes brutes ${ }^{1}$ & 38 & 30 & n.a. & n.a. & 20 & 69 & n.a. & n.a. & 49 & n.a. \\
\hline Tous paiements ${ }^{2}$ & 31 & 25 & n.a. & n.a. & 16 & 49 & n.a. & n.a. & 49 & n.a. \\
\hline Paiements du 1er pilier & 21 & 21 & n.a. & n.a. & 13 & 32 & n.a. & n.a. & 11 & n.a. \\
\hline Paiements du 2ème pilier & 10 & 4 & n.a. & n.a. & 4 & 17 & n.a. & n.a. & 8 & n.a. \\
\hline Autres paiements & 0 & 0 & n.a. & n.a. & 0 & 0 & n.a. & n.a. & 0 & n.a. \\
\hline Soutien des prix du marché ${ }^{3}$ & 7 & 4 & n.a. & n.a. & 3 & 20 & n.a. & n.a. & 4 & n.a. \\
\hline Part du soutien dans le revenu agricole & 103 & 72 & n.a. & n.a. & 52 & 216 & n.a. & n.a. & 173 & n.a. \\
\hline Pays & \multicolumn{10}{|c|}{ Italie } \\
\hline Type d'exploitation & $\begin{array}{l}\text { Toutes } \\
\text { exploit. }\end{array}$ & $\begin{array}{l}\text { Grandes } \\
\text { cultures }\end{array}$ & $\begin{array}{c}\text { Pépinières, } \\
\text { serres et } \\
\text { floriculture }\end{array}$ & $\begin{array}{l}\text { Fruits et } \\
\text { légumes }\end{array}$ & Lait & Bovins & Porcins & $\begin{array}{c}\text { Volaille/ } \\
\text { oeufs }\end{array}$ & $\begin{array}{l}\text { Ovins/ } \\
\text { caprins }\end{array}$ & Autres \\
\hline Part du soutien dans les recettes brutes ${ }^{1}$ & 14 & 32 & 7 & 9 & 10 & 15 & 5 & 2 & 16 & 14 \\
\hline Tous paiements $^{2}$ & 9 & 20 & 0 & 3 & 8 & 13 & 3 & 1 & 15 & 10 \\
\hline Paiements du 1er pilier & 8 & 19 & 0 & 2 & 6 & 12 & 3 & 1 & 10 & 9 \\
\hline Paiements du 2ème pilier & 1 & 1 & 0 & 0 & 1 & 1 & 0 & 0 & 4 & 1 \\
\hline Autres paiements & 0 & 0 & 0 & 0 & 0 & 0 & 0 & 0 & 0 & 0 \\
\hline Soutien des prix du marché ${ }^{3}$ & 5 & 11 & 6 & 6 & 2 & 3 & 2 & 1 & 2 & 4 \\
\hline Part du soutien dans le revenu agricole & -1228 & -268 & -32 & 102 & 242 & -224 & 76 & -10 & 57 & -508 \\
\hline
\end{tabular}




\section{Notes au tableau annexe C6}

Les exploitations incluses représentent au total $90 \%$ de la production agricole nationale.

1. Inclut les recettes tirées des ventes de produits et de services agricoles tels que le travail à façon ou la mise en location de terres, de quotas, de bâtiments ou de machines, entre autres.

2. Inclut les transferts budgétaires aux agriculteurs résultant de la politique agricole et des paiements des systèmes d'assurance, des programmes de conservation des terres aux États-Unis et des premier et second piliers de la Politique agricole commune de l'Union européenne.

3. Le soutien des prix du marché (SPM) est calculé en appliquant le ratio SPM de chaque produit de base, lorsqu'il existe dans la base de données des ESP de l'OCDE, aux recettes de l'exploitation tirées de ce produit. Le ratio SPM moyen de l'ensemble des produits de la base ESP est utilisé pour les recettes provenant de produits pour lesquels il n'existe pas de ratio SPM.

Source : tableaux transmis par les participants. 
Tableau annexe C7. Ratio de la moyenne des exploitations regroupées par type de régions, sur la moyenne de l'ensemble des exploitations, certains pays de l'OCDE, 2004, 2006, 2007

\begin{tabular}{|c|c|c|c|c|c|c|c|c|c|}
\hline \multirow{3}{*}{$\begin{array}{l}\text { Pays } \\
\text { Régions } \\
\text { Années }\end{array}$} & \multicolumn{9}{|c|}{ Canada } \\
\hline & \multicolumn{3}{|c|}{ Essentiellement rurales/Toutes } & \multicolumn{3}{|c|}{ Intermédiaires/Toutes } & \multicolumn{3}{|c|}{ Essentiellement urbaines/Toutes } \\
\hline & 2004 & 2006 & 2007 & 2004 & 2006 & 2007 & 2004 & 2006 & 2007 \\
\hline Exploitations de la population & 0.8 & 0.8 & 0.8 & 0.1 & 0.1 & 0.2 & 0.1 & 0.1 & 0.1 \\
\hline Unité de travail (éq. temps plein) & n.a. & n.a. & n.a. & n.a. & n.a. & n.a. & n.a. & n.a. & n.a. \\
\hline Superficie agricole utilisée & 0.9 & 0.9 & 0.9 & 0.1 & 0.1 & 0.1 & 0.0 & 0.0 & 0.0 \\
\hline Production agricole brute ${ }^{1}$ & 0.9 & 0.9 & 1.0 & 1.1 & 1.1 & 1.0 & 1.5 & 1.6 & 1.5 \\
\hline Soutien total & 1.0 & 1.0 & 1.0 & 1.1 & 1.1 & 1.1 & 0.9 & 1.0 & 1.0 \\
\hline Tous paiements ${ }^{2}$ & 1.0 & 1.0 & 1.0 & 1.0 & 1.0 & 1.0 & 0.8 & 0.9 & 0.8 \\
\hline Paiements du 1er pilier & n.a. & n.a. & n.a. & n.a. & n.a. & n.a. & n.a. & n.a. & n.a. \\
\hline Paiements du 2ème pilier & n.a. & n.a. & n.a. & n.a. & n.a. & n.a. & n.a. & n.a. & n.a. \\
\hline Autres paiements & 1.0 & 1.0 & 1.0 & 1.0 & 1.0 & 1.0 & 0.8 & 0.9 & 0.8 \\
\hline Soutien des prix du marché ${ }^{3}$ & 0.9 & 0.9 & 0.9 & 1.3 & 1.3 & 1.2 & 1.1 & 1.2 & 1.2 \\
\hline Recettes brutes ${ }^{4}$ & 0.9 & 0.9 & 1.0 & 1.1 & 1.1 & 1.0 & 1.5 & 1.5 & 1.4 \\
\hline Dépenses d'exploitation & 0.9 & 0.9 & 0.9 & 1.1 & 1.1 & 1.1 & 1.6 & 1.6 & 1.5 \\
\hline Revenu net d'exploitation ${ }^{5}$ & 1.0 & 1.0 & 1.0 & 1.0 & 1.1 & 1.0 & 0.8 & 1.0 & 0.9 \\
\hline Amortissements $^{6}$ & 1.0 & 1.0 & 1.0 & 1.0 & 1.0 & 1.0 & 1.1 & 1.2 & 1.1 \\
\hline Revenu agricole $^{7}$ & 1.1 & 1.0 & 1.0 & 1.0 & 1.3 & 1.0 & 0.4 & 0.7 & 0.7 \\
\hline Revenu non agricole $^{8}$ & 1.0 & 1.0 & 0.9 & 1.0 & 1.0 & 1.0 & 1.4 & 1.5 & 1.9 \\
\hline Revenu des ménages agricoles ${ }^{9}$ & 1.0 & 1.0 & 1.0 & 1.0 & 1.1 & 1.0 & 0.9 & 1.2 & 1.3 \\
\hline Total des actifs agricoles ${ }^{11}$ & 1.0 & 0.9 & 1.0 & 1.0 & 1.2 & 1.0 & 1.3 & 1.3 & 1.3 \\
\hline Total des dettes agricoles ${ }^{12}$ & 1.0 & 1.0 & 1.0 & 1.1 & 1.2 & 1.1 & 1.0 & 1.0 & 1.2 \\
\hline Capitaux propres agricoles ${ }^{13}$ & 1.0 & 0.9 & 1.0 & 1.0 & 1.1 & 1.0 & 1.3 & 1.4 & 1.3 \\
\hline \multirow{3}{*}{$\begin{array}{l}\text { Pays } \\
\text { Régions } \\
\text { Années }\end{array}$} & \multicolumn{9}{|c|}{ États-Unis } \\
\hline & \multicolumn{3}{|c|}{ Essentiellement rurales/Toutes } & \multicolumn{3}{|c|}{ Intermédiaires/Toutes } & \multicolumn{3}{|c|}{ Essentiellement urbaines/Toutes } \\
\hline & 2004 & 2006 & 2007 & 2004 & 2006 & 2007 & 2004 & 2006 & 2007 \\
\hline Exploitations de la population & n.a. & 0.4 & 0.4 & n.a. & 0.2 & 0.2 & n.a. & 0.3 & 0.3 \\
\hline Unité de travail (éq. temps plein) & n.a. & n.a. & n.a. & n.a. & n.a. & n.a. & n.a. & n.a. & n.a. \\
\hline Superficie agricole utilisée & n.a. & 0.6 & 0.6 & n.a. & 0.2 & 0.2 & n.a. & 0.2 & 0.2 \\
\hline Production agricole brute ${ }^{1}$ & n.a. & 0.8 & 0.9 & n.a. & 0.9 & 0.9 & n.a. & 1.3 & 1.2 \\
\hline Soutien total & n.a. & 0.9 & 0.8 & n.a. & 1.1 & 1.0 & n.a. & 1.0 & 1.2 \\
\hline Tous paiements $^{2}$ & n.a. & 1.0 & 1.2 & n.a. & 1.1 & 1.0 & n.a. & 0.9 & 0.8 \\
\hline Paiements du 1er pilier & n.a. & n.a. & n.a. & n.a. & n.a. & n.a. & n.a. & n.a. & n.a. \\
\hline Paiements du 2ème pilier & n.a. & n.a. & n.a. & n.a. & n.a. & n.a. & n.a. & n.a. & n.a. \\
\hline Autres paiements & n.a. & n.a. & n.a. & n.a. & n.a. & n.a. & n.a. & n.a. & n.a. \\
\hline Soutien des prix du marché ${ }^{3}$ & n.a. & 0.6 & 0.5 & n.a. & 1.3 & 1.0 & n.a. & 1.4 & 1.5 \\
\hline Recettes brutes ${ }^{4}$ & n.a. & 0.8 & 0.9 & n.a. & 0.9 & 0.9 & n.a. & 1.3 & 1.2 \\
\hline Dépenses d'exploitation & n.a. & 0.8 & 0.9 & n.a. & 0.9 & 1.0 & n.a. & 1.3 & 1.2 \\
\hline Revenu net d'exploitation 5 & n.a. & 0.8 & 0.9 & n.a. & 0.9 & 0.8 & n.a. & 1.3 & 1.2 \\
\hline Amortissements $^{6}$ & n.a. & 0.9 & 1.0 & n.a. & 1.0 & 1.0 & n.a. & 1.1 & 1.0 \\
\hline Revenu agricole $^{7}$ & n.a. & 0.8 & 0.9 & n.a. & 0.9 & 0.8 & n.a. & 1.4 & 1.3 \\
\hline Revenu non agricole $^{8}$ & n.a. & 0.9 & 1.0 & n.a. & 1.0 & 0.9 & n.a. & 1.1 & 1.1 \\
\hline Revenu des ménages agricoles ${ }^{9}$ & n.a. & 0.8 & 0.9 & n.a. & 0.9 & 0.8 & n.a. & 1.3 & 1.2 \\
\hline Total des actifs agricoles ${ }^{11}$ & n.a. & 0.9 & 1.0 & n.a. & 0.8 & 0.9 & n.a. & 1.3 & 1.1 \\
\hline Total des dettes agricoles ${ }^{12}$ & n.a. & 0.9 & 1.0 & n.a. & 1.0 & 1.0 & n.a. & 1.1 & 1.0 \\
\hline Capitaux propres agricoles ${ }^{13}$ & n.a. & 0.9 & 1.0 & n.a. & 0.8 & 0.9 & n.a. & 1.3 & 1.1 \\
\hline
\end{tabular}




\begin{tabular}{|c|c|c|c|c|c|c|c|c|c|}
\hline \multirow{3}{*}{$\begin{array}{l}\text { Pays } \\
\text { Régions } \\
\text { Années }\end{array}$} & \multicolumn{9}{|c|}{ Angleterre } \\
\hline & \multicolumn{3}{|c|}{ Essentiellement rurales/Toutes } & \multicolumn{3}{|c|}{ Intermédiaires/Toutes } & \multicolumn{3}{|c|}{ Essentiellement urbaines/Toutes } \\
\hline & 2004 & 2006 & 2007 & 2004 & 2006 & 2007 & 2004 & 2006 & 2007 \\
\hline Exploitations de la population & n.a. & 0.9 & 0.9 & n.a. & 0.1 & 0.1 & n.a. & 0.0 & 0.0 \\
\hline Unité de travail (éq. temps plein) & n.a. & 0.9 & 0.9 & n.a. & 0.1 & 0.1 & n.a. & 0.1 & 0.1 \\
\hline Superficie agricole utilisée & n.a. & 0.9 & 0.9 & n.a. & 0.1 & 0.1 & n.a. & 0.0 & 0.0 \\
\hline Production agricole brute ${ }^{1}$ & n.a. & 1.0 & 1.0 & n.a. & 1.3 & 1.2 & n.a. & 1.3 & 1.2 \\
\hline Soutien total & n.a. & 1.0 & 1.0 & n.a. & 0.9 & 0.9 & n.a. & 0.7 & 0.7 \\
\hline Tous paiements ${ }^{2}$ & n.a. & 1.0 & 1.0 & n.a. & 1.0 & 1.0 & n.a. & 0.7 & 0.6 \\
\hline Paiements du 1er pilier & n.a. & 1.0 & 1.0 & n.a. & 1.0 & 1.0 & n.a. & 0.7 & 0.6 \\
\hline Paiements du 2ème pilier & n.a. & 1.0 & 1.0 & n.a. & 1.0 & 0.8 & n.a. & 0.6 & 0.6 \\
\hline Autres paiements & n.a. & 1.1 & 1.0 & n.a. & 0.0 & 0.4 & n.a. & 0.1 & 1.2 \\
\hline Soutien des prix du marché ${ }^{3}$ & n.a. & 1.0 & 1.0 & n.a. & 0.8 & 0.8 & n.a. & 0.8 & 0.7 \\
\hline Recettes brutes ${ }^{4}$ & n.a. & 1.0 & 1.0 & n.a. & 1.2 & 1.2 & n.a. & 1.2 & 1.1 \\
\hline Dépenses d'exploitation & n.a. & 1.0 & 1.0 & n.a. & 1.3 & 1.2 & n.a. & 1.3 & 1.2 \\
\hline Revenu net d'exploitation 5 & n.a. & 1.0 & 1.0 & n.a. & 1.1 & 1.1 & n.a. & 1.0 & 0.9 \\
\hline Amortissements ${ }^{6}$ & n.a. & 1.0 & 1.0 & n.a. & 1.3 & 1.3 & n.a. & 1.0 & 0.9 \\
\hline Revenu agricole $^{7}$ & n.a. & 1.0 & 1.0 & n.a. & 1.1 & 1.1 & n.a. & 0.9 & 0.9 \\
\hline Revenu non agricole ${ }^{8}$ & n.a. & n.a. & n.a. & n.a. & n.a. & n.a. & n.a. & n.a. & n.a. \\
\hline Revenu des ménages agricoles 9 & n.a. & n.a. & n.a. & n.a. & n.a. & n.a. & n.a. & n.a. & n.a. \\
\hline Total des actifs agricoles ${ }^{11}$ & n.a. & 1.0 & 1.0 & n.a. & 1.0 & 1.1 & n.a. & 0.8 & 0.7 \\
\hline Total des dettes agricoles ${ }^{12}$ & n.a. & 1.0 & 1.0 & n.a. & 1.1 & 1.0 & n.a. & 0.9 & 0.8 \\
\hline Capitaux propres agricoles ${ }^{13}$ & n.a. & 1.0 & 1.0 & n.a. & 1.0 & 1.1 & n.a. & 0.8 & 0.7 \\
\hline Pays & & & & & Ilemag & & & & \\
\hline Régions & Essent & ment ru & s/Toutes & Int & édiaires/ & tes & Essentiel & ent urb & s/Toutes \\
\hline Années & 2004 & 2006 & 2007 & 2004 & 2006 & 2007 & 2004 & 2006 & 2007 \\
\hline Exploitations de la population & 0.2 & 0.2 & 0.3 & 0.4 & 0.4 & 0.4 & 0.3 & 0.3 & 0.3 \\
\hline Unité de travail (éq. temps plein) & 0.3 & 0.3 & 0.3 & 0.4 & 0.4 & 0.4 & 0.3 & 0.3 & 0.3 \\
\hline Superficie agricole utilisée & 0.3 & 0.3 & 0.3 & 0.4 & 0.4 & 0.4 & 0.3 & 0.3 & 0.3 \\
\hline Production agricole brute ${ }^{1}$ & 1.2 & 1.2 & 1.2 & 1.0 & 1.0 & 1.0 & 0.9 & 0.9 & 0.9 \\
\hline Soutien total & 1.0 & 1.0 & 1.0 & 1.0 & 1.0 & 1.0 & 1.0 & 1.1 & 1.0 \\
\hline Tous paiements ${ }^{2}$ & 1.0 & 1.0 & 1.0 & 1.0 & 1.0 & 1.0 & 1.0 & 1.1 & 1.1 \\
\hline Paiements du 1er pilier & 1.0 & 1.0 & 1.0 & 1.0 & 1.0 & 1.0 & 0.9 & 1.0 & 1.0 \\
\hline Paiements du 2ème pilier & 0.9 & 0.8 & 0.8 & 0.9 & 0.9 & 0.9 & 1.2 & 1.3 & 1.3 \\
\hline Autres paiements & 1.0 & 1.0 & 1.0 & 0.9 & 0.9 & 0.9 & 1.1 & 1.1 & 1.1 \\
\hline Soutien des prix du marché ${ }^{3}$ & 1.0 & 1.1 & 1.1 & 0.9 & 0.9 & 0.9 & 1.0 & 1.0 & 1.0 \\
\hline Recettes brutes 4 & 1.2 & 1.2 & 1.2 & 1.0 & 1.0 & 1.0 & 0.9 & 0.9 & 0.9 \\
\hline Dépenses d'exploitation & 1.2 & 1.2 & 1.2 & 1.0 & 1.0 & 1.0 & 0.9 & 0.9 & 0.9 \\
\hline Revenu net d'exploitation 5 & 1.2 & 1.1 & 1.1 & 1.0 & 0.9 & 1.0 & 0.9 & 1.0 & 1.0 \\
\hline Amortissements $^{6}$ & 1.1 & 1.1 & 1.0 & 1.0 & 1.0 & 1.0 & 1.0 & 1.0 & 1.0 \\
\hline Revenu agricole $^{7}$ & 1.2 & 1.2 & 1.2 & 1.0 & 0.9 & 1.0 & 0.9 & 0.9 & 0.9 \\
\hline Revenu non agricole $^{8}$ & n.a. & n.a. & n.a. & n.a. & n.a. & n.a. & n.a. & n.a. & n.a. \\
\hline Revenu des ménages agricoles ${ }^{9}$ & n.a. & n.a. & n.a. & n.a. & n.a. & n.a. & n.a. & n.a. & n.a. \\
\hline Total des actifs agricoles ${ }^{11}$ & 1.0 & 1.0 & 1.0 & 1.0 & 1.0 & 1.0 & 1.0 & 1.0 & 1.0 \\
\hline Total des dettes agricoles ${ }^{12}$ & 1.2 & 1.2 & 1.2 & 1.0 & 0.9 & 0.9 & 0.9 & 0.9 & 0.9 \\
\hline Capitaux propres agricoles ${ }^{13}$ & 1.0 & 1.0 & 1.0 & 1.0 & 1.0 & 1.0 & 1.0 & 1.0 & 1.0 \\
\hline
\end{tabular}




\begin{tabular}{|c|c|c|c|c|c|c|c|c|c|}
\hline \multirow{3}{*}{$\begin{array}{l}\text { Pays } \\
\text { Régions } \\
\text { Années }\end{array}$} & \multicolumn{9}{|c|}{ Italie } \\
\hline & \multicolumn{3}{|c|}{ Essentiellement rurales/Toutes } & \multicolumn{3}{|c|}{ Intermédiaires/Toutes } & \multicolumn{3}{|c|}{ Essentiellement urbaines/Toutes } \\
\hline & 2004 & 2006 & 2007 & 2004 & 2006 & 2007 & 2004 & 2006 & 2007 \\
\hline Exploitations de la population & 0.2 & 0.2 & 0.2 & 0.7 & 0.7 & 0.7 & 0.1 & 0.1 & 0.1 \\
\hline Unité de travail (éq. temps plein) & 0.2 & 0.2 & 0.2 & 0.7 & 0.7 & 0.7 & 0.1 & 0.1 & 0.1 \\
\hline Superficie agricole utilisée & 0.3 & 0.3 & 0.3 & 0.6 & 0.6 & 0.6 & 0.1 & 0.1 & 0.1 \\
\hline Production agricole brute ${ }^{1}$ & 0.6 & 0.7 & 0.8 & 1.1 & 1.1 & 1.1 & 1.2 & 1.1 & 1.1 \\
\hline Soutien total & 0.8 & 0.9 & 0.8 & 1.1 & 1.1 & 1.1 & 1.1 & 0.8 & 1.0 \\
\hline Tous paiements ${ }^{2}$ & 0.9 & 1.0 & 0.9 & 1.0 & 1.1 & 1.1 & 1.0 & 0.7 & 0.8 \\
\hline Paiements du 1er pilier & 0.9 & 0.7 & 0.8 & 1.0 & 1.1 & 1.1 & 1.1 & 0.7 & 0.8 \\
\hline Paiements du 2ème pilier & 1.3 & 2.1 & 2.0 & 0.9 & 0.7 & 0.7 & 0.8 & 0.6 & 0.3 \\
\hline Autres paiements & 2.1 & 1.7 & 2.2 & 0.7 & 0.9 & 0.6 & 0.1 & 0.2 & 1.0 \\
\hline Soutien des prix du marché ${ }^{3}$ & 0.5 & 0.6 & 0.7 & 1.1 & 1.1 & 1.1 & 1.3 & 1.3 & 1.3 \\
\hline Recettes brutes ${ }^{4}$ & 0.7 & 0.7 & 0.8 & 1.1 & 1.1 & 1.1 & 1.2 & 1.0 & 1.1 \\
\hline Dépenses d'exploitation & 0.6 & 0.7 & 0.7 & 1.1 & 1.1 & 1.1 & 1.1 & 0.9 & 1.0 \\
\hline Revenu net d'exploitation 5 & 1.8 & 1.6 & 1.9 & 0.6 & 0.6 & 0.6 & 2.0 & 2.4 & 1.7 \\
\hline Amortissements ${ }^{6}$ & 0.7 & 0.8 & 0.8 & 1.1 & 1.1 & 1.1 & 1.2 & 1.0 & 1.0 \\
\hline Revenu agricole $^{7}$ & -1.2 & -1.2 & -8.4 & 1.9 & 2.2 & 5.3 & -0.1 & -2.3 & -5.5 \\
\hline Revenu non agricole $^{8}$ & n.a. & n.a. & n.a. & n.a. & n.a. & n.a. & n.a. & n.a. & n.a. \\
\hline Revenu des ménages agricoles ${ }^{9}$ & n.a. & n.a. & n.a. & n.a. & n.a. & n.a. & n.a. & n.a. & n.a. \\
\hline Total des actifs agricoles ${ }^{11}$ & n.a. & n.a. & n.a. & n.a. & n.a. & n.a. & n.a. & n.a. & n.a. \\
\hline Total des dettes agricoles ${ }^{12}$ & n.a. & n.a. & n.a. & n.a. & n.a. & n.a. & n.a. & n.a. & n.a. \\
\hline Capitaux propres agricoles ${ }^{13}$ & n.a. & n.a. & n.a. & n.a. & n.a. & n.a. & n.a. & n.a. & n.a. \\
\hline
\end{tabular}

Notes identiques à celles du tableau $\mathrm{C} 1$.

Source : tableaux transmis par les participants. 
Tableau annexe C8. Part du soutien dans les recettes des exploitations et dans le revenu agricole, par type de régions, certains pays de l'OCDE, 2004, 2006 et 2007

\begin{tabular}{|c|c|c|c|c|c|c|c|c|c|c|c|c|}
\hline \multirow{3}{*}{$\begin{array}{l}\text { Pays } \\
\text { Régions } \\
\text { Année }\end{array}$} & \multicolumn{12}{|c|}{ Canada } \\
\hline & \multicolumn{3}{|c|}{ Toutes exploitations } & \multicolumn{3}{|c|}{ Essentiellement rurales } & \multicolumn{3}{|c|}{ Intermédiaires } & \multicolumn{3}{|c|}{ Essentiellement urbaines } \\
\hline & 2004 & 2006 & 2007 & 2004 & 2006 & 2007 & 2004 & 2006 & 2007 & 2004 & 2006 & 2007 \\
\hline Part du soutien dans les recettes brutes ${ }^{1}$ & 15 & 17 & 13 & 16 & 17 & 14 & 15 & 17 & 15 & 10 & 11 & 9 \\
\hline Tous paiements ${ }^{2}$ & 9 & 9 & 7 & 9 & 9 & 8 & 8 & 8 & 7 & 5 & 5 & 4 \\
\hline Paiements du 1er pilier & n.a. & n.a. & n.a. & n.a. & n.a. & n.a. & n.a. & n.a. & n.a. & n.a. & n.a. & n.a. \\
\hline Paiements du 2ème pilier & n.a. & n.a. & n.a. & n.a. & n.a. & n.a. & n.a. & n.a. & n.a. & n.a. & n.a. & n.a. \\
\hline Autres paiements & 9 & 9 & 7 & 9 & 9 & 8 & 8 & 8 & 7 & 5 & 5 & 4 \\
\hline Soutien des prix du marché ${ }^{3}$ & 7 & 8 & 6 & 6 & 8 & 6 & 8 & 9 & 7 & 5 & 6 & 5 \\
\hline Part du soutien dans le revenu agricole & 280 & 384 & 211 & 261 & 384 & 200 & 319 & 335 & 249 & 676 & 579 & 297 \\
\hline Pays & \multicolumn{12}{|c|}{ États-Unis } \\
\hline Régions & \multicolumn{3}{|c|}{ Toutes exploitations } & \multicolumn{3}{|c|}{ Essentiellement rurales } & \multicolumn{3}{|c|}{ Intermédiaires } & \multicolumn{3}{|c|}{ Essentiellement urbaines } \\
\hline Année & 2004 & 2006 & 2007 & 2004 & 2006 & 2007 & 2004 & 2006 & 2007 & 2004 & 2006 & 2007 \\
\hline Part du soutien dans les recettes brutes ${ }^{1}$ & 9 & 6 & 7 & n.a. & 7 & 6 & n.a. & 8 & 8 & n.a. & 5 & 7 \\
\hline Tous paiements ${ }^{2}$ & 4 & 4 & 3 & n.a. & 6 & 4 & n.a. & 5 & 3 & n.a. & 3 & 2 \\
\hline Paiements du 1er pilier & 0 & 0 & 0 & n.a. & 0 & 0 & n.a. & 0 & 0 & n.a. & 0 & 0 \\
\hline Paiements du 2ème pilier & 0 & 0 & 0 & n.a. & 0 & 0 & n.a. & 0 & 0 & n.a. & 0 & 0 \\
\hline Autres paiements & 0 & 0 & 0 & n.a. & 0 & 0 & n.a. & 0 & 0 & n.a. & 0 & 0 \\
\hline Soutien des prix du marché ${ }^{3}$ & 4 & 2 & 4 & n.a. & 1 & 2 & n.a. & 2 & 4 & n.a. & 2 & 5 \\
\hline Part du soutien dans le revenu agricole & 39 & 37 & 33 & n.a. & 42 & 30 & n.a. & 48 & 42 & n.a. & 28 & 31 \\
\hline Pays & \multicolumn{12}{|c|}{ Angleterre } \\
\hline Régions & \multicolumn{3}{|c|}{ Toutes exploitations } & \multicolumn{3}{|c|}{ Essentiellement rurales } & \multicolumn{3}{|c|}{ Intermédiaires } & \multicolumn{3}{|c|}{ Essentiellement urbaines } \\
\hline Année & 2004 & 2006 & 2007 & 2004 & 2006 & 2007 & 2004 & 2006 & 2007 & 2004 & 2006 & 2007 \\
\hline Part du soutien dans les recettes brutes ${ }^{1}$ & 40 & 30 & 28 & n.a. & 32 & 29 & n.a. & 23 & 21 & n.a. & 18 & 17 \\
\hline Tous paiements $^{2}$ & 22 & 15 & 14 & n.a. & 16 & 14 & n.a. & 12 & 11 & n.a. & 8 & 8 \\
\hline Paiements du 1er pilier & 21 & 13 & 11 & n.a. & 13 & 11 & n.a. & 10 & 9 & n.a. & 7 & 6 \\
\hline Paiements du 2ème pilier & 2 & 3 & 3 & n.a. & 3 & 3 & n.a. & 2 & 2 & n.a. & 1 & 1 \\
\hline Autres paiements & 0 & 0 & 0 & n.a. & 0 & 0 & n.a. & 0 & 0 & n.a. & 0 & 0 \\
\hline Soutien des prix du marché ${ }^{3}$ & 17 & 15 & 14 & n.a. & 16 & 15 & n.a. & 11 & 10 & n.a. & 10 & 9 \\
\hline Part du soutien dans le revenu agricole & 252 & 173 & 129 & n.a. & 177 & 132 & n.a. & 145 & 106 & n.a. & 131 & 99 \\
\hline Pays & \multicolumn{12}{|c|}{ Allemagne } \\
\hline Régions & \multicolumn{3}{|c|}{ Toutes exploitations } & Esser & ellement & urales & & ermédiai & & Essent & llement & baines \\
\hline Année & 2004 & 2006 & 2007 & 2004 & 2006 & 2007 & 2004 & 2006 & 2007 & 2004 & 2006 & 2007 \\
\hline Part du soutien dans les recettes brutes ${ }^{1}$ & 27 & 23 & 20 & 23 & 20 & 18 & 27 & 23 & 20 & 32 & 28 & 24 \\
\hline Tous paiements ${ }^{2}$ & 14 & 14 & 14 & 12 & 11 & 12 & 14 & 14 & 14 & 16 & 17 & 17 \\
\hline Paiements du 1er pilier & 10 & 11 & 11 & 9 & 9 & 10 & 11 & 11 & 11 & 11 & 12 & 12 \\
\hline Paiements du 2ème pilier & 2 & 2 & 2 & 2 & 2 & 2 & 2 & 2 & 2 & 3 & 3 & 3 \\
\hline Autres paiements & 1 & 1 & 1 & 1 & 1 & 1 & 1 & 1 & 1 & 1 & 1 & 1 \\
\hline Soutien des prix du marché ${ }^{3}$ & 14 & 9 & 6 & 12 & 9 & 6 & 13 & 9 & 6 & 16 & 11 & 7 \\
\hline Part du soutien dans le revenu agricole & 207 & 149 & 121 & 170 & 127 & 106 & 208 & 152 & 121 & 246 & 167 & 135 \\
\hline Pays & & & & & & & & & & & & \\
\hline Régions & Toute & exploit & ions & Essen & ellement & urales & & rmédiai & & Essent & llement & baines \\
\hline Année & 2004 & 2006 & 2007 & 2004 & 2006 & 2007 & 2004 & 2006 & 2007 & 2004 & 2006 & 2007 \\
\hline Part du soutien dans les recettes brutes ${ }^{1}$ & 13 & 13 & 14 & 16 & 17 & 15 & 13 & 13 & 14 & 12 & 10 & 12 \\
\hline Tous paiements $^{2}$ & 10 & 12 & 9 & 14 & 15 & 11 & 9 & 11 & 9 & 8 & 8 & 7 \\
\hline Paiements du 1er pilier & 9 & 10 & 8 & 11 & 10 & 8 & 8 & 10 & 9 & 8 & 7 & 6 \\
\hline Paiements du 2ème pilier & 1 & 2 & 1 & 2 & 5 & 2 & 1 & 1 & 1 & 1 & 1 & 0 \\
\hline Autres paiements & 0 & 0 & 0 & 1 & 0 & 0 & 0 & 0 & 0 & 0 & 0 & 0 \\
\hline Soutien des prix du marché ${ }^{3}$ & 3 & 2 & 5 & 3 & 1 & 4 & 4 & 2 & 5 & 4 & 2 & 6 \\
\hline Part du soutien dans le revenu agricole & -322 & -408 & -1228 & 218 & 295 & 123 & -178 & -200 & -246 & 3161 & 133 & 215 \\
\hline
\end{tabular}


Tableau annexe C9. Indices de Gini dans certains pays de l'OCDE, par composante du revenu, 2004, 2006 et 2007

\begin{tabular}{|c|c|c|c|c|c|c|}
\hline & \multicolumn{3}{|c|}{ Canada } & \multicolumn{3}{|c|}{ États-Unis } \\
\hline & 2004 & 2006 & 2007 & 2004 & 2006 & 2007 \\
\hline \multicolumn{7}{|l|}{ Composantes du revenu } \\
\hline Production agricole brute ${ }^{1}$ & 0.58 & 0.60 & 0.60 & 0.64 & 0.63 & 0.64 \\
\hline Soutien total & 0.61 & 0.61 & 0.65 & 0.76 & 0.73 & 0.80 \\
\hline Tous paiements $^{2}$ & 0.62 & 0.61 & 0.67 & 0.73 & 0.73 & 0.73 \\
\hline Paiements du 1er pilier & n.a. & n.a. & n.a. & n.a. & n.a. & n.a. \\
\hline Paiements du 2ème pilier & n.a. & n.a. & n.a. & n.a. & n.a. & n.a. \\
\hline Autres paiements & 0.62 & 0.61 & 0.67 & n.a. & n.a. & n.a. \\
\hline Soutien des prix du marché ${ }^{3}$ & 0.48 & 0.48 & 0.48 & 0.96 & 0.98 & 0.98 \\
\hline \multirow[t]{3}{*}{ Recettes brutes ${ }^{4}$} & 0.56 & 0.58 & 0.58 & 0.65 & 0.63 & 0.63 \\
\hline & \multicolumn{3}{|c|}{ UE27 } & \multicolumn{3}{|c|}{ UE15 } \\
\hline & 2004 & 2006 & 2007 & 2004 & 2006 & 2007 \\
\hline \multicolumn{7}{|l|}{ Composantes du revenu } \\
\hline Production agricole brute ${ }^{1}$ & 0.70 & 0.71 & 0.73 & 0.67 & 0.69 & 0.69 \\
\hline Soutien total & 0.74 & 0.72 & 0.75 & 0.72 & 0.71 & 0.70 \\
\hline Tous paiements $^{2}$ & 0.73 & 0.71 & 0.75 & 0.70 & 0.70 & 0.70 \\
\hline Paiements du 1er pilier & 0.73 & 0.73 & 0.76 & 0.71 & 0.70 & 0.71 \\
\hline Paiements du 2ème pilier & 0.90 & 0.84 & 0.88 & 0.87 & 0.85 & 0.86 \\
\hline Autres paiements & n.a. & n.a. & n.a. & n.a. & n.a. & n.a. \\
\hline Soutien des prix du marché ${ }^{3}$ & 0.84 & 0.83 & 0.85 & 0.83 & 0.82 & 0.81 \\
\hline \multirow[t]{3}{*}{ Recettes brutes ${ }^{4}$} & 0.68 & 0.68 & 0.72 & 0.65 & 0.67 & 0.67 \\
\hline & \multicolumn{3}{|c|}{ UE10 } & \multicolumn{3}{|c|}{ UE2 } \\
\hline & 2004 & 2006 & 2007 & 2004 & 2006 & 2007 \\
\hline \multicolumn{7}{|l|}{ Composantes du revenu } \\
\hline Production agricole brute ${ }^{1}$ & 0.71 & 0.68 & 0.69 & n.a. & n.a. & 0.62 \\
\hline Soutien total & 0.74 & 0.68 & 0.69 & n.a. & n.a. & 0.75 \\
\hline Tous paiements $^{2}$ & 0.71 & 0.68 & 0.67 & n.a. & n.a. & 0.82 \\
\hline Paiements du 1er pilier & 0.69 & 0.70 & 0.70 & n.a. & n.a. & 0.82 \\
\hline Paiements du 2ème pilier & 0.97 & 0.78 & 0.77 & n.a. & n.a. & 1.00 \\
\hline Autres paiements & n.a. & n.a. & n.a. & n.a. & n.a. & n.a. \\
\hline Soutien des prix du marché ${ }^{3}$ & 0.82 & 0.79 & 0.83 & n.a. & n.a. & 0.72 \\
\hline Recettes brutes ${ }^{4}$ & 0.69 & 0.66 & 0.67 & n.a. & n.a. & 0.64 \\
\hline
\end{tabular}




\begin{tabular}{|c|c|c|c|c|c|c|}
\hline & \multicolumn{3}{|c|}{ Angleterre } & \multicolumn{3}{|c|}{ Estonie } \\
\hline & 2004 & 2006 & 2007 & 2004 & 2006 & 2007 \\
\hline \multicolumn{7}{|l|}{ Composantes du revenu } \\
\hline Production agricole brute ${ }^{1}$ & 0.61 & 0.62 & 0.62 & 0.77 & 0.77 & 0.77 \\
\hline Soutien total & 0.53 & 0.54 & 0.55 & 0.73 & 0.67 & 0.64 \\
\hline Tous paiements ${ }^{2}$ & 0.59 & 0.55 & 0.54 & 0.73 & 0.65 & 0.66 \\
\hline Paiements du 1er pilier & 0.60 & 0.55 & 0.54 & 0.69 & 0.70 & 0.71 \\
\hline Paiements du 2ème pilier & 0.82 & 0.72 & 0.70 & 0.80 & 0.66 & 0.65 \\
\hline Autres paiements & 0.91 & 0.98 & 0.98 & 0.96 & 0.90 & 0.99 \\
\hline Soutien des prix du marché ${ }^{3}$ & 0.66 & 0.69 & 0.72 & 0.83 & 0.81 & 0.79 \\
\hline \multirow[t]{3}{*}{ Recettes brutes ${ }^{4}$} & 0.55 & 0.58 & 0.58 & 0.74 & 0.73 & 0.74 \\
\hline & \multicolumn{3}{|c|}{ Flandre belge } & \multicolumn{3}{|c|}{ Pays-Bas } \\
\hline & 2004 & 2006 & 2007 & 2004 & 2006 & 2007 \\
\hline \multicolumn{7}{|l|}{ Composantes du revenu } \\
\hline Production agricole brute ${ }^{1}$ & 0.45 & 0.47 & 0.46 & 0.51 & 0.55 & 0.54 \\
\hline Soutien total & 0.48 & 0.48 & 0.50 & 0.61 & 0.59 & 0.66 \\
\hline Tous paiements $^{2}$ & 0.50 & 0.50 & 0.50 & 0.71 & 0.70 & 0.70 \\
\hline Paiements du 1er pilier & 0.55 & 0.54 & 0.53 & 0.73 & 0.70 & 0.70 \\
\hline Paiements du 2ème pilier & 0.72 & 0.69 & 0.73 & 0.95 & 0.94 & 0.94 \\
\hline Autres paiements & 0.98 & 0.78 & 0.73 & 0.94 & 0.97 & 0.96 \\
\hline Soutien des prix du marché ${ }^{3}$ & 0.53 & 0.54 & 0.60 & 0.64 & 0.62 & 0.73 \\
\hline \multirow[t]{3}{*}{ Recettes brutes ${ }^{4}$} & 0.43 & 0.45 & 0.44 & 0.49 & 0.53 & 0.53 \\
\hline & \multicolumn{3}{|c|}{ France } & \multicolumn{3}{|c|}{ Allemagne } \\
\hline & 2004 & 2006 & 2007 & 2004 & 2006 & 2007 \\
\hline \multicolumn{7}{|l|}{ Composantes du revenu } \\
\hline Production agricole brute ${ }^{1}$ & 0.49 & 0.50 & 0.51 & 0.56 & 0.55 & 0.55 \\
\hline Soutien total & n.a. & n.a. & n.a. & 0.57 & 0.56 & 0.56 \\
\hline Tous paiements $^{2}$ & 0.50 & 0.49 & 0.50 & 0.60 & 0.58 & 0.56 \\
\hline Paiements du 1er pilier & 0.55 & 0.54 & 0.54 & 0.63 & 0.60 & 0.58 \\
\hline Paiements du 2ème pilier & 0.84 & 0.84 & 0.86 & 0.63 & 0.62 & 0.63 \\
\hline Autres paiements & 0.76 & 0.78 & 0.83 & 0.65 & 0.57 & 0.55 \\
\hline Soutien des prix du marché ${ }^{3}$ & n.a. & n.a. & n.a. & 0.62 & 0.60 & 0.60 \\
\hline Recettes brutes ${ }^{4}$ & 0.45 & 0.46 & 0.47 & 0.54 & 0.54 & 0.54 \\
\hline
\end{tabular}

1. Inclut les recettes tirées des ventes de produits et de services agricoles tels que le travail à façon ou la mise en location de terres, de quotas, de bâtiments ou de machines, entre autres.

2. Inclut les transferts budgétaires aux agriculteurs résultant de la politique agricole et des paiements des systèmes d'assurance, des programmes de conservation des terres aux États-Unis et des premier et second piliers de la Politique agricole commune dans l'Union européenne.

3. Le soutien des prix du marché (SPM) est calculé en appliquant le ratio SPM de chaque produit de base, lorsqu'il existe dans la base de données des ESP de l'OCDE, aux recettes de l'exploitation tirées de ce produit. Le ratio SPM moyen de l'ensemble des produits de la base ESP est utilisé pour les recettes provenant de produits pour lesquels il n'existe pas de ratio SPM.

4. Comprend la production agricole brute et l'ensemble des paiements.

Source : tableaux transmis par les participants. 